\title{
Identifying the elephant
}

Citation for published version (APA):

Bowman, A. (2021). Identifying the elephant: technological developments towards the complete identification of lipids. [Doctoral Thesis, Maastricht University]. Maastricht University. https://doi.org/10.26481/dis.20210831ab

Document status and date:

Published: 01/01/2021

DOI:

10.26481/dis.20210831ab

Document Version:

Publisher's PDF, also known as Version of record

\section{Please check the document version of this publication:}

- A submitted manuscript is the version of the article upon submission and before peer-review. There can be important differences between the submitted version and the official published version of record.

People interested in the research are advised to contact the author for the final version of the publication, or visit the DOI to the publisher's website.

- The final author version and the galley proof are versions of the publication after peer review.

- The final published version features the final layout of the paper including the volume, issue and page numbers.

Link to publication

\footnotetext{
General rights rights.

- You may freely distribute the URL identifying the publication in the public portal. please follow below link for the End User Agreement:

www.umlib.nl/taverne-license

Take down policy

If you believe that this document breaches copyright please contact us at:

repository@maastrichtuniversity.nl

providing details and we will investigate your claim.
}

Copyright and moral rights for the publications made accessible in the public portal are retained by the authors and/or other copyright owners and it is a condition of accessing publications that users recognise and abide by the legal requirements associated with these

- Users may download and print one copy of any publication from the public portal for the purpose of private study or research.

- You may not further distribute the material or use it for any profit-making activity or commercial gain

If the publication is distributed under the terms of Article $25 \mathrm{fa}$ of the Dutch Copyright Act, indicated by the "Taverne" license above, 
IDENTIFYING THE ELEPHANT:

TECHNOLOGICAL

DEVELOPMENTS TOWARDS

THE COMPLETE

IDENTIFICATION OF LIPIDS

Andrew Bowman 
(C)2021 Maastricht University_Faculty of Health, Medicine \& Life Sciences

Self-published, Andrew Bowman, Maastricht (The Netherlands)

Cover design-

Print-

Alle rechten voorbehouden. Niets uit deze uitgave mag worden vermenigvuldigd en/of openbaar gemaakt worden door middel van druk, fotokopie, microfilm, elektronisch of op welke andere wijze ook zonder voorafgaande schriftelijke toestemming van de uitgever.

All rights reserved. No part of this publication may be reproduced in any form by print, photoprint, microfilm, electronic or any other means without written permission from the publisher.

ISBN - 


\section{IDENTIFYING THE ELEPHANT: TECHNOLOGICAL \\ DEVELOPMENTS TOWARDS THE COMPLETE IDENTIFICATION OF LIPIDS}

\section{Dissertation}

To obtain the degree of Doctor at Maastricht University,

On the authority of the Rector Magnificus

$$
\text { Prof.dr. Rianne M. Letschert, }
$$

In accordance with the decision of the Board of Deans,

$$
\text { To be defended in public on }
$$

Tuesday, 31st of August 2021 at 10:00

By

\section{Andrew Bowman}

Born in Wichita, Kansas, USA, on December 9, 1990 


\section{Supervisor:}

Prof. Dr. Ron M.A. Heeren

\section{Co-Supervisor:}

Prof. Dr. Shane Ellis

\section{Assessment Committee:}

Prof. Dr. J. Plat (Chair), Maastricht University

Prof. Dr. E. Biessen, Maastricht University

Prof Dr. E. Blaak, Maastricht University

Prof. Dr. G. Reid, University of Melbourne

Prof. Dr. E. de Pauw, University of Liège

The research in this thesis has been made possible with funding from the "Interreg Euro-Maas-Rijn” project (project number EMR23). 


\section{TABLE OF CONTENTS}

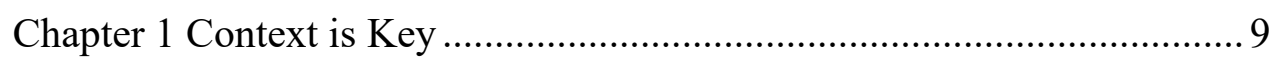

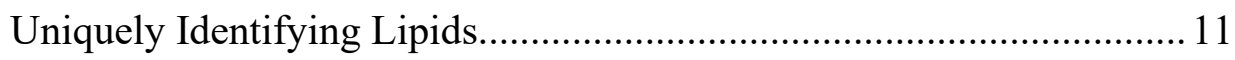

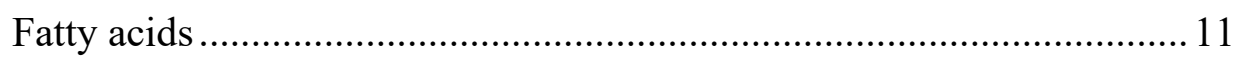

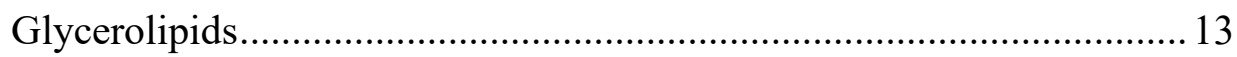

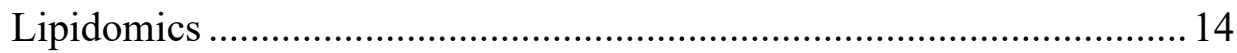

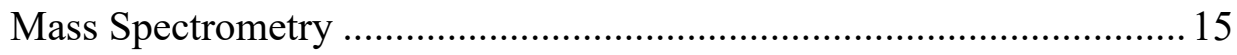

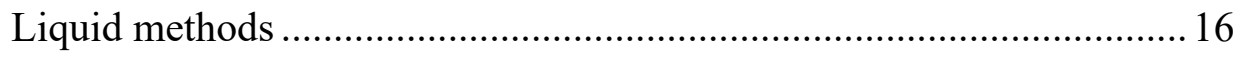

Mass Spectrometry Imaging ....................................................... 18

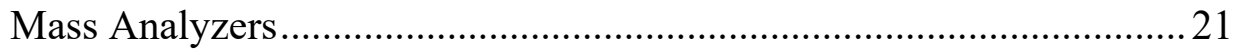

Hybrid Mass Spectrometers and Tandem Mass Spectrometry ............... 23

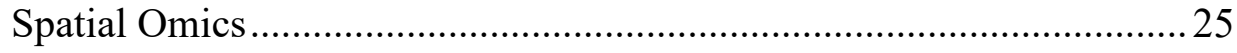

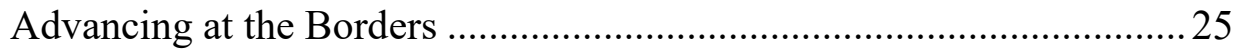

Chapter 2 Advances in Mass Spectrometry Imaging Enabling Visualization and Observation of Localized Lipid Biochemistry within Tissues ............ 27

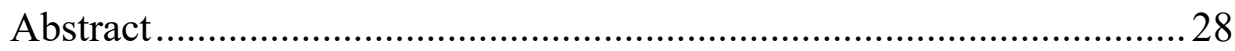

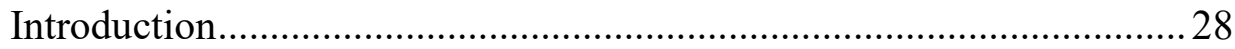

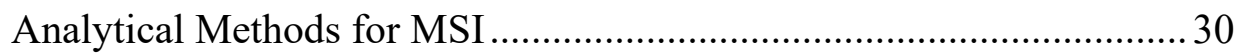

Applications of MSI to localized lipid biochemistry ............................. 31

Adding temporal data: Visualizing local lipid dynamics in tissues........ 38

Current analytical challenges and future opportunities in lipid MSI...... 41

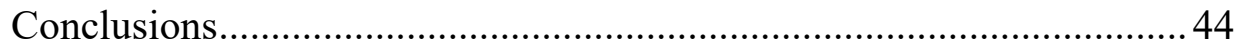

Chapter 3 Ultra-High Mass Resolving Power, Mass Accuracy, and Dynamic Range MALDI Mass Spectrometry Imaging by 21-Tesla FT-ICR MS..... 47

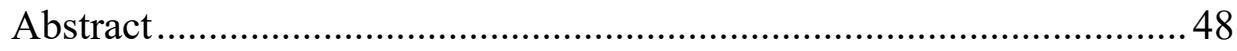

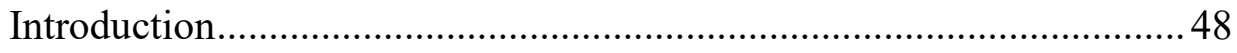


Methods 52

Results and Discussion 54

Conclusion

Chapter 4 Evaluation of Lipid Coverage and High Spatial Resolution MALDI-Imaging Capabilities of Oversampling Combined with Laser PostIonization 73

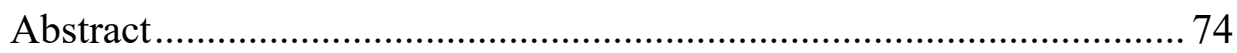

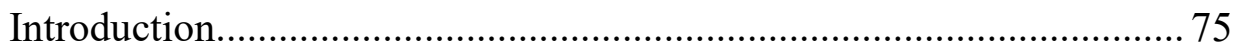

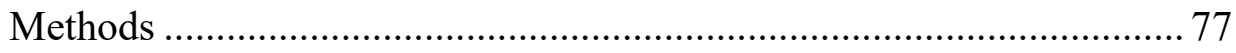

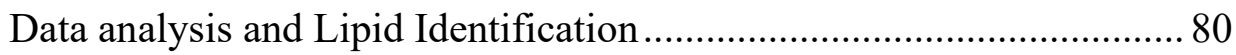

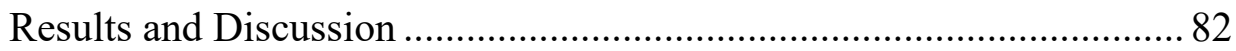

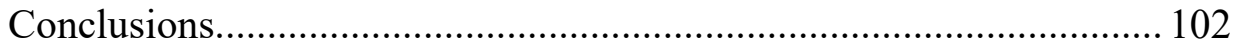

Chapter 5 High Throughput and High Sensitivity Isomer-resolved Imaging of Lipids by Integration of Ozone-induced dissociation with a MALDI-

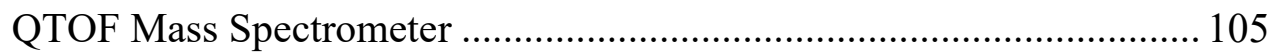

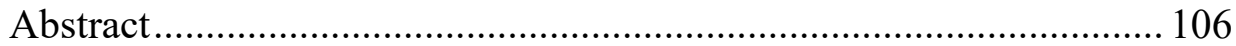

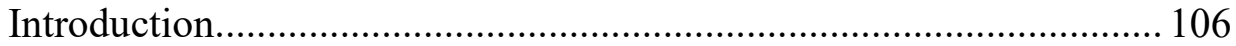

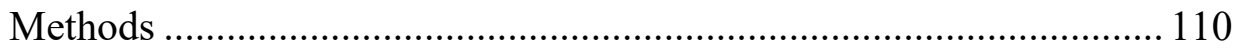

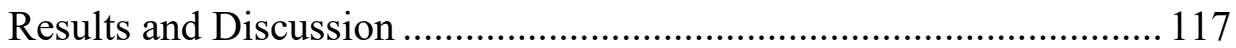

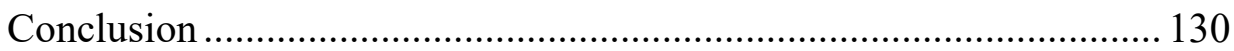

Chapter 6 Negative Mode Imaging of Phospholipids Using Ozone-Induced Dissociation.................................................................................... 131

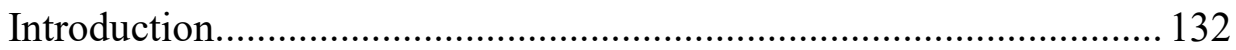

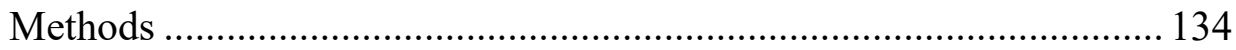

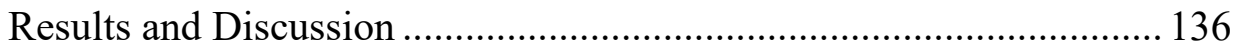

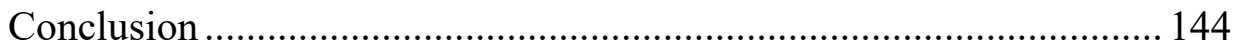

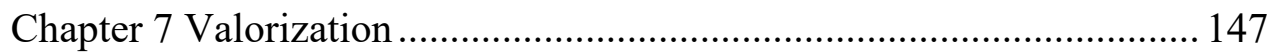

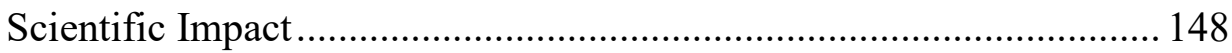




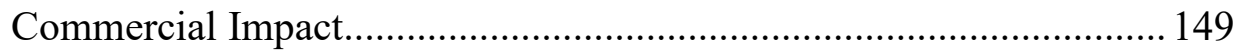

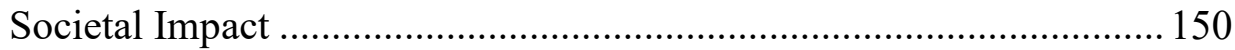

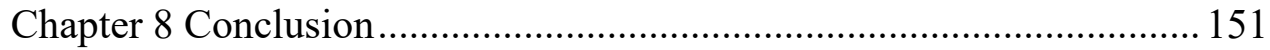

Biological Context in Mass Spectrometry Imaging............................. 152

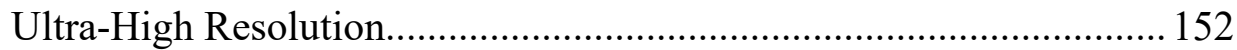

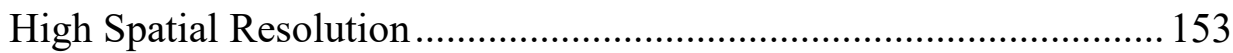

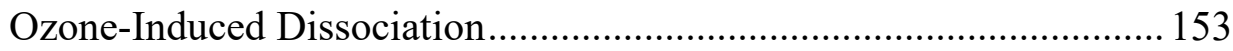

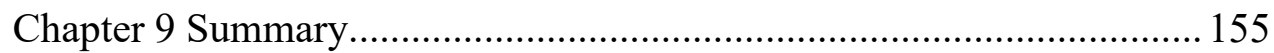

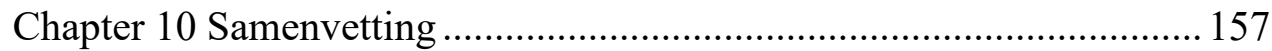

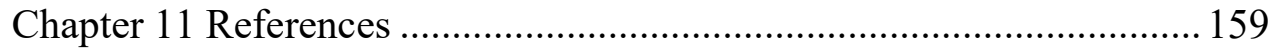

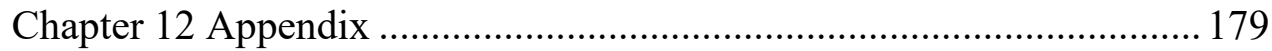

Funding And Conflicts of Interest ................................................... 180

Chapter 13 List of Publications............................................................ 181

Chapter 14 Acknowledgements ............................................................ 185

Chapter 15 Curriculum Vitae ................................................................. 189 



\section{Chapter 1 CONTEXT IS KEY}


There is an ancient Indian proverb about 6 blind men encountering an elephant for the first time. One of the men grabs hold of the elephant's tusk and declares that an elephant is like a spear. The second grabs its leg, stating that it is instead like a tree. A third runs his hand along its ear, saying that an elephant must be a fan. The fourth touches its side, sure that an elephant is a wall. The fifth finds his hand wrapped in the elephant's trunk and exclaims that an elephant is a snake. The last touches the hairy tip of the elephant's tail and says that an elephant is simply a type of mouse. Each piece of evidence makes sense in isolation, but none of them truly reflect the whole. Only in combining these disparate ideas can you start to make an accurate representation of an elephant.

The progress of science is much the same. Blind men, groping in the dark to gather a piece of information that we hope we can make sense of. Single experiments often lead to wrong interpretations, but, with the weight of time and more experiments, we build up a better understanding of reality. Take 2 of the conclusions about the elephant, the ears (a fan) and the leg (a tree). Combining these two it is possible to conclude that perhaps the ear is not a fan, but instead a particularly large leaf. Another wrong answer, but with a further kernel of truth: it is one whole, and it's probably not a pine tree. Eventually, if you take all 6 blind men, we can begin to reconstruct a rough approximation of an elephant. A heavy body supported by several pillars, with a pair leathery fans at one end, with a muscular tube and a pair of spears beneath, and a thin rope with a tuft of fur at the far side. If we brought in more blind men, who each touched yet another part of the elephant, we could get ever closer to describing an elephant. Alternatively, if we could improve the amount of the elephant any individual could touch or refine their senses for more precise information, we would also be able to build up a more complete view of the elephant.

The crux of my doctoral work has been in trying to figure out how to better examine the elephant that is the lipidome. Unfortunately, the metaphor is going to break down a bit here, as the elephant in question is more akin to one made of smaller elephants. The study of the greater lipidome is highly dependent on the ability to examine any individual lipid. Lipids can be examined through mass spectrometry, though simple, one stage experiments are the equivalent of being able to distinguish an elephant from a rhinoceros. Helpful, if you are looking for any kind of elephant, less helpful if you are attempting to distinguish an African from an Asian elephant, or a male from a female elephant. Describing the lipidome is 
much more complex, and necessitates either more complex experimental design, or multiple time-and-space delineated experiments. Before we get deeply into the mass spectral end of my thesis, let us start by defining the lipid elephant.

\section{UNIQUELY IDENTIFYING LIPIDS}

Lipids are defined rather broadly as any biomolecule that is soluble in nonpolar solvents[1]. This covers fatty acids, sterols, prenols, saccharolipids, polyketides, sphingolipids, and the glycerides (mono-, di-, and tri-, as well as phospholipids). Of these, the fatty acids and the fatty acid derivatives will be our first focus. Fatty acids serve as a basic carbon and energy storage medium, but typically are incorporated into larger, more complex macrobiomolecules[2, 3], including the glycerides and sphingolipids. Fatty acids are bound to sphingolipids via amide bonds[4], and to glycerides via esterification (usually)[5]. Both glycerides and sphingolipids are studied for their roles in cellular membranes[6], apoptosis[7], proliferation, stress response[8], necrosis[9], inflammation[10], autophagy[11], senescence[12], differentiation, inter- and intracellular signaling[13-15], energy storage[16], and metabolism[17]. Further, they are implicated in numerous[18] cardiovascular[19] disorders[20, 21], cancers[22-24], diabetes[25-27], Alzheimer's[28-30], and a[31] host[32] of[33] other[34] diseases[35] and[36] disorders[37, 38]. Due to their ubiquity and relative ease of ionization, they have become one of the foremost markers for investigating health states.

\section{FATTY ACIDS}

As biomolecules, lipids function as repositories of energy and carbon. While consisting of only two major pieces, an aliphatic chain and a carboxylic acid, they have enough complexity to be identified and named at multiple levels. Naming of fatty acids in mass spectrometry follows the generally agreed upon LIPID MAPS notation: the species level, DB-position level, structure defined level, full structure level, and the complete structure level (the "common name")[39]. In the fatty acids, the variable portion is the aliphatic chain, as the carboxylic acid is synonymous with the biomolecular class. 

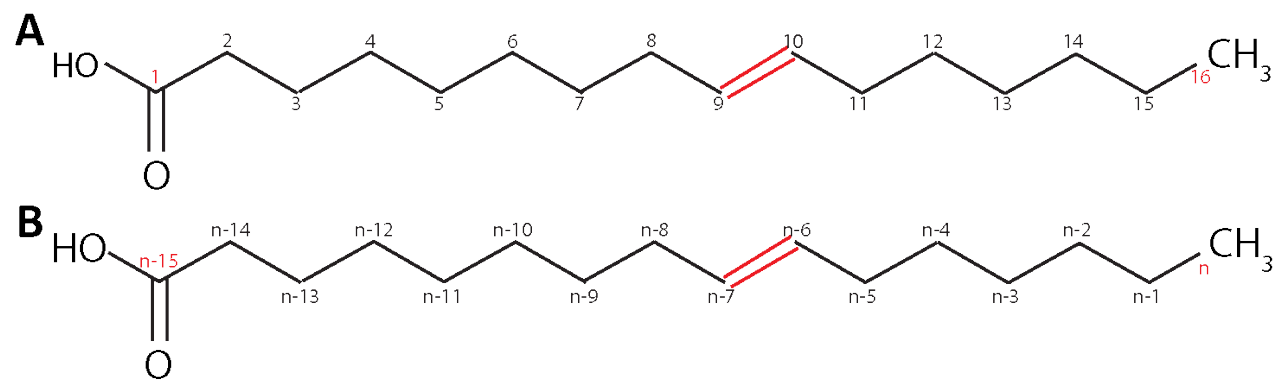

Hierarchical Level

$$
\text { Species }
$$

DB-Position

Full Structure

\section{Annotation}

FA 18:1

FA 18:1(9)

FA $16: 1(9 E)$

\section{Complete Structure $\longrightarrow$ Trans-Palmitoleate}

Figure 1.1. Labeling of fatty acid carbons according to $\Delta$ numbering (A), with acyl end n- numbering below (B). Identification of this fatty acid proceeds from least to most well-described: species, double bond position, full structure, complete structure.

Fatty acid chains vary based on the number of carbons (typically between 12 and 34, though as little as 1 is possible, the number of carbon-carbon double bonds (and their geometry), the presence or absence of cyclic groups, branches, or hydroxyl groups. All told, there are potentially millions of possible fatty acids. An example fatty acid is provided in Figure 1.1. This is a simple fatty acid, containing no extra functional groups and only a single double bond and 18 carbons in its chain. Under LIPID MAPS, this could be identified as FA 18:1 at the species level. As there are no functional groups, the structure defined level is the same as the species level. By being able to define the double bond is at the ninth position (counting from the aliphatic end as in $\omega$ nomenclature), we can move to the DB-position level, FA 18:1(9). Identifying that the double bond in questions is cis in orientation, then the full structure level is FA 18:1(9Z). With this information, we can define this fatty acid as palmitoleic acid. Fatty acids like palmitoleic acid are among the most common types, but branching, cyclic, or functionalized aliphatic chains are all possible. The difficulty in identifying the more specific levels increases drastically with mass spectrometry, which I shall cover slightly later. Within the context of my 
work, the fatty acids are not studied on their own, but as part of the more complicated glycerolipids (GLs).

\section{GLYCEROLIPIDS}

GLs are formed by the attachment of fatty acids to a simple carbon and alcohol backbone: glycerol. The attachment is through the carboxylic acid, typically as an ester, though ether-linkages are also possible. Between 1 and 3 fatty acids can be bound to a single glycerol, producing fat storing mono-, di-, and triglycerides. The glycerides are associated with any number of diseases, including atherosclerosis, heart disease, and stroke, making identification of specific glycerides central to understanding the most common cardiovascular diseases. Identification of the glycerides is more complicated compared to the FAs, not just in terms of them containing 1 to $3 \mathrm{FAs}$, but because the glycerides always have chirality. This chirality is defined by the stereonumbering (sn-) of the FAs on the glycerol backbone, in essence meaning that a triglyceride with the makeup 1palmito-2-oleo-3-stearoyl is not the same as 1-stearo-2-oleo-3-palmitoyl. With FA chain length potentially going from a single carbon to more than 30 carbons, potential for several double bonds per FA, and their sn-alterations, the sheer magnitude of identifying specific lipids becomes ever more apparent.

As a subset of the GLs, the glycerophospholipids (GPLs) replace one of the 3 FAs with a phosphatidic acid, or a phosphate derivative. These GPLs are the most abundant lipids in most organisms, owing to their use as the bulk of cellular membranes. The main phospholipids are phosphatidic acids (PA), phosphatidylethanolamines (PE), phosphatidylcholines (PC), phosphatidylserines (PS), and the phosphatidylinositides (PI). Additionally, we can include two of the phosphosphingolipids in our count, though these a sphingosine backbone rather than glycerol: ceramides and sphingomyelins.

Defining GPLs similarly to the FAs, PC 34:1 would be an example of a species level name (Figure 1.2). More well-defined is the molecular species level, which also defines the specific fatty acyls on the phospholipid, transforming PC(34:1) into PC(16:0_18:1), with the underscore showing that the stereonumbering of the fatty acyls is still unknown. Clearly then, next would be the stereonumber-position level, in the example above defining the phospholipid as PC(16:0/18:1). If we could then identify the position of the double bond on the second fatty acyl, we could be at the DB-position level, identifying our lipid as PC 
16:0/18:1(9). The full structure level would define the DB geometry $(\operatorname{PC}(16: 0 / 18: 1(9 z)))$, and the complete structure level would define the stereochemistry (PC(R-16:0/18:1(9z))).

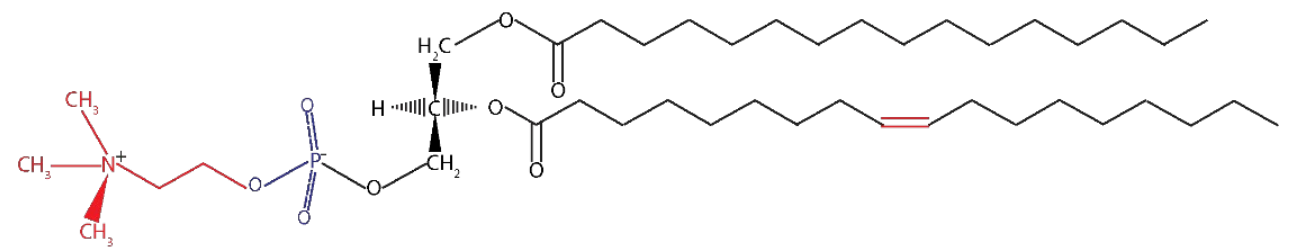

Hierarchical Level Annotation

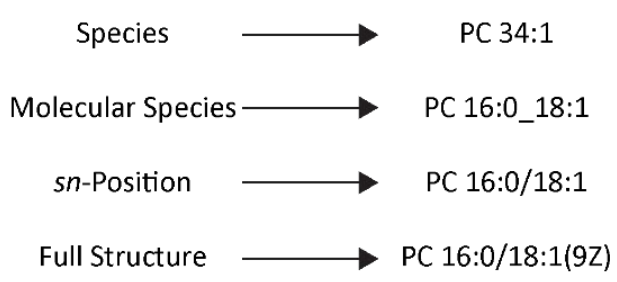

Figure 1.2. Hierarchical identification of a simple phosphatidylcholine with a single double bond.

\section{LIPIDOMICS}

At this point, the task of identifying any specific lipid amongst the nigh limitless potential lipids might seem like an impossible task. However, there are several factors help reduce this complexity. First, complex multicellular life produces only even numbers of carbons in lipids. Second, as far as has been determined, the phosphate headgroup in GPLs is only ever attached at the $s n-3$ position. Third, while trans fatty acids do exist in complex lifeforms, they are produced only by bacteria (such as found in the guts of ruminants). Further, even of those lipids left, it has been determined that only about 180,000 lipids comprise the lipidome[40], with many either very low in abundance or currently unobserved[41]. With such a significantly reduced list it is, theoretically, much simpler to identify lipids uniquely. It is important to note that lipid biochemistry changes with regard to tissue type, but also according to cell type within tissues. Truly analyzing the lipidome requires examining lipids while keeping their localization, a factor that will be discussed in the mass spectrometry section. 


\section{MASS SPECTROMETRY}

My tool of choice for identifying lipids and exploring the lipidome is mass spectrometry. At its simplest, mass spectrometry works by ionizing a molecule of interest and then separating ions and measuring their mass-to-charge ratio[42]. Ion sources are the most variable components, incorporating dozens if not hundreds of different methodologies. Mass analyzers used in lipidomics are also diverse, with some 5 common analyzers technologies spread across tens of different implementations. Most mass analyzers are part and parcel with a specific type of detector and will not be discussed in detail here. I will address a small fraction of the legion of ion sources available, and the 7 ionization methods (Table 1).

Of these, electron ejection and capture are "hard" ionization methods, meaning they create significant ion fragmentation in the process of generating ions. The other ionization methods are "soft," typically resulting in little to no fragmentation of the molecular ions, but this can be ion source dependent. A solid introduction to the ion sources often used to detect biomolecules can be found in The Expanding Role of Mass Spectrometry in Biotechnology[43], but I will focus on the 2 most common sources for liquid and imaging methodologies: Electrospray Ionization (ESI) and Matrix-Assisted Laser Desorption/Ionization (MALDI). These two techniques and their derivatives are preferred for their versatility, tunability, and the relative softness of their ionization. 


\begin{tabular}{|c|c|c|}
\hline Ionization Method & Advantage & Disadvantage \\
\hline Protonation (positive) & $\begin{array}{c}\text { Broadly applicable } \\
\text { Many ion sources generate these }\end{array}$ & $\begin{array}{l}\text { Compounds can be } \\
\text { unstable or do not readily } \\
\text { accept protons }\end{array}$ \\
\hline $\begin{array}{l}\text { Cationization } \\
\text { (positive) }\end{array}$ & $\begin{array}{c}\text { Broadly applicable } \\
\text { Tunable by cation } \\
\text { Many ion sources can generate } \\
\text { these }\end{array}$ & $\begin{array}{l}\text { Tandem mass spectrometry } \\
\text { experiments have limited } \\
\text { fragmentation information }\end{array}$ \\
\hline $\begin{array}{l}\text { Deprotonation } \\
\text { (negative) }\end{array}$ & $\begin{array}{l}\text { Useful for acidic compounds } \\
\text { Many ion sources generate these }\end{array}$ & $\begin{array}{l}\text { Highly compound } \\
\text { dependent }\end{array}$ \\
\hline $\begin{array}{l}\text { Anionization } \\
\text { (negative) }\end{array}$ & Tunable by anion & $\begin{array}{l}\text { Many ions are unstable or } \\
\text { metastable }\end{array}$ \\
\hline $\begin{array}{l}\text { Pre-charged molecule } \\
\text { transfer to gas phase } \\
\text { (both positive and } \\
\text { negative) }\end{array}$ & $\begin{array}{l}\text { Useful for pre-charged } \\
\text { molecules } \\
\text { Many ion sources generate these }\end{array}$ & $\begin{array}{l}\text { Only useful with pre- } \\
\text { charged ions }\end{array}$ \\
\hline $\begin{array}{l}\text { Electron ejection } \\
\text { (positive) }\end{array}$ & $\begin{array}{c}\text { Works with electron ionization } \\
\text { Provides fragmentation } \\
\text { information }\end{array}$ & $\begin{array}{l}\text { High rates of in-source } \\
\text { fragmentation } \\
\text { Molecular ion can be } \\
\text { difficult to determine }\end{array}$ \\
\hline $\begin{array}{l}\text { Electron capture } \\
\text { (negative) }\end{array}$ & $\begin{array}{l}\text { Works with electron ionization } \\
\text { Provides fragmentation } \\
\text { information }\end{array}$ & $\begin{array}{l}\text { High rates of in-source } \\
\text { fragmentation } \\
\text { Molecular ion can be } \\
\text { difficult to determine }\end{array}$ \\
\hline
\end{tabular}

Table 1-1. Ionization methods with general advantages and disadvantages of each.

\section{LIQUID METHODS}

ESI is perhaps the most widely used liquid method in mass spectrometry, seeing extensive use in research [44, 45], the pharmaceutical industry[46, 47], and the healthcare sector $[48,49]$. Ions are generated by running a voltage through a thin needle that the crude extract is pumped into, with the resulting charged particles desorbing and passing into the mass spectrometer. This electrospray ionization (ESI) can be done with either positive or negative charge, ionizing different biomolecules based on their ability to accept an ion (such as a positively charged proton or negatively charged chloride). More, it is easily adjusted to examine analytes in both positive and negative mode with little alteration, based on the constitution of the solution the analytes are dissolved in. The only limitation in ESI is whether a particular analyte can be extracted into a liquid medium, which also forms the major source of ion suppression, the matrix effect[50]. In its most basic sense, the matrix effect alters the signal response of analytes, either suppressing or enhancing them, thus distorting efforts towards quantitation and reproducibility. 
ESI, like all liquid methods, involves extracting lipids from the greater mass of the tissue, typically by grinding the solid mass and performing one of several extraction techniques, based on the non-polar solubility of the lipids. Common examples include the Folch[51] and Bligh-Dyer[52] methods, though as in all things mass spectrometry there are a wide variety of modifications and alternatives[53-57]. Nevertheless, the resulting lipid-containing liquid is then ionized and analyzed using a mass spectrometer. If the crude extract lipids are not separated from one another first, the method is a so-called "shotgun" method[58].

Alternatively, the crude extract can be partitioned before or after ionization. ESI is easily coupled to pre-ionization analyte separation, vis a vis liquid chromatography (LC)[59-61], or to post-ionization separation, including drift tube ion mobility (DTIMS)[62], travelling wave IMS (TWIMS)[63], differential IMS (DIMS)[64], or trapped IMS (TIMS)[65]. Separation beforehand is more common, as the methods to optimize separation of specific lipids are more robust, though both pre- and post-ionization separation can be combined in a single system[66, 67]. LC methods can be adapted based on the polarity of both the liquid and solid phases, and by the functionalization of the solid phase[68-75]. Depending on factors such as headgroup and fatty acid composition, lipids temporarily bind with a solid surface for differing lengths of times, separating them out chronologically and simplifying the mass spectra. Ion mobility methods, by contrast, sample the average cross section of the ions as they tumble in a gas, separating them based on their size.

Liquid methods are highly sensitive, owing to their ability to separate and concentrate the lipids in a complex biological sample. Further, identification of many different lipids is possible with targeted techniques, potentially identifying lipids from sum-composition down to the full structure level. There are, however, two flaws in this form of analysis for the elephantine lipidome. First, LC methods are selective, with techniques that work well for neutral lipids either removing or suppressing polar lipids[68-71], and vice versa[72-75]. Second, in the analogy of identifying the elephant, classical chromatographic experiments are akin to carving up the elephant and then identifying the different bits from one another. It is possible to identify tusks and the tail in a single experiment, but it would be impossible to say where the tusks go on the original elephant. Keeping the elephant more intact is the domain of mass spectrometry imaging. 


\section{MASS SPECTROMETRY IMAGING}

Rather than grinding and extracting lipids, imaging typically relies on making thin tissue sections and examining micrometer-sized pieces at a time. Creating ions from a single pixel is a rapidly evolving field, and new technologies emerge every year[76-94]. A non-exhaustive list of techniques is included in Figure 1.3 , including the name, type of ionization, commercially available resolution, and the highest reported resolution in each. Many of these techniques are liquid extraction-based, a la ESI, but extract from a small portion of a prepared surface, rather than from a ground and homogenized sample. The other techniques ablate material from the surface without bulk dissolution. 


\begin{tabular}{|c|c|c|c|}
\hline Ionization Technique & Type of Ionization & $\begin{array}{l}\text { Commercial } \\
\text { Imaging } \\
\text { Resolution }\end{array}$ & $\begin{array}{l}\text { Highest } \\
\text { Reported } \\
\text { Resolution }\end{array}$ \\
\hline $\begin{array}{l}\text { Desorption Electrospray } \\
\text { Ionization (DESI)[93] }\end{array}$ & Liquid Extraction & $100-200 \mu \mathrm{m}$ & $35 \mu \mathrm{m}[84]$ \\
\hline $\begin{array}{l}\text { Nano-Electrospray Desorption } \\
\text { Ionization (nano-DESI)[92] }\end{array}$ & Liquid Extraction & $50-100 \mu \mathrm{m}$ & $<10 \mu \mathrm{m}[76]$ \\
\hline $\begin{array}{l}\text { Air Flow Assisted Desorption } \\
\text { Electrospray Ionization } \\
\text { (AFADESI)[88] }\end{array}$ & Liquid Extraction & $200-300 \mu \mathrm{m}$ & $200 \mu \mathrm{m}$ \\
\hline $\begin{array}{l}\text { Easy Ambient Sonic-Spray } \\
\text { Ionization (EASI)[85] }\end{array}$ & Liquid Extraction & $100-200 \mu \mathrm{m}$ & $50 \mu \mathrm{m}$ \\
\hline $\begin{array}{l}\text { Laser Ablation Electrospray } \\
\text { Ionization (LAESI)[89] }\end{array}$ & $\begin{array}{l}\text { Two Step (Laser } \\
\text { ablation and ESI) }\end{array}$ & $200-300 \mu \mathrm{m}$ & $30 \mu \mathrm{m}$ \\
\hline Single Probe (SP)[91] & Liquid Extraction & $10-20 \mu \mathrm{m}$ & $8.5 \mu \mathrm{m}$ \\
\hline $\begin{array}{l}\text { Liquid Microjunction-Surface } \\
\text { Sampling Probe (LMJ-SSP)[94] }\end{array}$ & Liquid Extraction & $0.5-1 \mathrm{~mm}$ & $\begin{array}{c}<500 \\
\mu \mathrm{m}[77]\end{array}$ \\
\hline $\begin{array}{l}\text { Contact Mode-Scanning Probe } \\
\text { Electrospray Ionization/Tapping } \\
\text { Mode-Scanning Probe } \\
\text { Electrospray Ionization (c- } \\
\text { SPESI/t-SPESI)[90] }\end{array}$ & Liquid Extraction & $35-200 \mu \mathrm{m}$ & $35 \mu \mathrm{m}[87]$ \\
\hline $\begin{array}{l}\text { Laser Ablation Direct Analysis in } \\
\text { Real Time Imaging (LADI)[78] }\end{array}$ & $\begin{array}{l}\text { Two Step (laser } \\
\text { ablation and } \\
\text { plasma ionization) }\end{array}$ & $50 \mu \mathrm{m}$ & $50 \mu \mathrm{m}$ \\
\hline $\begin{array}{l}\text { Picosecond Infrared-Laser } \\
\text { Ablation Electrospray Ionization } \\
\text { (PIR-LAESI)[79] }\end{array}$ & $\begin{array}{l}\text { Two Step (laser } \\
\text { ablation and } \\
\text { electrospray } \\
\text { ionization) }\end{array}$ & $100 \mu \mathrm{m}$ & $100 \mu \mathrm{m}$ \\
\hline $\begin{array}{l}\text { Liquid Extraction Surface analysis } \\
\text { (LESA)[81] }\end{array}$ & Liquid Extraction & $1 \mathrm{~mm}$ & $300 \mu \mathrm{m}$ \\
\hline $\begin{array}{c}\text { Matrix-Assisted Laser } \\
\text { Desorption/Ionization } \\
\text { (MALDI)[86] }\end{array}$ & Laser Ablation & $10-100 \mu \mathrm{m}$ & $600 \mathrm{~nm}[80]$ \\
\hline $\begin{array}{l}\text { Secondary Ion Mass Spectrometry } \\
\text { (SIMS)[83] }\end{array}$ & Ion Beam Ablation & $200-700 \mathrm{~nm}$ & $100 \mathrm{~nm}[82]$ \\
\hline
\end{tabular}

Table 1-2 Imaging ionization techniques, based on type, commercially available resolution, and highest reported resolution.

Of these techniques, the most common imaging technique is MALDI, which is robust, tunable, and reasonably reproducible, and second only to SIMS in spatial resolution. It has an advantage over SIMS in being a "soft" ionization technique, though SIMS has seen a renaissance in method by replacing hard metal ion beams with softer, but significantly larger, gas and water cluster beams[95-97]. As another edge on SIMS, MALDI experimentation is rapidly reaching SIMS 
spatial resolution (600 $\mathrm{nm}[80]$ vs $100 \mathrm{~nm}$ [82]) while maintaining lower rates of fragmentation. It is also the core focus of my work within lipidomics, owing to the broad applicability of technique to lipidomics and the abundance of tools now available to tune experimental design.

The main hurdles in the use of MALDI for lipidomics have been widely analyzed, but fall under a few main concerns: tissue preparation, matrix choice, matrix application, and ionization efficiency. Tissue preparation issues are not unique to MALDI, as all MSI techniques require a useful substrate. Unlike histological staining techniques (hematoxylin and eosin[98], immunohistochemistry[99], oil red O[100], etc.) which can operate with formalinfixed, paraffin embedded (FFPE) tissue[101, 102] or with cutting aids (OCT[103]), MALDI tissues are generally required to be fresh frozen only, which can generate issues in sample storage stability and section-to-section variability. However, such tissues are also much closer to their "native" environment and avoid delocalization of analytes as often happens during tissue washing and preparation.

Matrix choice is just as important in MALDI as it is in ESI, except in the fact that it acts as both lipid extraction and ionization choice simultaneously. These matrices are small, typically volatile molecules that absorb specific wavelengths of light very well, with most vacuum-based methods using wavelengths between 280 and $355 \mathrm{~nm}[104]$. They assist in the ionization of analytes of interest, either ionizing first itself and then transferring that charge to the surrounding tissue or aiding in the desorption of already charged ions from the surface. The exact mechanism is still unknown at this point in time[105-112], but it has been shown that different matrices with different proton affinities produce different ions[113115]. Some matrices are great proton donors[113], others good proton scavengers[114], or both[115], and still others can chemically bind to analytes of interest[116-118]. It is abundantly clear that this is an area of intense, active research.

The method by which a MALDI matrix is applied can be just as important as which matrix is chosen. The more "wet" the method is, the greater the extraction of lipids from the tissue tends to be[119]. As such, spray-based methods have long been the workhorse for MALDI lipidomics, varying from hand-held sprayers modified from airbrushes to automated commercial sprayers[120]. These tend toward relatively high yield of analytes and can be adjusted to optimize for different lipids (either polar or neutral). However, they are often prone to operator 
differences (especially for hand-held devices), and the wetter a method is, the greater the delocalization of analytes. Similarly, spray-based matrix application lends itself to crystal sizes from 10-100 $\mu \mathrm{m}$, depending on how slowly the solvent evaporates. As a consequence, the matrix crystals can become the limiting factor in spatial resolution of MALDI imaging, as the difference in ionization efficiency between tissue and tissue+matrix can be staggering[121]. This has led to the use of sublimation-based methodologies in high spatial resolution imaging, which produces much finer crystal sizes (potentially to less than $1 \mu \mathrm{m}$ ) and minimizes delocalization of lipids within the tissue[122, 123]. A further refinement of sublimation has been the addition of a "recrystallization" step[124, 125], wherein the matrix is applied first, and then exposed to a high-humidity environment, which causes the crystals to grow and absorb additional analytes. While the size of crystals increases in doing so, typically by a factor of 2-3, most commercial MALDI instrumentation is still above the size of the expanded crystals, making it a worthwhile trade-off.

Ionization efficiency is a significant issue within the mass spectrometry imaging community, as it varies depending on the matrix proton affinity, the instrument polarity, and the extraction efficiency of the analyte into matrix[126]. As an example, in positive mode analyses of analytes with higher proton affinities than the matrix, somewhere between 1 in 1,000 and 1 in 10,000 analytes will ionize[108, 127-130]. In the case of analytes with lower proton affinities, the efficiency drops to as low as 1 in 100,000,000[131, 132]. An obvious consequence of this is a drive to improving ionization efficiency through new matrix application protocols[119, 133, 134], novel matrices[135-138], derivatization (covalently binding a charge to the analyte)[139-143], and post-ionization[144]. Matrix application, post-ionization, and matrix choice are significant factors in my quest to understand the lipidome.

\section{MASS ANALYZERS}

Once ions have been generated, we have to separate them from one another before they can be detected. The second part of a mass spectrometer is the mass analyzer, which is usually the defining characteristic of a mass spectrometer, with resolution, accuracy, and analysis speed being the 3 major concerns for end-users. Focusing on resolution first, we define it as the nominal mass-to-charge $(\mathrm{m} / \mathrm{z})$ of the peak divided by the width of the peak $(\Delta \mathrm{m})[145]$. Of the types of mass analyzers 
that see common use in lipidomics, they range in mass resolution from as low as 1,000 to greater than $1,000,000$, though within the lipid range $(600-900 \mathrm{~m} / \mathrm{z})$ it is most common to see values between 5,000 and 100,000. Mass accuracy, defined as the ratio of the $m / z$ measurement error to the true $m / z$, is second only to the resolution in importance. Generally mass accuracy is defined in parts-per-million (PPM), i.e., that at a $m / z$ of $1,000,000$ a 1 ppm error would be 999,999 or $1,000,001$ and scaling with $m / z$. In long experiments, low mass accuracy will eventually erode the mass resolution, as small errors build up into greater errors. Analysis speed is a growing concern within the field, due to the increase in spatial resolution available with modern ionization techniques. For MALDI this is especially important, as most matrices are volatile, and lengthy experimental times couple poorly to the gradual loss of the matrix.

In terms of mass resolution, analyzers at the lower end are typically quadrupolar in design, including the quadrupole mass analyzer and the ion trap, with the latter having seen multiple designs in its decades of service[146-151]. These analyzers work by scanning a voltage (typically radiofrequency), which causes packets of ions above a given $\mathrm{m} / \mathrm{z}$ to destabilize and eject from within the trap. Increased resolution is achieved by scanning more slowly, with mass resolutions above 10,000 requiring several seconds[152]. However, despite relatively high mass resolution being available through ion traps, the mass accuracy of such devices shows only poor conversion from higher resolutions. This leads to the use of quadrupoles as one stage in hybrid mass spectrometers (to be discussed momentarily), instead. The lion's share of lipidomics work performed instead of time-of-flight (TOF) instruments. TOF instruments easily reach mass resolutions of 60,000-80,000, and operate with duty cycles enabling commercial instrumentation to reach 50 pixels per second[153]. However, they are non-mass selective, require high voltage, higher vacuum than ion traps, and their mass accuracy is dependent on the instrumental geometry. Despite these flaws, they are extraordinarily attractive mass analyzers, especially when used in hybrid instrumentation or reflectron geometries. The final two mass analyzers operate using Fourier transforms to convert cyclic ion motion into $\mathrm{m} / \mathrm{z}$ information[154, 155]. Of these, the older technology is the Fourier Transform Ion Cyclotron Resonance (FTICR) analyzer, which functions by measuring the cyclotron motion of ions in a fixed magnetic field. As of my $\mathrm{PhD}$, this is the analyzer with both the highest mass resolution and mass accuracy, achieving arbitrarily high mass 
resolution alongside parts-per-billion mass accuracy[156]. As a trade-off, FTICR instrumentation requires ultra-high vacuum, superconducting magnets, cryogenic fluids (including liquid helium), and potentially significant post-processing. Further, FTICR scan times are significantly longer than TOF scans, and typically have severe data storage requirements. The other Fourier transform instrument, the Orbitrap, shares many features with FTICRs. Very high resolution $(1,000,000$ at $\mathrm{m} / \mathrm{z} 200$ ), relatively long scan times ( $\sim 3 \mathrm{~s}$ to achieve such resolution), high mass accuracy $(<1 \mathrm{ppm})$, and temperature sensitivity. Orbitrap instruments are still lower in resolution and mass accuracy than the best FTICRs (though they are approaching parity in the lipid mass range[157]), but do not require cryogenic fluids to operate.

\section{Hybrid MASS SPECTROMETERS AND TANDEM MASS SPECTROMETRY}

Identifying the elephant of a single lipid requires more than just an exact mass (Figure 1.3). Sufficiently high mass resolution can identify the sum composition of a lipid but can still fail to completely separate isobaric compounds (ions with the same nominal mass) or isomers (ions with the same sum composition in a different arrangement). As an example, $[\mathrm{PC} 36: 1+\mathrm{Na}]^{+}$and $[\mathrm{PC} 38: 4+\mathrm{H}]^{+}$are isobaric, and differ from one another by $2.4 \mathrm{mDa}$, or $0.0024 \mathrm{~m} / \mathrm{z}$. Separating these requires a mass resolution of 337,000, which is impossible for non-FT instrumentation. Even if you could separate these two isobars, [PC $36: 1+\mathrm{Na}]^{+}$is indistinguishable from the isomeric $[\mathrm{PE} 39: 1+\mathrm{Na}]^{+}$. 

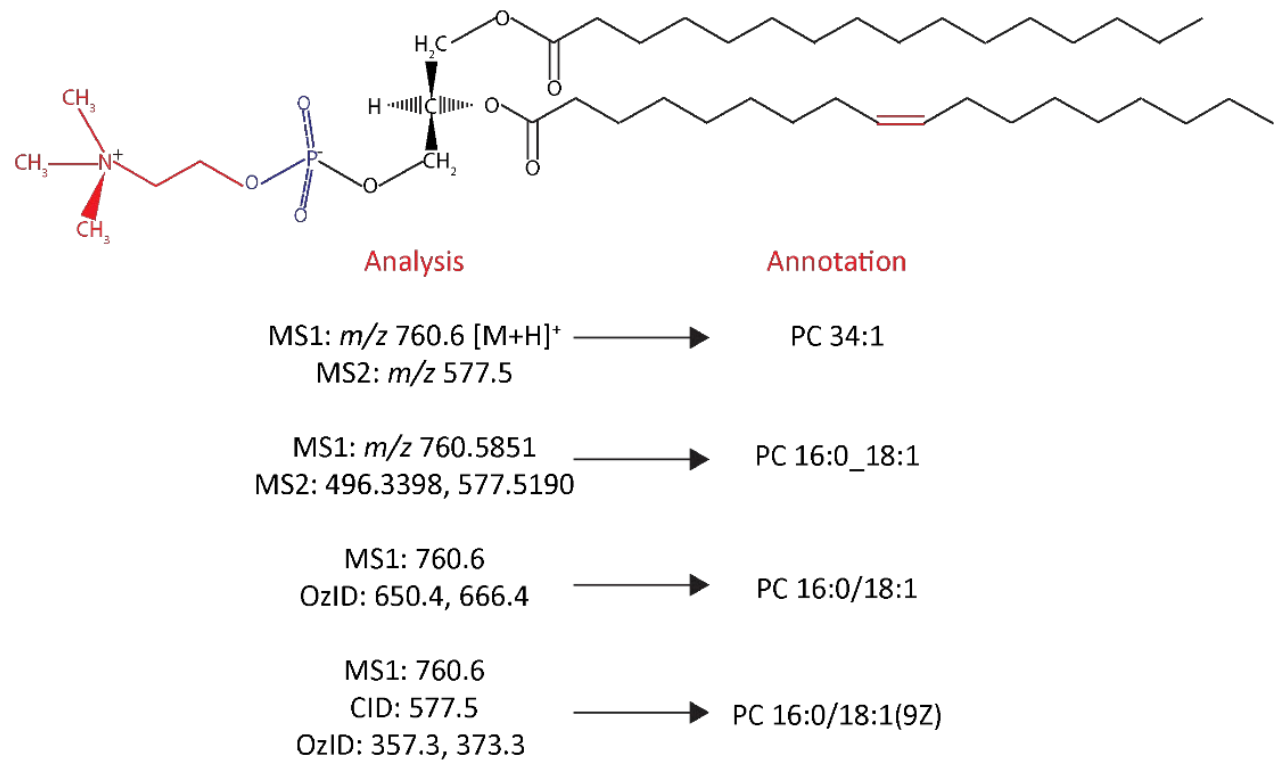

Figure 1.3. Analytical method needed for hierarchical identification of a representative phospholipid.

Enter tandem mass spectrometry. By combining mass analyzers, most typically a quadrupole with any other mass analyzer, it becomes possible to selectively fragment ions. The most widely used is Collision Induced Dissociation (CID), which exposes the selected ions to a neutral gas, with the resultant energy in the collision fragmenting the ion[158]. The fragments are then analyzed in the second mass analyzer as they would be in a normal experiment. As the fragmentation of lipids follow predictable pathways, the neutral losses (pieces that broke away without charge) and daughter ions (the fragments that retain charge) can be used to reconstruct the original ion. In the GPLs, the phosphate headgroup is both readily fragmented and highly specific, making identification of the GPLs by phosphate group straightforward. In the elephant metaphor, this would be the ability to distinguish an African from an Asian elephant. CID does suffer from a $\mathrm{m} / \mathrm{z}$ cutoff, which is about $1 / 3$ of the $\mathrm{m} / \mathrm{z}$ of the parent ion. All daughter ions below this cutoff are lost, which can include diagnostic fragments. Certain fragmentation methods, including high-energy CID (HECID) and higher-energy collisional dissociation (HCD) can generate more and different fragments that alleviate this issue, and HCD specifically has no low-mass cutoff[159].

For mass spectrometry imaging, which lacks separation methods prior to ionization, identifying the acyl chains in a lipid can be a significant challenge. There are, however, multiple dissociation techniques that can be used to overcome 
these challenges: ozone-induced dissociation (OzID)[160], ultraviolet photodissociation (UVPD)[161], ion-ion reactions[162], epoxidation[163], and the Paterno-Buchi (PB) reaction[164]. These technologies selectively fragment lipids between carbon-carbon double bonds and can be combined with CID to identify $s n$ - positions as well[161, 165].

At this stage, lipids can be uniquely identified in all respects save two: double bond geometry and chirality of the glycerol backbone. Some evidence in LC-MS work indicates that those barriers are not insurmountable[166, 167], but the application to MSI is, sadly, still lacking.

\section{SPATIAL OMICS}

While the work involved in this thesis is impactful on its own, it is only a single portion of the greater field of spatial omics. Spatial omics expands out from this work in two directions: first, that it encompasses more than just lipids under its purview and second, that mass spectrometry, imaging or otherwise, is only a portion of what can be used to understand health and disease. The idea of applying omics level identification of the transcriptome, lipidome, metabolome, proteome, etc. in an imaging level modality is both exciting and daunting, representing the next step forward.[168] My work in this thesis scratches at the surface of multiomics studies, incorporating lipidomic analyses, with tissue staining and non-lipid masses offering supporting evidence. It is a meager representation of the full scope of a spatial omics workflow, but I have contributed to such endeavors in collaboration with other scientific groups (see Other Publications, especially those with Queensland University of Technology and the Technical University of München).

\section{ADVANCING AT THE BORDERS}

Lipidomics is a broad field, encompassing both the identification of specific lipids and their position within the greater context of biology. If the earth is akin to the body, not only are we trying to identify where on earth elephants live, but their exact populations and the exact species in each location. My work has been dedicated to bringing together disparate technologies with the intent to take tissues and identify their lipid components without ever losing their position. To that end, a thorough review of the field as it stood in 2017 is provided, which shall hopefully expand upon this introduction and help explain further why this was 
undertaken. From there we will improve lipid identification by sum composition using the highest mass resolution instrumentation ever applied to lipidomics imaging. Pushing spatial resolution was the next tack, which coincidentally dovetailed with improving ionization efficiency; both accomplished using MALDI2 post-ionization. Last has been a drive towards better methodologies in structural identification of lipids, trying to image lipids not just within one class but all glycerolipid classes. 


\section{Chapter 2 ADVANCES IN MASS SPECTROMETRY IMAGING ENABLING VISUALIZATION AND OBSERVATION OF LOCALIZED LIPID BIOCHEMISTRY WITHIN TISSUES}

Andrew P. Bowman, Ron M.A. Heeren and Shane R. Ellis

The Maastricht Multimodal Molecular Imaging Institute (M4I), Division of Imaging Mass Spectrometry, Maastricht University, 6229 ER Maastricht, The Netherlands 


\begin{abstract}
There are numerous reports demonstrating the heterogeneous spatial distributions of lipids throughout biology using mass spectrometry imaging (MSI). However, despite these and the biological significance of lipid alterations, there remains a surprising lack of understanding regarding the underlying origins of the lipid spatial distributions detected with MSI. With the rapid improvement in analytical capabilities of MSI instrumentation, it is now at the point that these biological questions can begin to be addressed. In this review we focus on (i) work providing insight into the biochemical origins of lipid spatial distributions, with an emphasis on the role of localized enzymatic processes in determining lipid compositions; (ii) use of stable isotope labelling to spatially-resolve the kinetics of lipid synthesis; and (iii) current challenges faced by the lipid-MSI community and new analytical methods to overcome them. Combined, these topics highlight the added biological information that can acquired from the MSI of lipids.
\end{abstract}

\title{
INTRODUCTION
}

The continuous and rapid improvements in mass spectrometry (MS) technology is strongly correlated with the increased appreciation and understanding of the critical and complex roles performed by lipids in biology[169, 170]. While the ever-improving resolving power and structural elucidation capabilities of MSbased lipidomics have shed light onto the sheer complexity of the lipidome, both with respect to compositional and structural variations, they have also highlighted the vast analytical challenges still faced[171]. To date, the majority of lipidomics studies are performed on extracts following sample homogenization. While these approaches have proven immensely valuable, they necessitate the loss of information regarding the spatial context of a lipid in a heterogeneous tissue or cell. As a consequence, information relating to the local microenvironment in which a lipid is present is sacrificed, and significant, but highly localized, lipid alterations may be diluted beyond the detection limit in the extract.

Over the last decade, mass spectrometry imaging (MSI) has emerged as a powerful analytical method to probe the spatial distributions of many lipid classes throughout biological tissues[172, 173]. A key advantage of MSI over other imaging modalities is the ability to perform unlabeled imaging in parallel, with simultaneous detection of numerous lipid species having distinct mass-to-charge 
values. From a lipidomics standpoint, one can thus think of MSI as performing a $\mathrm{MS}^{1}$-level shotgun lipidomics experiment at each pixel. This can be achieved using a variety of desorption/ionization methods, with Matrix-Assisted Laser Desorption/Ionization (MALDI)[173], Secondary Ion Mass Spectrometry (SIMS)[174] and Desorption ElectroSpray Ionization (DESI)[175] representing the most popular approaches. Lipids present a highly promising class of molecules to study by MSI, due to the high abundance and ionization efficiency of many lipid species, and to their implications in many biological processes. Furthermore, unlike other molecular classes like proteins, lipids have arguably the most straightforward sample preparation procedures, making their detection reproducible and robust[176]. By exploiting the untargeted nature of MS, it is possible to probe for induced lipid profiles within different tissues and cell types. Heterogeneity in lipid profiles is observed in virtually all MSI studies, highlighting the many and specific roles performed by individual lipid species, and the valuable information obtainable from the spatial location of lipids. This has led to the emergence of MSI as a potentially powerful diagnostic tool, capable of identifying tissue-type changes in lipid composition and making diagnostic predictions based on localized lipid composition[177]. To illustrate, changes in local lipid compositions have been shown to enable differentiation of brain tumor sub-types[178] or enunciate differences in fatty liver disease[179], while alterations in lipid profiles also show much promise for real-time annotation of tissue types during surgery[180, 181]. This approach is typical of many lipid MSI studies that seek to determine the alterations in global profiles throughout different tissue regions without necessarily needing to know the identity or implications of the detected lipids. However, to understand the biological meaning behind MSI data, identification of the lipid species is required.

Despite the ever-increasing utility of lipid MSI as an (bio)analytical technique, it is remarkable that there exists scant knowledge as to why lipids exhibit different spatial distributions throughout tissues or with a change of biochemical state (e.g., disease). In many MSI studies, accurate mass and tandem mass spectrometry (MS/MS) are performed to identify some of the observed lipids. However, due in part to a general lack of understanding regarding the roles of individual lipid species in biology, it has been difficult to interpret lipid spatial distributions in terms of biological processes. To move beyond simply visualizing altered spatial distributions, it is becoming increasingly important to understand the 
biochemical origin of lipid MSI results. This would enable the researcher to place images into a biological context and understand both the cause and consequence of spatially altered lipid compositions. With advances in sensitivity, spatial resolution, and chemical specificity, MSI technology is now at the point where it can begin to address these paramount questions. In this review, we describe MSI work focusing not only on lipid spatial distributions, but also on beginning to elucidate the underlying processes giving rise to lipid MSI data. We also describe advances in MSI technology that provide new opportunities for MSI (e.g., kinetics, improved lipidomics coverage, sensitivity, and structural specificity) along with critical analytical challenges that must be addressed to enable imaging of structurally defined lipid species (i.e., true molecular imaging).

\section{ANALYTICAL METHODS FOR MSI}

In this section we briefly describe relevant analytical aspects of the key desorption/ionization techniques used for lipid MSI.

\section{MALDI}

MALDI is the most prominent method used for lipid MSI. It offers high sensitivity for many important lipid classes, and can be coupled to most mass analyzers, enabling both high mass resolution and structural analysis via MS/MS of detected lipids. The requirement for a matrix compels careful consideration in regards to sample preparation, especially if high spatial resolution is required [182], and several protocols have been described for popular matrices [176, 183]. The classes of lipids detected can be somewhat tuned via matrix selection; however, in most cases positive-ion mode spectra are dominated by Phosphatidylcholine (PC) and Sphingomyelin (SM), while negative-ion spectra are dominated with acidic phospholipids and sphingolipids (e.g., sulfatides) [173]. Although termed a "soft" approach, MALDI can still result in some in-source fragmentation which can lead to erroneous assignment of detected ions, such as endogenous phosphatidic acid (PA), dehydrated Diacylglycerides (DAG) or free Fatty Acids (FA) as real peaks, rather than readily formed fragments of phospholipids. Commercial MALDI MSI instruments now offer throughput approaching 50 pixels/second and spatial resolutions down to $10 \mu \mathrm{m}$, while experimental instruments have approached the one micron realm [184].

SIMS 
SIMS offers the highest spatial resolution of any MSI technique. Using traditional atomic ion beams, spatial resolutions as low as several hundred nanometers have been achieved for lipid imaging [174, 185]. However, the resulting high energy desorption/ionization process results in severe fragmentation, making intact lipid species difficult to observe and impairing true lipidome imaging, as well as hindering interpretation. This has been partly addressed with molecular beams, such as $\mathrm{C}_{60}{ }^{+}$, which offer softer ionization conditions and enable the detection of intact lipid species [174]. When deployed on instruments with direct current beam operation and continuous ion generation, spatial resolutions as low as $300 \mathrm{~nm}$ have been reported [186]. More recently, gas cluster ion beams (most commonly argon) have further improved the analytical capability of SIMS for lipid MSI [187]. These offer the softest ion generation conditions of any SIMS approach, but are to-date limited to spatial resolutions in the low micron range and can still result in greater fragmentation compared to MALDI [187]. Thus, consideration of these effects is essential to avoid misinterpretation of fragment ions as biologically significant lipids. Nonetheless, the ability to generate intact lipid ions from tissues has led to the development of SIMS identification approaches using both MS/MS and high mass resolution systems [188-190].

\section{DESI}

DESI is the most widespread ambient ionization technique used for lipid MSI [191]. This technique facilitates MSI at atmospheric pressure without the need for an external matrix, as required for MALDI. It is a soft ionization method (similar to ESI), enabling detection of a broad range of lipid classes with minimal fragmentation. Additionally, it can be coupled to a variety of mass analyzers enabling high mass resolution and MS/MS for lipid identification. The typical spatial resolution is $100-250 \mu \mathrm{m}$, which is sufficient to resolve many distinct tissue regions characterized by different lipid compositions [192]. A unique aspect of DESI is the ability to perform in-situ derivatization during the desorption/ionization step, which can enable detection of poorly ionized lipid classes such as cholesterol [193].

\section{APPLICATIONS OF MSI TO LOCALIZED LIPID BIOCHEMISTRY}

Many lipid MSI studies have focused on elucidating the altered lipid metabolism within cancers. In a MALDI MSI study utilizing Fourier Transform 
Ion Cyclotron Resonance (FTICR) mass analysis of six different types of cancer (breast, lung, colorectal, esophageal, gastric, and thyroid), Guo et al. observed elevated signals from monounsaturated lipids (MUL) [194]. All six cancer types across more than 100 different patients demonstrated a conserved set of lipid alterations, including PCs 32:1, 34:1, and 36:1 showing elevated signals in cancerous tissue relative to their saturated analogues. Interestingly, different cancers were found to overexpress specific MUL relative to others. For example, the $\mathrm{PC}(32: 1): \mathrm{PC}(32: 0)$ ratio was increased in all cancer types except thyroid cancer, whereas the PC(36:1):PC(36:0) ratio was elevated in all cancers except colorectal cancer (Figure 2.1a). Concomitantly, polyunsaturated lipids, notably PC and Phosphatidylinositol (PI) 38:4, exhibited a marked decrease in concentration in several of the cancers. The source of these significant lipid alterations was determined by studying the associated expression of enzymes related to lipid synthesis [194]. A significant elevation in expression of fatty acid synthase, stearoyl-CoA desaturase-1 (SCD1), and choline kinase $\alpha$ in the cancers was observed and correlated well with observed MSI data (Figure 2.1b), suggesting lipid distributions were the result of localized enzymatic processes [194]. This meshes well with the understanding that SCD1 is the key enzyme of the rate limiting step in the synthesis of monounsaturated fatty acids [195]. Similar SCD1 mediated alterations in monounsaturated PC lipids relative to saturated analogues have also been reported using MSI in breast cancer [196] and, tentatively, in lymphoma [197]. In addition to changes in (un)saturation, negative-ion mode MALDI MSI has shown sulfatides to be elevated in ovarian cancers [198]. Intriguingly, the increased sulfatide levels were not homogenous within the tumor, but instead localized to the tumor epithelium. Sulfatide levels were strongly correlated with a significant increase in both GalCer synthase (11-fold) and Galactose-3-O-Sulfotransferase 1 (3.5- fold) relative to normal ovarian stromal tissue, both of which are involved in sulfatide synthesis [198]. This provides a probable insight into the underlying biochemical origin of spatially resolved sphingolipid metabolism. 


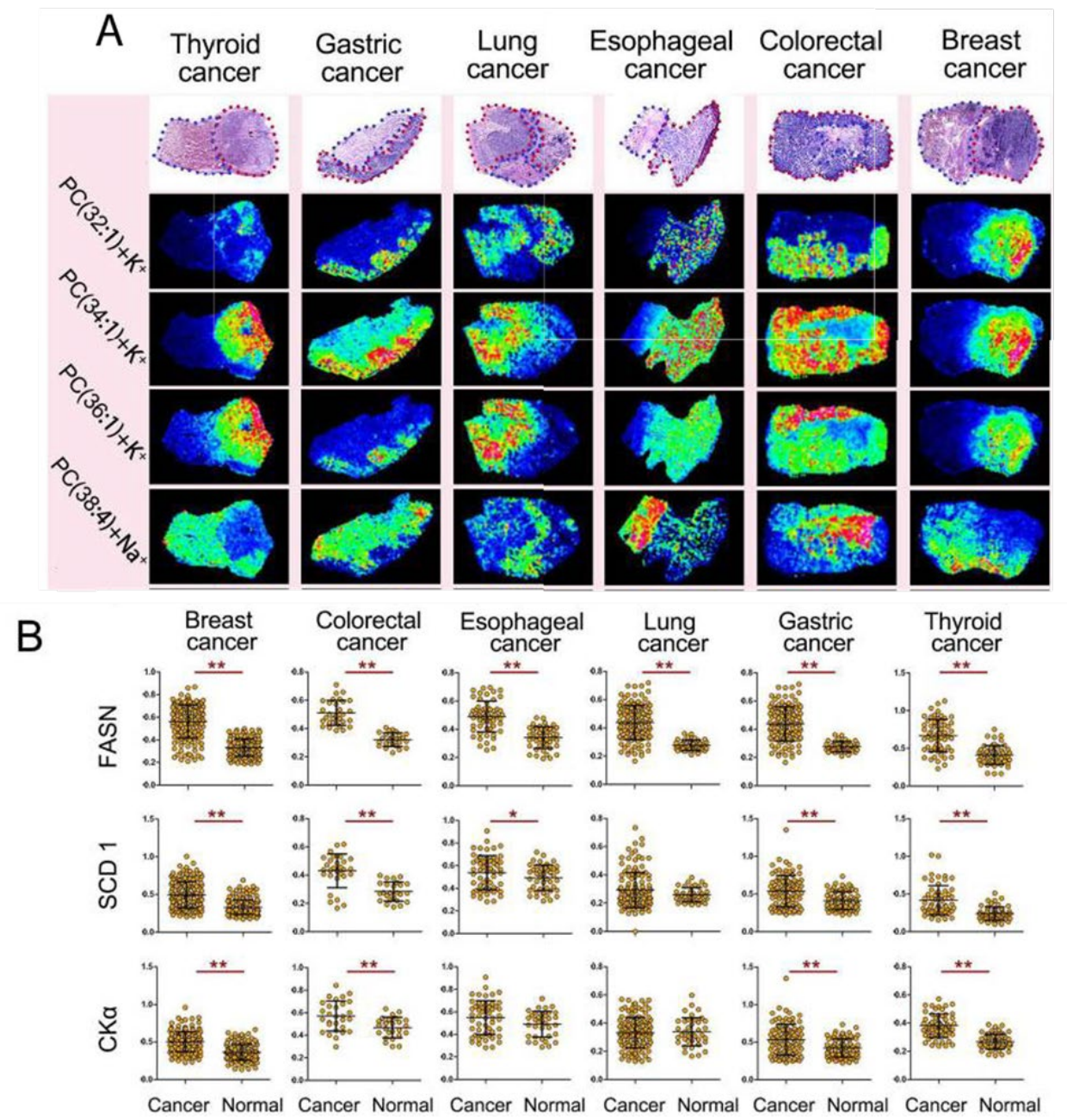

Figure 2.1 Elevated levels of monounsaturated lipids observed in various cancers by MALDI MSI at $200 \mu \mathrm{m}$ pixel size. (A). MALDI MSI data of four different PC lipid species varying in degree of unsaturation acquired from thyroid, gastric, lung, esophageal, colorectal and breast cancers. Optical images of H\&E-stained tissue sections of the six different cancers are provided above the corresponding MSI data. Cancerous areas are circled with a red dotted line and adjacent normal tissue with a blue dotted line.(B) Altered lipid compositions of cancers is also correlated with elevated levels in the expression of Stearoyl-CoA Desaturase 1 (SCD1), and Choline Kinase $\alpha$ $(\mathrm{CK} \alpha)$ and Fatty Acid Synthase (FASN), eluding to the critical relationship between localized lipid compositions revealed by MSI and enzyme processes (Image adapted with permission from reference [194]).

Enzymatic processes can also lead to the production of bioactive lipids that propagate the effects of disease/infection. In this context, several MSI studies have focused on the local synthesis and conversion of arachidonic acid containing lipids (AA-CLs). Scott et al [199] showed the breakdown of AA-CLs upon Francisella novicida infections in mice using MALDI TOF MSI and MALDI FTICR MSI. The 
infection was spatially visualized throughout mouse spleens via the infectionspecific Lipid A molecule, which was distributed throughout the red pulp and detected from 36 hours post-infection (h.p.i.) (Figure 2.2a). Spatial alterations in the host lipidome were also detected and revealed a high initial abundance of PI(16:0_20:4) in the white pulp. At 48 h.p.i., a drastic decrease in PI(16:0_20:4) was observed and associated with the onset of sepsis breakdown of the white pulp and necrosis at 60 h.p.i. (Figure 2.2b). Similar reductions in other AA-CLs were also observed and confirmed with quantitative LC-MS/MS (Figure 2.2C). The decrease in AA-CLs was shown to be the outcome of AA detachment from phospholipids via an increased activity of cytosolic phospolipase-A2 in the infection (Figure 2.2D). Critically, this release of AA was associated with significant elevation in the cycloxegenase-2 pathway (Figure 2.2E), leading to the formation of the highly inflammatory eicosanoid prostaglandin E2 (quantified by LC-MS/MS). 
$\mathbf{A}$

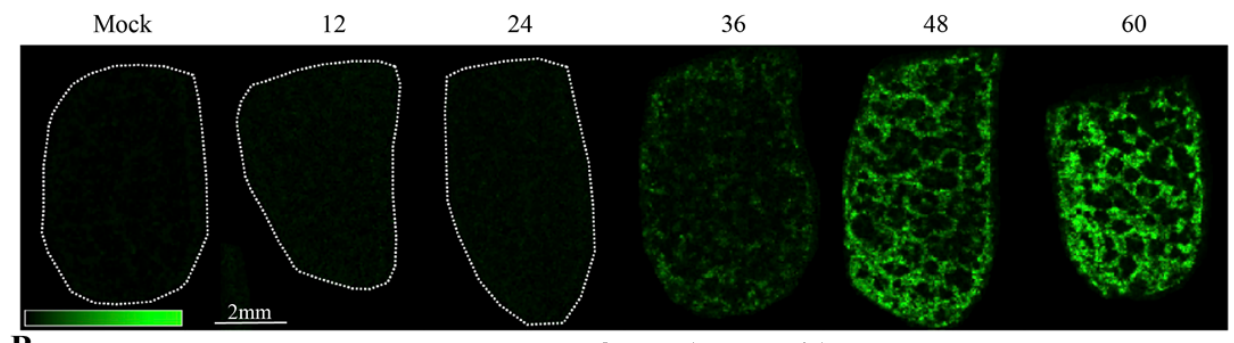

B

Hours Post-Infection (F. novicida)
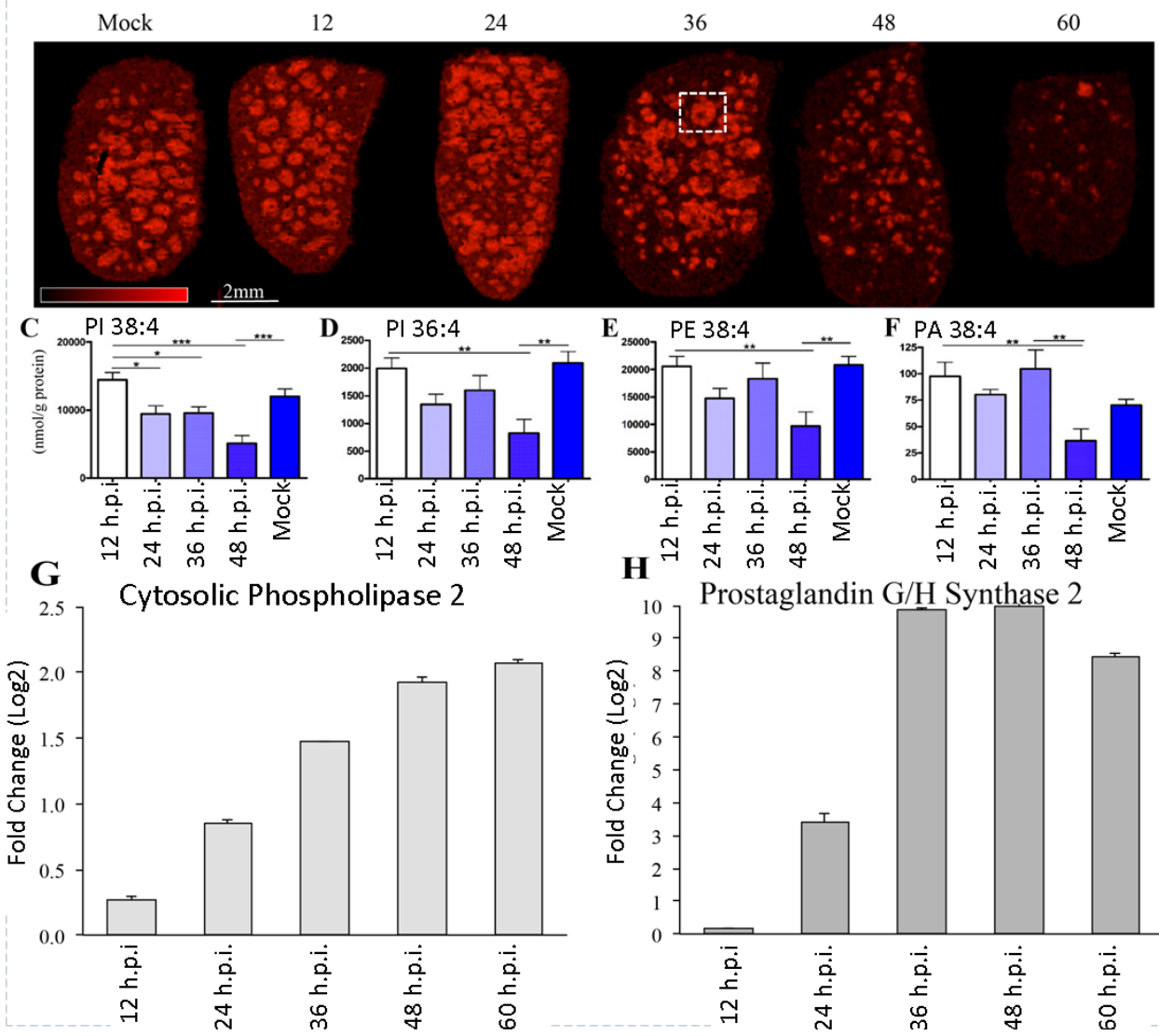

Figure 2.2 Influence of enzymatic degradation and conversion of MALDI MSI analysis (75 $\mu \mathrm{m}$ pixel size) of Francisella novicida infected mice spleen. (A) MALDI TOF MSI revealing the spatial distribution of deprotonated lipid A detected at a nominal $\mathrm{m} / \mathrm{z} 1665$ at various times post-infection (0-60 hours) (B) Corresponding spatial distribution of deprotonated $\operatorname{PI}(16: 0 / 20: 4)$ detected at $\mathrm{m} / \mathrm{z}$ 885.6. With increasing time post-infection, the increasing levels of infection-specific Lipid A is correlated with a dramatic reduction in $\operatorname{PI}(16: 0 / 20: 4)$ intensity and a loss of tissue structure. (C) Quantitative LC/MS of a variety of arachidonic acid containing lipids, all exhibiting a decrease in concentrations with infection progression. Changes in host lipid composition are correlated with increased expression of Cytosolic Phospholipase 2 (D) and Cyclooxygenase 2 (E), which result in the release of arachidonic acid from host lipids and conversion into proinflammatory prostaglandin E2, respectively. Image adapted with permission from reference [199]). 
This dramatic and rapid generation of inflammatory eicosanoids is a major contributor to the lethality of the infection. Furthermore, 3D-MSI, using highthroughput MALDI TOF systems, was used to generate a 3D model host-pathogen lipid interface, via the distribution of infection- and host-specific lipids [199]. Similar breakdown products from AA-CLs have also been implicated in Nonalcoholic Fatty Liver Disease. In a MALDI MSI study by Hall et al. distinct zonation of PC and SM lipids in nonalcoholic fatty liver were observed in disease progression, before subsequent loss in the advancement to nonalcoholic steatohepatitis [179]. AA-CL signals, in particular $[\mathrm{PC}(36: 4)+\mathrm{K}]^{+}$and $[\mathrm{PC}(38: 4)+\mathrm{K}]^{+}$, were elevated in zone 3 of the liver and strongly correlated with the location of Lysophosphatidylcholine Acyltransferase 2, a key enzyme involved in phospholipid remodeling and the creation of AA-CLs, with the localization confirmed via immunohistochemistry. Moreover, proinflammatory eicosanoids were again observed and associated with release of free AA from phospholipids following the action of cytosolic phospholipase A2 and lipoxygenase related Alox 15 enzymes. The importance of AA-CL's has also resulted in their spatial alterations being observed in several other MSI studies, including a MALDI MSI study detailing elevated levels of [PI(18:0_20:4)-H] $]^{-}$at the outer edges of colorectal cancers [200]. In another study, a logarithmic decrease in AA-CLs signals was observed from the base to the top of colon crypts and again correlated with local expression of various enzymes involved in lipid synthesis, breakdown, and metabolism [201]. Other examples of altered AA-CL distributions include an aggregation of AA-containing PC lipids near the site of spared nerve injuries, which correlated with microglia activation [202], and an observed gradient of AAcontaining PCs in the axons of mice neurons, with enriched levels observed within the axon and decreasing proximal-to-distal [203].

Alterations in sphingolipid metabolism have been studied using MALDI MSI, both liver and kidney with tissues sourced from wild type and sphingomyelin synthase 2 (SMS2) deficient mice when fed either a normal or high fat diet [177]. SMS2 is a key enzyme involved in the conversion of ceramides into SM. In all mice, potassiated $\mathrm{SM}(\mathrm{d} 18: 1 / 16: 0)$ was localized at the border of the renal cortex and medulla. In contrast, $[\mathrm{SM}(\mathrm{d} 18: 1 / 22: 0)+\mathrm{K}]^{+}$was localized in the medulla, with lower levels observed in the SMS2-knosckout mice [177]. Moreover, $[\mathrm{SM}(\mathrm{d} 18: 1 / 24: 0)+\mathrm{K}]^{+}$and $[\mathrm{SM}(\mathrm{d} 18: 1 / 24: 1)+\mathrm{K}]^{+}$were localized in the renal cortex 
and exhibited reduced levels upon high fat diet and SMS2 deficiency, thus alluding to a role of SMS2 in region-specific SM synthesis in the kidney.

Non-polar lipids, such as triacylglycerides (TAGs), have also been studied with MSI. In one SIMS study, a $\mathrm{Bi}^{3+}$ ion beam was used to probe intestinal absorption of dietary fat in mice, via analysis of their duodenums after different digestion times following force feeding with cholesterol-enriched sunflower oil [204]. Comparisons of peak ratios following different digestion times revealed selective alterations in certain lipid species. For example, peak ratios of C18containing Monoacylglyceride (MAG), DAG, and TAG signals increased with digestion time. Spatially, lipid signals corresponding to those species present at high levels in the oil (C18:2 and C18:1) increased mostly in the enterocytes (likely present as cytosolic lipid droplets), while C18:0, C16:1 and C16:0 carboxylate signals decreased in the lamina propria [204]. Isolation of cytosolic lipid droplets was performed, with neutral lipid composition confirmed with gas-liquid chromatography. Activity of acyl-transferase enzymes was also detected (MAG acyl transferase 2 and $D A G$ acyl transferase 2), in addition to other enzymes including acyl-coenzyme A, cholesterol acyltransferase and adipose triglyceride lipase. This suggests that the SIMS MSI data, which showed localized uptake of dietary lipids, can be rationalized via enzymatic-driven metabolic processes.

MSI has also been used to study lipid metabolism in plant seeds. Using MALDI MSI, Horn et al described the acyl-chain dependencies of lipid distribution in the oilseeds of Camelina sativa [205]. As an example, 18:2 containing lipids, such as PC(16:0_18:2) and PC(18:2/18:2), were enriched within the embryonic axis relative to cotyledons. In contrast, PC lipids containing 18:3 or 20:1 chains were enriched in the cotyledons relative to the embryonic axis. A variety of genetically engineered oil seeds, which were enriched in 16:0, 18:1 and 18:2 fatty acids, were produced via interference in the enzymatic synthesis of fatty acids and subsequently studied with MALDI MSI. In 16:0-enriched seeds, PC(16:0_18:2) revealed a relatively homogenous distribution in stark contrast to the wild-types seeds [205]. Moreover, even with 16:0 making up 40\% of the available fatty acid pool, little of the fully saturated PC and TAGs (i.e., PC(32:0) or TAG(48:0) were observed, suggesting the presence of a metabolic bottleneck in the generation of fully saturated PC and TAGs. For engineered seeds enriched with 70\% 18:1, PC(18:1/18:1) showed a heterogeneous distribution, being elevated in the cotyledons while polyunsaturated PC lipids were relatively increased in the 
embryonic axis. These results suggested an incomplete suppression of fatty acid desaturase 2, with it being more active in the embryonic axis. In the context of genetically engineering oil compositions, this could infer an upper limit of 18:1 accumulation determined by compartmentalization. Such effects can only be visualized with imaging methodologies, with this study in particular demonstrating the analytical potential of using altered lipid compositions to infer localized enzymatic processes and their alterations.

Finally, in some cases one can take advantages of the fact that positive-ion mode lipid MSI often results in the observation of multiple adducts for a given lipid species (e.g., $[\mathrm{M}+\mathrm{H}]^{+},[\mathrm{M}+\mathrm{Na}]^{+}$and $\left.[\mathrm{M}+\mathrm{K}]^{+}\right)$. In MALDI studies, the ratios of adducts such as these are harder to interpret, as the chemistry of the applied matrix can create alterations during desorption/ionization step, making it imperative that changes in adduct ratios are not mistakenly interpreted as altered lipid compositions. However, changes in sodiated and potassiated ion ratios can potentially provide insight into altered activity of $\mathrm{Na}^{+} / \mathrm{K}^{+}$-ATPase activity. For example, using MALDI MSI, a relative increase in $[\mathrm{M}+\mathrm{Na}]^{+}$ion signal of $\mathrm{PC}(16: 0-18: 1)$ compared to $[\mathrm{M}+\mathrm{K}]^{+}$ion has been observed at the site of traumatic brain injury [206], while altered $\mathrm{PC} \mathrm{Na} / \mathrm{K}^{+}$ratios have also been observed in livers following hepatitis $\mathrm{B}$ virus (HBV) infected livers with HBV-mediated regeneration defects [207].

\section{ADDING TEMPORAL DATA: VISUALIZING LOCAL LIPID DYNAMICS IN TISSUES}

Despite the vast potential of MSI to study alterations in localized lipid compositions throughout tissues, it is still blind to a critical aspect of lipid biochemistry; that of the dynamic nature of in-vivo lipid synthesis and turnover. A powerful approach to access this dynamic information lies in stable isotope labelling, as employed in flux-based lipidomics studies [208]. By incorporating stable isotopes involved in lipid synthesis into a biological environment, their uptake into various lipids can be detected with MSI. Although the use of stable isotope labelling combined with MSI has found success in SIMS, in particular nano-SIMS which enables sub-cellular imaging of isotopic enrichments, these studies are limited by the extensive fragmentation induced by the desorption/ionization step (e.g., detection of $\mathrm{CH}^{-}$or $\mathrm{CN}^{-}$) meaning only global enrichments can be measured. It is only recently that soft-ionization MSI methods 
been deployed to study lipid dynamics of intact (and thus identifiable) lipid species [187].

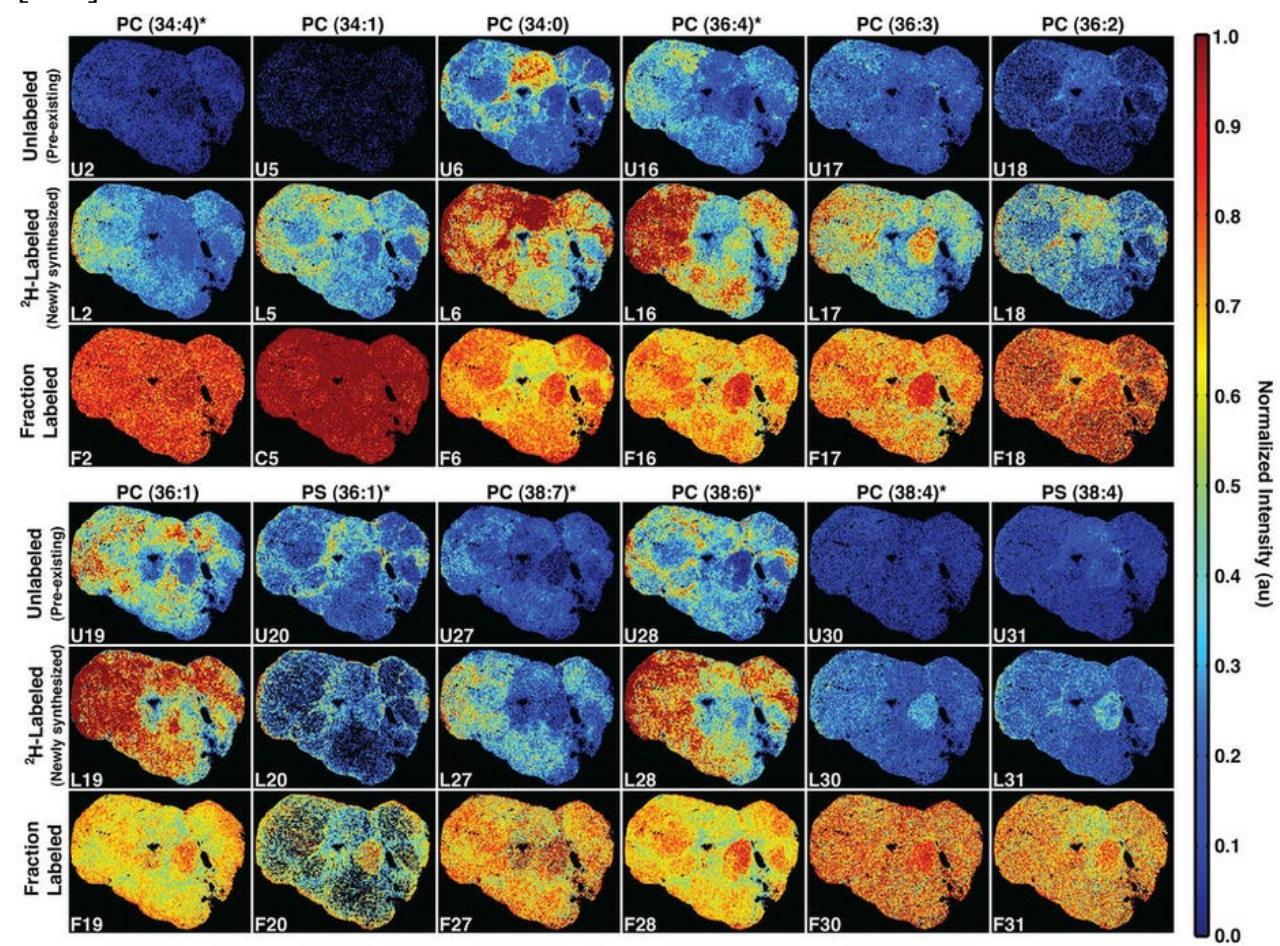

Figure 2.3 Use of isotope stable isotope labeling combined with MSI to reveal the kinetics of lipid synthesis (deuterium labelling via heavy water consumption in this example). (Row 1, 4) MALDI MSI data (50 $\mu \mathrm{m}$ pixel size) of various unlabeled lipid species (lipid species annotated above the corresponding column). (Row 2, 5) Corresponding spatial distributions of deuterium enriched (newly synthesized) lipid species. (Row 3,6) ratio images of labelled:unlabelled lipid species showing relative synthesis rates of various lipids in each pixel (higher intensity equates to faster synthesis). Image used with permission from reference [209].

Two studies have applied MSI to study the kinetics of phospholipid synthesis following $\mathrm{D}_{2} \mathrm{O}$ administration $[192,209]$. In both studies, drinking water was enriched with $8 \% \mathrm{D}_{2} \mathrm{O}$, resulting in the incorporation of deuterium into newly synthesized lipids. In the study by Louie et al, mice containing breast tumors were provided access to $8 \% \mathrm{D}_{2} \mathrm{O}$ drinking water for 5 days prior to sacrifice and collection. Analysis of tissue was performed using nanostructured initiator mass spectrometry, which can be viewed as an alternative to MALDI where the surface acts as the matrix. The resulting per-pixel TOF spectra provided both lipid compositions (mostly PC lipids) and information regarding local synthesis by shifted isotope profiles [209]. Distributions of a variety of unlabeled PC lipids are provided in Figure 2.3 (row 1 and 4) revealing heterogeneous endogenous lipids 
throughout the tumor tissue. Interestingly, MSI of the corresponding deuterium enriched lipid signals (Figure 2.3 row 2 and 5) and fractional enrichment values (Figure 2.3 row 3 and 6 ) reveal different ion distributions that likely reflect tissue and cell-type specific variations in lipid flux. In a similar study performed with DESI and rat brain tissue, subtle regio-specific alterations in the synthesis of several lipids, including PS(40:6) and PI(38:4) were observed [192]. For example, faster synthesis of PS(40:6) was detected in the cerebral cortex and the septal nucleus. In contrast, $\mathrm{PI}(38: 4)$ revealed the highest turnover in the corpus callosum, where the overall concertation is lowest, providing evidence for either rapid degradation/conversion or redistribution throughout the brain. A potential disadvantage of $\mathrm{D}_{2} \mathrm{O}$ labelling is the high probability that a given lipid species can possess a distribution in the number of deuterium atoms. In the absence of sufficient mass resolution, this can invariably result in isobaric overlaps and obfuscates signals related to low-abundance lipid species. In an alternative approach, $\mathrm{d}_{3}$ palmitate was used to study the kinetics of PC lipid synthesis within neurons cultured in a compartmentalized system [210]. Using high spatial resolution MALDI MSI ( $6 \mu \mathrm{m}$ pixel size), the incorporation of labelled palmitate could be visualized across individual neurons. The most abundant labeled lipid signals corresponded to PC(32:0) containing two $\mathrm{d}_{3}$-palmitate acyl chains. Applying $\mathrm{d}_{3^{-}}$ palmiate to the loading side of the culture, labelled $\mathrm{d}_{6}-\mathrm{PC}(32: 0)$ was observed around the cell body and also within the neurites along the microgroove regions of the culture. When $d_{3}$-palmiate was only added to the distal side, $d_{6}-\mathrm{PC}(32: 0)$ was only enriched in the tips of the neurites [210], suggesting that transport of newly synthesized lipids from the distal side throughout the neuron is restricted.

The above reports have only just begun to scratch the surface of the possibilities enabled by kinetic-MSI coupled with isotope labeling, and it is certain to become a rapidly growing area for MSI in the near future, providing a window into a thus far hidden dimension of biochemical information and is expected to grow in popularity. It is likely that, to fully exploit isotope labelling, high mass resolving power MSI will be required to resolve (insofar as is possible with current high resolving power MSI instruments) isobaric ions that will inevitably be present due to both the diversity of naturally generated lipids and those enriched with stable isotopes (i.e., to resolve $[\mathrm{PC}(34: 1)+\mathrm{H}]^{+}$containing $2 \mathrm{x}$ deuterium or carbon-13 atoms from monoisotopic and $\left.[\mathrm{PC}(34: 0)+\mathrm{H}]^{+}\right)$. Regarding enrichment sensitivity, with current MSI technology it is reasonable to expect the ability to detect and 
image enrichment factors of $\sim 1 \%$ for many species in a single pixel, which is roughly an order of magnitude higher than required with current extract-based methods (e.g., those based on lipid extraction from homogenized tissue) [211]. With further improvements in sensitivity and dynamic range, isotope labeling strategies will provide a powerful toolbox for studying a diverse array of cell- and tissue-specific lipid synthesis and conversions processes.

\section{CURRENT ANALYTICAL CHALLENGES AND FUTURE OPPORTUNITIES IN LIPID MSI}

Lipid MSI has seen a dramatic improvement in both analytical capabilities and biological applications in the last two decades, yet there are still significant hurdles that must be addressed to enable both broad imaging of lipid classes and of well-defined lipid molecules. By and large, these issues arise from the sheer number of lipid molecules and classes present in biology. To illustrate, while some lipid classes (e.g., PC and SM in positive ion mode) possess relatively high ionization efficiencies, other important lipid classes (such as sterols and prostaglandins) are notoriously difficult to ionize with current MSI approaches. Moreover, the complexity of the lipidome introduces significant suppression effects such that the presence of certain lipid classes can impair the detection of others [212].

Lipidome complexity is partly addressed with the emergence of high mass resolution/accuracy MSI using FTICR or Orbitrap analyzers. Such approaches facilitate sum-compositional assignment of detected lipid species, but provide little structural information beyond this (e.g., composition of individual fatty acyl chains). Laser-induced post-ionization (MALDI-2) has emerged as promising way to begin to address typically low ionization efficiencies of some lipid classes [213, 214]. Combined with high resolution mass analyzers, MALDI-2 has demonstrated significant improvements in lipidome coverage and resolution during MSI, with up two-orders of magnitude improvement for several lipid classes (e.g., GalCer and PE in positive-ion mode) (Figure 2.4). Other approaches to improve lipid coverage have been built on the observation that positive and negative-ion mode MSI offers detection of complimentary lipid classes with minimal overlap. For instance, using a precision controlled scanning laser beam and MALDI, it is possible to first acquire positive-ion mode data from a tissue section, with the laser not interacting with the entire tissue surface, after which negative-ion mode data can be acquired from the same tissue section using an interlaced scanning pattern [215]. When 
applied to zebra-finch and lung cancer xenografts, it was possible to acquire both positive-ion and negative-ion mode data from a $50 \times 50 \mu \mathrm{m}^{2}$ area of tissue and merge the data from both polarities together for multivariate analyses, thus providing more comprehensive lipid coverage from a single sample analyzed with MSI. In an alternative approach, Kaya et al have demonstrated a novel method describing positive and negative-ion lipid MSI, as well as positive mode MSI of A $\beta$ peptides in a transgenic mouse model of Alzheimer's disease (tgSwe) [216]. This approach enabled the spatial correlation of multiple lipid classes and $\mathrm{A} \beta$ peptides in amyloid$\beta$ plaques. Moreover, derivatization strategies offer an exciting means to study the spatial distributions of lipid not yet detectable with current MSI approaches [217]. While not yet widely applied to lipid MSI, one report by Wu et al demonstrated using in-situ betaine aldehyde derivatization during DESI-MSI of mouse brain for the enhanced detection cholesterol and Vitamin A [193]. 


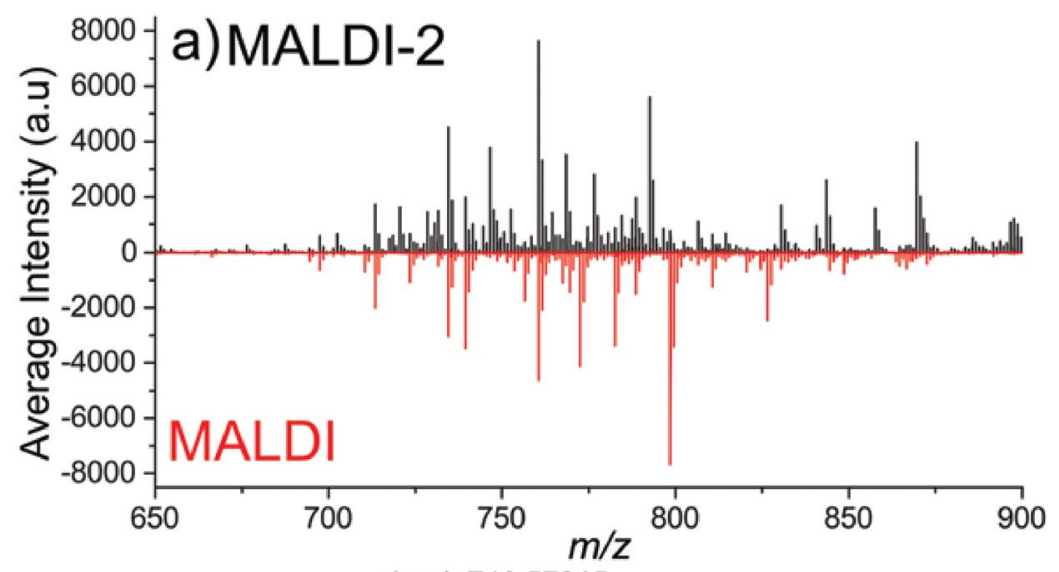

b) $m / z 792.55635$

c) $m / z 746.57015$

[PE(18:0_22:6)+H] ]

[DMPE(16:0_18:1)+H] ${ }^{+}$/
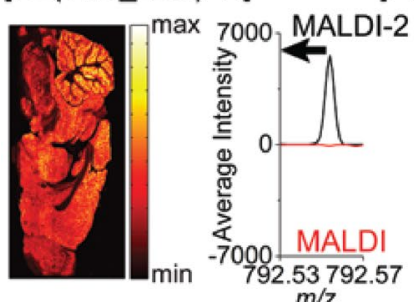

$\left[\mathrm{PE}\left(18: 0 \_18: \overline{1}\right)+\mathrm{H}\right]^{+}$

e) $m / z 798.54142$

$[\mathrm{PC}(34: 1)+\mathrm{K}]^{+}$

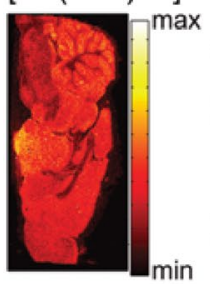

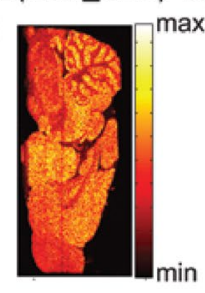

f) $\mathrm{m} / \mathrm{z} 810.68231$

[GalCer(d18:1/24:1)

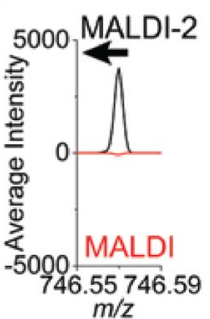

d) $\mathrm{m} / \mathrm{z} 369.35146$

$\left[\mathrm{Chol}+\mathrm{H}-\mathrm{H}_{2} \mathrm{O}\right]^{+}$
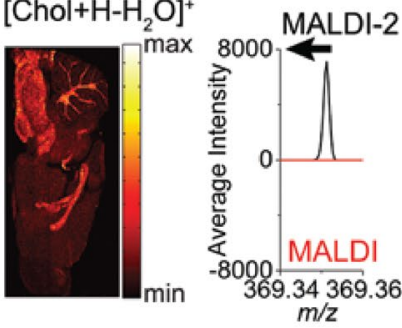

g) H\&E Image
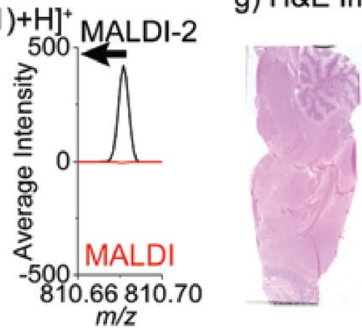

Figure 2.4 Enactment of MSI sensitivity for a variety of lipid species using laser-induced postionization coupled with MALDI MSI (MALDI-2). (A) positive-ion mode MALDI-2 (top, black trace) and conventional MALDI (bottom, red trace) spectra acquired from MSI analysis of rat brain tissue at $100 \mu \mathrm{m}$ pixel size. (B-H) The images were acquired from consecutive sections of rat brain. Corresponding ion distribution images along with the corresponding signal trace for MALDI and MALDI-2. Black arrows indicate whether MALDI or MALDI-2 data was sued to generate the shown image. PE and GalCer lipids are particular beneficiaries for positive mode MALDI2. (G) Optical image of the stained tissue sections acquired after MSI. Image reproduced with permission from reference [214].

Finally, to fully elucidate the ultimate origin of MSI-detected lipids, knowledge of the precise structure of the lipids is required. Known enzymatic processes give rise to fatty acids with defined chain lengths and double bond positions which are later incorporated into complex lipids [218]. While conventional MS/MS enables identification of acyl chain length and degree of 
unsaturation, it typically provides little information on double bond positions or positioning of fatty acyls on the glycerol backbone. Consequently, the exact identities of lipids in an MSI experiment are ambiguous. In recent years, a selection of alternative strategies such as ozone-induced dissociation [165], ultraviolet photodissociation [161, 219], photochemical Paterno-Buchi reactions [220], and ion mobility [62] have been developed to begin to unravel lipid isomers from solution-phase extracts. Although they have not been reported for isomericallyresolved MSI, these couplings provide promising approaches to being able to do so. Such techniques should enable lipid distributions to be directly correlated with the local activity of enzymes involved in their synthesis and catabolism.

\section{CONCLUSIONS}

MSI has evolved in the last two decades from a molecular imaging technique in its infancy to a potential powerhouse method for lipid analysis. However, as discussed in this review, it is only recently that insights into the underlying biochemical origin of lipid spatial distributions have started to coalesce. In-line with general knowledge of lipid biochemistry, it is apparent that to understand the biological context of lipids, lipidomics alone is often not enough. In particular, the role of localized enzyme compositions and processes has been heavily implicated as a key determinant of both lipid configuration and spatial distributions within tissues. Such multimodal information is likely a necessity to understand the cause and effect in lipid composition within tissues. However, lipids should not only be viewed as the end-products of enzymatic processes. Lipid composition is also determined by the local availability of molecular building blocks, which can be influenced via a variety of factors (i.e., diet or medications) and can be directly correlated to disease pathology. Additionally, while lipids themselves can participate in a multitude of metabolic/signaling processes and strongly influence membrane properties.

With ever increasing analytical performance, the ability to acquire new spatially resolved lipidomics information has also emerged. This includes isotope labelling studies enabling localized lipid dynamics to be probed, as well as advances in desorption/ionization methods permitting enhanced sensitivity and broader detection of lipid classes during MSI experiments. Such increased lipid coverage is essential to understand the spatial correlations amongst different, structurally related lipid species. In the near future, we envisage adoption and 
adaptation of methods originally developed for extract-based lipidomics which empowered enhanced structural resolutions. This is a key step towards overcoming the current ambiguity in MSI lipid annotations and will facilitate the imaging of structurally defined lipid molecules. Such advances will be essential to understand the effect of desired membrane properties on the signaling networks, leading to synthesis of certain lipids that are sensitive to subtle structural alterations [221].

Finally, we hope to reiterate that despite the challenges still be addressed, lipids present a highly promising and detectable family of molecules for MSI studies, both for tissue classification/diagnostics (i.e., based on lipid profile) and for understanding the underlying biochemical processes occurring throughout biology. 


\section{Chapter 3 ULTRA-High MASS RESOLVING POWER, MASS ACCURACY, AND DYNAMIC RANGE MALDI MASS SPECTROMETRY IMAGING BY 21-TESLA FT-ICR MS}

\footnotetext{
A.P. Bowman ${ }^{1}$, Greg T. Blakney, Christopher L. Hendrickson ${ }^{2,3}$, S.R. Ellis ${ }^{1}$, R.M.A Heeren $^{1 *}$ and Donald F. Smith ${ }^{2 *}$

${ }^{1}$ Maastricht MultiModal Molecular Imaging (M4I) Institute, Division of Imaging Mass Spectrometry (IMS), Maastricht University, Universiteitssingel 50, Maastricht 6629ER, The Netherlands

${ }^{2}$ National High Magnetic Field Laboratory, Florida State University, 1800 East Paul Dirac Drive, Tallahassee, Florida 32310-4005, USA

${ }^{3}$ Department of Chemistry and Biochemistry, Florida State University, 95 Chieftain Way, Tallahassee, Florida 32306, USA
} 


\section{ABSTRACT}

Detailed characterization of complex biological surfaces by matrix-assisted laser desorption/ionization (MALDI) mass spectrometry imaging (MSI) requires instrumentation that is capable of high mass resolving power, mass accuracy, and dynamic range. Fourier transform ion cyclotron resonance mass spectrometry (FTICR MS) offers the highest mass spectral performance for MALDI MSI experiments, and often reveals molecular features that are unresolved on lower performance instrumentation. Higher magnetic field strength improves all performance characteristics of FT-ICR; mass resolving power improves linearly, while mass accuracy and dynamic range improve quadratically with magnetic field strength. Here, MALDI MSI at $21 \mathrm{~T}$ is demonstrated for the first time: mass resolving power in excess of 1,600,000 (at $\mathrm{m} / \mathrm{z} 400$ ), root-mean-square mass measurement accuracy below $100 \mathrm{ppb}$, and dynamic range per pixel over 500:1 were obtained from the direct analysis of biological tissue sections. Molecular features with $\mathrm{m} / \mathrm{z}$ differences as small as $1.79 \mathrm{mDa}$ were resolved and identified with high mass accuracy. These features allow for the separation and identification of lipids to the underlying structures of tissues. The unique molecular detail, accuracy, sensitivity, and dynamic range combined in a 21T MALDI FT-ICR MSI experiment enable researchers to visualize molecular structures in complex tissues that have remained hidden until now. The instrument described allows for future innovative, such as high-end studies to unravel the complexity of biological, geological, and engineered organic material surfaces with an unsurpassed detail.

\section{INTRODUCTION}

Mass spectrometry imaging (MSI) has proven to be versatile tool, finding applications in a variety of fields including diseased tissue classification[222-225], bacterial infections and resistance[199, 226], and drug metabolism[227, 228]. The main strength of MSI is the ability to simultaneously reveal the spatial distributions of multiple molecules in a single experiment from complex biological materials, typically tissue sections[229]. However, the chemically complex samples typically analysed bring challenges associated with the mass resolution and unambgious assignment of the many different molecules detected. Due to this complexity, many signals are often unresolved from isobaric ions, meaning generated ion images are 
not reflective one unique molecule. This is a major hindrance to studying the biochemical changes within tissues.

The most popular approach to begin addressing this complexity is the coupling of high mass resolving power and high mass accuracy analyzers with MSI ion sources, most commonly matrix-assisted laser desorption/ionization (MALDI)[230]. This coupling allows mass resolution of many isobaric ion species, as well as direct assignment of elemental composition, thereby providing insight into the specific identities of the detected molecules. For lipids, arguably the most widespread analyte class studies with MSI[210, 231-233], high mass resolving power and accuracy can facilitate true identification of sum-composition formula (i.e., lipid class and the combined number of carbons and double bonds across both fatty acyl chains), while separation of many isobaric species. In comparison to other biological molecules, resolving lipid complexity is further complicated by their relatively narrow mass range, with the majority of signals observed between $\mathrm{m} / \mathrm{z}$ 700-900[234]. Lipids can further be observed as multiple adducts (e.g., addition of $\mathrm{H}^{+}, \mathrm{Na}^{+}, \mathrm{K}^{+}, \mathrm{OAc}^{-}, \mathrm{Cl}^{-}$, or loss of $\mathrm{H}^{+}$) and are entangled with isotopes and other isobaric species, resulting in highly complex mass spectra that cannot be resolved with conventional high mass resolving power (e.g., $\leq 150,000 @ m / z$ 750)[234]. Thus, to unravel the spatial distributions of unique sum-composition lipid species that can have dramatically different biological functions, improvements in the achievable mass resolving of MSI technology is needed.

Fourier transform mass spectrometers; Fourier transform ion cyclotron resonance (FT-ICR) or orbital trapping (i.e., Orbitrap) offer higher mass resolving power and mass accuracy than other types of mass spectrometers (e.g., time-offlight and ion trap). FT-ICR mass spectrometers provide the highest mass resolving power and mass accuracy of any mass analyzer, with up to parts-per-billion (ppb) mass accuracy, high dynamic range, and mass resolving power values greater than $1,000,000$ in routine analyses[235-237]. Mass resolution and sensitivity in FT-ICR instrumentation can also be improved by the use of absorption mode processing[237, 238], although this has not yet been widely exploited for MSI applications[236]. In a proof-of-principle study, absorption mode MALDI FT-ICR MSI on a 9.4 T system provided mass resolving powers in excess of 250,000 for lipid species observed from mouse brain tissue[237, 239]. Several studies have shown similar high mass resolution on Orbitrap systems[217, 240, 241], though additional difficulties introduced in imaging systems typically report lower overall 
mass resolution[242-244]. High mass resolution is necessary to distinguish both nominally isobaric lipids, where common mass differences of less than 10 $\mathrm{mDa}$ [234] occur, as well as isotopic interferences, where mass differences less than $3 \mathrm{mDa}$ occur. While many lower field FT-ICR and Orbitrap instruments can distinguish the more common isobaric interferences, they are typically incapable of resolving mass differences less than $3 \mathrm{mDa}$ [245-247]. More recently, desorptionelectrospray ionization-MSI using a 7T FT-ICR system combined with absorption mode processing and external acquisition electronics demonstrated resolving powers up to $1,000,000$ for lipid species[236]. However, the number of ions had to be reduced to avoid space-charge and peak coalescence effects, which reduced the dynamic range by two orders of magnitude, and the $\mathrm{m} / \mathrm{z}$ range was truncated $(\mathrm{m} / \mathrm{z}$ 765-832). Higher magnetic field strength mitigates these problems, and enables larger ion populations to be analyzed, for high dynamic range broadband spectra at high mass resolution. The method described for DESI at $7 \mathrm{~T}$ helps overcome a key challenge in FT-ICR MSI by increasing the transient length while minimizing acquisition overhead, helping to balance the desired mass resolution with practical acquisition times for experiments that typically involve acquisitions of tens of thousands of spectra. These practical acquisition times are paramount within MALDI imaging, where the use of volatile matrices limits how long any single experiment can be performed before the matrix sublimes from the sample.

Outside of the improvements offered by absorption mode data processing, analysis times can be reduced by increasing the strength of the magnet used for FTICR. Mass resolution increases linearly with magnetic field strength[248], allowing for decreases in transient length without sacrificing resolving power. In the context of typically long MSI acquisition times, this improvement can reduce experimental times by several hours, a significant increase in throughput. Multiple frequency detection promises an increase in mass resolving power that scales linearly with the frequency order multiple[249]. However, to date this technique has not been applied to mass spectrometry imaging, though significant progress has been shown in ESI-based methods, which have reported mass resolving power of more than $10,000,000$ in the lipid range[235, 240,250].

The key parameters of FT-ICR that vary with magnetic field strength (dynamic range, mass accuracy, and ion-number induced frequency fluctuations) are especially important in MSI, due to the changes in ion yield depending on tissue type[251], as well as a lack of control (e.g., via automatic gain control) over the 
number of ions entering the analyzer cell at each pixel. Further, the rich information available within the lipid range sees an enormous benefit from higher magnetic fields, in part from the biological dynamic range of lipids, but also from the number of nominally isobaric peaks possible in biological tissues. The advantage of increased mass resolution is obvious, but the improvement to mass accuracy and dynamic range can be crucial. High mass accuracy over long analysis times is important to generate highly accurate MSI images, as any drift in mass across an experiment would necessitate either pixel-to-pixel correction for this drift, or wider mass selection windows for image generation to encapsulate the ion as its apparent $\mathrm{m} / \mathrm{z}$ shifts over time. High-field FT-ICR mass spectrometers offer external mass calibrations of less than 0.2 parts per million (ppm)[252], and internal calibration less than $0.1 \mathrm{ppm}$ [253]. High dynamic range is a key performance metric for MSI, given the wide dynamic range of lipid concentrations[254], and differences in the ionization efficiencies of these biomolecules. Increased dynamic range is important to distinguish low abundance species while still detecting highly abundant lipids without distortion in relative ion abundances. The higher the magnetic field of an FT-ICR, the less susceptible it is to ion-number induced frequency shifts, which can hinder identification of peaks and complicate calibration of datasets, as has been described previously[255-257].

Within the field of lipidomics, both shotgun and LC-MS based methodologies have achieved mass resolution in the lipid range greater than 100,000 along with sub-ppm mass accuracy, enabling assignment of 200-500 lipids in a single experiment[258-260]. Due to the increased fluctuations in signal intensity inherent to MSI, progress towards such endeavors is slower, success has been shown in a variety of FT based instruments with numerous ionization techniques, including Liquid Extraction Surface Analysis[261], MALDI[262], DESI[236], and LAESI[239]. LAESI was performed on a 21 T FT-ICR mass spectrometer which separated the isotopic fine structure of nominally overlapping metabolites of plant leaves, which improved identification by utilizing multiple peaks per metabolite in the identification process. Additionally, the experimental time frame for the $21 \mathrm{~T}$ is significantly reduced compared to other instruments with similar mass resolution, without sacrificing either signal magnitude or mass range, as has been attempted with lower-field instruments[236, 239, 253].

In this work, we evaluate for the first time the performance of MALDI MSI combined with 21 T FT-ICR MS for biological tissue imaging, as well as the use 
of automated annotation to begin exploring the highly complex information available from such experiments. In particular, we demonstrate (i) the combined higher mass resolving power and mass accuracy with the stability of these parameters across long MSI experiments; (ii) increased biochemical information obtained during MALDI MSI facilitated by the high mass resolving power and mass accuracy; (iii) single-pixel dynamic range exceeding 500:1, which enables imaging and identification of very low abundance ions; (iv) automated analytical tools to identify potentially hundreds of lipids utilizing thousands of peaks. Combined, this work demonstrates the high potential of MALDI MSI and 21 T FTICR for studying localized biomolecular processes within tissues and their diseaseinduced alterations.

\section{METHODS}

\section{MATERIALS}

Methanol (LC-MS grade), ethanol (LC-MS grade), xylene (LC-MS grade), water (LC-MS grade), anhydrous chloroform ( $\geq 99.9 \%$ purity), and crystalline norharmane (9H- $\beta$-carboline) were purchased from Sigma Aldrich (Zwijndrecht, The Netherlands) and used without further purification. Indium tin oxide (ITO)coated glass slides were purchased from Delta Technologies (Loveland, USA).

\section{BIOLOGICAL SAMPLES}

Healthy rat brain was obtained from Maastricht University in accordance with protocols approved by the Animal Care and Use Committee under Animal Experiment Committee (DEC) number 2016-006 AVD107002016720. 4 transverse rat brain segments $\left(12-\mu \mathrm{m}\right.$ thick) were sectioned with a cryo-microtome at $-20{ }^{\circ} \mathrm{C}$ and thaw-mounted on ITO-coated glass slides. Some distortion of the tissue sections occurred during the mounting process.

SAMPLE PREPARATION

Norharmane matrix $(7 \mathrm{mg} / \mathrm{mL})$ in $\mathrm{CHCl}_{3}: \mathrm{MeOH}(2: 1 \mathrm{v} / \mathrm{v})$ was applied to the tissue with a TM-Sprayer (HTX Technologies, City, USA). Spray conditions were as follows: flow rate, $0.12 \mathrm{~mL} / \mathrm{min} ; \mathrm{N}_{2}$ pressure, $10 \mathrm{psi} ; \mathrm{N}_{2}$ temperature, $30{ }^{\circ} \mathrm{C}$; spray-head velocity, $1,200 \mathrm{~mm} / \mathrm{min}$; track spacing, $3 \mathrm{~mm}$; number of layers, 15; drying time between layers, $30 \mathrm{~s}$. 


\section{INSTRUMENTATION}

All MSI experiments were performed on a hybrid linear ion trap $21 \mathrm{~T} \mathrm{FT-}$ ICR mass spectrometer at the National High Magnetic Field Laboratory (NHMFL) at Florida State University (Tallahassee, USA). A Velos Pro linear ion trap (Thermo Scientific, San Jose, USA) was combined with NHMFL-designed external linear quadrupole ion trap, quadrupole ion transfer optics, and a novel dynamically harmonized ICR cell, which is operated at 7.5-V trapping potential[252]. Briefly, the cell uses $120^{\circ}$ cell segments for ion excitation and detection, for improved excitation electric field, detection sensitivity, and reduced third harmonic signals[263, 264].

The commercial ion source and stacked ring ion guide were replaced with an elevated-pressure MALDI ion source incorporating a dual-ion funnel interface (Spectroglyph LLC, Kennewick, USA) as has been described previously[265]. Voltages within the funnels were $625 \mathrm{kHz}, 150 \mathrm{~V}$ peak-to-peak (first, high-pressure ion funnel), and 1.2 MHz, $90 \mathrm{~V}$ peak-to-peak (second, low-pressure ion funnel). An electric field gradient of $\sim 10 \mathrm{Vcm}^{-1}$ was maintained within the dual-funnel system, with a gradient of $100 \mathrm{Vcm}^{-1}$ between the sample and the funnel inlet. The system was equipped with a Q-switched, frequency-tripled Nd:YLF laser emitting 349-nm light (Explorer One, Spectra Physics, Mountain View, USA). The laser was operated at a repetition rate of $1 \mathrm{kHz}$ and pulse energy of $\sim 1.2 \mu \mathrm{J}$. Pressure within the ion source was set to 10 mbar in the first ion funnel, and 2 mbar in the second ion funnel. MALDI stage motion was synchronized with ion accumulation using the Velos trigger signal indicating commencement of the ion trap injection event, as previously described.[265] The mass spectrometer was operated with an ion injection time of $250 \mathrm{~ms}$ and automatic gain control (AGC) was turned off. A transient duration of $3.1 \mathrm{~s}$ was used for ultra-high mass resolving power analyses, resulting in a total time of $4 \mathrm{~s}$ per pixel. Spectra were obtained in both positive and negative mode, at $100 \mu \mathrm{m}$ spatial resolution. Total number of pixels per brain section were approximately 22,000, and 24 hours of experimental time. A Predator data station was used for ion excitation and detection[266].

\section{DATA PROCESSING AND ANALYSIS}

Absorption mode mass spectra transients were phased-corrected[267], and peaks with a signal magnitude greater than 6 times the standard deviation of the 
baseline root-mean-square (RMS) noise were exported to peak lists. Mass calibration was performed on known lipid species, with a wide range of spectral intensities ([PC 34:1 + K $]^{+},[\mathrm{SM} \mathrm{34:1;2+} \mathrm{H}]^{+},\left[\mathrm{PE} 36: 4{ }^{13} \mathrm{C}+\mathrm{H}\right]^{+},[\mathrm{PC} 32: 0+\mathrm{Na}]^{+}$, $[\mathrm{PC} 34: 1+\mathrm{H}]^{+},[\mathrm{PC} 38: 4+\mathrm{Na}]^{+}$, and $[\mathrm{PC} 38: 4+\mathrm{K}]^{+}$) and the data were converted to imzML format using in-house MATLAB routines, msconvert from the ProteoWizard software suite (version 3.0.11537)[268], and imzMLConverter version 1.3[269]. The ALEX ${ }^{123}$ software package was used for sum-composition lipid identification at a search tolerance of $1 \mathrm{mDa}[259,270]$. All phospholipid classes were chosen, as were sphingolipids and glycerolipids, with chain-lengths of 14 carbons or greater. Adducts were limited to $\mathrm{H}^{+}, \mathrm{Na}^{+}$, and $\mathrm{K}^{+}$, and negative mode was restricted here to simple loss of $\mathrm{H}^{+}$. Images generated are normalized to the total ion current per pixel (TIC).

RESULTS AND DISCUSSION 


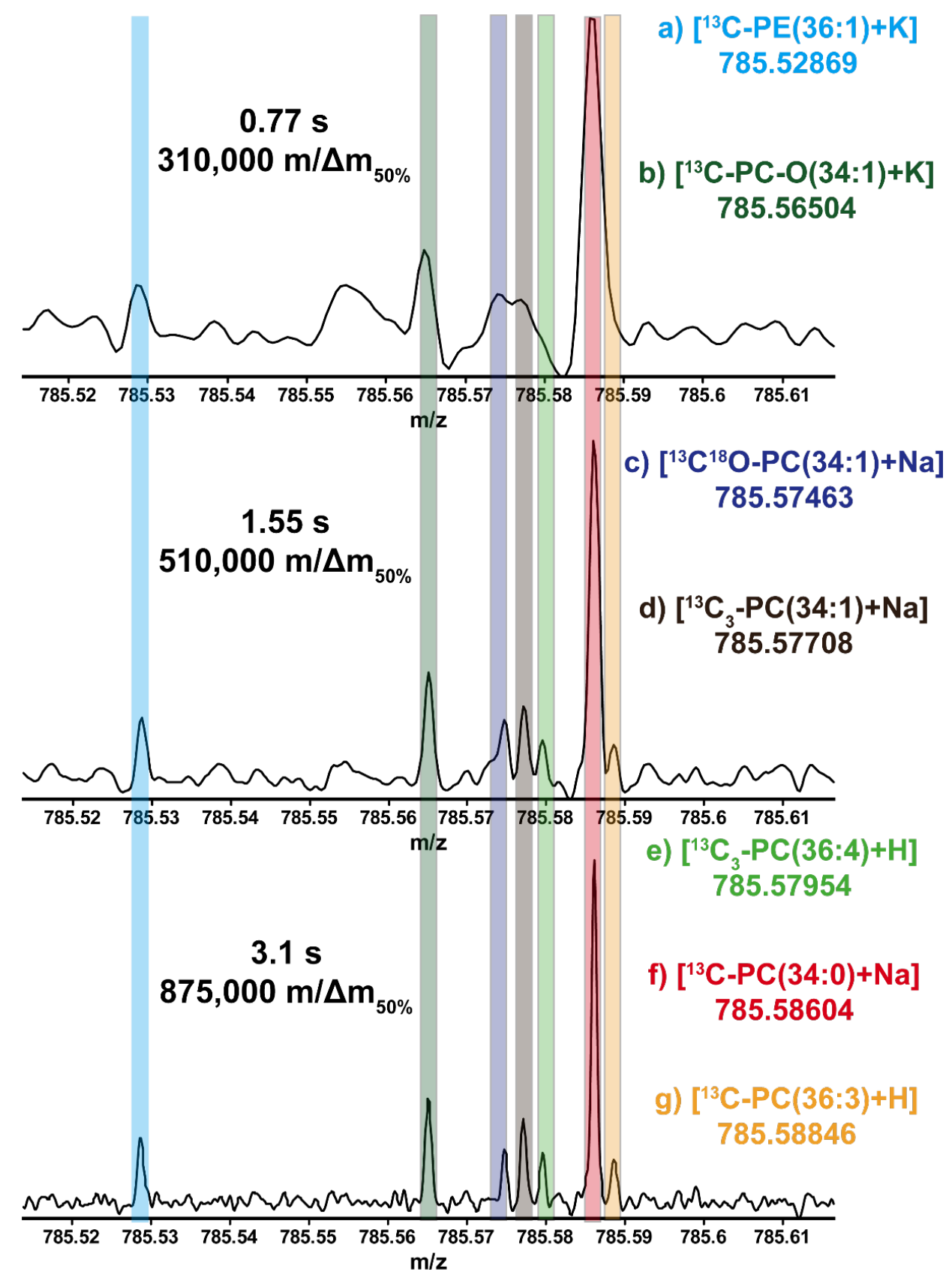

Figure 3.1. Mass resolution and sensitivity improve with longer transient length. Within a $100 \mathrm{mDa}$ mass range, seven different peaks are detected, which belong to six different lipid species. Of these, five are unresolved at 0.77 $\mathrm{s}$. While distinguishable at $1.55 \mathrm{~s}$, all seven peaks are fully resolved only at $3.1 \mathrm{~s}$ transient. These seven peaks correspond to the isotopologues of the monoisotopic species, typically the $13 \mathrm{C}$ ion, as in a), b), f), and g). Other species are also present, corresponding to the $13 \mathrm{C} 3$ isotopologue, as in $\mathrm{d}$ ) and e). The $18 \mathrm{O} 13 \mathrm{C}$ isotopologue of $[\mathrm{PC}(34: 1)+\mathrm{Na}]+$ is also resolved (c) from the $13 \mathrm{C} 3$ isotopologue of the same parent species 


\section{HIGH MASS RESOLVING POWER.}

To assess the benefits of performing 21 T MALDI MSI in terms of mass resolving power, we analyzed rat brain sections in both positive and negative ion mode using different transient acquisition times. Figure 3.1 shows the achieved mass resolving power in the positive-ion mode using $0.76,1.55$ and $3.1 \mathrm{~s}$ transients within the $\mathrm{m} / \mathrm{z}$ range 785.52-785.6. Increasing mass resolution shows increasing spectral complexity, as 5 peaks are resolved from what first appears to be only 2 , with 2 additional peaks within $100 \mathrm{mDa}$ which were sufficiently distinct to be identified at all transient lengths. We annotated the 7 peaks within this region as belonging to 6 different species of lipids: $[\mathrm{PE}(36: 1)+\mathrm{K}]^{+}$, [PC-O $\left.(34: 1)+\mathrm{K}\right]^{+}$, $[\mathrm{PC}(34: 1)+\mathrm{Na}]^{+},[\mathrm{PC}(36: 4)+\mathrm{H}]^{+},[\mathrm{PC}(34: 0)+\mathrm{Na}]^{+}$, and $[\mathrm{PC}(36: 3)+\mathrm{H}]^{+}$. Of these, 4 are the ${ }^{13} \mathrm{C}_{1}$ isotope: $\left.\quad\left(\left[\mathrm{PE}(36: 1)+{ }^{13} \mathrm{C}+\mathrm{K}\right]\right]^{+}, \quad\left[\mathrm{PC}-\mathrm{O}(34: 1)+{ }^{13} \mathrm{C}+\mathrm{K}\right]\right]^{+}$, $\left.\left[\mathrm{PC}(34: 0)+{ }^{13} \mathrm{C}+\mathrm{Na}\right]\right]^{+}$, and $\left.\left[\mathrm{PC}(36: 3)+{ }^{13} \mathrm{C}+\mathrm{H}\right]+\right), 2$ are the ${ }^{13} \mathrm{C}_{3}$ isotope $([\mathrm{PC}(34: 1)+$ $\left.{ }^{13} \mathrm{C}_{3}+\mathrm{Na}\right]^{+}$and $\left.\left[\mathrm{PC}(36: 4)+{ }^{13} \mathrm{C}_{3}+\mathrm{H}\right]^{+}\right)$, and the final peak is the ${ }^{13} \mathrm{C}^{18} \mathrm{O}$ isotope $\left(\left[\mathrm{PC}(34: 1)+{ }^{13} \mathrm{C}^{18} \mathrm{O}+\mathrm{Na}\right]^{+}\right)$. These peaks show mass accuracy errors between -50 and 13 parts-per-billion (ppb). Additionally, isotope ratios in the summed average spectra deviate $<15 \%$ from theoretical in these 7 peaks (Figure 3.2), offering additional certainty in that correct sum-composition identification has been made, as well that there are no convoluted peaks being presented as a single peak. Deviation from the expected 2-fold improvement in mass resolving power upon doubling of the transient duration is due to known collisional damping during the detection event[252]. Current work focuses on a solution to limit transmission of the neutral buffer gas in the external accumulation multipole to the ultra-high vacuum region. Recently, a mass resolving power of $\sim 600,000$ (at $\mathrm{m} / \mathrm{z} 760$ ) for MALDI MSI on a $15 \mathrm{~T}$ FT-ICR MS (the highest commercially magnetic field available for FT-ICR) was reported. This value also deviates from the theoretical mass resolving power for a $5.2 \mathrm{~s}$ transient (magnitude mode), which is $\sim 788,000[271]$. 


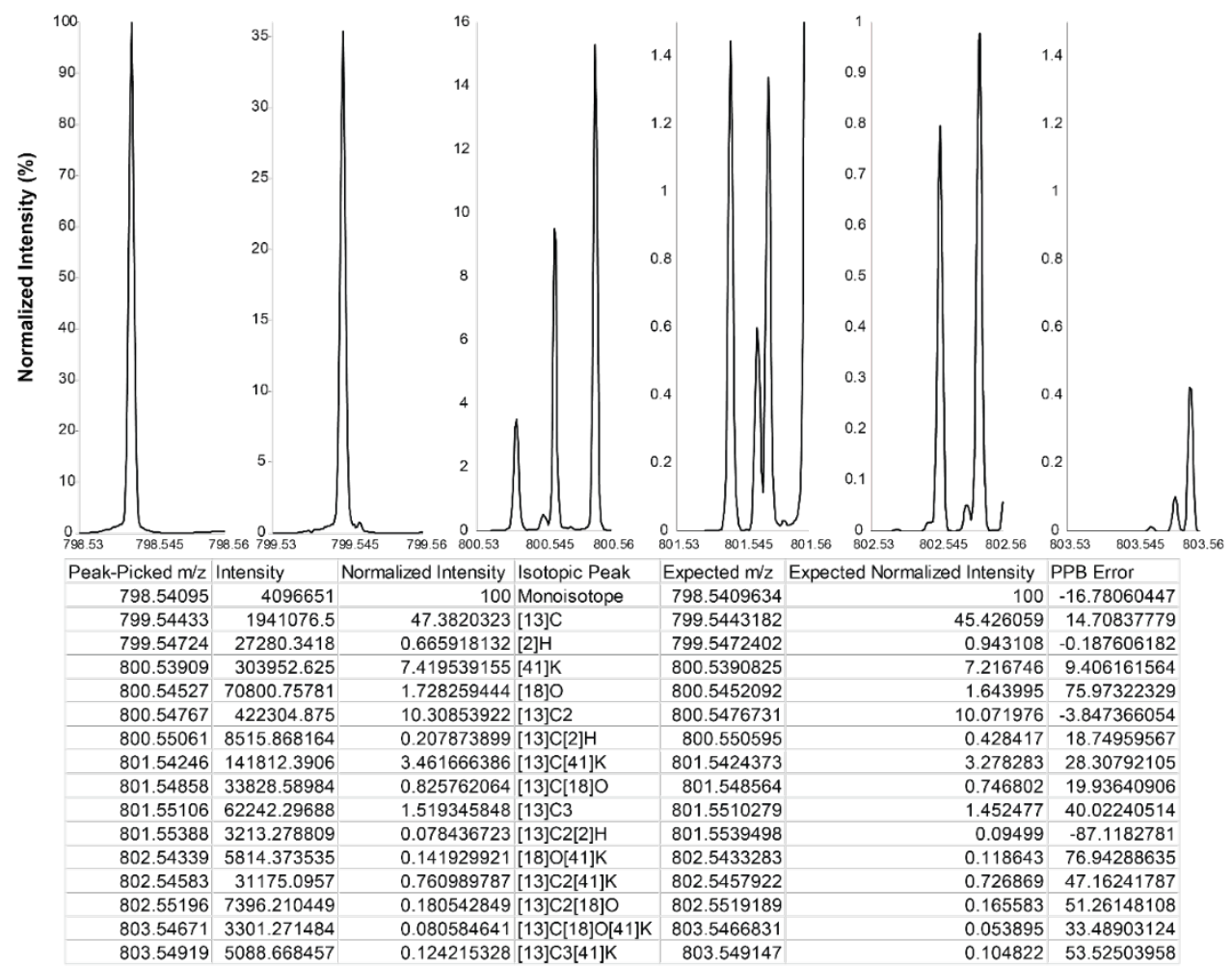

Figure 3.2 Single scan chromatograms expanded on the isotopes of $[\mathrm{PC}(34: 1)+\mathrm{K}]^{+}$. Also listed are the identified isotopic peaks from the averaged spectra, as well as their comparisons to theoretical.

To further assess the utility of the $21 \mathrm{~T}$, we analyzed the dataset for peaks with close neighbors (here defined as $<10 \mathrm{mDa}$ ). We extracted the $3.1 \mathrm{~s}$ transient from a single pixel (number 10,000) as a representative spectrum from each dataset. In positive-ion mode, a difference of $0.0024 \mathrm{Da}(2.4 \mathrm{mDa})$ at $\mathrm{m} / \mathrm{z} 810$ was present (Figure 3.3a), representing the difference between $\mathrm{Na}_{1} \mathrm{H}_{1}$ versus $\mathrm{C}_{2}$ (the addition of two carbon atoms and three double bonds to the lipid fatty acid chains) which requires a mass resolving power $\left(\mathrm{m} / \Delta \mathrm{m}_{50 \%}\right)$ of 337,000 at $\mathrm{m} / \mathrm{z} 810$ to resolve. These two ions were well resolved, and lipid identities were assigned $[\mathrm{PC}(36: 1)+\mathrm{Na}]^{+}$and $[\mathrm{PC}(38: 4+\mathrm{H})]^{+}$with high confidence (100 ppb, see discussion below). Each species had very different spatial distributions, with the former $\left([\mathrm{PC}(36: 1)+\mathrm{Na}]^{+}\right)$being relatively evenly distributed (Figure $3.3 \mathrm{~b}$ ), while the latter $\left([\mathrm{PC}(38: 4+\mathrm{H})]^{+}\right)$had higher abundance in the lateral ventricle (Figure 3.3c). The higher abundance of $[\mathrm{PC}(38: 4)+\mathrm{Na}]^{+}$in the ventricles matches with its role as a pro-inflammatory cytokine[272]. Interestingly, such a small mass difference was not uncommon, with 
a mass difference of $2.4 \mathrm{mDa}$ observed over 190 times in any single pixel spectrum, and more than 1,000,000 times over a single MSI experiment (Figure 3.3d). Without sufficient mass resolving power, any one of the images of these $\sim 190$ pairs of closely spaced ions could yield incorrect assignments and yield a summed spatial distribution reflective of neither individual species. A variety of other recurrent mass differences can be detected in the single spectra, ranging from 1-10 $\mathrm{mDa}$, including isotopic patterns (e.g., ${ }^{13} \mathrm{C}_{2} \mathrm{vs} \mathrm{H}_{2}$ is a difference of $8.94 \mathrm{mDa}$ ). The change in ${ }^{13} \mathrm{C}_{2} \mathrm{vs} \mathrm{H}_{2}$ is an important one, as this denotes the possible overlap for species that differ by a single double bond (i.e., as $\mathrm{PC}(34: 1)$ to $\mathrm{PC}(34: 0)$ ). Single unsaturation changes have been shown to be important in various types of disease states, including cancers[194, 196] and multiple sclerosis[273], and so the ability to resolve such fine mass differences opens the door to studying the precise roles of the subtle changes in lipid structure throughout tissues. 
a) Positive-ion mode

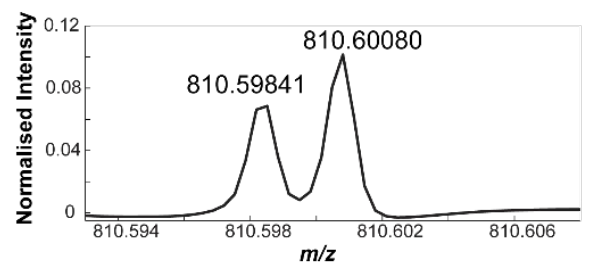

b) $\mathrm{m} / \mathrm{z} 810.59841$

$[\mathrm{PC}(36: 1)+\mathrm{Na}]^{+}$

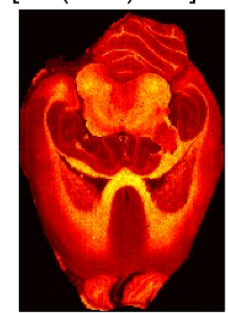

d) Positive-ion mode

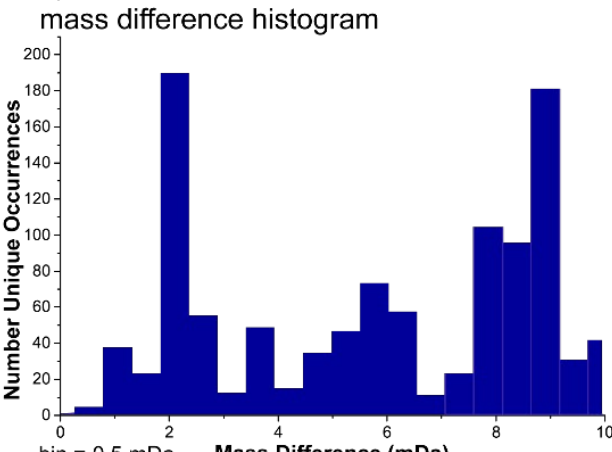

C) $\mathrm{m} / \mathrm{z} 810.60080$
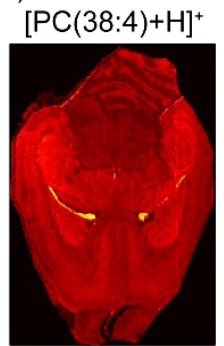

e) Negative-ion mode

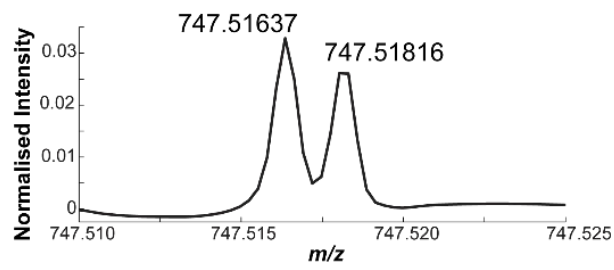

f) $\mathrm{m} / \mathrm{z} 747.51637$

$\left[\mathrm{PE}(\mathrm{O}-38: 7)^{13} \mathrm{C}_{1}-\mathrm{H}\right]^{-}$

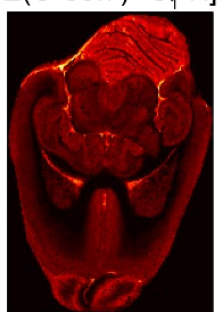

g) $m / z 747.51816$

h) Negative-ion mode

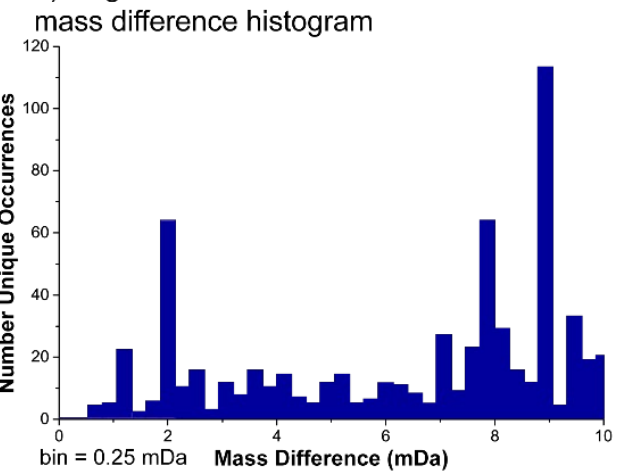

Figure 3.3. Representative images of close mass differences in negative and positive mode, from a single, scan. Images are total ion current normalized. Positive mode lipid spectra have a significant number of mass differences of $2.4 \mathrm{mDa}$ (a), representing the difference between ${ }^{12} \mathrm{C}_{2}$ and ${ }^{23} \mathrm{Na}^{1} \mathrm{H} .2 .4 \mathrm{mDa}$ differences are resolved at better than baseline separation, and show significantly different distributions within brain tissue (b and c). There are nearly 200 such differences in the averaged spectra, shown in $0.5 \mathrm{mDa}$ bins (d). Similarly, negative mode spectra have $1.79 \mathrm{mDa}$ mass differences (e). These $1.79 \mathrm{mDa}$ differences are resolved to better than full-width halfmaximum, differentiated well enough to distinguish them in brain tissue ( $\mathrm{fand} g$ ). The of $1.79 \mathrm{mDa}$ mass difference is relatively uncommon in negative mode, but mass differences of $10 \mathrm{mDa}$ or less occur approximately 500 times in the averaged spectra, shown in $0.25 \mathrm{mDa}$ bins (h).

Using the same experimental design in the negative-ion mode, additional small mass differences could be resolved. For example, a mass difference of $0.00179 \mathrm{Da}(1.79 \mathrm{mDa})$ at $\mathrm{m} / \mathrm{z} 757.52$ was observed at 31 different masses. This corresponds the mass difference of $\mathrm{C}_{2} \mathrm{~N}_{1}{ }^{13} \mathrm{C}_{1}$ versus $\mathrm{H}_{3} \mathrm{O}_{3}$ (Figure 3.3e). While less common than the $\mathrm{NaH}$ vs. $\mathrm{C}_{2}$ split in positive mode, ether-linked phosphatidylethanolamine (PE) and PC lipids can have this difference from the 
phosphatidylglycerol (PG) class. These peaks were thus identified as phosphatidylethanolamine $\left[\mathrm{PE}(\mathrm{O}-38: 7)+{ }^{13} \mathrm{C}-\mathrm{H}\right]^{-}$and $\left.[\mathrm{PG}(34: 1)-\mathrm{H}]\right]^{-}$. This is the smallest mass difference observed in any MSI dataset to date. The PE is a ${ }^{13} \mathrm{C}$ containing nuclide of the monoisotopic PE lipid at $\mathrm{m} / \mathrm{z}$ 746.51300. PE and PG lipids are synthesized by different biological pathways and have different physiological function. PE lipids are $\sim 20 \%$ of all phospholipids, and are especially abundant in white matter of the cerebellum (Figure 3.3f)[274]. By contrast, PG lipids are associated with ATP-Binding Cassette 3, though what transport function is utilized is unknown[275]. The $1.79 \mathrm{mDa}$ mass difference occurred over 100,000 times in our MSI experiment, with 33 unique pairs detected in the total mass spectrum. As in the positive mode, the ${ }^{13} \mathrm{C}_{2}$ vs $\mathrm{H}_{2}$ difference occurs regularly, and has many of the same ramifications as discussed above.

DYNAMIC RANGE.

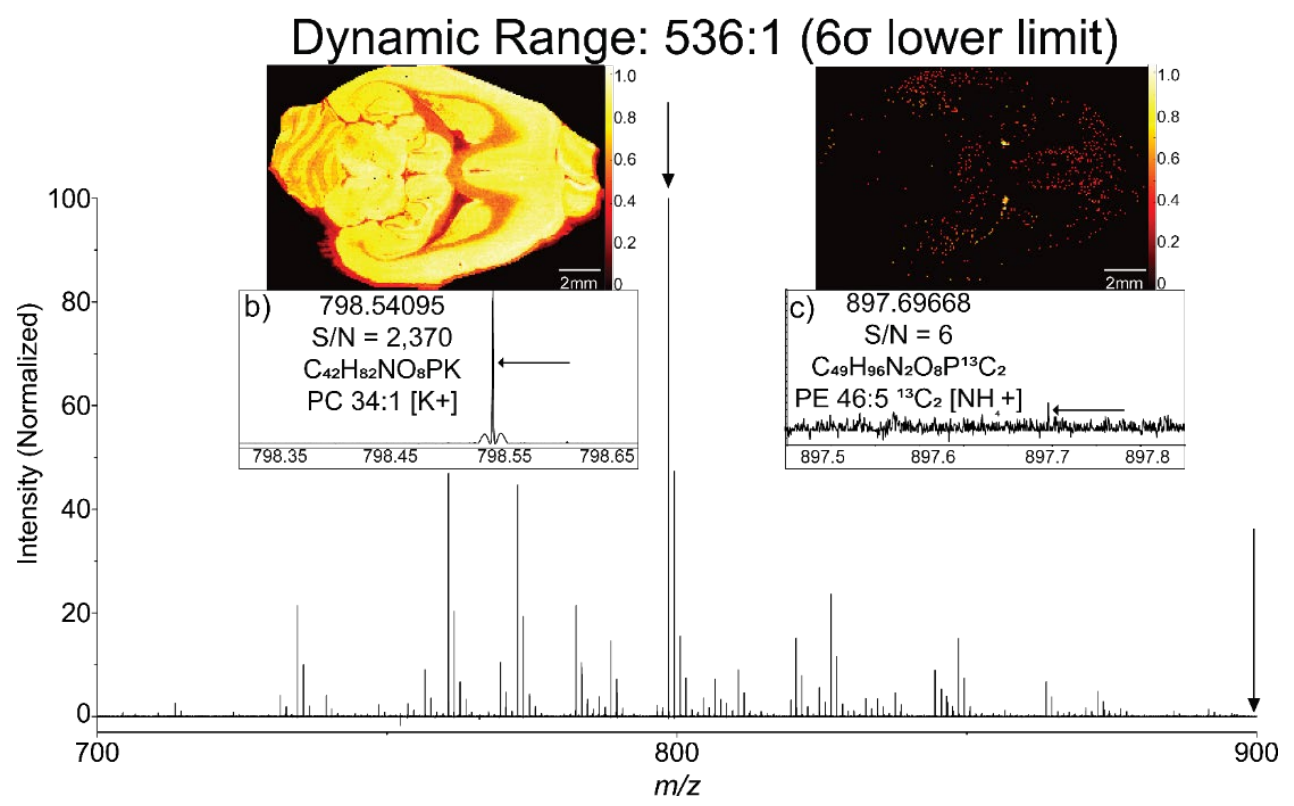

Figure 3.4. Single on-tissue mass spectrum illustrates high dynamic range per pixel. Peaks were picked at a threshold of six standard deviations above the baseline noise. Dynamic range in a single average pixel of at 536:1 is demonstrated here at pixel number 10,000, (a). Mass scale expanded segment around most abundant peak [PC $34: 1+\mathrm{K}]^{+}$(b). Further, peak at 798.5410 generates a bright image (b). One of the lowest $\mathrm{S} / \mathrm{N}$ peaks, the ${ }^{13} \mathrm{C}_{2}$

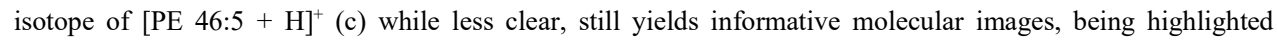
especially in the ventricles (images are TIC normalized). 
One of the most problematic complications in MSI is the low relative ionization efficiency from the surface, which combined with the wide range of analyte concentrations, places significant demands on the single scan dynamic range achievable in an MSI experiment. High sensitivity and dynamic range are thus necessary to detect low abundance and/or poorly ionized species without distorting the peak abundances obtained from high intensity signals. Figure 3.4a shows a single pixel mass spectrum of the lipid $\mathrm{m} / \mathrm{z}$ range from the positive-ion mode dataset (scan \#10,000), which has a dynamic range of 536:1 (expanded mass range spectrum shown in Figure 3.5).

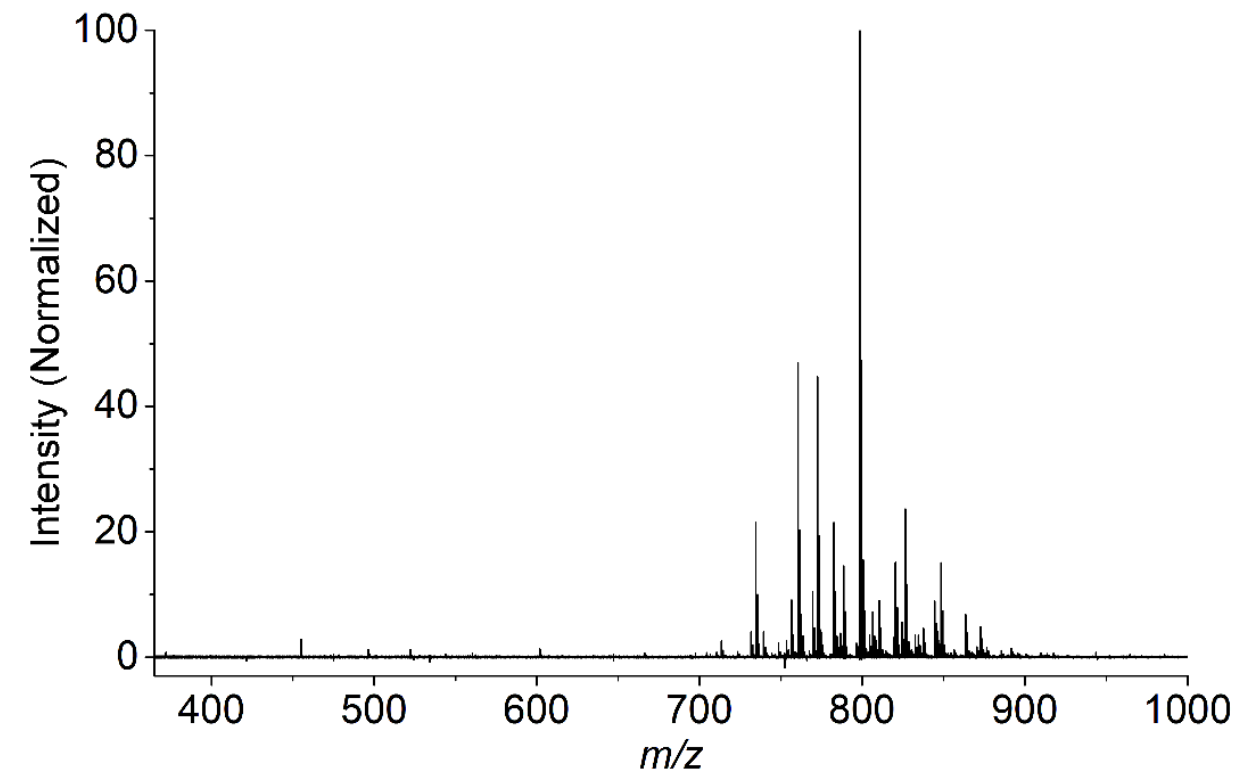

Figure 3.5 Expanded mass spectral range for scan $\# 10,000$, representing approximately half of the full mass range per pixel.

The dynamic range was calculated by dividing the signal magnitude of the base peak by the peak detection threshold of six standard deviations $(6 \sigma)$ above the baseline noise. As typically observed from brain tissue, the $[\mathrm{M}+\mathrm{K}]^{+}$ion of $\mathrm{PC}(34: 1)$ generated the highest signal magnitude, with a signal-to-noise=2,370:1 (Figure $3.4 \mathrm{~b}$; side lobe artifacts are a result of the absorption mode processing, and current work is focused on their removal). By contrast, rat brain tissue sections prepared from the same original organ a under the same conditions showed a signal-tonoise=336:1 on a Thermo Orbitrap Elite set at 240,000 resolving power (@ m/z 400) at the Maastricht MultiModal Molecular Imaging Institute. Using a peak 
detection threshold of $6 \sigma$ above the baseline noise, the lowest intensity signal was observed with a signal-to-noise $=6$ and corresponded to the $\left[\mathrm{PE}(46: 5)+{ }^{13} \mathrm{C} 2+\mathrm{H}\right]+$ (Figure 3.4c).

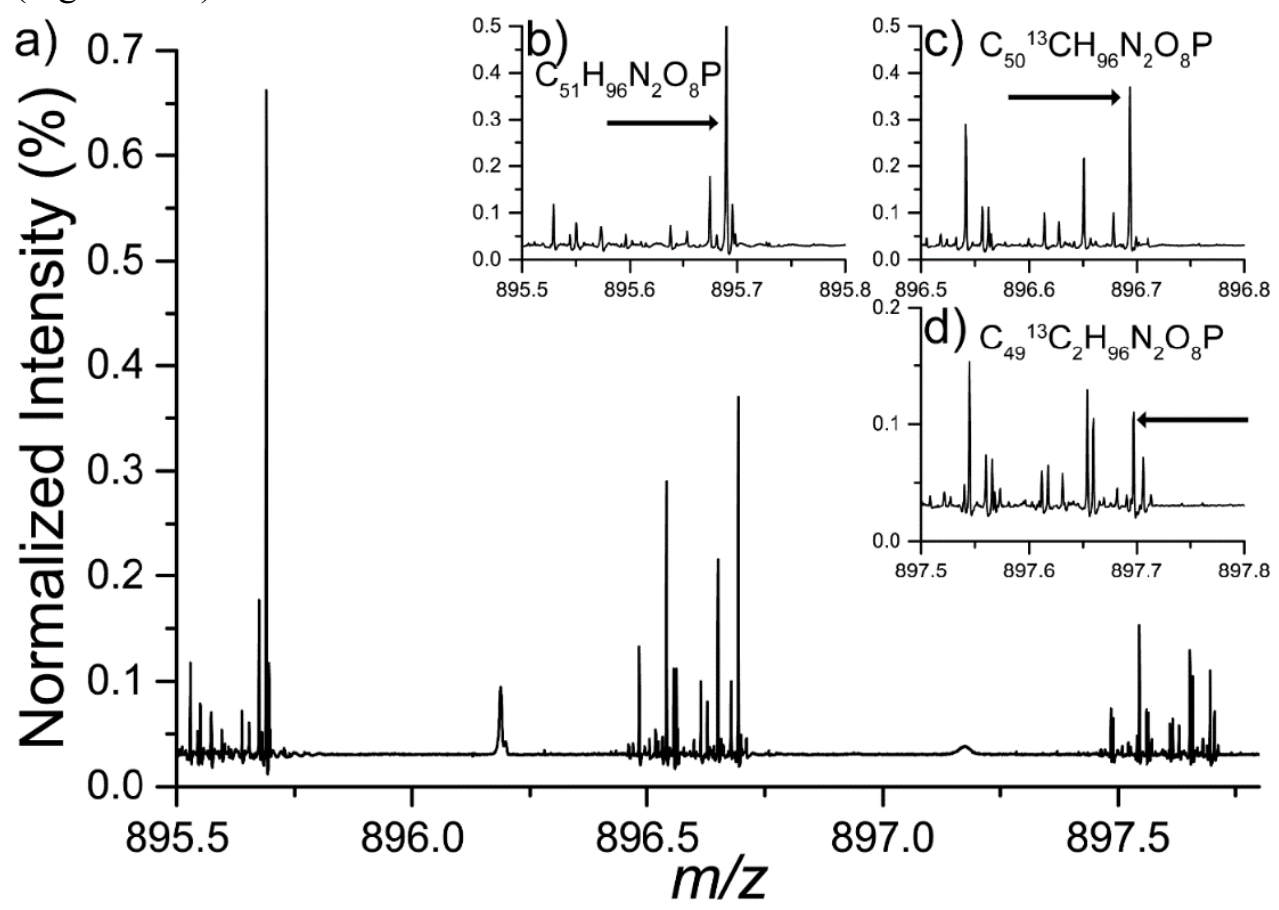

Figure 3.6. Single pixel spectra of $\left[\mathrm{PE}(46: 5)+\mathrm{NH}_{4}\right]^{+}$. Full spectra of all identifiable isotopes available in (a). Monoisotopic mass shown in (b), first ${ }^{13} \mathrm{C}$ isotope in (c), and ${ }^{13} \mathrm{C}_{2}$ isotope in (d).

Figure 3.6 shows the isotopic distribution for PE 46:5, where the ${ }^{13} \mathrm{C}_{2}$ containing nuclide can be identified at $\mathrm{M}+2$. 

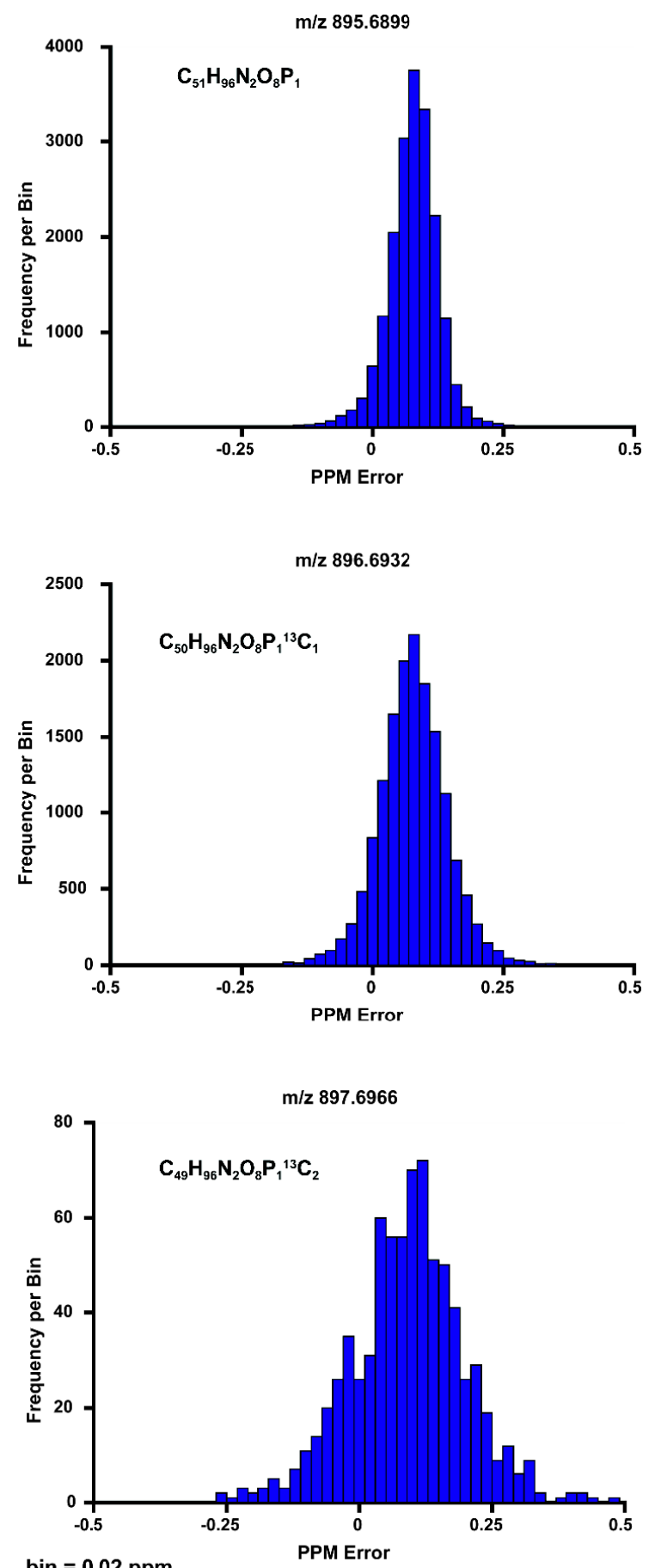

bin $=0.02 \mathrm{ppm}$

Figure 3.7. PPM error distribution of 3 most abundant isotopologues of $\left[\mathrm{PE}(46: 5)+\mathrm{NH}_{4}\right]^{+}$. 
Figure 3.7 shows ppm error distributions for the monoisotopic peak, $\mathrm{M}+1$ $\left({ }^{13} \mathrm{C}_{1}\right)$, and $\mathrm{M}+2\left({ }^{13} \mathrm{C}_{2}\right)$ which show good mass accuracy, despite the low $\mathrm{S} / \mathrm{N}$ of the $\mathrm{M}+2$ peak. Per pixel, the average dynamic range in positive ion mode was $438: 1$, with a maximum dynamic range of 2090:1 and a minimum of 60:1 (Figure 3.8). Negative-ion mode spectra had lower signal magnitude than positive mode, limiting the average dynamic range to $214: 1$, with a maximum of $849: 1$ and minimum of 30:1 (Figure 3.9).

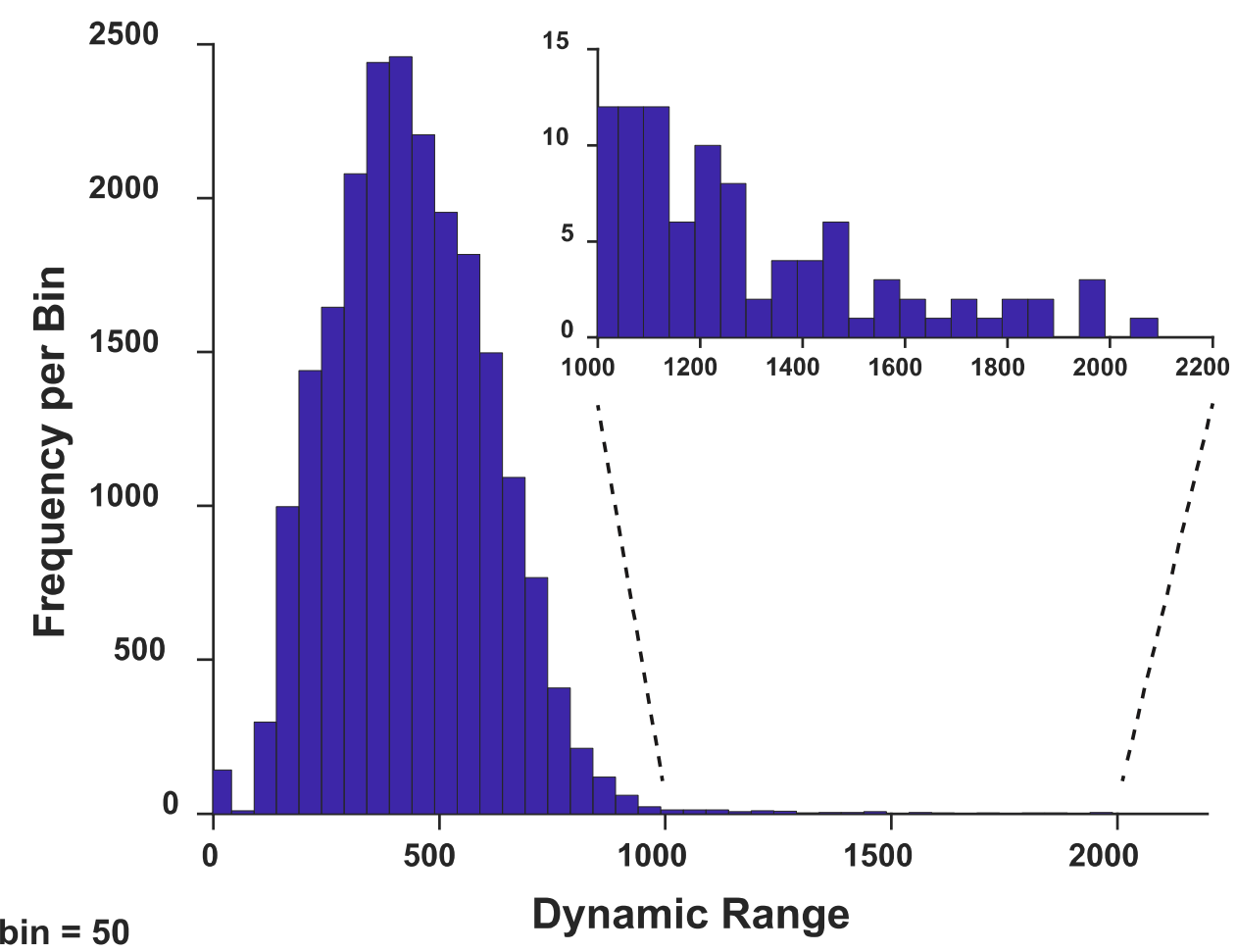

Figure 3.8. Histogram of dynamic range for positive-ion mode. Bin size $=50$. 


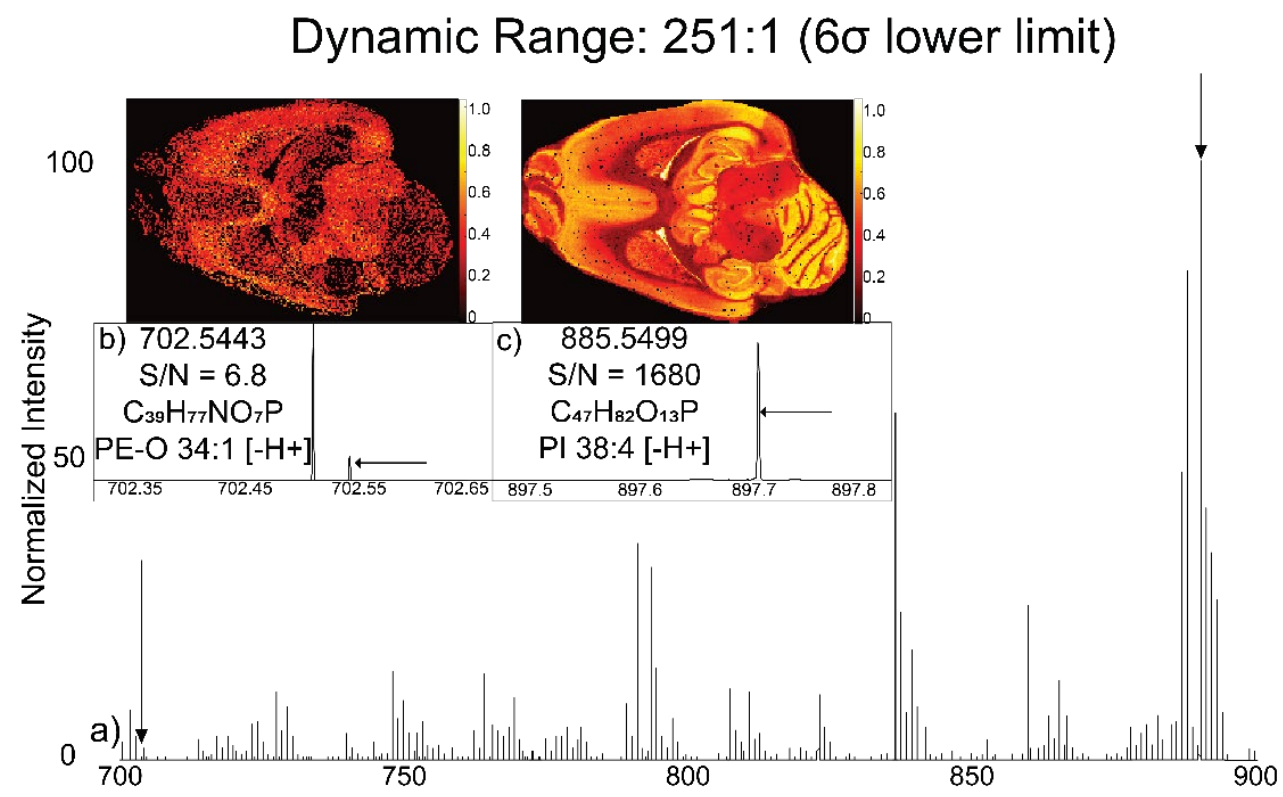

Figure 3.9. Single on-tissue mass spectrum of the negative ion-mode, demonstrating high dynamic range. Peaks were picked at a threshold of 6 standard deviations above the baseline noise. Dynamic range in a single average pixel is demonstrated here by pixel number 10,000, at 251:1 (a). The spectra is expanded to show the spectrum around the lowest peak, in this spectrum as [PE-O(34:1)-H]- (b). An expanded view around the most abundant peak [PI(38:4)-H]- (c).

\section{HIGH MASS ACCURACY.}

FT-ICR MSI at $21 \mathrm{~T}$ showed a root-mean-square (rms) mass measurement accuracy of $62.12 \mathrm{ppb}$ (Figure 3.10a), over 2-fold lower rms mass accuracy achieved on a $9 \mathrm{~T}$ instrument, which was limited to an rms of $158 \mathrm{ppb}$ [276]. The center of the distribution is centered near zero, and the low standard deviation indicates low $\mathrm{m} / \mathrm{z}$ fluctuation during the imaging experiment. 

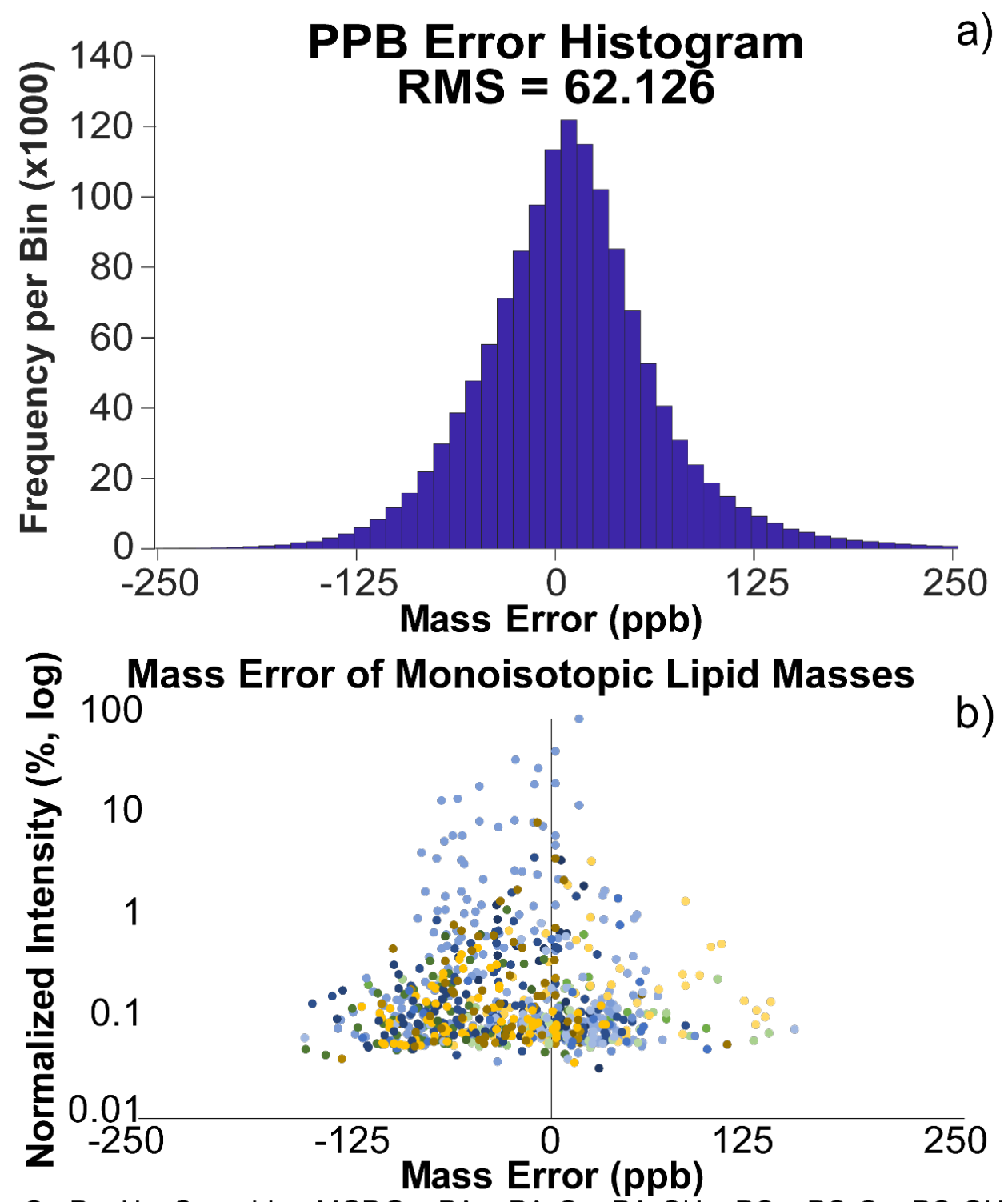

- CerP HexCer $\bullet \mathrm{LL} \bullet \mathrm{MGDG} \bullet \mathrm{PA} \bullet \mathrm{PA}-\mathrm{O} \bullet \mathrm{PA}-\mathrm{OH} \bullet \mathrm{PC} \bullet \mathrm{PC}-\mathrm{O} \bullet \mathrm{PC}-\mathrm{OH}$

- PE • PE-Cer P PE-O ค PE-OH • PI-Cer ค PS • PS-O • PS-OH • SM • TG Figure 3.10. Error histogram and average mass error of tentatively identified lipids after internal calibration. Measured mass error histogram of 139 phosphatidylcholine lipids; the rms error is $61.12 \mathrm{ppb}$. Bin size $=10 \mathrm{ppb}$. (a), Lipid identifications by class. A tolerance of $+/-250 \mathrm{ppb}$ results in 702 potential lipids identified within 150 ppb of their expected mass (b).

Figure 3.11 shows the measured $\mathrm{m} / \mathrm{z}$ variation for $[\mathrm{PC}(36: 1)+\mathrm{H}]^{+}(\mathrm{m} / \mathrm{z}=$ 788.61638, dotted red line indicates the exact $\mathrm{m} / \mathrm{z}$ ) over the imaging experiment, where the maximum $\mathrm{m} / \mathrm{z}$ deviation is 0.00018 , with a standard deviation of 0.00078 . 
Internal calibration was performed using 7 tentatively identified lipid masses $\left([\mathrm{PC}(34: 1)+\mathrm{K}]^{+}, \quad[\mathrm{SM}(34: 1 ; 2)+\mathrm{H}]^{+}, \quad\left[\mathrm{PE}(36: 4)+{ }^{13} \mathrm{C}+\mathrm{H}\right]^{+}, \quad[\mathrm{PC}(32: 0)+\mathrm{Na}]^{+}\right.$, $[\mathrm{PC}(34: 1)+\mathrm{H}]^{+}, \quad[\mathrm{PC}(38: 4)+\mathrm{Na}]^{+}, \quad$ and $\left.\quad[\mathrm{PC}(38: 4)+\mathrm{K}]^{+}\right) . \quad$ After this internal calibration, all scans were summed (in the mass domain), which generated an initial peak list of 2,643 above the $6 \sigma$ noise limit. This list was then submitted to ALEX123 for identification. We tentatively identify 702 monoistopic lipid peaks in positive-ion mode, which all have mass accuracy values of $\pm 150 \mathrm{ppb}$ (Figure 3.10b). These 702 lipid peaks correspond to 388 unique lipid IDs, after accounting for three possible cations types, which accounts for $26.9 \%$ of the initial peak list. SI Table 1 (not reproduced in this work, please see original publication) contains a full list of these lipids. An additional 1,400 spectral peaks are as isotopologues (typically ${ }^{13} \mathrm{C}$ and ${ }^{13} \mathrm{C}_{2}$ ) of the 702 lipids, which accounts for $\sim 80 \%$ of all peaks. Negative ion mode yielded similar results, where 662 potential monoisotopic lipid peaks (34\%) were identified out of an initial peak list of 1,927. Due to the lower $\mathrm{S} / \mathrm{N}$ of the negative mode spectra, only 738 further peaks were identified as isotopes, for a total of $\sim 72.6 \%$ of all peaks identified.

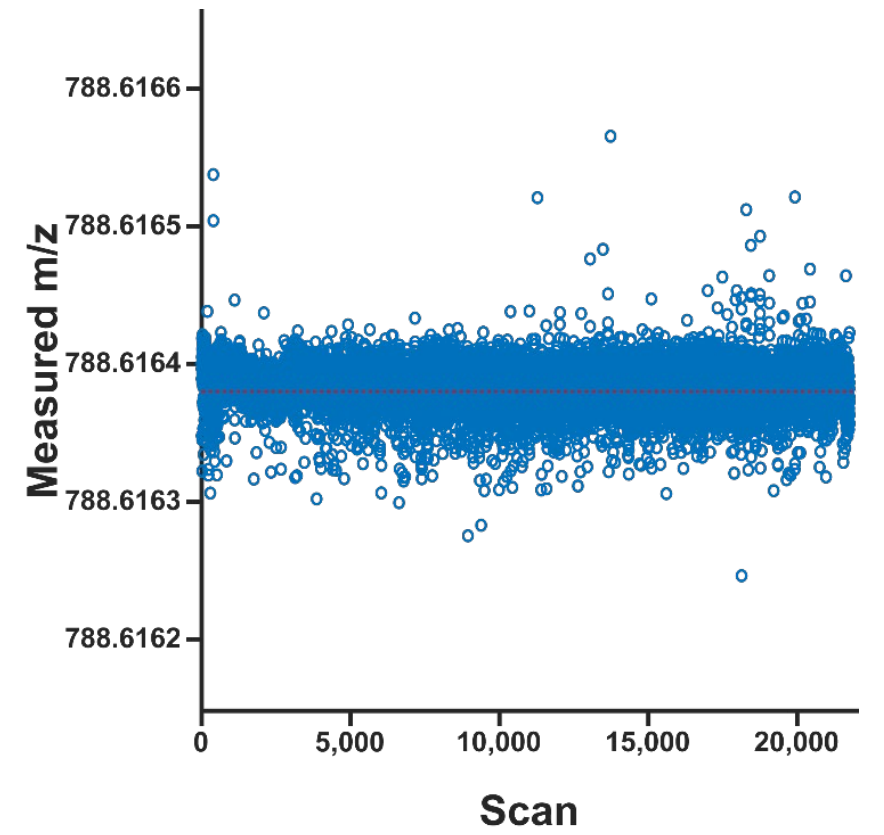

Figure 3.11. Mass accuracy measurement of $\left[\mathrm{PC}(36: 1)+\mathrm{H}^{+}\right]^{+}$. Each dot represents assigned peak in a single spectrum. 
These lipid IDs are supported both by the high mass accuracy $(<150 \mathrm{ppb}$, most $<100 \mathrm{ppb}$ ) and in the positive mode by the intensity of multiple cations for the same species, relative to one another (Figure 3.12a). As protonation, sodiation, and potassiation are all potentially available in brain MSI, we examined the potential to confirm our lipid identifications by comparing all 3 cations. For the most abundant lipid $(\mathrm{PC}(34: 1))$, the $[\mathrm{M}+\mathrm{K}]$ ion has an average ppb error of $16.7,[\mathrm{M}+\mathrm{Na}]-43.5$, and $[\mathrm{M}+\mathrm{H}] 2.2$. While these mass errors are low enough individually to be highly confident in their assignment, having all 3 ions within $60 \mathrm{ppb}$ of one another provides another layer of certainty. Additionally, we can examine the normalized peak intensities of all 3 ions to one another, in this case showing $100 \%, 47.9 \%$, and $21.4 \%$, simplified to a ratio of $4.7: 2.2: 1$. While this insight is not necessarily informative on its own, we can compare this ratio to other PCs, with all the PCs above $3 \%$ of the base peak showing the same ratio (Figure 3.12a). Further, PCs that vary in relative intensity down to $0.2 \%$ of the base peak have generally similar ratios to $\mathrm{PC}(34: 1)$, although as the intensities begin to approach the $6 \sigma$ limit, the ratios begin to deviate and be less similar (Figure 3.12b). One likely scenario for this discrepancy at low $\mathrm{S} / \mathrm{N}$ is that as peaks for any given scan drop below the $6 \sigma$ threshold, the least abundant ions are ignored, leading to sum signal magnitudes in the averaged spectrum that are slightly erroneous. However, as the relative ratios of the three cations are invariable across three orders of magnitude, it improves our certainty that each identification is correct for all lipids within that class. While we observe no alterations to this ratio in the abundant lipid classes, theoretically alterations to this standard ratio could indicate greater abundance of a given lipid within different brain structures (i.e., within the ventricle space rather than within gray or white matter). It is worthwhile to further explore the potentials here, and whether there are observable changes to this ratio between other brain tissues. 


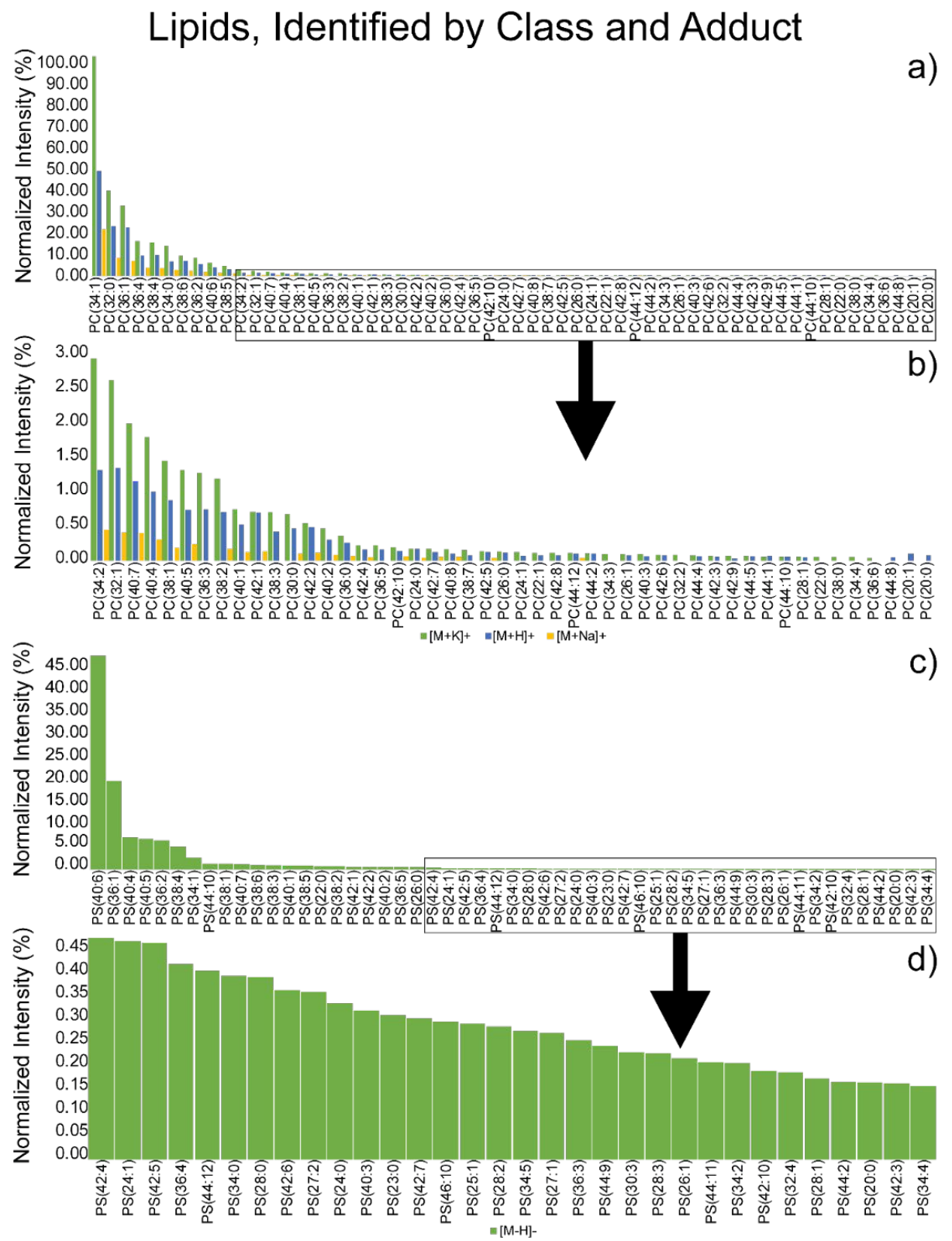

Figure 3.12. Relative abundance of identified lipids by cation and anion for selected classes. In the positive mode, the three major cations (proton, sodium, and potassium) are aligned next to one another, showing the same relative percentages between species, from the most abundant species (PC 34:1) and the other PCs above 3\% (a), as well as for the lower abundant species down to the least abundant species with all three cations represented, PC 44:12 (b). The relative ionization rate between $\mathrm{K}^{+}, \mathrm{H}^{+}$, and $\mathrm{Na}^{+}$hold strictly true down to $1.5 \%$, and generally true down to $0.05 \%$. While the dynamic range is lower for negative mode, we see many potential identifications for many lipid classes (c). We further observe a similar ability to identify potential lipids as low as $0.15 \%$ of the most abundant peak (PI 38:4), for a range of nearly 3 orders of magnitude from the summed spectra (d). 
Additionally, we observe that other lipid classes show similar, though slightly different ratios (Figure 3.13), potentially related to the changes in brain tissue. Negative ionization does not typically have multiple ions of the same species (with deprotonation being the only common method of generating lipid anions unless dopants are added[277, 278]); however, between the most abundant phosphatidylserine (PS(40:6)) and the least abundant (PS(34:4)) there is only a change in ppb error of 17.3 despite a change in intensity of more than an order of magnitude (Figure 3.12c). 

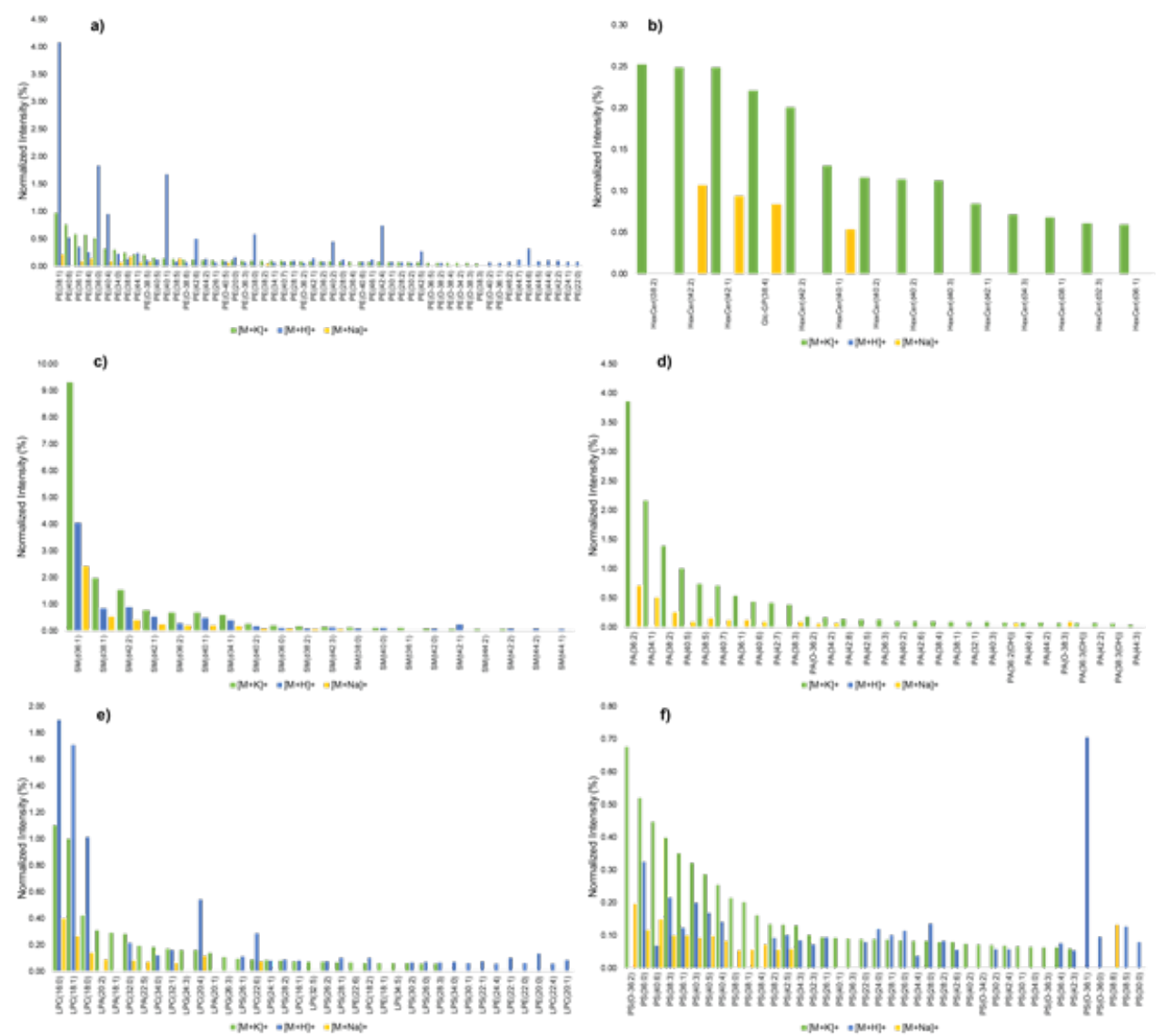

Figure 3.13. Positive mode lipid class identifications, with potassium, sodium, and proton cationization. The lipids follow the same general trends as found in the more abundant PC lipid class, although as the relative intensity of the lipids decrease the ratio between different cations breaks down, as highlighted by several PEs with $\mathrm{H}^{+}$ cationized species several times more intense than their potassiated counterparts (a). Several classes also demonstrate no protonation, due to the potential lack of sites for such ionization (b and d). Sphingomyelin follows the closest trend to the PC class, with a K:H:Na decrease for each lipid identified (c). The lysophospholipids and PS show the most marked diversion for the number of lipids potentially identified in those classes (e and f).

\section{CONCLUSION}

We have demonstrated the utility of combining MSI workflows with a 21 T FT-ICR mass spectrometer. The high magnetic field, combined with a state-ofthe-art ICR cell design provides ultra-high mass resolving power, ppb mass measurement accuracy, and high sensitivity for molecular imaging studies. This advanced instrumentation will pave the way for better understanding of the molecular structure of many tissue types, as well as clarifying current ambiguities 
in MSI. The unique capabilities of this instrument have not yet been fully utilized: on-line tandem mass spectrometry is possible via collision induced dissociation in the linear ion trap, or in the ICR cell via infrared multiphoton dissociation or ultraviolet photo dissociation. Further, the use of harmonic detection cells would further increase the speed of acquisition in these experiments or allow for even higher mass resolving power in a similar time frame. Combined with data-driven MSI acquisition techniques (such as Data-Dependent Acquisition), this instrument promises the most information per unit time of any MSI platform. The estimated number of charges sent to the ICR cell in these experiments is $\sim 4 \times 10^{5}$, based on the mass spectral calibration parameters. The $21 \mathrm{~T}$ FT-ICR routinely operates with ion targets of $1-3 \times 10^{6}$, so additional improvement in dynamic range per pixel is expected. Further, we aim to leverage the unique capabilities of this instrument for other biomolecule imaging experiments, such as metabolites, tryptic peptides, and intact proteins. The 21T FT-ICR is available free of charge to all qualified users as part of the NSF High Field FT-ICR Mass Spectrometry User Facility. This instrument will provide valuable insight into the molecular complexity of tissues at an unprecedented mass spectral resolution, allowing for greater insight into the true distribution of all molecules, as well as accelerating the identification of subtle changes hidden within tissues. 


\section{Chapter 4 EVALUATION OF LIPID COVERAGE AND High SPATIAL RESOLUTION MALDI-IMAGING CAPABILITIES OF OVERSAMPLING COMBINED WITH LASER POST- IONIZATION}

Andrew. P Bowman ${ }^{1}$, Jeroen F. J Bogie ${ }^{2}$, Jerome J.A. Hendriks ${ }^{2}$, Mansour Haidar ${ }^{2}$ Mikhail Belov ${ }^{3}$, Ron M. A. Heeren ${ }^{1}$ and Shane R. Ellis ${ }^{1 *}$

${ }^{1}$ Maastricht MultiModal Molecular Imaging (M4I) Institute, Division of Imaging Mass Spectrometry (IMS), Maastricht University, Universiteitssingel 50, Maastricht 6629ER, The Netherlands

${ }^{2}$ Department of Immunology and Biochemistry, Biomedical Research Institute, Hasselt University, Diepenbeek, Belgium.

${ }^{3}$ Spectroglyph LLC, Kennewick, Washington 99338, United States 


\begin{abstract}
Matrix-Assisted Laser Desorption/Ionization-Mass Spectrometry Imaging (MALDI-MSI) is a powerful technique for visualizing the spatial locations of lipids in biological tissues. However, a major challenge in interpreting the biological significance of local lipid compositions and distributions detected using MALDIMSI is the difficulty in associating spectra with cellular- lipid metabolism within the tissue. By-and-large this is due to the typically limited spatial resolution of MALDI-MSI $(30-100 \mu \mathrm{m})$ meaning individual spectra represent the average spectrum acquired from multiple adjacent cells, each potentially possessing a unique lipid composition and biological function. Use of oversampling is one promising approach to decrease the sampling area and improve the spatial resolution in MALDI-MSI, but it can suffer from a dramatically decreased sensitivity. In this work we overcome these challenges through the first coupling of oversampling MALDI-MSI with laser-post-ionization (MALDI-2). We demonstrate the ability to acquire rich lipid spectra from pixels as small as $6 \mu \mathrm{m}$, equivalent to or smaller than the size of typical mammalian cells. Coupled with an approach for automated lipid identification, it is shown that MALDI-2 combined with oversampling at $6 \mu \mathrm{m}$ pixel size can detect up to three times more lipids and many more lipid classes than even conventional MALDI at $20 \mu \mathrm{m}$ resolution in the positive-ion mode. Applying this to mouse kidney and human brain tissue containing active multiple sclerosis lesions, where 74 and 147 unique lipids are identified, respectively, the localization of lipid signals to individual tubuli within the kidney and lipid droplets with lesion-specific macrophages is demonstrated.
\end{abstract}




\section{INTRODUCTION}

Matrix-Assisted Laser Desorption/Ionization-Mass Spectrometry Imaging (MALDI-MSI) is a powerful method for visualizing the spatial distributions of lipids throughout biological tissues [279-281]. Its versatility for mapping changing lipid compositions within tissues has been demonstrated in a variety of applications, including oncology [282-284], bacterial infections [285, 286] and liver disease [287, 288], amongst many others. The heterogeneous lipid compositions observed with MSI are ultimately the result of cellular-level lipid metabolism occurring within the cells constituting the tissue sample. However, to-date, detailed interpretation of lipid MSI data in terms of cellular-level lipid metabolism has been a major challenge, due to limitations in both technology and informatics. One of the most significant limitations has been the relatively low spatial resolution of conventional methods. Typical experiments are performed at pixel sizes of $\sim 30-100 \mu \mathrm{m}$, significantly larger than most mammalian cells. As a result, each pixel (spectrum) represents the averaged lipid profile acquired from multiple adjacent cells, each potentially possessing distinct metabolic hallmarks and biological functions. This precludes measuring a lipid profile that is reflective of any individual cell, or even single cell-type, within the tissue.

The main limitation of pixel size in MALDI-MSI is the area of desorption/ionization on the tissue surface, that, in turn, is dependent on the laser spot size. Several groups have reported optical modifications to commercial MALDI-MSI ion sources that have reduced the laser spot size, and thus achievable pixel sizes, down to $<10 \mu \mathrm{m}$ and at best $\sim 1 \mu \mathrm{m}$ [289-293]. For example, Kompauer et al recently reported a modified atmospheric pressure MALDI-MSI source capable of reaching $1.4 \mu \mathrm{m}$ pixel size and imaging lipids within single cells [290]. A drawback of these approaches for reducing laser spot size is the requirement of modifications to instrument optics, and the relatively short depth of field of narrowly focused lasers. The latter issue can potentially render the approach sensitive to sample topology or imperfect flatness of the sample stage, which can lead to changing laser fluence and ionization efficiency across the sample surface. Although recent developments in autofocusing methods to compensate for sample topography can help ameliorate these effects, these are not yet widely used [294].

An alternative method to improve spatial resolution of MALDI-MSI is through the oversampling approach [295]. In oversampling, once all material at 
given sampling position has been desorbed/ablated the sample stage is moved by a distance smaller than the laser spot size. As a result, only part of the laser beam is used for desorption/ionization and pixel sizes smaller than the spot size can be achieved. The primary advantage of oversampling is it can be employed on most commercial MALDI systems without hardware modifications, so long as the stage is capable of performing sufficiently small steps. Examples of MALDI-MSI in the oversampling mode include imaging of glycosphingolipids in spleen tissue from a Gaucher disease model at a pixel size of $15 \mu \mathrm{m}$ [296], phospholipids and sulfatides in brain and lung tissue down to $10 \mu \mathrm{m}$ pixel size [297], and human colon tissue at a pixel size of $\sim 5 \mu \mathrm{m}$ [298]. High throughput imaging using continuous raster-mode acquisitions can also generate ions under oversampling-like conditions, where only the edge of the laser beam is generating signal [299], although it has been suggested that severe oversampling conditions can lead to reduced sensitivity in raster-mode MSI [300]. In addition to MALDI, oversampling has also been utilized in IRMALDESI experiments where a $10 \mu \mathrm{m}$ pixel size was achieved for cholesterol imaging from human cervical tissue, although the authors noted that at such pixel sizes a dramatic decrease in sensitivity and lipid coverage was observed [301].

A challenge with oversampling using conventional MALDI lasers with Gaussian-like intensity profiles is that only the edges of the laser spot, where the fluence is lowest, is available for desorption/ionization. This can lead to conditions where, although matrix and analyte are desorbed from the surface, analyte molecules are not efficiently ionized. Such effects can be particularly significant in the conventional pixel-by-pixel acquisition mode [302]. An innovative option to overcome this decreased ionization efficiency is through the use of laser postionization combined with MALDI-MSI (so-called MALDI-2). MALDI-2 has been demonstrated to enable an up to two order of magnitude increase in sensitivity for lipids and other molecular classes from biological tissues [144, 214, 303]. Recently MALDI-2 has been combined with transmission mode MSI to enable spatial resolutions as low as $600 \mathrm{~nm}$ under oversampling conditions [80], but it has not yet been evaluated for oversampling using conventional front-side MALDI laser introduction.

In this work we evaluate for the first time the use of oversampling MALDIMSI combined with MALDI-2 for the imaging of lipids on a high resolution Orbitrap mass spectrometer. Exploiting the increased ionization efficiency enabled by MALDI-2 we demonstrate the ability to generate rich lipid signals from pixel 
sizes as low as $6 \mu \mathrm{m}$ from an original laser spot size of $\sim 15 \mu \mathrm{m}$. In addition, using an automated lipid identification workflow we have studied the types and numbers of lipid species that can be detected using MALDI and MALDI-2 in both conventional and oversampling imaging modes. This provides what is to-date the most comprehensive overview of lipid detection using MALDI-2. The utility of this method for high content and high spatial resolution lipid imaging using $6 \mu \mathrm{m}$ pixel sizes is demonstrated using rat liver, mouse kidney and human brain tissue containing active multiple sclerosis lesions, where localization of lipid signal to individual cellular-level features is found. This unique combination of high mass accuracy, high mass resolving power, high spatial resolution, and enhanced sensitivity provides an exciting method to study lipid metabolism at the cellular level within heterogeneous and complex tissue sections.

\section{Methods}

\section{MATERIALS}

Isopropanol (LC-MS grade), ethanol (LC-MS grade), 2,5Dihydroxybenzoic acid (DHB, $\geq 99.9 \%$ purity), 2,5-Dihydroxyacetophenone (DHA) and water (LC-MS grade) were purchased from Sigma Aldrich (Zwijndrecht, The Netherlands), and used without further purification. Hematoxylin (Merck, Darmstadt, Germany) and eosin Y (J.T. Baker, Center Valley, PA, USA) were used under standard laboratory protocols. Indium tin oxide (ITO)coated glass slides were purchased from Delta Technologies (Loveland, USA).

\section{BIOLOGICAL SAMPLES}

Healthy rat liver was obtained from Maastricht University in accordance with protocols approved by the Animal Care and Use Committee (DEC number 2014-120) and was from the same animal used in a recent study [304]. Rats were provided ad libitum access to water and regular chow. One mouse kidney was collected from an LDL R-/- mice from a protocol approved by the Animal Care and Use Committee (DEC number 2014-069) at Maastricht University. Mice were provided ad libitum access water and regular chow. Animals were housed and cared for at the Central Animal Facility of Maastricht University according to local standards. $12-\mu \mathrm{m}$ thick sections were prepared using a cryo-microtome (Leica, Nussloch, Germany) at $-20{ }^{\circ} \mathrm{C}$ and thaw-mounted on ITO-coated glass slides. Sections were stored at $-80^{\circ} \mathrm{C}$ until analysis which occurred approximately 6 
months after sectioning. Human brain tissue with active multiple sclerosis (MS) lesions were obtained from the Netherlands Brain Bank (NBB, Amsterdam, Netherlands). The Netherlands Brain Bank received permission to perform autopsies for the use of tissue and for access to medical records for research purposes from the Ethical Committee of the VU University Medical Center, Amsterdam, The Netherlands. The experiments protocols and methods used for analyzing active MS lesions were conducted with the approval of the Netherlands Brain Bank and the Medical Ethical Committee Hasselt University, and carried out according to institutional guidelines. Brain tissue was stored at $-80^{\circ} \mathrm{C}$ until sectioning. $10-\mu \mathrm{m}$ thick sections were prepared using a cryo-microtome and were thaw-mounted onto standard glass slides, transported to Maastricht University on dry ice and then again stored at $-80^{\circ} \mathrm{C}$ until matrix application and MSI analysis. The time between sectioning and analysis was less than one month.

\section{SAMPLE PREPARATION}

Matrix application was performed via sublimation [305]. Sublimation was performed under the following conditions: $40 \mathrm{mg}$ of DHB dissolved in isopropanol, sublimed for 4 minutes at $160^{\circ} \mathrm{C}$ at a pressure of $<4 \times 10^{-5}$ bar. Samples that were coated in DHA followed slightly different conditions: $40 \mathrm{mg}$ of DHA dissolved in acetone, sublimed for 4 minutes at $140{ }^{\circ} \mathrm{C}$ at a pressure of $<4 \times 10^{-5}$ bar. Samples were then recrystallized in a lab-made apparatus containing $1 \mathrm{~mL}$ of $0.5 \%$ ethanol in water, at $50{ }^{\circ} \mathrm{C}$ for 90 seconds.

$\mathrm{H} \& \mathrm{E}$ staining was performed on kidney sections after MALDI imaging. Matrix coated tissue sections were cleaned of remaining MALDI matrix by immersion in $100 \%$ ethanol for 20 seconds. A standard $\mathrm{H} \& \mathrm{E}$ protocol was then used $\left(95 \% \mathrm{EtOH}, 70 \% \mathrm{EtOH}, \mathrm{H}_{2} \mathrm{O}\right.$ for 30 s each, hematoxylin for 3 minutes, $\mathrm{H}_{2} \mathrm{O}$, $70 \% \mathrm{EtOH}, 95 \% \mathrm{EtOH}$ each for 30s, eosin for 1 minute, $95 \%$ and $100 \% \mathrm{EtOH}$ for 30 s each, xylene for 2 minutes). High resolution optical images of stained tissues were generated using a Mirax Desk scanner (3DHistech, Budapest, Hungary). Unfixed cryosections of human brain tissue were stained with $0.3 \%$ Oil Red O (ORO, Sigma) for $10 \mathrm{~min}$ to visualize neutral lipids (cholesterol esters). Counterstaining of cell nuclei was done using hematoxylin incubation. Analysis was carried out using a Leica DM 2000 LED microscope and ImageJ software.

CD68 immunostaining and analysis was performed on fixed cryosections of human brain tissue using the antibody anti-CD68 (1:100, cat. \#14-0688, 
Invitrogen) and a species compatible Alexa647 secondary anti-body (Life Technologies, A21247) as described previously [306]. To label the myelinated areas, the immune-stained sections were subsequently incubated for 30 minutes at RT with $2 \mu$ M Bodipy ${ }^{\circledR} 493 / 503$ solution (ThermoFisher Scientific, D3922) diluted in PBS. Analysis was then carried out using a Nikon eclipse 80i microscope.

\section{MASS SPECTROMETRY INSTRUMENTATION}

All MSI experiments were performed on an Orbitrap Elite mass spectrometer (Thermo Fisher Scientific GmbH, Bremen, Germany) coupled to a reduced pressure ESI/MALDI ion source (Spectroglyph LLC, Kennewick, WA, USA). Further details on the ion source can be found in [307]. The $349 \mathrm{~nm}$ MALDI laser (Spectra Physics, Mountain View, CA, USA) was operated at a repetition rate of $100 \mathrm{~Hz}$ and pulse energy of $\sim 1.0 \mu \mathrm{J}$. The laser was focused to a spot size of $\sim 15 \times 12$ um as determined by the size of ablation craters in a matrix layer (Figure 4.1). Laser post-ionization (MALDI-2) was performed as previously described for the same experimental setup [214]. Briefly post-ionization was achieved using a wavelength tunable optical parametric oscillator laser system (Ekspla NT-230, Vilnius, Lithuania). The MALDI-2 laser was operated at $260 \mathrm{~nm}$ with a postattenuation pulse energy of $500 \mu \mathrm{J}$. Using adjustable mirrors, the beam was guided to be parallel with and $\sim 250-400 \mu \mathrm{m}$ above the sample surface where it intersected the desorbed plume generated by the MALDI laser pulse. OPO laser emission was synchronized with the MALDI laser using a digital pulse/delay generator (DG645, Stanford Research Systems, Sunnyvale, USA). Emission from the OPO laser occurred $20 \mu$ s after each MALDI laser pulse. The mass spectrometer was operated in positive-ion mode using an ion injection time of $250 \mathrm{~ms}$, automatic gain control (AGC) turned off and a mass range of 350-2000. 


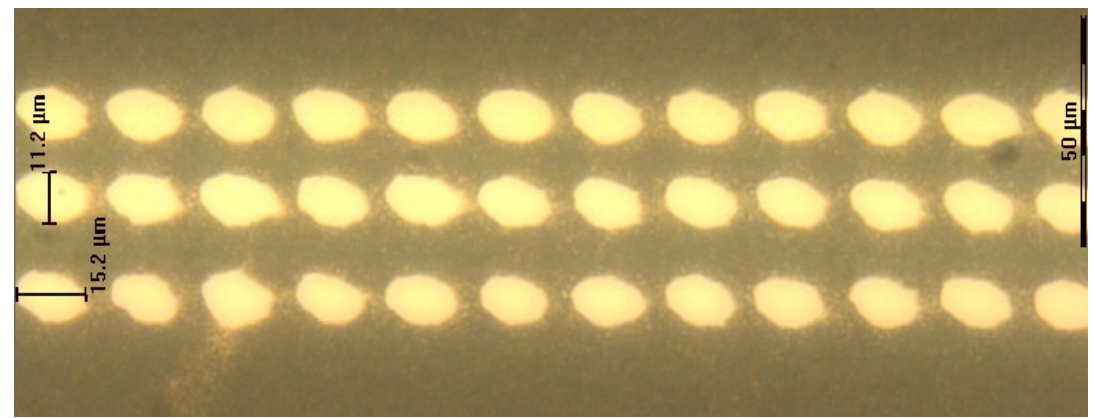

Figure 4.1 Optical image of ablation craters generated by the MALDI laser on a DHB-coated slide. MALDI laser spot size is $\sim 11 \mu \mathrm{m} \times 15 \mu \mathrm{m}$. Laser was operated at $1.1 \mu \mathrm{J}$ and a repetition rate of $100 \mathrm{~Hz}$. Stage step size was 20 $\mu \mathrm{m}$.

\section{DATA ACQUISITION}

Line scans of rat liver: Rat liver tissue was used for comparison of MALDI and MALDI-2 data generated at different step sizes. Three sets of alternate MALDI and MALDI-2 line scans were acquired in a single experiment (i.e., a line of MALDI data followed by a line of MALDI-2 data) and performed at 20, 15, 10, 8 , 6 , and $4 \mu \mathrm{m}$ step sizes for both DHB and DHA-coated tissues. As the first row in each acquisition does not result in oversampling in the vertical direction, one extra row was acquired at the start of each acquisition but not used for data analysis. This ensured each line scan was acquired under representative oversampling conditions. Line scans were performed using a mass resolution setting of setting of 240,000 (FWHM@m/z 400) giving a total scan time of $1.06 \mathrm{~s} / \mathrm{scan}$.

Imaging of mouse kidney and human brain tissue: Human brain tissue containing active multiple sclerosis lesions and mouse kidney tissues were coated in DHB matrix and analyzed using MALDI-2-MSI at a step size of $6 \mu \mathrm{m}$ and a mass resolution of 120,000 (FWHM @ m/z 400), giving a total scan time of 0.67 $\mathrm{s} / \mathrm{scan}$. As with the line scans, the first row of each dataset was removed so that all rows were acquired under the same oversampling conditions. To support the identifications of several unexpected compounds ion trap MS/MS spectra were acquired from one human brain and one kidney tissue section using the DDAimaging method [308].

\section{DATA ANALYSIS AND LIPID IDENTIFICATION}

All data image visualization and data analysis were performed using LipostarMSI (Molecular Horizon Srl, Bettona, Italy). Prior to import all proprietary 
Thermo Fisher .raw data was converted into imzML [309]. This was done by first converting raw data into mzML using msconvert (ProteoWizard) [268]. Using the in-built converter of LipostarMSI the mzML file was then combined with the positioning file created by the MALDI/ESI Injector to generate a profile mode imzML file. Lipid identification within LipostarMSI was performed with reference to the LIPIDMAPS database (.sdf format) [310] and was based on accurate $\mathrm{m} / \mathrm{z}$ matching using a tolerance of $\pm 2 \mathrm{ppm}$. All lipid identifications are therefore reported to the sum-composition level. Phospholipids, sphingolipids, and sterols were considered for identification. Note that in the case of sterols many isomeric species are possible, and we thus group all $\mathrm{m} / \mathrm{z}$ matches to a general "sterol" group, while for ether phospholipids, identifications containing isomeric acyl and alkenyl linkages were grouped into a general ether sub-group (e.g., PE-O and PC-O). In the case of MALDI analysis of liver tissue, potassiated species dominated the spectra, while MALDI-2 spectra were dominated by protonated species, consistent with prior observations comparing MALDI and MALDI-2 [144, 214]. Therefore, to avoid the occurrence of the same lipid being detected as multiple adducts and counting as multiple identifications, only $[\mathrm{M}+\mathrm{K}]^{+}$ions of phospholipids and sphingolipids were considered for MALDI data, and only $[\mathrm{M}+\mathrm{H}]^{+}$ions considered for MALDI-2 data. Sterols were searched for as $\left[\mathrm{M}+\mathrm{H}-\mathrm{H}_{2} \mathrm{O}\right]^{+}$ions for both MALDI and MALDI-2, apart from cholesterol esters (CE) for the analysis of human brain tissue (see below). Several sub-classes unlikely to be observed in positive-ion mode data (e.g., sulfatides and cardiolipins) were removed from the search list and only identifications corresponding to even-numbers acyl/ether chains were considered to limit false positives. Further sample-specific parameters are provided below.

Line scans of rat liver. Each line scan was converted to imzML file consisting of 75 pixels (spectra) using a dummy .xml position file. This resulted in 3 MALDI and 3 MALDI-2 datasets per step size and matrix type. While no lower limit was set for peak intensity for peak picking during data import, the minimum peak frequency was set to $50 \%$ (meaning that peaks had to appear in at least half of the pixels in any given line) with a tolerance for peak alignment of $3 \mathrm{ppm}$. In this way only reproducible signals were considered, and very low abundance peaks close to the detection limit and/or corresponding to random electronic noise were discarded. All scans were recalibrated using up to 3 peaks, [Cholesterol- $\left.\mathrm{H}_{2} \mathrm{O}+\mathrm{H}\right]^{+}$, $[\mathrm{PE}(38: 4)+\mathrm{H}]^{+}$, and $[\mathrm{PC}(34: 2)+\mathrm{K}]^{+}$, during data import. The final ID list was then manually curated, and several seemingly spurious identifications removed. Single- 
scan noise values were taken from Xcalibur Qual Browser 2.3 (Thermo Fisher Scientific GmbH, Bremen, Germany).

Imaging of mouse kidney and human brain tissue: Import of imzML files to LipostarMSI for the kidney and brain samples was performed using the following parameters: intensity threshold of $1 \%$ of base peak; peak alignment tolerance of 3 ppm; peak detection frequency of $2 \%$; and a minimum spatial chaos value of 0.7 (with a value of 1 corresponding to high image structure and a value of 0 to a random (structureless) spatial distribution). Identification followed an identical process outlined above, apart from $[\mathrm{M}+\mathrm{K}]^{+}$ions of CEs also being considered in the brain data. These were added following manual interrogation of the raw data and the observation of several localized and abundant potassiated CE signals. In addition to manual curation of identified lipid species as outlined for liver line scans above, we also only kept identifications that displayed tissue-specific distributions. Several antioxidant species observed as radical cations from kidney tissue were manually annotated but not counted in the final identification lists as these identifications were not automated (LipostarMSI does not yet support radical cations as a search class).

MSI data was visualized following total ion current normalization and applying hotspot removal (high quantile 99\%).

SCANNING ELECTRON Microscopy

Scanning electron microscopy images of matrix-coated kidney tissue after DHB sublimation and recrystallization were acquired using a Philips XL30 microscope.

RESULTS AND DISCUSSION

LIPID COVERAGE WITH OVERSAMPLING COUPLED WITH MALDI-2 FROM LIVER TISSUE 
a) 800000

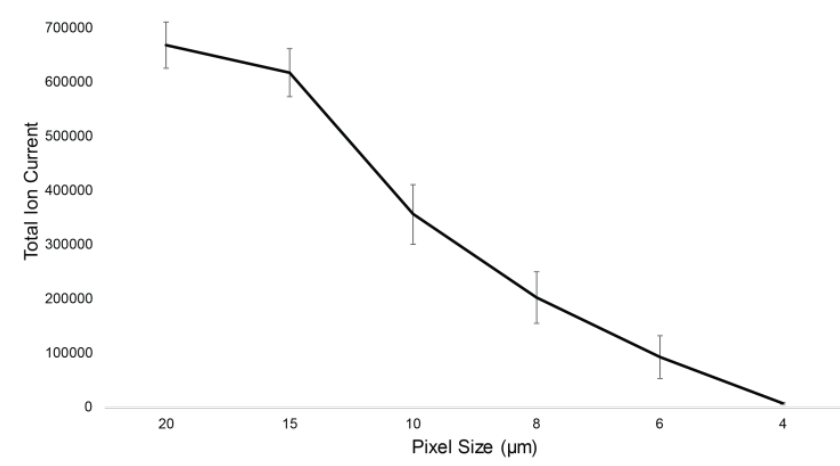

b) 80000

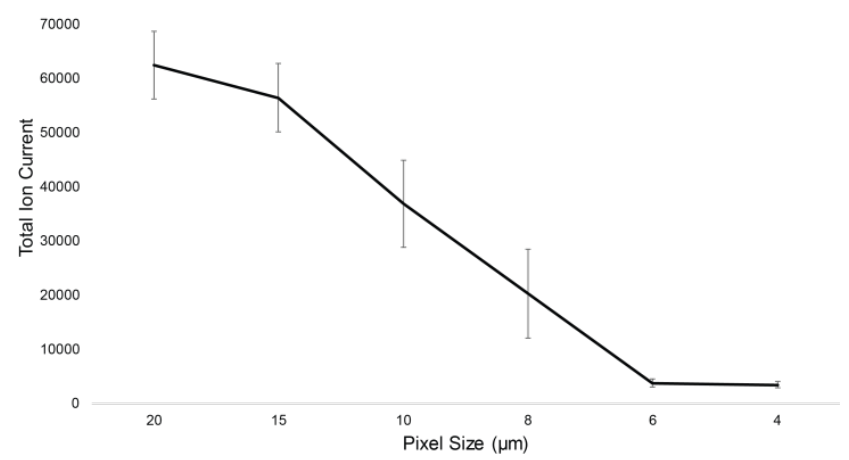

Figure 4.2. Total ion current recorded between m/z 350-2000 for (a) MALDI-2 and (b) MALDI analysis rat liver tissue at different stage step (pixel) sizes. Error bars represent the \pm 1 standard deviation of all scans $(>150$ individual scans) acquired at a given step size.

Data for both MALDI and MALDI-2 spectra were generated using stage step sizes of $20 \mu \mathrm{m}$ (no oversampling) and $6 \mu \mathrm{m}$ (significant oversampling) from line scans of liver tissue to compare the number and types of lipid species detected. The spot size of the MALDI laser on the tissue was $\sim 15 \mu \mathrm{m} \times 11 \mu \mathrm{m}$ (Figure 4.1). As expected, at smaller step sizes the total-ion current and overall signal-to-noise $(\mathrm{S} / \mathrm{N})$ decreases for both MALDI and MALDI-2 data (Figure 4.2). When using a 4 $\mu \mathrm{m}$ step size lipid signals were low and unstable, thus $4 \mu \mathrm{m}$ data was not explored further. 
a) $20 \mu \mathrm{m}$ step size (no oversampling)

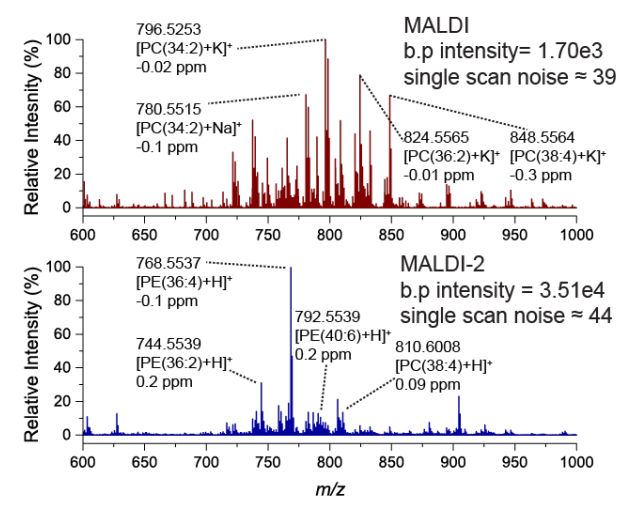

c) Detected lipid species

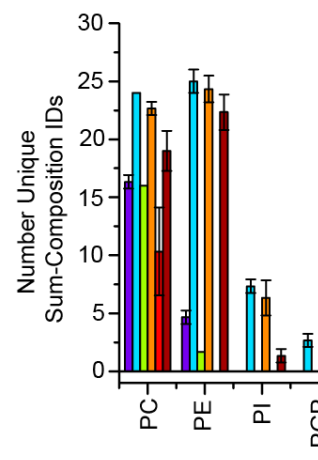

Figure 4.3 MALDI (top, red trace) and MALDI-2 (bottom, blue trace) spectra acquired from rat liver tissue coated in DHB matrix using stage step sizes of (a) $20 \mu \mathrm{m}$ (no oversampling) and (b) $6 \mu \mathrm{m}$ (with oversampling). Each spectrum is the average of 10 consecutive scans. (c) The number of detected lipid species across different lipid classes observed using MALDI and MALDI-2 using 20, 10 and $6 \mu \mathrm{m}$ line scans across liver tissue. Peaks used for identification had a detection frequency $\geq 50 \%$ across the line scans consisting of 75 pixels (equivalent to being detected in half or more individual scans). $[\mathrm{M}+\mathrm{K}]^{+}$ions were considered for MALDI identification and $[\mathrm{M}+\mathrm{H}]^{+}$ ions considered for MALDI-2 identifications, with the exception for sterols which were identified in both cases as $\left[\mathrm{M}+\mathrm{H}-\mathrm{H}_{2} \mathrm{O}\right]^{+}$ions. Error bars represent \pm 1 standard deviation across three replicate line scans. b.p. $=$ base peak.

Figure 4.3a shows representative MALDI spectra from rat liver tissue coated with DHB matrix using a step size of $20 \mu \mathrm{m}$ (10 consecutive scans averaged with single scan $\mathrm{S} / \mathrm{N}$ values indicated, $m / \mathrm{z}$ 600-1000 shown). Full mass range spectra can be found in the Figure 4.4 and Figure 4.5. As typically observed, MALDI spectra are dominated by phosphatidylcholine (PC) and sphingomyelin (SM) lipids, with their potassiated adducts being most abundant. By contrast, MALDI-2 spectra generated under analogous conditions (Figure 4.3a, bottom) resulted in both a $c a, 200$-fold increase in base peak intensity and dramatic 
increases in signal for a variety of lipid species, while lipid-related signals also became dominated by $[\mathrm{M}+\mathrm{H}]^{+}$ions, consistent with prior observations [144, 214].
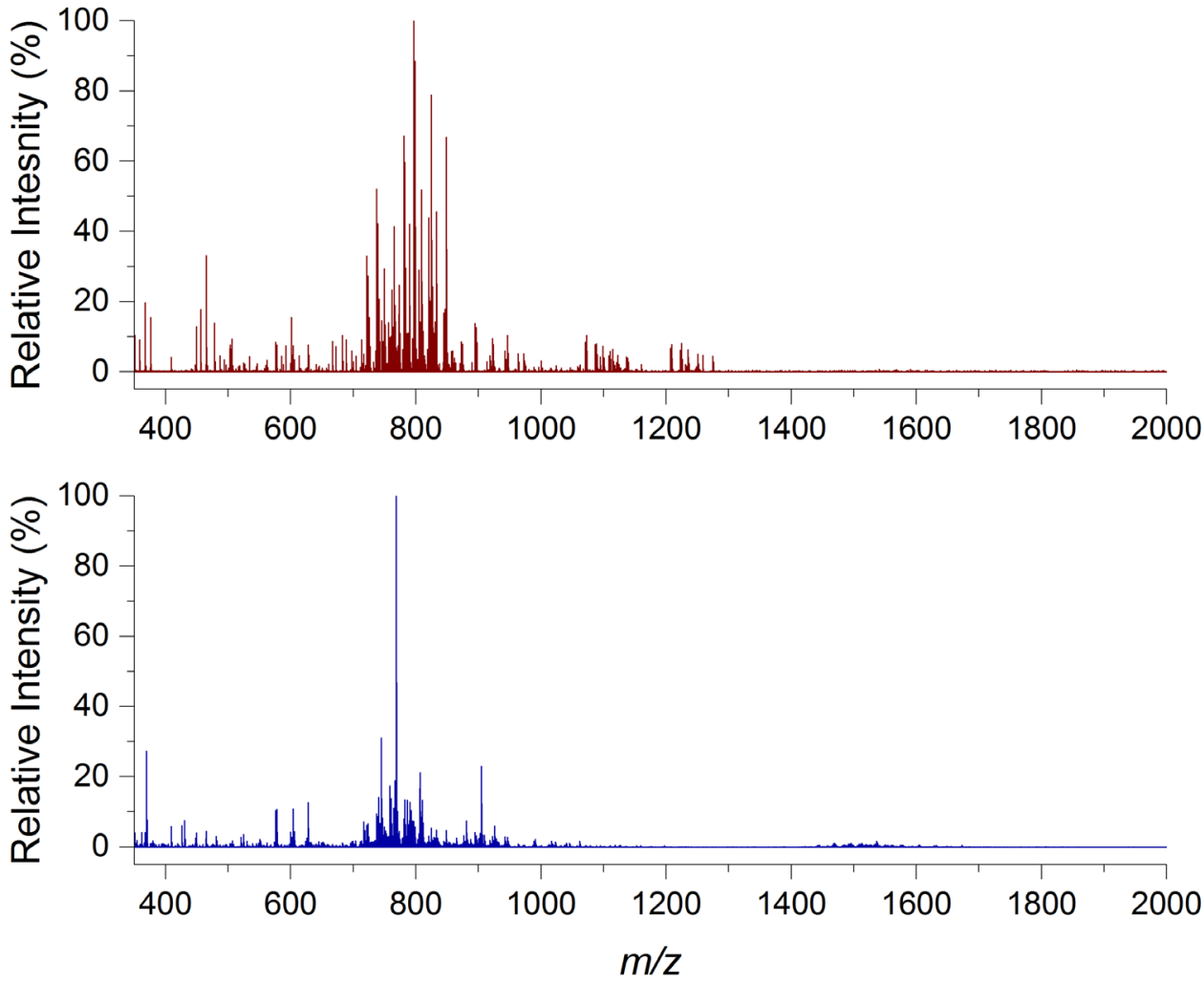

Figure 4.4. MALDI (top, red trace) and MALDI-2 (bottom, blue trace) spectra acquired from rat liver tissue using DHB matrix and a $20 \mu \mathrm{m}$ stage step across the full $\mathrm{m} / \mathrm{z} 350-2000$ spectral range. Spectra represent the average of 10 consecutive scans.

The benefit of MALDI-2 is observed to hold even under oversampling conditions. Figure $4.3 \mathrm{~b}$ (top) shows representative MALDI spectra acquired using a $6 \mu \mathrm{m}$ step size that results in significant oversampling. While the overall spectrum resembles that shown in the top panel of Figure 4.3a, the signal intensity is reduced by 10 -fold with many peaks no longer being detected. However, data acquired using a $6 \mu \mathrm{m}$ step size and MALDI-2 still produces high S/N spectra with base peak intensity $\sim 3$-fold higher than those generated by conventional MALDI at $20 \mu \mathrm{m}$ (Figure $4.3 \mathrm{~b}$, bottom). Using a $6 \mu \mathrm{m}$ step size, the $\mathrm{S} / \mathrm{N}$ of the base peak corresponding to the phosphatidylethanolamine (PE), $[\mathrm{PE}(36: 4)+\mathrm{H}]^{+}$, is 136 using MALDI-2, whereas using the same step size the corresponding lipid detected with 
conventional MALDI, observed as the $[\mathrm{M}+\mathrm{K}]^{+}$ion, has a single scan $\mathrm{S} / \mathrm{N}$ of $<1$. For the abundant $\mathrm{PC}(34: 2)$ lipid the signal intensity for the protonated species acquired at $6 \mu \mathrm{m}$ step size was comparable to that measured for the abundant potassiated species with MALDI at $20 \mu \mathrm{m}$ step size. It is noteworthy for these data acquired at $6 \mu \mathrm{m}$ step size individual spectra are taken from a tissue area equivalent to or smaller than the size of a typical mammalian cell $(10-100 \mu \mathrm{m})$, thereby providing data that is reflective of cellular-level lipid compositions within the tissue. Although we acknowledge that for smaller cells there is a reasonable probability of collecting ion signal from two adjacent cells in a single pixel.
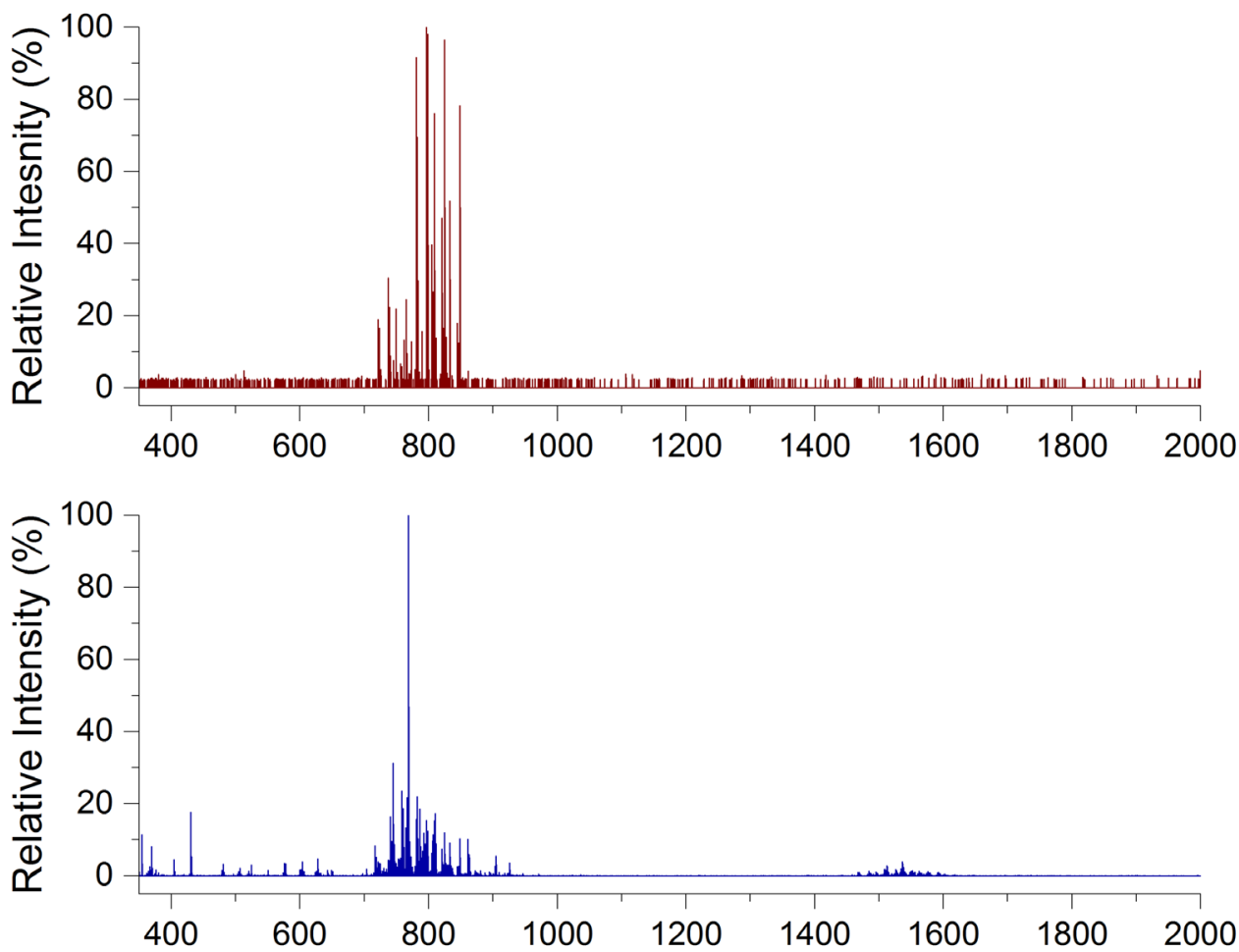

Figure 4.5. MALDI (top, red trace) and MALDI-2 (bottom, blue trace) spectra acquired from rat liver tissue using DHB matrix and a $6 \mu \mathrm{m}$ stage step across the full $\mathrm{m} / \mathrm{z} 350-2000$ spectral range. Spectra represent the average of 10 consecutive scans.

MALDI-2 oversampling was also evaluated using 2,5 DHA matrix (Figure 4.6). Using a $20 \mu \mathrm{m}$ step size MALDI and MALDI-2 spectra were similar to those obtained using DHB. Interestingly though, with an increasing extent of oversampling many of the MALDI-2-specific signals observed with DHB yielded lower relative intensities using DHA. For example, at $6 \mu \mathrm{m}$ step sizes using 
MALDI-2 protonated PC signals yielded the highest signal intensities (rather than PE lipids) while a relative increase in the abundance of potassiated PC lipids compared to their protonated forms was also observed. In contrast, the overall spectral profile at different step sizes remained relatively consistent using DHB (Figure 4.3a and b). The origin of this effect is unclear but appears to suggest a shift of DHA ionization properties under oversampling conditions when using MALDI2. We speculate this effect is related to: (i) the higher volatility of DHA, resulting in a larger desorption area upon irradiation by the MALDI-laser. Upon oversampling this could result in matrix further from the center of the laser spot being desorbed leading to a plume of lower density in which MALDI-2 can occur and/or (ii) possible differences in matrix morphology and diffusion rates of lipids from the tissue with lipids closer to the surface being more selectively desorbed by the edge of the laser beam. Different diffusion rates of lipids have recently been reported in sublimed matrix coatings using secondary ion mass spectrometry [311]. Thorough investigation of this effect lies outside the scope of this work but provide an exciting avenue to pursue that could provide insight into the MALDI-2 mechanism.
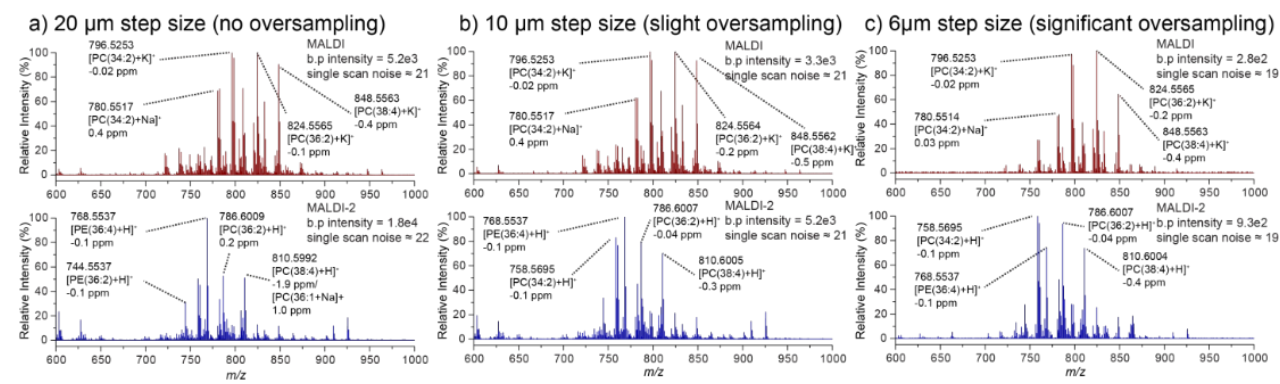

Figure 4.6. MALDI (top, red trace) and MALDI-2 (bottom, blue trace) spectra acquired from rat liver tissue using DHA matrix at stage step sizes of $20 \mu \mathrm{m}$ (left), $10 \mu \mathrm{m}$ (middle) and $6 \mu \mathrm{m}$ (right). Spectra represent the average of 10 consecutive scans. The corresponding base peak (b.p.) intensity and single scan noise values are indicated.

We next evaluated the breadth of lipid coverage that can be obtained in such high spatial resolution MSI experiments using DHB. Peaks used for automated identification were defined during data import as having a peak detection frequency $\geq 50 \%$ (equivalent to being detected in half or more scans/pixels within a $3 \mathrm{ppm}$ tolerance) across each 75 pixel line scan. Figure $4.3 \mathrm{c}$ shows the number of lipid species detected within $2 \mathrm{ppm}$ of their theoretical $\mathrm{m} / \mathrm{z}$ for both MALDI and MALDI-2 at step sizes of $20 \mu \mathrm{m}$ (no oversampling), $10 \mu \mathrm{m}$ (moderate oversampling) and $6 \mu \mathrm{m}$ (significant oversampling). The full list of 
tentatively identified species and their abundances is provided in Electronic Supplementary Material Table S1 (not reproduced here). Conventional MALDI detected primarily PC lipids along with several abundant SM and PE lipids as $[\mathrm{M}+\mathrm{K}]^{+}$ions. In total 24,19 and 11 unique sum-composition lipid species were detected with MALDI at step sizes of 20, 10 and $6 \mu \mathrm{m}$, respectively. Dramatically more lipid species were detected using MALDI-2, with 149, 117 and 66 lipid species being detected as $[\mathrm{M}+\mathrm{H}]^{+}$ions $\left(\left[\mathrm{M}+\mathrm{H}-\mathrm{H}_{2} \mathrm{O}\right]^{+}\right.$for sterols $)$at 20,10 and 6 $\mu \mathrm{m}$ step sizes, respectively. While PE and PE-O species were among the biggest beneficiaries of MALDI-2, it also enabled the detection of a diverse array of lipid classes not observed with conventional MALDI. For example, MALDI-2 could detect both phosphatidylinositol (PI) and phosphatidylserine (PS) lipid species as $[\mathrm{M}+\mathrm{H}]^{+}$ions. These lipids are often only observable in negative-ion mode MALDI analysis thereby demonstrating the added lipid coverage offered by MALDI-2. Supporting these observations is the fact that the most intense protonated species observed for each, PI(38:4) and PS(36:1), have been shown to be the two most abundant species of each class using LC-MS/MS [312]. Despite the expected drop in the number of detected lipids with decreasing pixel size, the above data demonstrates that rich lipid MSI data covering many different lipid species can be generated from pixel sizes as low as $6 \mu \mathrm{m}$, with a $\sim 3$-fold increase in the number of detected lipid species compared to conventional MALDI using a $20 \mu \mathrm{m}$ pixel size. 


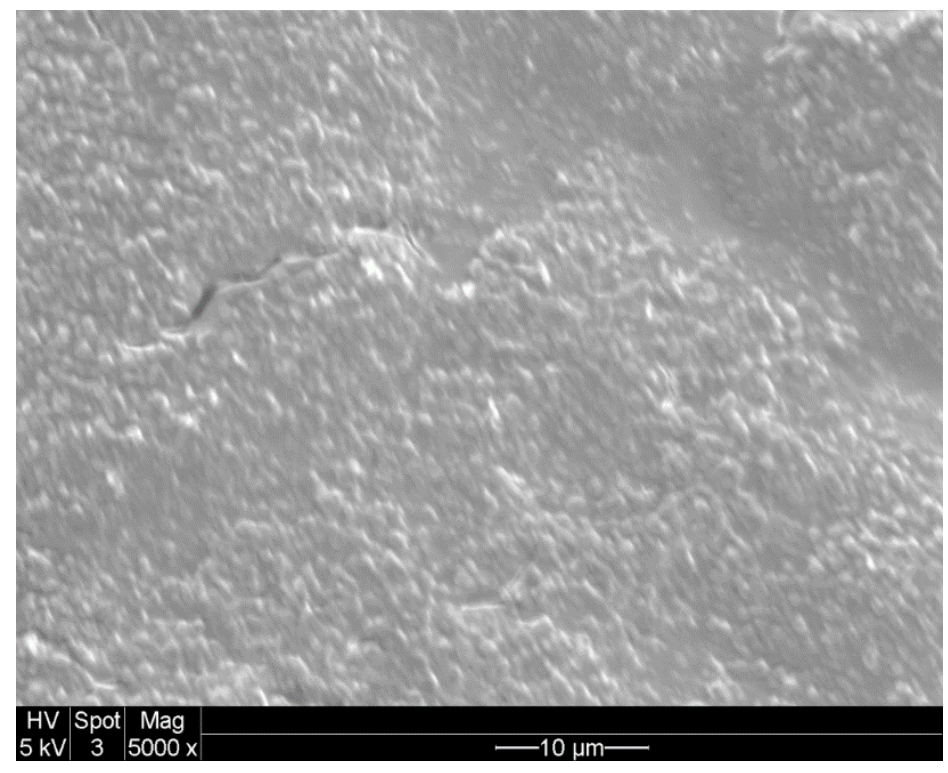

Figure 4.7. Scanning electron microscopy images of mouse kidney tissue coated with DHB matrix. DHB matrix was applied using sublimation and then recrystallized as described in the methods section.

\section{HIGH SPATIAL RESOLUTION IMAGING OF LIPIDS IN KIDNEY TISSUE}

Mouse kidney tissue was used to evaluate the high-resolution imaging capabilities of MALDI-2 oversampling. A $\sim 4 \mathrm{~mm}^{2}$ area of DHB-coated tissue section was analyzed using a $6 \mu \mathrm{m}$ step resulting in an image consisting of 108,558 pixels. Using scanning electron microscopy, the DHB crystal sizes were found to be significantly smaller than $6 \mu \mathrm{m}$ (Figure 4.7). The average spectrum is shown in Figure $4.8 \mathrm{a}$ and demonstrates the detection of a variety of lipid species across the $m / z$ 350-2000 mass range. Using the described approach for automated lipid identification (see Methods) 74 unique sum-composition lipid species were identified as $[\mathrm{M}+\mathrm{H}]^{+}$ions $\left(\left[\mathrm{M}+\mathrm{H}-\mathrm{H}_{2} \mathrm{O}\right]^{+}\right.$ions for sterols $)$from this dataset. The distribution of these identifications across the different lipid sub-classes is shown in Figure 4.8b. As observed for the liver data above PC, PE and PE-O lipids constituted the majority of detected lipid species and in total 12 different lipid subclasses could be identified and imaged. The full list of identified lipids is provided in the original publication. 

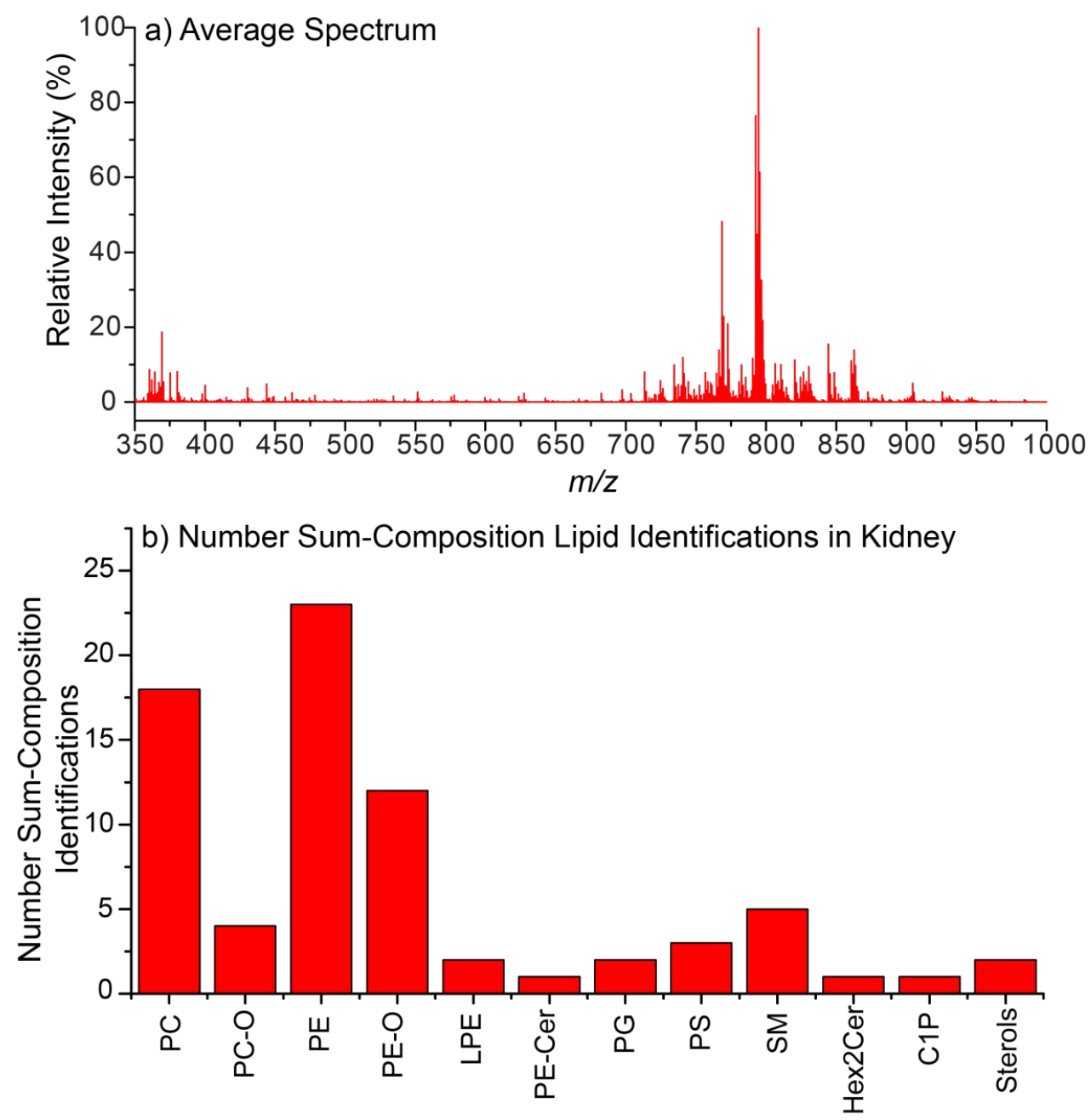

\section{Lipid Class}

Figure 4.8 (a) Average spectrum acquired from mouse kidney tissue using MALDI-2 and a pixel size of $6 \mu \mathrm{m}$ between $\mathrm{m} / \mathrm{z}$ 350-1000. (b) Number of automatically identified lipid species from mouse kidney tissue. Lipids were identified as $[\mathrm{M}+\mathrm{H}]^{+}$ions $\left(\left[\mathrm{M}+\mathrm{H}-\mathrm{H}_{2} \mathrm{O}\right]^{+}\right.$for sterols) using an $m / z$ tolerance of $2 \mathrm{ppm}$.

Figure 4.9 shows an optical image of the post-MSI hematoxylin and eosin (H\&E) stained tissue section (Figure 4.9a) and an overlay of three identified lipid species (Figure 4.9b, $[\mathrm{PC}(38: 6)+\mathrm{H}]^{+}$(green), $[\mathrm{PE}(\mathrm{O}-40: 8)+\mathrm{H}]^{+}$(blue) and $[\mathrm{PE}(\mathrm{O}-$ $36: 5)+\mathrm{H}]^{+}$(pink)). Using the distributions of these three ion signals a number of different tissue regions can be localized at the cellular level. $[\mathrm{PE}(\mathrm{O}-36: 5)+\mathrm{H}]^{+}$is localized to the inner medulla, inner stripe, glomeruli and the interstitium regions of the kidney. Both $[\mathrm{PE}(\mathrm{O}-40: 8)+\mathrm{H}]^{+}$and $[\mathrm{PC}(38: 6)+\mathrm{H}]^{+}$are specific to the kidney 
tubuli, with $\left[\mathrm{PC}(\mathrm{O}-40: 8+\mathrm{H}]^{+}\right.$being more abundant in tubuli contained within the outer stripe of the medulla and $[\mathrm{PC}(38: 6)+\mathrm{H}]^{+}$being more abundant in tubuli contained within the cortex. The high specificity for these lipid signals for histologically different tissue regions can be seen in the zoomed MSI and H\&E data shown in Figure 4.9 c-f. The outlined area of Figure 4.9d (white dotted line) highlights tubuli-specific lipid signals corresponding to the tubular regions outlined in the H\&E-stained tissue with excellent spatial specificity (Figure 4.9f). This strong agreement between the MSI and histological data confirms the high spatial resolution enabled by both the oversampling method and that the employed sample preparation using sublimation minimizes analyte delocalization. Combined, this data demonstrates the ability to achieve pixel sizes on the scale of cellular-level features within tissues while still being able to detect and identify numerous lipid species. 
a)

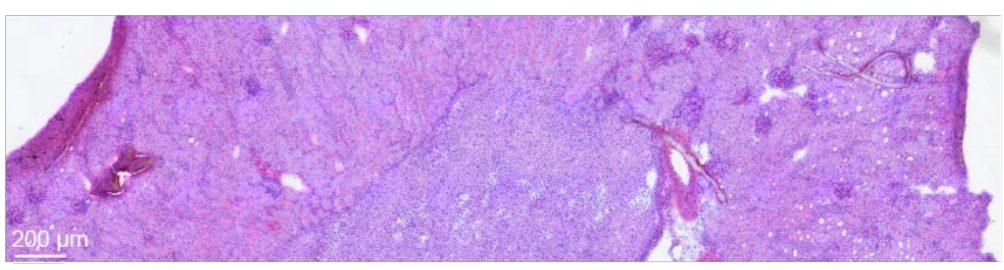

b) $200 \mu \mathrm{m}$

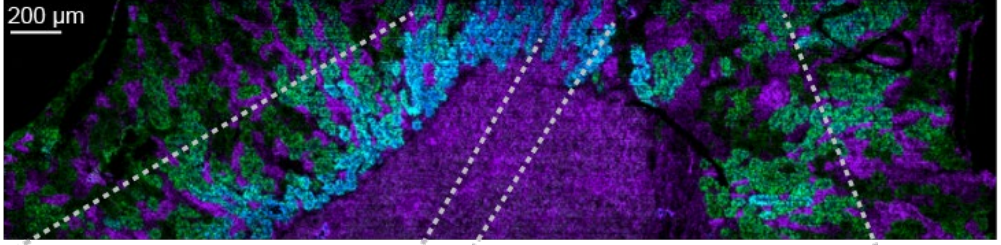

c)

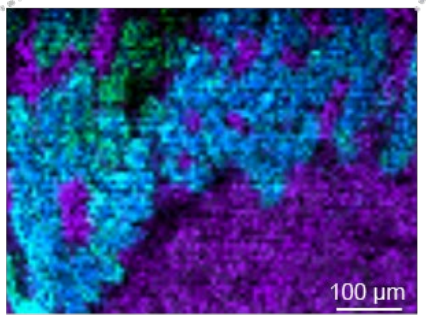

e)

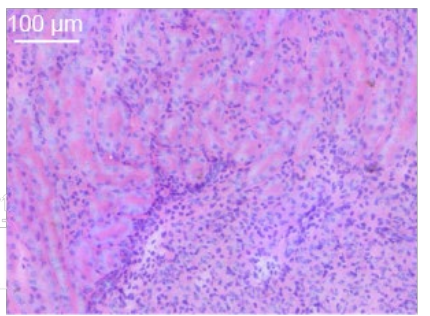

d)

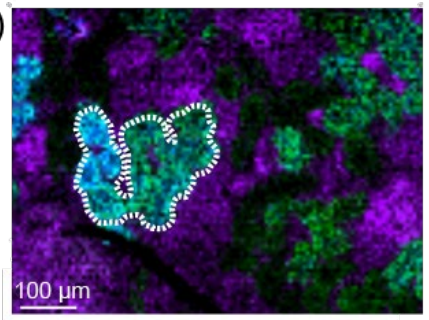

f)

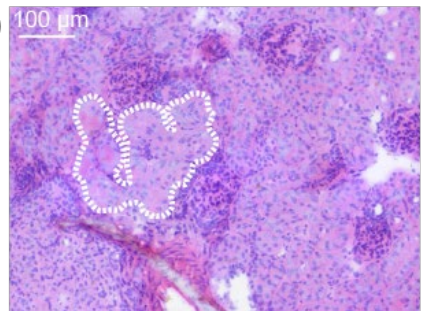

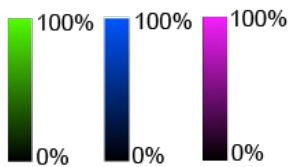

$\mathrm{m} / \mathrm{z} 806.5702$

- $\mathrm{PC}(38: 6)+\mathrm{H}]^{+}$

$$
\begin{aligned}
& m / z \text { 774.5430 } \\
& {[\mathrm{PE}(\mathrm{O}-40: 8)+\mathrm{H}]^{+}} \\
& m / z \text { 724.5287 } \\
& {[\mathrm{PE}(\mathrm{O}-36: 5)+\mathrm{H}]^{+}}
\end{aligned}
$$

Figure 4.9 (a) Optical image of the post-MSI H\&E-stained tissue section. (b) Ion distributions images of $[\mathrm{PC}(38: 6)+\mathrm{H}]^{+}$(green), $[\mathrm{PE}(\mathrm{O}-40: 8)]^{+}$, (blue) and $[\mathrm{PE}(\mathrm{O}-36: 5)+\mathrm{H}]^{+}$(pink) throughout mouse kidney tissue acquired using MALDI-2 and a pixel size of $6 \mu \mathrm{m}$. (c, d) Selected enlarged regions of the MSI data. The corresponding H\&E images of these enlarged regions are shown in (e, f). All MSI data is visualized using total ion current normalization and hotspot removal (99\% quantile). 


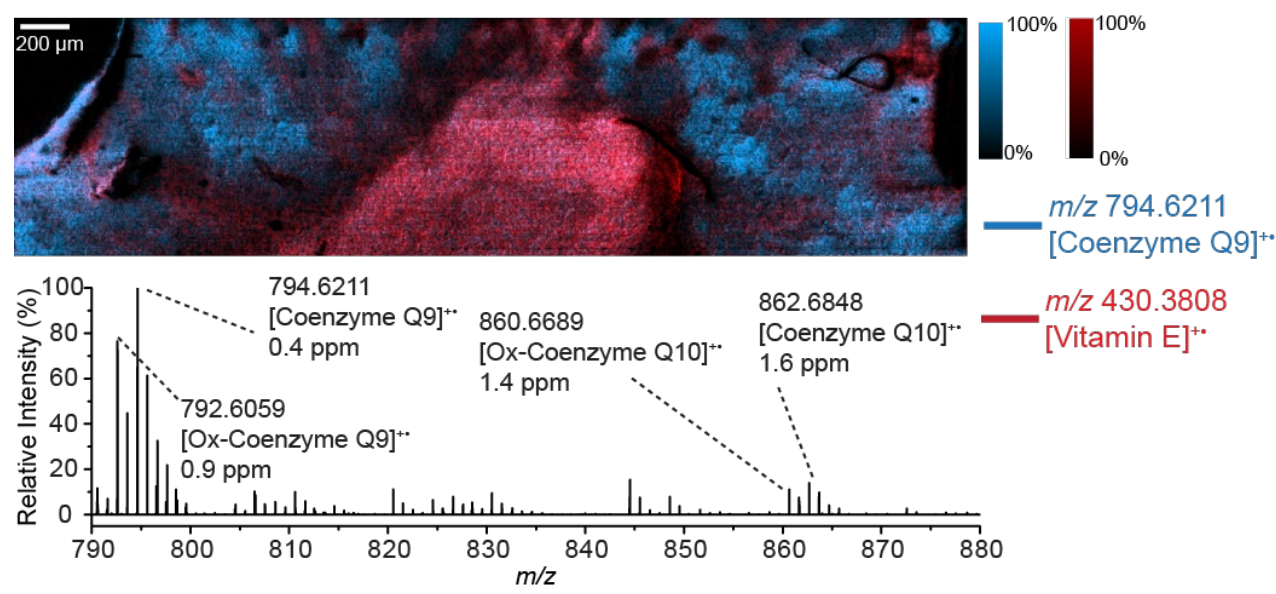

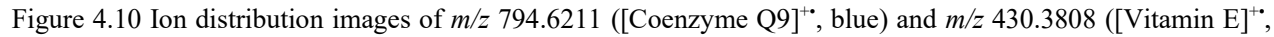
red) throughout mouse kidney tissue. The corresponding MALDI-2 mass spectrum showing the detection of both the oxidized and reduced forms of coenzyme Q9 and coenzyme q10 is shown below.

In addition to the $[\mathrm{M}+\mathrm{H}]^{+}$and $\left[\mathrm{M}+\mathrm{H}-\mathrm{H}_{2} \mathrm{O}\right]^{+}$ions automatically annotated we also observed a variety of radical cations corresponding to different lipidsoluble antioxidants within the mouse kidney tissue. These species are generated by direct absorption of the $260 \mathrm{~nm}$ MALDI-2 laser light via a [1+1] resonance enhanced multiphoton ionization (REMPI) process. In particular, we observed the radical cation of Vitamin $\mathrm{E}$ at $\mathrm{m} / \mathrm{z} 430.3808$ which exhibited elevated signal within the inner medulla (Figure 4.10, red). We also observed both the oxidized and reduced forms of coenzyme Q9 $(\mathrm{m} / \mathrm{z} 792.6059$ and $\mathrm{m} / \mathrm{z} 794.6211)$ and coenzyme Q10 (m/z 860.6689 and $m / z 862.6848)$ as shown in the Figure 4.10 spectrum. These constituted abundant signals with the reduced coenzyme Q9 corresponding to the base peak in the spectrum when using a $6 \mu \mathrm{m}$ step size (Figure 4.8a). MS/MS spectra supporting the identification of these radical species are provided in Figure 4.11 and Figure 4.12. The high signals for these species can possibly be explained a higher efficiency REMPI process that is decoupled from the MALDI-based ionization processes. All four of these ion signals exhibited similar distributions and were observed throughout most of the tissue with the distribution of the reduced form of coenzyme Q9 shown in blue in Figure 4.10. While the protonated forms of oxidized and reduced coenzyme Q9 and Q10 have been detected using MALDIMSI from brain tissue [313], to our knowledge this is the first report of their detection in renal tissue with MSI. 


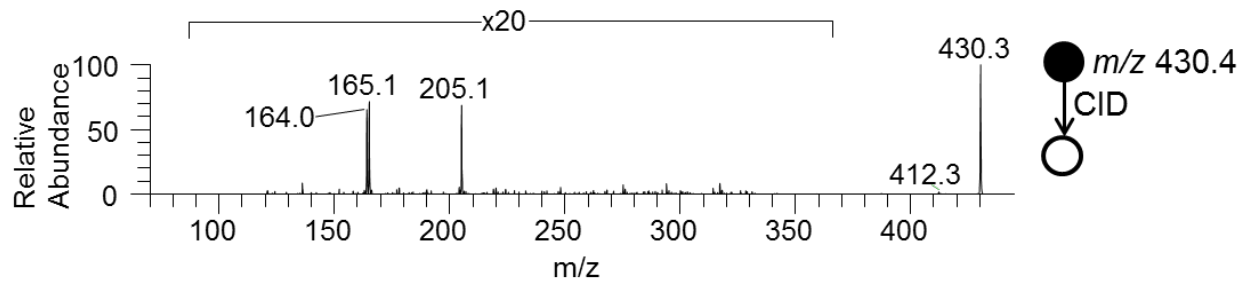

Figure 4.11. Ion trap MS/MS spectrum of $\mathrm{m} / \mathrm{z} 430.4 \pm 0.5$ acquired from mouse kidney tissues using MALDI-2. The MS/MS spectrum confirms the identity of the precursor ion at 430.3808 as the radical cation of Vitamin E. Fragments at m/z 146.0, 164.1 and 205.0 are consistent with those observed in the electron ionization spectrum of the same precursor ion.

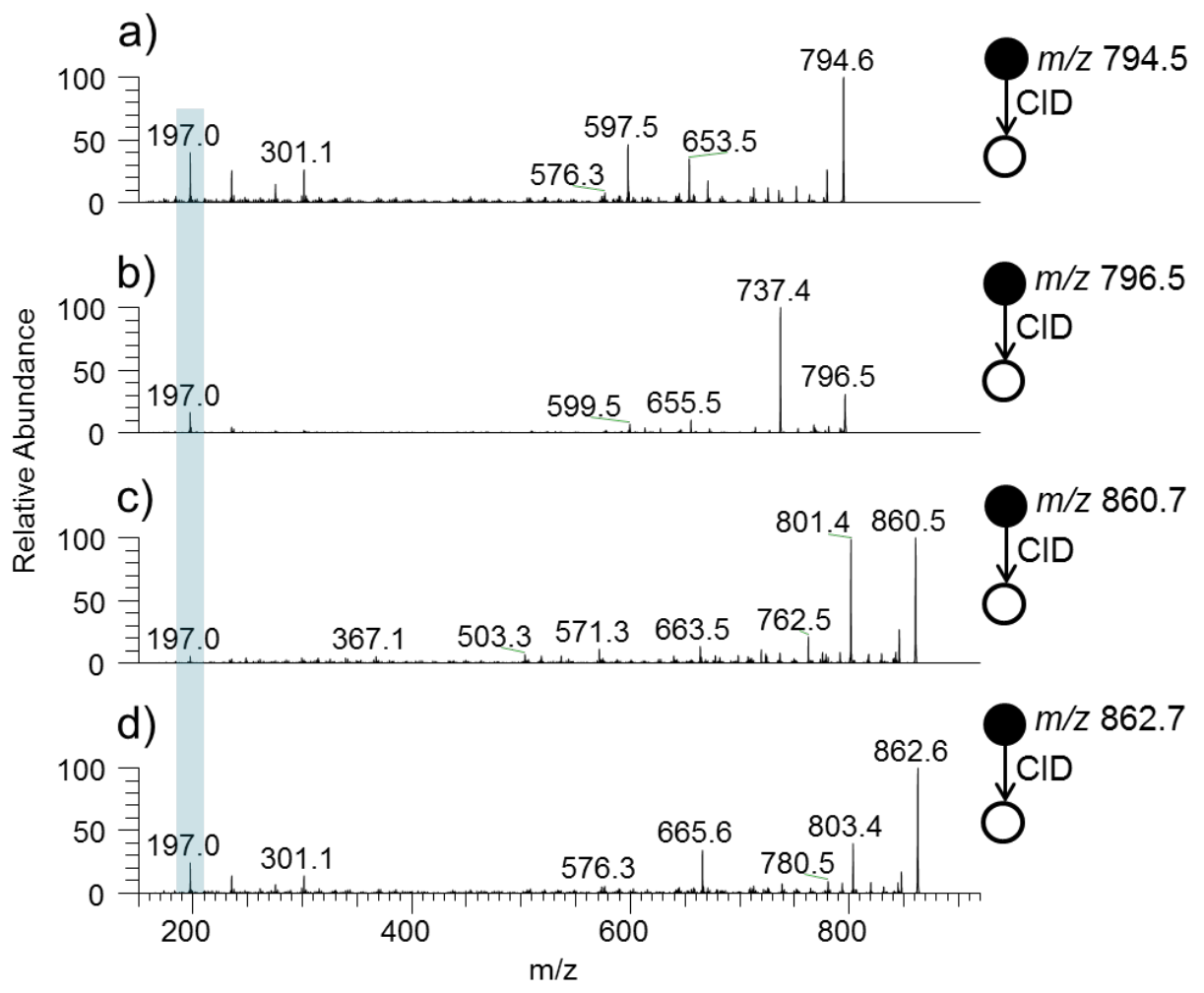

Figure 4.12. Ion trap MS/MS spectrum of (a) $\mathrm{m} / \mathrm{z} 794.5 \pm 0.5$, (b) $\mathrm{m} / \mathrm{z} 796.5 \pm 0.5$, (c) $\mathrm{m} / \mathrm{z} 860.7 \pm 0.5$ and (d) $\mathrm{m} / \mathrm{z}$ $862.7 \pm 0.5$. The MS/MS spectra supports the identification of the precursor ions observed at $\mathrm{m} / \mathrm{z} 792.6059, \mathrm{~m} / \mathrm{z}$ 796.6211, m/z 860.6689 and m/z 862.6848 in Figure 4 as oxidized coenzyme Q9, coenzyme Q9, oxidized coenzyme Q10 and coenzyme Q10, respectively. The detection of a common radical fragment ion is consistent with that reported in the literature [314]. 


\section{SCLEROSIS LESIONS}

Finally, the lipid imaging capabilities of MALDI-2 coupled with oversampling was evaluated using active human multiple sclerosis tissue where the high spatial resolution enabled specific lipid accumulations to be visualized within the tissue lesions. During multiple sclerosis, an autoimmune response is directed against the lipid rich myelin sheath surrounding axons. Myelin is broken down and cleared by phagocytes which causes failure of axonal conduction and, depending on the affected region, disease symptoms such as impaired muscle control, balance, vision, and speech. Myelin processing within the phagocytes leads to the release of lipid mediators that direct the function of the phagocytes and thereby lesion progression and resolution [315-317]. More generally, lipids have been identified to play important roles in multiple sclerosis [318-321], but the precise processes and mechanism by which this occurs, along with the specific functions of individual lipid molecules, remains unknown.

Within the imaging dataset acquired from a $\sim 4.1 \mathrm{~mm}^{2}$ area of tissue and consisting of 114,263 pixels 147 unique sum-composition lipid species were identified (see Methods for identification details). Each of these lipid species revealed tissue-specific distributions throughout the brain tissue. The mean spectrum from this dataset is provided in Figure 4.13a while the distribution of the lipids across the different classes is shown in Figure 4.13b. Again, PC and PE lipids contribute the largest fraction of identified species. Other species with significant contributions to the identified list were sterols, PS and hexosylceramides (HexCer) species. The detection of a number of glycosphingolipids is consistent with their known high abundance and diversity in brain tissue [322]. The full list of identified species is provided in Electronic Supplementary Material Table S3 (not reproduced in this work). 
a) Average Spectrum

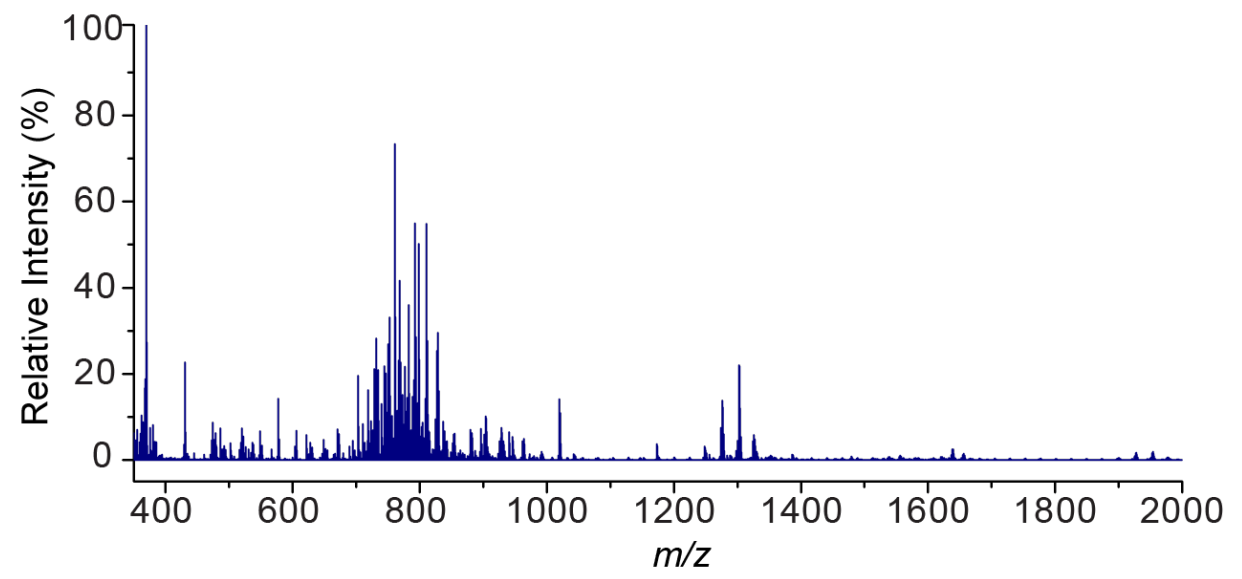

b) Sum-Composition Lipid Identifications from Human Multiple Sclerosis Brain

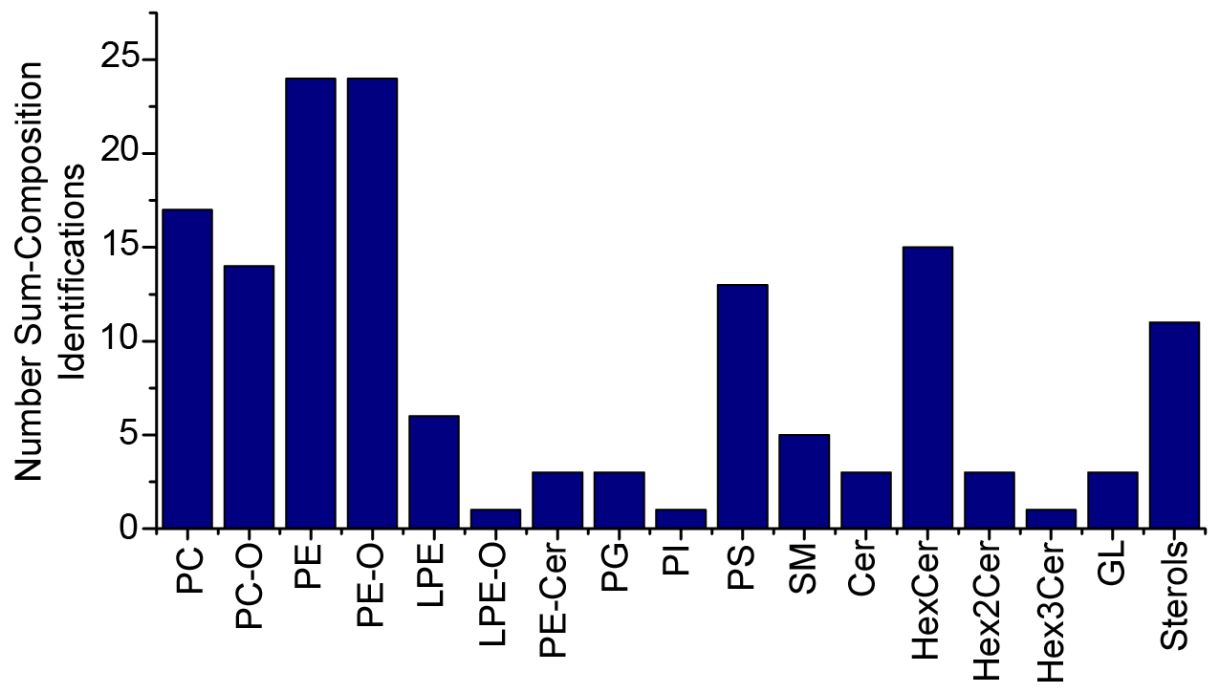

Lipid Class

Figure 4.13 (a) Average spectrum acquired from human multiple sclerosis brain tissue using MALDI-2 and a pixel size of $6 \mu \mathrm{m}$ between $\mathrm{m} / \mathrm{z} 350-2000$. (b) Number of automatically identified lipid species from human multiple sclerosis brain tissue. Lipids were identified as $[\mathrm{M}+\mathrm{H}]^{+}$ions $\left(\left[\mathrm{M}+\mathrm{H}-\mathrm{H}_{2} \mathrm{O}\right]^{+}\right.$for sterols and $[\mathrm{M}+\mathrm{K}]^{+}$for cholesterol esters) using an $\mathrm{m} / \mathrm{z}$ tolerance of $2 \mathrm{ppm}$.

MSI of the human brain tissue using a $6 \mu \mathrm{m}$ pixel size yielded both high contrast and spatially specific signals for many lipid species, thereby allowing visualization of fine structural features only $10-20 \mu \mathrm{m}$ in size. Histopathological analysis was performed on a multiple sclerosis lesion acquired from the same patient revealing this lesion to contain abundant macrophages and microglia (CD68 
staining, Figure 4.14a). These macrophages show a foamy appearance and are filled with esterified cholesterol and other neutral lipids as demonstrated with a Bodipy (Figure 4.14a) and Oil-red O staining (ORO, Figure 4.14b) staining. The high single pixel spectral quality and rich lipid signals achieved from only $6 \mu \mathrm{m}$ pixels is demonstrated in Figures 6e-g that show the corresponding spectra obtained from the individual pixels indicated by the white arrows in Figure 4.14c. The overlaid distributions of three lipid ions, $\left[\mathrm{Chol}+\mathrm{H}-\mathrm{H}_{2} \mathrm{O}\right]^{+}$(pink), $[\mathrm{HexCer}(\mathrm{d} 36: 2)+\mathrm{H}]^{+}$(blue) and the cholesterol ester $(\mathrm{CE})[\mathrm{CE}(16: 0)+\mathrm{K}]^{+}$(green), is shown in Figure $4.14 \mathrm{c}$ and clearly correlate with distinct tissue regions. Two enlarged regions of the MSI data are shown in Figure 4.14d and e to further highlight these specific distributions. 

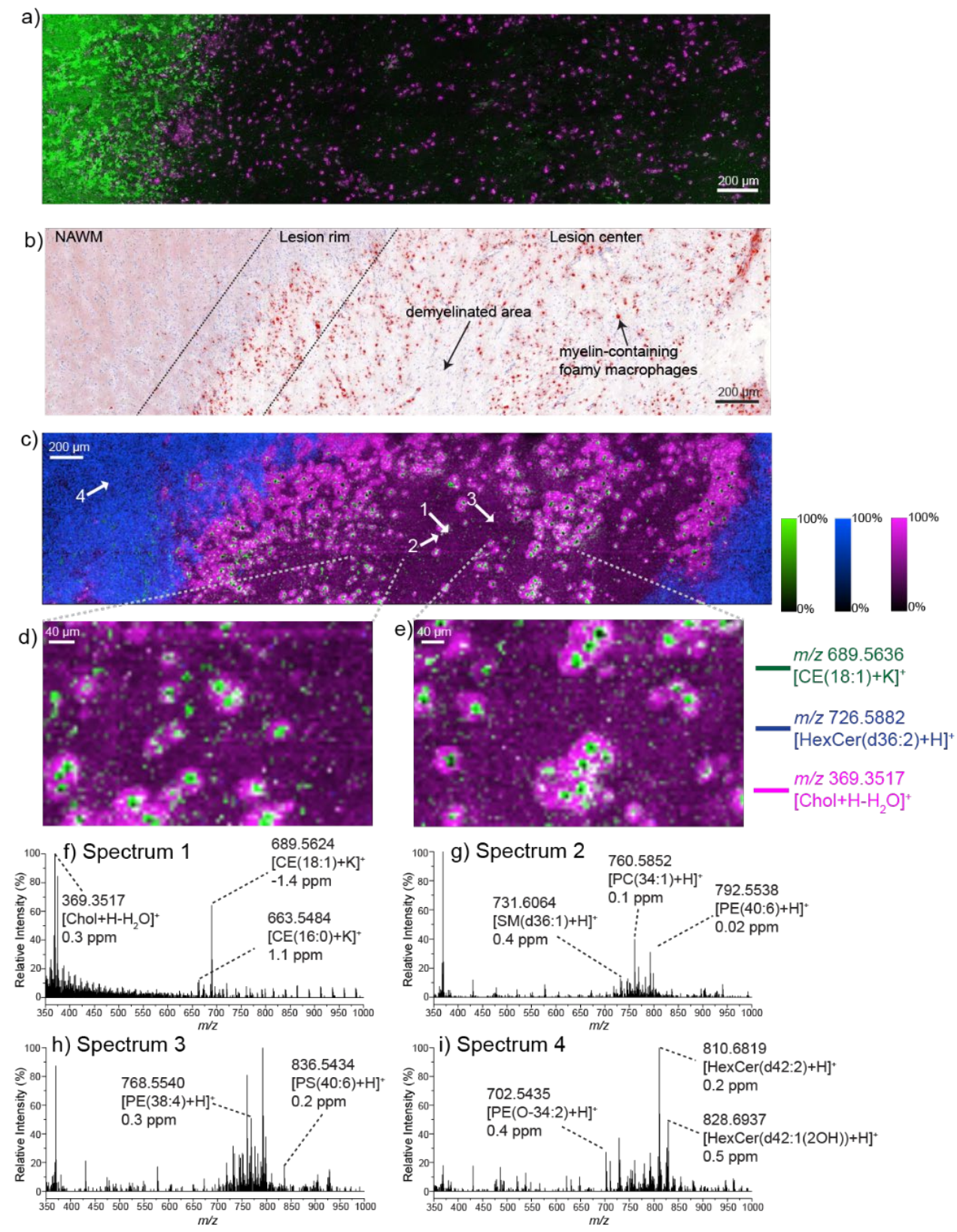

Figure 4.14 (a) CD68 (macrophages, purple) and Bodipy (myelin/neutral lipids, green) immunostaining and (b) Oil Red O staining of human brain tissues slices acquired from the same patient used to collect the MSI data shown in (c-e) with several tissue regions indicated. (c) Ion distributions images of $m / z 689.5636$ ([CE(18:1)+K] $]^{+}$, green), $m / z 726.5882\left([\mathrm{HexCer}(\mathrm{d} 36: 2)+\mathrm{H}]^{+}\right.$, blue) and $m / z 369.3517\left(\left[\mathrm{Chol}+\mathrm{H}-\mathrm{H}_{2} \mathrm{O}\right]^{+}\right.$, pink) acquired using MALDI-2 and a pixel size of $6 \mu \mathrm{m}$ from human multiple sclerosis brain tissue. (d, e) Selected enlarged regions of the MSI data shown in (c). (f-i) Single pixel spectra acquired from the regions indicated by the white arrows in (c). All MSI data is visualized using the total ion current normalization and hotspot removal ( $99 \%$ quantile). NAWM $=$ normal appearing white matter. 
Analogous distributions were also observed in brain tissue acquired from a second multiple sclerosis patient (Figure 4.15). Signal for [HexCer(d36:2)] ${ }^{+}$was localized to the myelin surrounding the multiple sclerosis lesion and was virtually absent within the lesion. Signal for the CE species, $[\mathrm{CE}(18: 1)+\mathrm{K}]^{+}$is localized in areas only 10-20 $\mu \mathrm{m}$ wide and corresponded to the lipid accumulations within the phagocytes as shown in the ORO staining (Figure 4.14a, b).

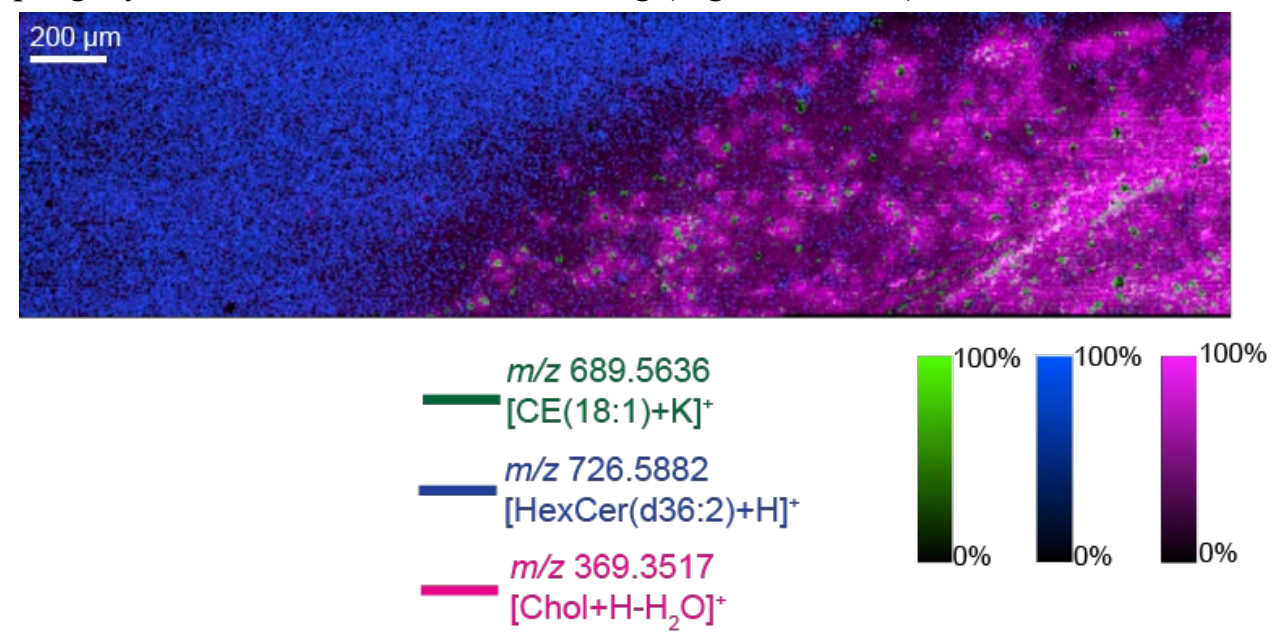

Figure 4.15. MALDI-2 MSI data of human brain tissue contacting active multiple sclerosis lesions that was obtained from a different patient compared to that shown in Figure 6. Data was acquired using a $6 \mu \mathrm{m}$ step size and shows the distribution of $\mathrm{m} / \mathrm{z} 689.5636\left([\mathrm{CE}(18: 1)+\mathrm{K}]^{+}\right.$, green $), m / z 726.5682\left([\mathrm{HexCer}(\mathrm{d} 36: 2)+\mathrm{H}]^{+}\right.$, blue) and $\left[\mathrm{Chol}+\mathrm{H}-\mathrm{H}_{2} \mathrm{O}\right]^{+}$, pink). Scale bar is $200 \mu \mathrm{m}$.

A similar distribution is also observed for the potassium adduct of $\mathrm{CE}(16: 0)$ (Figure 4.16). Interestingly signal for $\left[\mathrm{Chol}+\mathrm{H}-\mathrm{H}_{2} \mathrm{O}\right]^{+}$, likely representing both free cholesterol and in-source fragmentation of CEs, localized to a wider region adjacent to the centers of the phagocyte lipid accumulations. Also, abundant higher $\mathrm{m} / \mathrm{z}$ signals observed at $\mathrm{m} / \mathrm{z} 1302.2069$ and $\mathrm{m} / \mathrm{z} 1276.1906$ assigned based on accurate mass to the protonated $\mathrm{CE}$ dimer species $\left([2 \mathrm{CE}(18: 1)+\mathrm{H}]^{+}(-0.3\right.$ ppm error) and $[\mathrm{CE}(18: 1)+\mathrm{CE}(16: 0)+\mathrm{H}]^{+}(-1.0 \mathrm{ppm}$ error $)$ yielded similar distributions as observed for $\left[\mathrm{Chol}+\mathrm{H}-\mathrm{H}_{2} \mathrm{O}\right]^{+}$(Figure $4.16 \mathrm{~b}$ and c). Evidence for the formation of $[2 \mathrm{M}+\mathrm{H}]^{+}$ions of $\mathrm{CE}$ was obtained upon analyzing a $\mathrm{CE}(18: 0)$ standard using MALDI-2 that yielded an analogous $[2 \mathrm{CE}(18: 0)+\mathrm{H}]^{+}$dimer (Figure 4.17). Despite the CE-related signals in the regions around the phagocytes there is little signal in the ORO staining within these regions. ORO staining widely thought 
to be specific for neutral lipid droplets (e.g., those containing $\mathrm{CE}$ and triacylglyceride lipids) and does not strongly stain other cellular/tissue regions outside these regions that contain other lipid species. We therefore speculate that the lack of ORO staining in the regions adjacent to the phagocytes is caused by the presence of other lipid species. For example, two PC-O species, $\left([\mathrm{PC}(\mathrm{O}-40: 7)+\mathrm{H}]^{+}\right.$ and $[\mathrm{PC}(\mathrm{O}-40: 6)+\mathrm{H}]^{+}$also found specifically in the regions adjacent to the phagocytes (Figure 4.16).

We also note that the mechanism leading to alkali adducted CE species being observed in the phagocytes and protonated-related signals surrounding these regions is unknown. These results suggest specific functions of both free cholesterol and CEs in multiple sclerosis, and in particular phagocyte metabolism. Accumulation of cholesterol in macrophages activates the nuclear liver-Xreceptors that modulate cellular lipid metabolism and the production of inflammatory mediators [316]. Interestingly, aging was shown to promote cholesterol accumulation in phagocytes which stimulates the inflammatory activity of these immune cells and impairs their reparative properties [323, 324]. More generally, the localization of certain lipid species to phagocytes within the brain tissue confirms the high, cellular-level, spatial resolution that can be achieved with this method, while still obtaining rich lipid spectra. This broad lipid coverage and high spatial resolution enabled MALDI-2 coupled with oversampling can provide a new tool to study in depth the lipid-specific alterations of different cell populations within multiple sclerosis progression as well as other neurodegenerative diseases. 
(a) $m / z 663.5484-[\mathrm{CE}(16: 0)+\mathrm{K}]^{+}(1.1 \mathrm{ppm})$

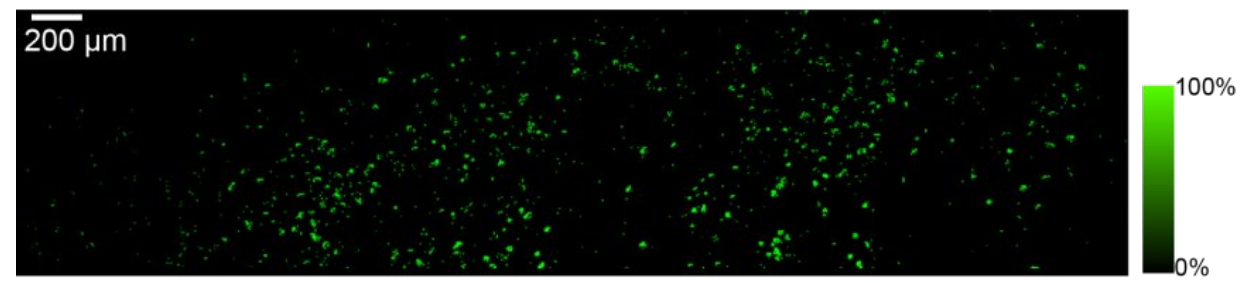

(b) $m / z 1302.2069-[2 \mathrm{CE}(18: 1)+\mathrm{H}]^{+}(-1.0 \mathrm{ppm})$

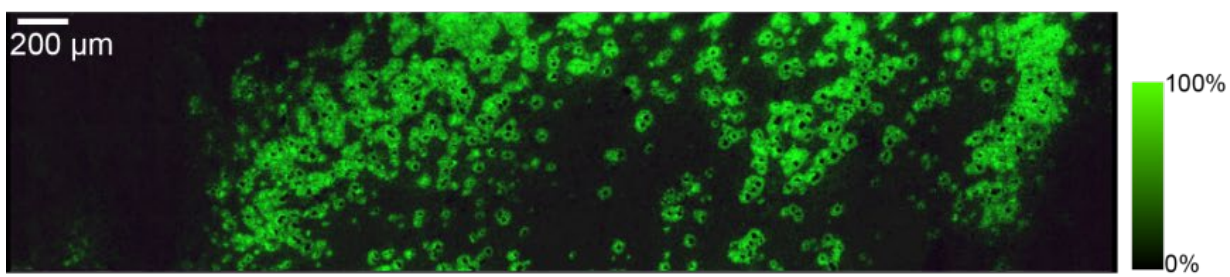

(c) $m / z$ 1276.1906 - [CE(18:1)+CE(16:0)+H] $]^{+}(-0.3 \mathrm{ppm})$

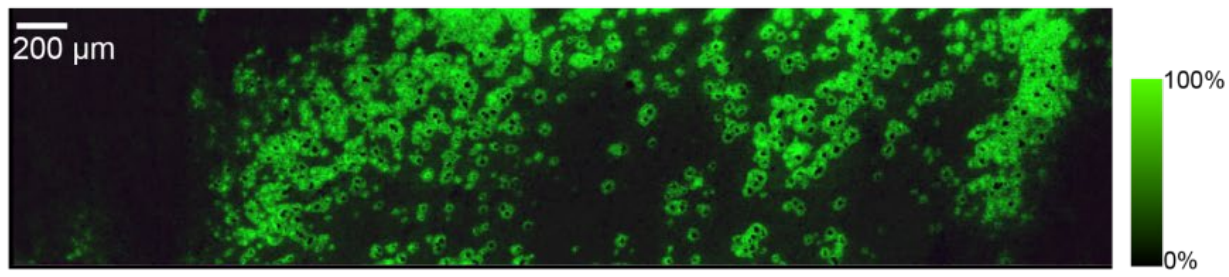

(d) $\mathrm{m} / \mathrm{z} 820.6214-[\mathrm{PC}(\mathrm{O}-40: 6)+\mathrm{H}]^{+}(-0.08 \mathrm{ppm})$

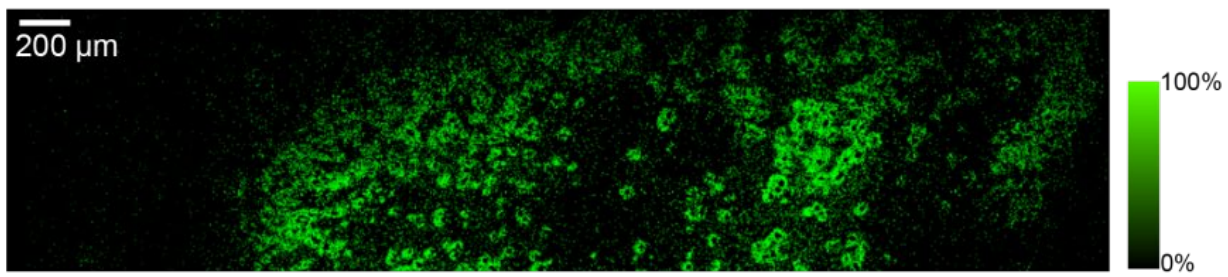

(e) $\mathrm{m} / \mathrm{z} 818.6058-[\mathrm{PC}(\mathrm{O}-40: 7)+\mathrm{H}]^{+}(-0.02 \mathrm{ppm})$

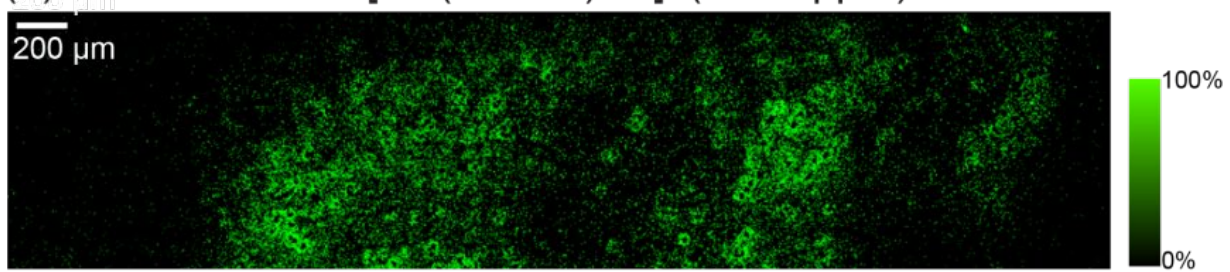

Figure 4.16. MALDI-2-MSI ion distribution images acquired from human multiple sclerosis brain tissue using a $6 \mu \mathrm{m}$ pixel size. (a) $\mathrm{m} / \mathrm{z} 663.5484([\mathrm{CE}(16: 0)+\mathrm{K}]+$, (b) $\mathrm{m} / \mathrm{z} 1302.2069([2 \mathrm{CE}(18: 1)+\mathrm{H}]+$, (c) $\mathrm{m} / \mathrm{z} 1276.1906$ $\left([\mathrm{CE}(18: 1)+\mathrm{CE}(16: 0)+\mathrm{H}]+\right.$, , (d) $\mathrm{m} / \mathrm{z} 820.6214\left([\mathrm{PC}(\mathrm{O}-40: 6)+\mathrm{H}]^{+}\right.$and (e) $m / z 818.6058\left([\mathrm{PC}(\mathrm{O}-40: 7)+\mathrm{H}]^{+}\right.$. All images are visualized using total ion current normalization and hotspot removal $99 \%$ quantile). 


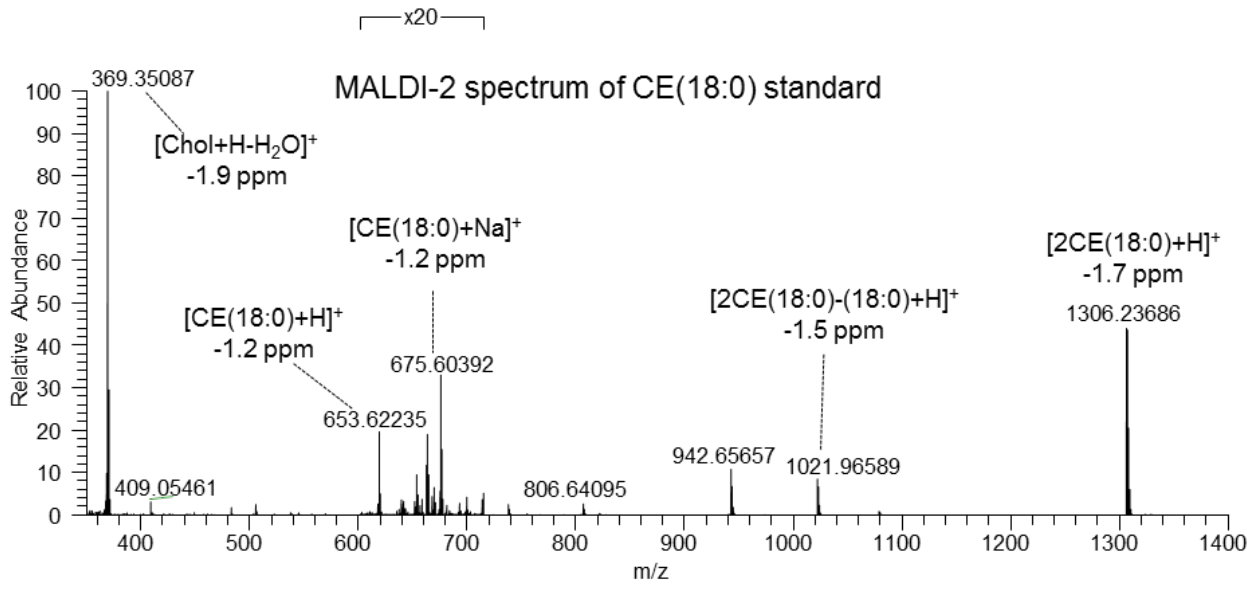

Figure 4.17. MALDI-2 spectrum of a $\mathrm{CE}(18: 0)$ standard demonstrating the formation of abundant $[2 \mathrm{M}+\mathrm{H}]+$ ions $(\mathrm{m} / \mathrm{z}$ 1306.23686) as well as other CE(18:0)-related ions. A $1 \mu \mathrm{L}$ aliquot of a $2 \mathrm{mM} \mathrm{CE}(18: 0)$ standard was spotted onto an ITO slide. Once dried the ITO slide was sublimed with DHB and recrystallized using identical parameters as sued to prepare the human brain tissue.

\section{CONCLUSIONS}

In this work we have demonstrated that MALDI-2 combined with an oversampling acquisition approach is capable of both generating rich lipid spectra from tissue and imaging lipids at pixel sizes as low as $6 \mu \mathrm{m}$, without modifications to the optics or stage of the commercially available ion source. MALDI-2 data generated from $6 \mu \mathrm{m}$ pixels was capable of detecting three times more lipid species than conventional MALDI acquired without oversampling at $20 \mu \mathrm{m}$ pixel size. From kidney and brain tissue imaging experiments we could identify 74 and 147 unique lipid species, in addition to visualizing their spatial distributions within the tissue. Importantly lipid spectra obtained from individual pixels represent an area equivalent to or less than the size of a typical mammalian cell. This is an important capability that will greatly enhance the ability to interpret lipid MSI data in terms of cellular-level lipid metabolism occurring within biological tissues, while preserving the context of the cell within the tissue microenvironment. For example, data acquired from mouse kidney clearly enables the localization of lipid signals to individual tubuli, while data from human multiple sclerosis tissue enables lipid accumulations within lesion-specific macrophages to be visualized. The combination of information rich spectra and cellular-level spatial resolutions 
provides a powerful approach to study spatial and cell-type specific alterations in lipid metabolism within many different disease types.

From a technology standpoint, this work provides the most comprehensive overview of the lipid detection capabilities of MALDI-2 reported to-date, demonstrating the detection of many more lipid classes than possible with positiveion mode MALDI. Moreover, the coupling of this approach with a new automated lipid identification workflow utilizing accurate $\mathrm{m} / \mathrm{z}$ measurements enables the rich lipidomics information acquired in such experiments to be readily exploited. 


\section{Chapter 5 High THROUGHPUT AND}

HigH SENSITIVITY ISOMERRESOLVED IMAGING OF LIPIDS BY INTEGRATION OF OZONE-INDUCED DISSOCIATION WITH A MALDI-
QTOF MASS SPECTROMETER

Britt S.R. Claes ${ }^{1 \#}$, Andrew P. Bowman ${ }^{1 \#}$, Berwyck L.J. Poad ${ }^{2}$, Ron M.A. Heeren ${ }^{1}$, Stephen J. Blanksby ${ }^{2}$ and Shane R. Ellis ${ }^{1,3 *}$

${ }^{1}$ The Maastricht MultiModal Molecular Imaging (M4I) Institute, Division of Imaging Mass Spectrometry (IMS), Maastricht University, 6229 ER Maastricht, The Netherlands

${ }^{2}$ Central Analytical Research Facility, Queensland University of Technology, Brisbane, Queensland 4001, Australia

${ }^{3}$ Mass Spectrometry Imaging Laboratory, School of Chemistry and Molecular Bioscience, University of Wollongong, Wollongong, New South Wales 2522, Australia

\# These authors have equally contributed 


\section{ABSTRACT}

Lipids are a diverse group of biomolecules involved in various biological processes, of which its functionality is highly dependent on the molecular structure. However, while basic MS methods can identify sum compositions, other levels of specificity to identify lipids uniquely, such as the double bond $(\mathrm{db})$ location and the sn-position, are left unidentified. In this study, ozone-induced dissociation (OzID) was integrated with a MALDI-Q-TOF mass spectrometer to exploit the gas-phase reaction between mass-selected lipid ions and ozone vapor. With gas pressures several orders of magnitude higher than can be achieved in a linear ion trap, a 1,000fold enhancement in reaction rates were achieved. Because of the higher sensitivity and faster reaction speed with this method, OzID imaging data were obtained at a 50-fold improvement in acquisition rate compared to before with the ion trap, allowing increasing the analytical detail by using a higher spatial resolution or acquiring bigger tissue areas, or increasing the throughput. Aside from regular OzID to determine the $\mathrm{db}$ position, also CID/OzID experiments to determine the sn-position were performed. As the instrument was limited to MS2 experiments, only the intact lipid could be isolated. This led to both OzID and CID/OzID fragments to be present in the spectrum. The CID/OzID fragments showed to even further oxidize and form CID/OzID2 fragments, which were specific for the $\mathrm{db}$ position at the sn-1 fatty acid. Hence, our method demonstrated to overcome several limitations of lipid structure characterization and could be valuable to gain localized isomeric information.

\section{INTRODUCTION}

Lipids are the primary constituents of cell membranes[41] and are key molecular players in many biochemical functions including energy storage[325, 326], signal transduction[327, 328], and apoptosis[329, 330], as well as cancer metabolism[22, 331, 332]. Crucially, the precise function and biological implication(s) of a particular lipid is highly dependent on its chemical structure. For example, subtle alterations in acyl chain and/or double bond (db) positions can influence the physical chemical properties of lipid membranes[333] and lipidprotein interactions can be specific for a certain isomeric structure[334]. Further highlighting the importance of lipid structure in understanding function is the precise regulation of lipid synthesis, even at the isomer level in different tissues[335, 
336] and the observed alteration of isomer populations in cancer metabolism[22, 337-339] and diabetes[340-344].

Elucidating the composition of tissue and cellular lipidomes has, over the past 2 decades, been spearheaded by continually advancing mass spectrometry (MS) technologies and methodologies[345-347]. The workhorse of modern lipidomics is the analysis of biological lipid extracts via electrospray ionization (ESI) using either shotgun[58, 347-349] or chromatographic[350-354] approaches. When coupled with high mass resolving power and high mass accuracy such methods are capable of identifying many lipid species to the sum-composition level[355]. Oftentimes such methods are combined with conventional low energy collision induced dissociation (CID) that can provide additional structural information such as the length and degree of unsaturation of individual acyl chains (i.e., identification to the molecular lipid species level). However, despite the widespread and ever-growing popularity of these methods they are still far from being capable of resolving many isomeric lipids (e.g., fatty acyl stereonumber ( $s n$-) position, carbon-carbon $\mathrm{db}(\mathrm{C}=\mathrm{C})$ location, and $\mathrm{db}$ geometry on the acyl chains (i.e., cis or trans)) and providing total structural identification of lipids. Considering the possibility of more than 180,000 unique structures[40, 41], and the intimate link between lipid structure and function, the development of such capabilities represent a critical challenge lipidomics must address.

Both methods can be enhanced with the use of MS/MS, with collision induced dissociation (CID) being the most common. CID can identify headgroup and acyl chain composition (i.e. if a lipid is phosphatidylcholine (PC), and further if PC 36:0 is 18:0_18:0 or 16:0_20:0), but identification of chain position on the glycerol backbone is difficult, though not impossible with multi-step fragmentation protocols[356]. Similarly, CID cannot distinguish double bond position or cis/trans isomers. Evolving research has emphasized the importance of isomeric changes in biological function[357-360], and changes to acyl chain length and sn-, along with $\mathrm{db}$ location, degree, and geometry alter cellular function[361-364].

The realization of the need for more structural information and increasing isomeric resolution has led to a rapid growth in the development of ion activation methods capable of resolving lipid isomers, namely those varying in double bond and sn-position. These include Ozone-Induced Dissociation (OzID)[365], Ultraviolet Photodissociation (UVPD)[161], epoxidation[163], and the Paterno- 
Buchi (PB) reaction[164]. These techniques individually can identify db locations, and either include, or can be combined with, CID to target $s n$ - position, as well.

Extraction based techniques are extraordinarily useful for the identification and quantification of lipids. However, typical experiments homogenize whole tissues prior to extraction, which may mask localized changes in lipid composition and metabolism occurring throughout heterogeneous tissues. Recent work by have shown the isomeric resolution possible by combining OzID[366], UVPD[367], $\mathrm{PB}[359,368,369]$, and epoxidation[370] with mass spectrometry imaging (MSI). In general, these techniques involve either the application of a reactive agent to the entire tissue section (PB and epoxidation), or isolation and reaction of a narrow, isolated $\mathrm{m} / \mathrm{z}$ range (OzID and UVPD). For epoxidation, reactive agents like metachloroperoxybenzoic acid have been used[371], as well as low temperature plasmas[372] and electrochemical stimulation[373]. Epoxidations reactions typically take less than a minute, with electrochemical stimulation happening effectively simultaneous with ionization, though conversion efficiency suffers with shorter reaction times (20\% derivatization being common[164]). PB constitutes a wide variety of reactions that produce four-membered rings at $\mathrm{C}=\mathrm{C}$ bonds, with some of the most popular being acetone[359], benzaldehyde[369], and benzophenone[368]. The advantage of $\mathrm{PB}$ is the relatively high reaction conversion efficiency (up to $40 \%$ having been reported in MSI systems[374]), with a slower reaction speed than epoxidation (on the order of several minutes[368, 369]). For complete lipid identification, both epoxidation and $\mathrm{PB}$ reactions are incomplete, as only db positions (irrespective of $s n$-position) are uniquely identified.

Neither UVPD nor OzID require a derivatization step. UVPD in conjunction with MSI has revealed db positional isomers in multiple tissue types, including brain[359, 367], kidney[359, 367], pancreas[367], and lung[164]. Multiple diagnostic fragments of $\mathrm{db}$ positional isomers are produced through UVPD, though overall efficiency is low (less than $1 \%$ for db-specific fragments[375]). In an imaging modality, only fatty acids[375] and phosphatidylcholines[367] have been analyzed through UVPD, though the overall utility of the technique has been described on cardiolipins[376], other glycerophospholipids[161], and sphingolipids[219]. Additionally, CID/UVPD experiments can distinguish $s n$-isomers, though only $\mathrm{db}$ - or $s n$-isomers can be distinguished in a single experiment[161, 219, 367]. Comparatively, the OzID experiments follow two different reaction schemes that have to-date been 
performed only on PCs in MSI applications: expose a trapped population of ions (approximately a $\pm 1 \mathrm{~m} / \mathrm{z}$ range) to ozone, which fragments the ions at the $\mathrm{C}=\mathrm{C}$ (OzID), or CID of a mass-selected alkali adducted PC first, resulting the loss of the phosphocholine headgroup and rearrangement leading to creation new $\mathrm{C}=\mathrm{C} \mathrm{db}$ that reacts with ozone to remove one acyl chain (CID/OzID) and allow assignment of sn-positioning of acyl chains. In the application of OzID to MALDI, a key limitation thus far has been the slow rate of reaction in the experimental setup, partly due to the vacuum requirements of the linear ion trap (around $10^{-5}$ millibar) and partly due to the helium-based design of the ion trap. Replacing He with $\mathrm{O}_{3}$ in $\mathrm{O}_{2}$ lowers trapping efficiency, so a high proportion of $\mathrm{He}$ must be maintained to maintain ion populations that requires dilution of generated ozone in helium. These combine such that reaction times of 10 seconds per pixel are required to produce diagnostic fragments with an abundance of $2.5 \%$ or less the abundance relative of the precursor ion, making experiments long for even relatively small tissue areas[366]. However, CID/OzID has been shown to be several orders of magnitude faster, allowing $s n$ - isomer identification in as little as $250 \mathrm{~ms}$, providing a timeframe that is more compatible with typical MSI experiments[366].

Recently, new approaches were developed to allow more complete lipid structure determination with MSI on biological tissues[377]. Traveling wave-based OzID-MS has shown to be a useful platform for unsaturated lipid isomer analysis[378, 379]. Poad et al.[379] showed a significant improvement in OzID efficiency by adding ozone in the high-pressure IMS cell ( $\sim 3$ mbar). With gas pressures several orders of magnitude higher than can be achieved in a linear ion trap, 1,000-fold enhancement in reaction rates are possible. This was originally demonstrated with LC-OzID-IMS-MS, separating cis/trans isomers through LC prior to OzID in the trapping region of the instrument before final IMS separation[380, 381]. The benefits of this to MSI experiments are manifold. Overall reaction speed increases through two factors: 1) there is no longer a need in this platform to dilute $\mathrm{O}_{3} / \mathrm{O}_{2}$, allowing for higher partial pressures of $\mathrm{O}_{3}$, and 2) pressure within the ion mobility cell is approximately 3 mbar, an increase of 5 orders of magnitude over the ion trap. This results in a high OzID reaction efficiency using $(10-20 \%)$ [380] reaction time that corresponds only to the typically transit times of ions through the ion mobility region where the zone is introduced (tens of milliseconds). There are several potential benefits of this approach for MSI studies: (i) the ability to operate the MS at its native acquisition rate meaning OzID 
reactions do not adversely affect the acquisition time and thereby allowing imaging of either larger areas or similar areas from previous experiments in practical timeframes; (ii) smaller pixels (with less material ablated) are possible, due to the higher OzID efficiency; (iii) detection of low abundance isomers via the same process; and (iv) the ability to generate OzID and CID/OzID fragments simultaneously if ions are activated prior to entering the ion mobility region.

In this work we report for the first time the coupling of MALDI-MSI on a Synapt G2-Si Q-TOF mass spectrometer with OzID for the high throughput differential imaging of isomeric lipids. The significantly elevated reaction rates provide an up to 1000 -fold decrease in reaction time, resulting in a $\sim 50$-fold gain in acquisition speed for the MSI of double bond isomers. In addition, we demonstrate the ability to acquire $\mathrm{CID} / \mathrm{OzID}^{2}$ data for the first time in an MSI experiment that provide the unique capability of assigning acyl chain double bond positions to individual $s n$-positions. This provides a key breakthrough towards the goal of studying the distributions of structurally defined lipids in tissues.

\section{METHODS}

\section{MATERIALS}

2,5-Dihydroxyacetophenone (DHA, 97\% purity), sodium acetate (anhydrous, $>99 \%$ ), and chloroform ( $\geq 99 \%$ ) were purchased from Sigma Aldrich (Zwijndrecht, The Netherlands) and used without further purification. Methanol (ULC-MS grade), water (LC-MS grade), ethanol (LC-MS grade), and xylene (AR grade) were purchased from Biosolve (Valkenswaard, The Netherlands). Hematoxylin and Entellan ${ }^{\circledR}$ were purchased from Merck (Darmstadt, Germany) and eosin Y from J.T. Baker (Center Valley, USA). Indium tin oxide (ITO)-coated glass slides were purchased from Delta Technologies (Loveland, USA). Lipid standards (PC 18:0/18:1n-9 and PC 16:0/18:1n-9) were purchased from Avanti Polar Lipids (Alabaster, USA).

\section{BIOLOGICAL SAMPLES}

Rats, housed and cared for at the Central Animal Facility of Maastricht University according to local standards, were provided ad libitum access to water and regular chow. Healthy rat brain was obtained in accordance with protocols approved by the Animal Care and Use Committee (DEC number 2014-120). Horizontal sections measuring $10 \mu \mathrm{m}$ thick were prepared using a cryo-microtome 
(Leica, Nussloch, Germany) at $-20^{\circ} \mathrm{C}$, thaw-mounted on ITO-coated glass slides, and stored at $-80^{\circ} \mathrm{C}$ until matrix application and MSI analysis.

SAMPLE PREPARATION

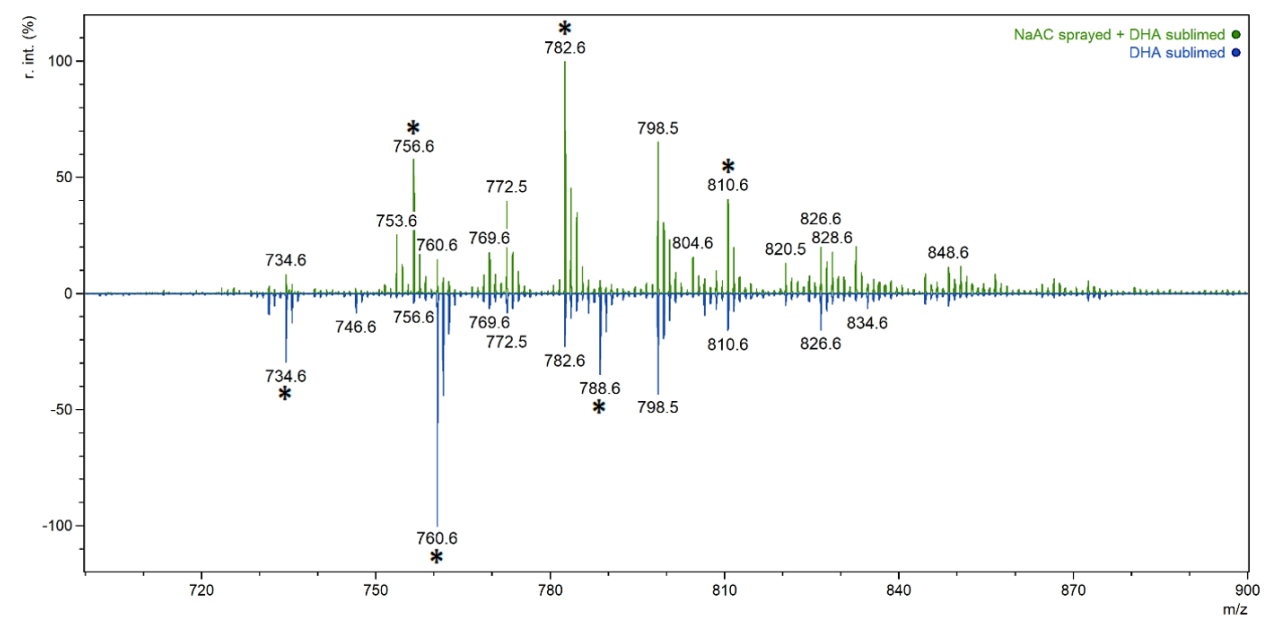

Figure 5.1. Comparison of positive ion MALDI mass spectrum with (top, green) and without (bottom, blue) the sodium acetate application (100 scans averaged). The $\Delta 22$ Da shows the lipids ionized as $[\mathrm{M}+\mathrm{H}]^{+}$ions (in blue) are more prevalent as $[\mathrm{M}+\mathrm{Na}]^{+}$ions (in green) with the sodium acetate sprayed before matrix application. For example, PC 32:0 $[\mathrm{M}+\mathrm{H}]^{+}$at $m / z$ 734.6, $[\mathrm{M}+\mathrm{Na}]^{+}$at $\mathrm{m} / \mathrm{z} 756.6$; $\mathrm{PC} 34: 1[\mathrm{M}+\mathrm{H}]^{+}$at $m / z 760.6,[\mathrm{M}+\mathrm{Na}]^{+}$at $m / z$ 782.6; and PC 36:1 $[\mathrm{M}+\mathrm{H}]^{+}$at $m / z$ 788.6, $[\mathrm{M}+\mathrm{Na}]^{+}$at $m / z$ 810.6.

An automated TM-Sprayer (HTX Technologies, LLC, Chapel Hill, USA) was used for sodium acetate deposition. First, 15 layers of sodium acetate solution (4 mg/mL in 2:1 $\mathrm{CHCl}_{3}: \mathrm{MeOH}(\mathrm{v} / \mathrm{v})$ ) were deposited at $0.12 \mathrm{~mL} / \mathrm{min}, 30 \mathrm{~s}$ drying time between layers, at $30^{\circ} \mathrm{C}$, preferentially forming sodiated ions (Figure 5.1).

Lipid standards were prepared at 0.01 and $0.1 \mathrm{~g} / \mathrm{L}$ concentrations in 2:1 $\mathrm{CHCl}_{3}: \mathrm{MeOH}$ and sprayed on clean ITO slides for 1 to 10 layers following the same protocol as for sodium acetate deposition, creating concentrations from 0.33 to $3.3 \mathrm{ng} / \mathrm{mm}^{2}$ (assuming equal dispersion). Samples were then treated equivalently to brain tissue sections.

Hematoxylin and eosin (H\&E) staining was performed after MALDI imaging. Matrix was removed from tissue by immersion in 70\% ethanol for $3 \mathrm{~min}$. A standard $\mathrm{H} \& \mathrm{E}$ protocol was then used. High-resolution optical images of stained tissues were generated using an Aperio CS2 digital pathology slide scanner (Leica Biosystems, Wetzlar, Germany). 


\section{IN-LINE OZONE GENERATION}

Ozone generation and delivery to the instrument are as described previously.[380] Ozone was produced using a high-concentration ozone generator (TG-40; Ozone Solutions, Hull, IA, USA) from UHP oxygen (5.0 grade, 20 psi @ $0.4 \mathrm{slm}$; Linde Gas Therapeutics Benelux bv, Eindhoven, The Netherlands). Ozone production was optimized to $\sim 275 \mathrm{~g} / \mathrm{Nm} 3$ in $\mathrm{O} 2$ as measured by an in-line monitor (106-H; 2B Technologies, Boulder, USA). The generated ozone/oxygen mixture was connected via a needle valve (SS-SS8; Swagelok, USA) to the ion mobility cell gas inlet of the mass spectrometer and the flow adjusted to maintain a pressure of 2.9 mbar in the cell. Excess ozone was converted to oxygen using an unheated destruct catalyst (810-0008; In USA, Inc., Norwood, USA). Laboratory ambient ozone concentration was monitored (106-L; 2B Technologies, Boulder, USA) and interlocked to shut off the generator if the background ozone level rose above 75 ppb. An instrument schematic is shown in Figure 5.2. 

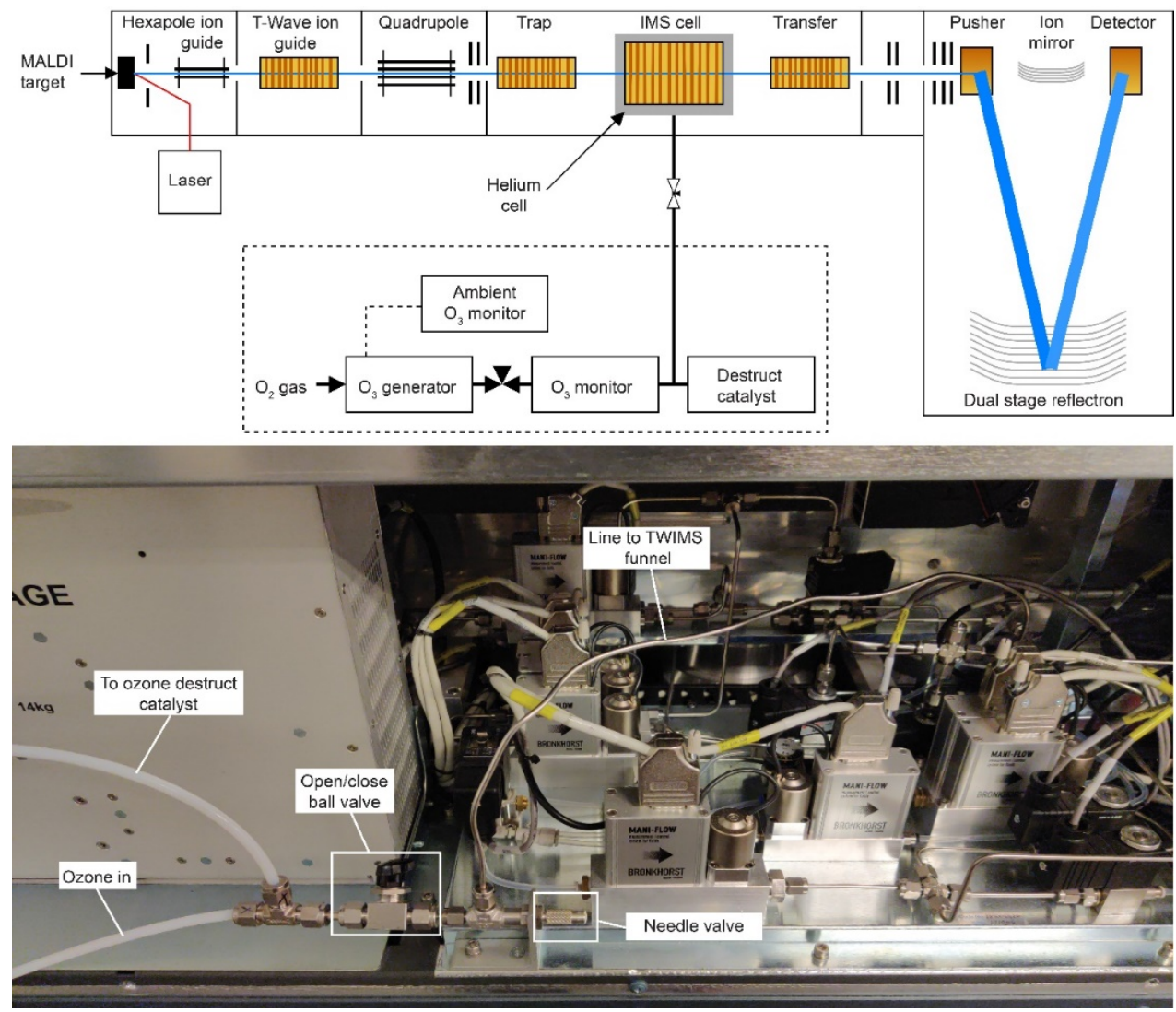

Figure 5.2 Schematic (top) and photograph (bottom) of the modified uMALDI-Synapt-G2-Si system. Ozone was generated by a high-concentration ozone generator and introduced into the TWIMS gas line. The ambient $\mathrm{O}_{3}$ monitor is interlocked to shut down the ozone generator if the background level of ozone in the laboratory exceeds $75 \mathrm{ppb}$.

\section{INSTRUMENTATION}

Tissue sections were analyzed using a prototype $\mu$ MALDI source mounted to a Waters SYNAPT HDMS G2-Si system (Waters Corporation, Manchester, UK), as previously described by Barré et al.[382] Samples were analyzed in continuous raster mode using Waters Research Enabled Software (WRENS) to operate at 5 pixels/s, laser repetition rate of $1500 \mathrm{~Hz}$, pixel size set to $50 \mu \mathrm{m}$, MS/MS isolation window set to $\pm 1.5 \mathrm{~m} / \mathrm{z}$, and detection set to sensitivity mode (mass resolution: $\sim 15,000)$. The laser spot size was approximately $15 \times 15 \mu \mathrm{m}$. Operation of the TWave was optimized to generate the highest intensity of selected diagnostic fragments without depleting the precursor signal (wave velocity $1200 \mathrm{~m} / \mathrm{s}$ and wave 
height $38 \mathrm{~V}$ ). Various traveling wave height and wave velocity settings were tested (Figure 5.3) to find optimal parameters for the OzID reaction in the IMS cell.

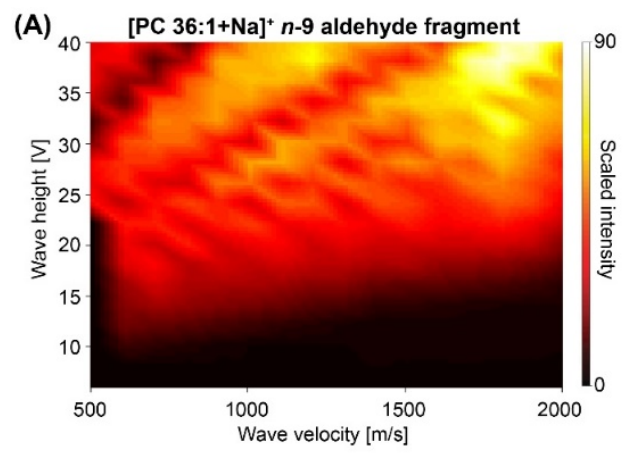

(C)

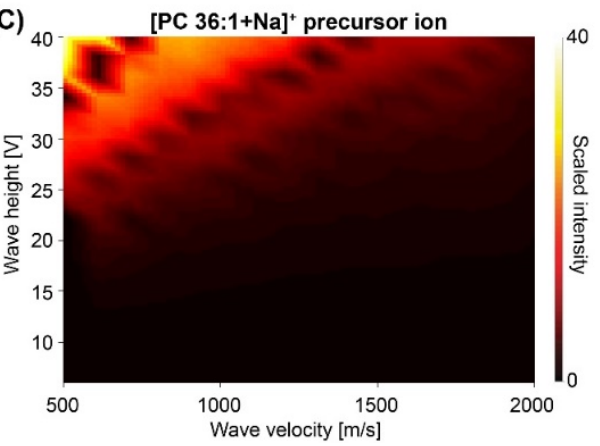

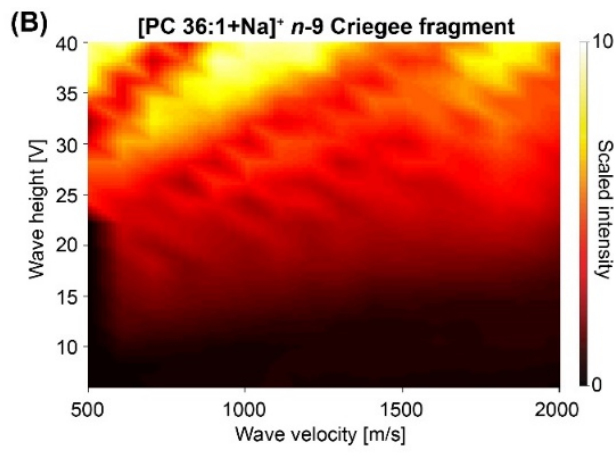

(D) ${ }_{40}$ Sum of [PC $\left.36: 1+\mathrm{Na}\right]^{+}$and $n-9$ OzID fragments

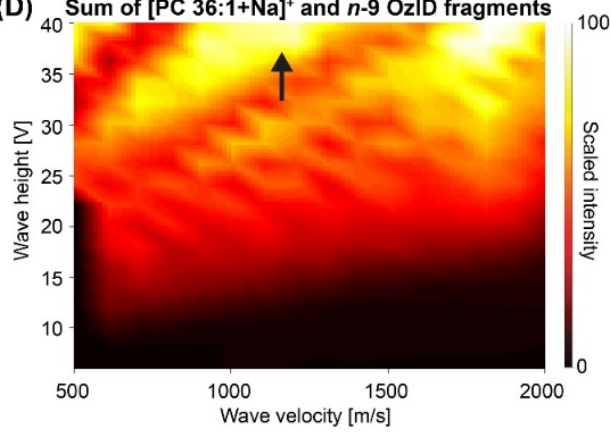

Figure 5.3. Optimization of IMS parameters for the OzID fragments of [PC 36:1+Na $]^{+}$to find the optimum settings for the OzID fragments. The wave height was tested from 6 to $40 \mathrm{~V}$ in $2 \mathrm{~V}$ increments, and the wave velocity was tested from $500-2000 \mathrm{~m} / \mathrm{s}$ in $100 \mathrm{~m} / \mathrm{s}$ increments. (A) Surface plot of the abundance of the $n-9$ aldehyde product ion $(m / z \text { 700.4) arising from OzID of [PC 36:1+Na] }]^{+}$. (B) Surface plot of the [PC $\left.36: 1+\mathrm{Na}\right]^{+} n-9$ Criegee product ion $(\mathrm{m} / \mathrm{z} \text { 716.4). (C) Surface plot of the [PC } 36: 1+\mathrm{Na}]^{+}$precursor ion $(\mathrm{m} / \mathrm{z} 810.6)$. (D) Surface plot of the [PC $36: 1+\mathrm{Na}]^{+}$precursor ion and both the $n-9$ aldehyde and Criegee fragment summed. A wave velocity of $1200 \mathrm{~m} / \mathrm{s}$ and a wave height of $38 \mathrm{~V}$ were chosen as optimum settings as pointed out by the arrow, since there was a higher intensity of the $n-9$ Criegee fragment and the $[\mathrm{PC} 36: 1+\mathrm{Na}]^{+}$precursor ion. The plots display intensity scaled to the highest sum of all three ions.

High traveling wave heights increased apparent fragmentation, while high wave velocities increase ion transmission and fragmentation. Sufficiently high wave height and wave velocity deplete the precursor population. The intensity of two OzID fragments of [PC 36:1+Na] ${ }^{+}(\mathrm{m} / \mathrm{z} 810.6$, Figure 5.3c) were monitored for these experiments: the aldehyde ion at $m / z 700.4$ (Figure 5.3a) and the Criegee ion at $m / z 716.4$ (Figure 5.3b) indicative of the $n-9 \mathrm{db}$ position. Wave height of $38 \mathrm{~V}$ and wave velocity of $1200 \mathrm{~m} / \mathrm{s}$ maximized fragment ion abundance, without eliminating the precursor signal (Figure 5.3d). 


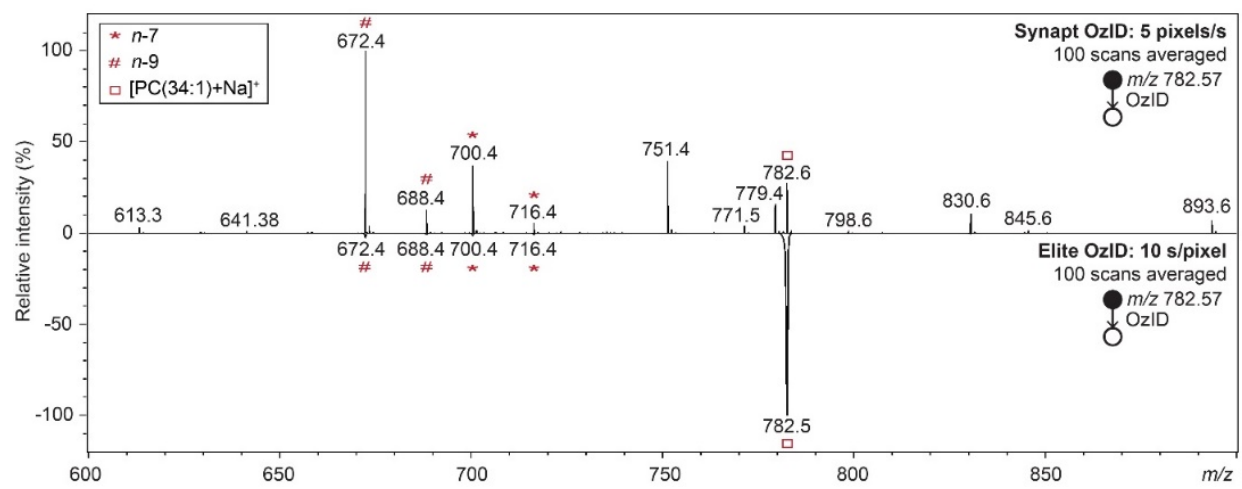

Figure 5.4 Comparison of MALDI-OzID mass spectra of [PC $34: 1+\mathrm{Na}]^{+}$acquired from rat brain tissue in the SYNAPT (top) and the LTQ Orbitrap Elite (bottom). OzID product ions arising from oxidative cleavage of the $n$ $7(\mathrm{~m} / \mathrm{z} 700.4$ and 716.4$)$ and $n-9(\mathrm{~m} / \mathrm{z} 672.4$ and 688.4) db-positions are denoted, together with the precursor [PC $34: 1+\mathrm{Na}]^{+}$at $\mathrm{m} / \mathrm{z}$ 782.6. Synapt OzID product ion yields were 50- to 100 -fold higher with interaction times 50 times lower $(0.01 v s 10 \mathrm{~s})^{1}$ as measured relative to the $[\mathrm{PC} 34: 1+\mathrm{Na}]^{+}$signal $(5 \mathrm{pixels} / \mathrm{s} v s 0.1$ pixels/s). Each spectrum represents the average of 100 scans.

Optimization of the ion mobility traveling wave parameters increased the OzID efficiency, allowing the acquisition speed to be increased 50 times compared to earlier OzID implementations (Figure 5.4) with sensitivity as low as $2.2 \mathrm{fg} / \mu \mathrm{m}^{2}$ (Figure 5.5). The trap and transfer collision energies were set at $4.0 \mathrm{~V}$ and $2.0 \mathrm{~V}$, respectively, for normal OzID, and trap energy was set to $40 \mathrm{~V}$ during CID/OzID experiments. 


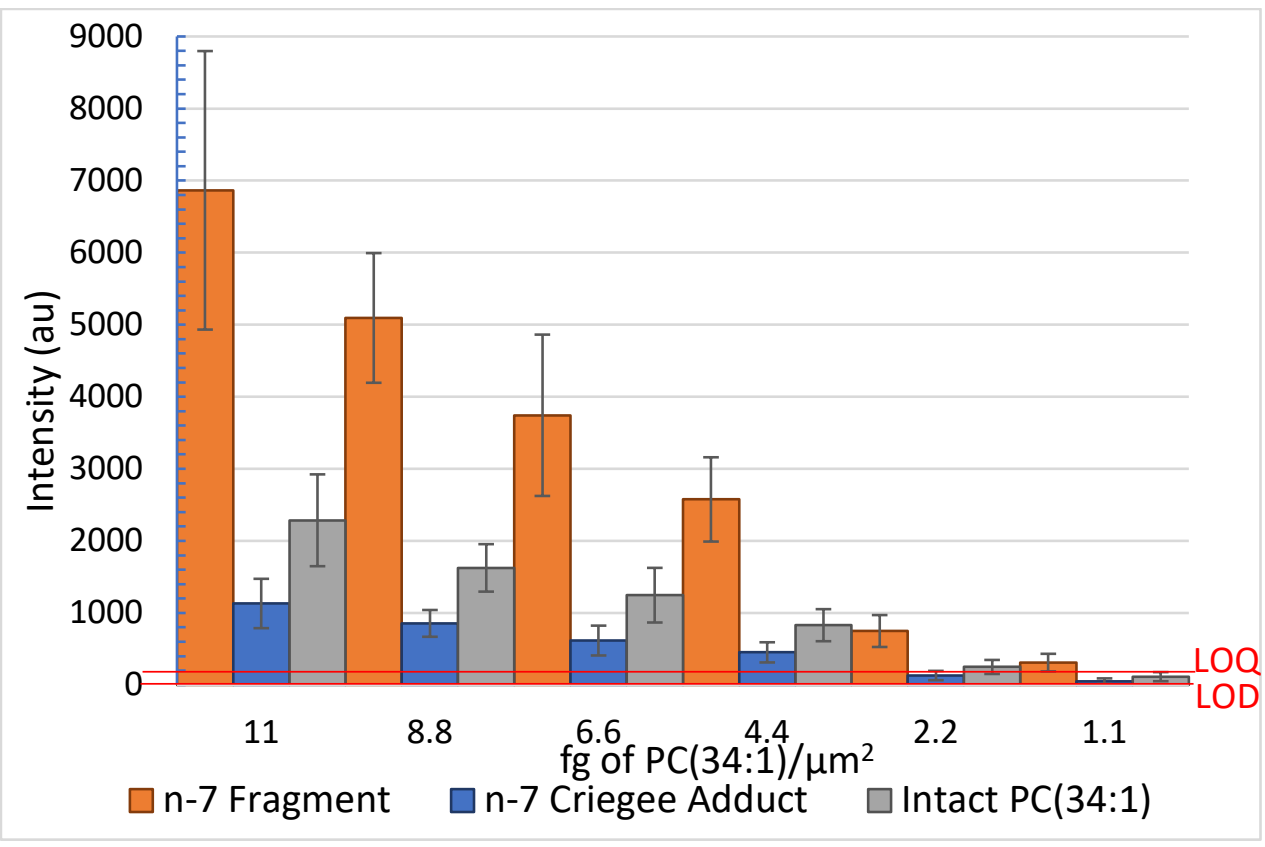

Figure 5.5. Sensitivity test to find the limit of detection. 1-10 layers of a PC 18:0/18:1n-9 standard were sprayed onto an ITO slide and measured by MALDI-OzID under the same conditions as the brain tissue sections. To examine the sensitivity, the abundance of the precursor and OzID product ions (the $n-9$ aldehyde and Criegee ions at $\mathrm{m} / \mathrm{z} 700.45$ and 716.48 , respectively) were monitored. Figure values are based on $\sim 850$ individual pixels acquired from each concentration. Characteristic OzID fragments and precursor signal for PC were observed in surface concentrations as low as $2.2 \mathrm{fg} / \mu^{2}$, only dropping below the limit of detection (LOD) at $1.1 \mathrm{fg} / \mu \mathrm{m}^{2}$, and being, on average, just above the limit of quantification (LOQ) at $2.2 \mathrm{fg} / \mu \mathrm{m}^{2}$. LOD and LOQ are defined as a S/N of 3 and 10, respectively. Noise level was determined to be 16 au, based on mMass single spectrum determination of 100 random pixels.

\section{DATA ANALYSIS}

WatersRawConverter was used to convert WRENS data using a bin size of $1 \mathrm{Da}$. The data was visualized using in-house built MATLAB scripts (version R2014a, The mathWorks, Natick, USA). Regions-of-interest (ROI) were manually selected to remove off-tissue regions when plotting images, with the tissue being TIC normalized. Ratio images were created as percentage of 100 , with the numerator being the sum of the aldehyde and Criegee fragment species of a single $\mathrm{db}$ isomer, and the denominator being the sum of all aldehyde and Criegee fragment species of all db isomers. Spectra were averaged in MassLynx v4.1 and loaded into mMass software[383] for offline recalibration and peak picking $(\mathrm{S} / \mathrm{N}=3)$. Calibrant peaks were most abundant OzID fragments (typically $n-7$ and $n-9$ ), along with phosphatidylcholine $(184.07 \mathrm{~m} / \mathrm{z})$ and ozonated precursor $([\mathrm{M}+48])$ 


\section{LIPID NOMENCLATURE}

Lipid structure nomenclature is based on the recommendations of Liebisch et al [384]. The site(s) of unsaturation are indicated by $n$-x, where the x-position is calculated from the methyl end. The underscore separator "_" is used when the snposition of the fatty acids is unknown, while the slash separator "/" is used if the $s n$-position of the fatty acid is known.

\section{RESULTS AND DiSCUSSION}

\section{MALDI-OZID IMAGING OF MONOUNSATURATED}

\section{PHOSPHATIDYLCHOLINES}

Db-isomer distributions of the abundant monounsaturated phosphatidylcholines, PC 34:1 and PC 36:1, were investigated in rat brain tissue to assess the sensitivity and speed of the high-pressure MSI-OzID setup (Figure 5.6). PC 34:1 and PC 36:1 have previously been studied with isomer-resolved MSI using OzID,[366, 385] UVPD,[161] and on-tissue PB reactions,[369] which provide benchmarks for this approach. The MALDI-OzID spectrum of [PC $34: 1+\mathrm{Na}]^{+}$ (Figure 5.6A) revealed product ions indicative of two db-positions, namely $n-7(\mathrm{~m} / \mathrm{z}$ 700.45 and 716.45) and $n-9(m / z 672.42$ and 688.42). Under optimized experiment conditions, OzID product ions were of comparable abundance to the mass-selected precursor ion, aiding isomer identification. Imaging of the intact precursor lipid distribution was performed in "TOF-only" mode on an adjacent tissue section from the same animal. The distribution of the $[\mathrm{PC} 34: 1+\mathrm{Na}]^{+}$ion at $<2 \mathrm{ppm}$ mass accuracy is shown in Figure 5.6B and represents the combined intensity distribution of signals arising from lipid isomers with this composition. The distributions of the individual PC 34:1n-7 and PC 34:1n-9 db-isomers were extracted from the OzID data and were individually compared against the total abundance of product ions from all isomers to generate fractional distribution images (FDI), as shown in Figure 5.6C. Comparing the sum composition distribution with the isomer-resolved image reveals that although PC 34:1 shows higher abundance in the gray matter of the brain (Figure 5.6B), an underlying variation in the relative population of $\mathrm{PC}$ 34:1n-7 and PC 34:1n-9 is present (Figure 5.6C). Comparison between the isomerresolved-MSI and H\&E-stained tissue revealed the $n-7$ isomer is proportionately more abundant in gray matter and the cerebellum and less abundant in white matter and the cortex, consistent with previous isomer-resolved MSI studies.[366, 368- 
370] Compared to prior isomer resolved MSI implementations, the acquisition time for the data reported here $(\sim 4 \mathrm{~h})$ is significantly shorter than would have been required to acquire equivalent data using earlier OzID-MSI[366] ( 200 h) or UVPD-MSI[367] $(\sim 36 \mathrm{~h})$ implementations, while simultaneously improving sensitivity for the diagnostic fragment ions. 
(A)

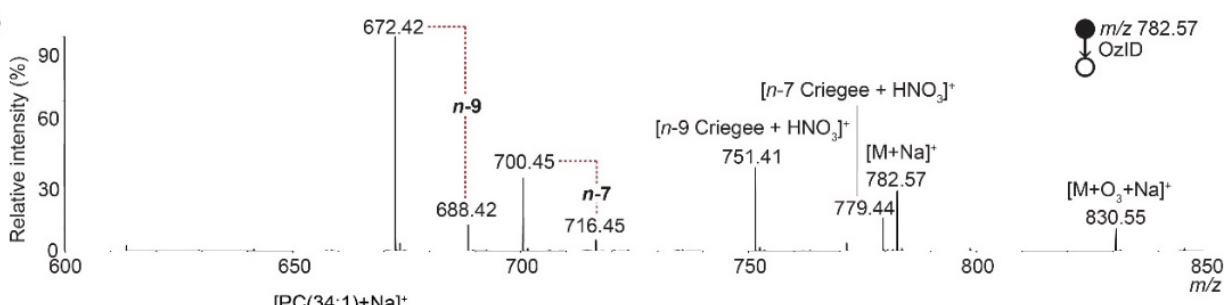

(B)

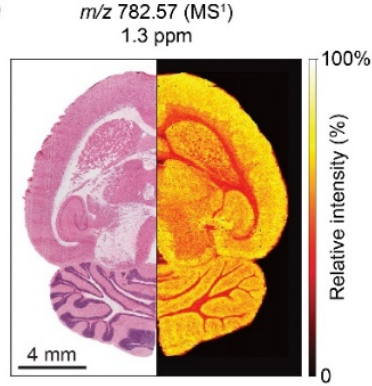

(D)

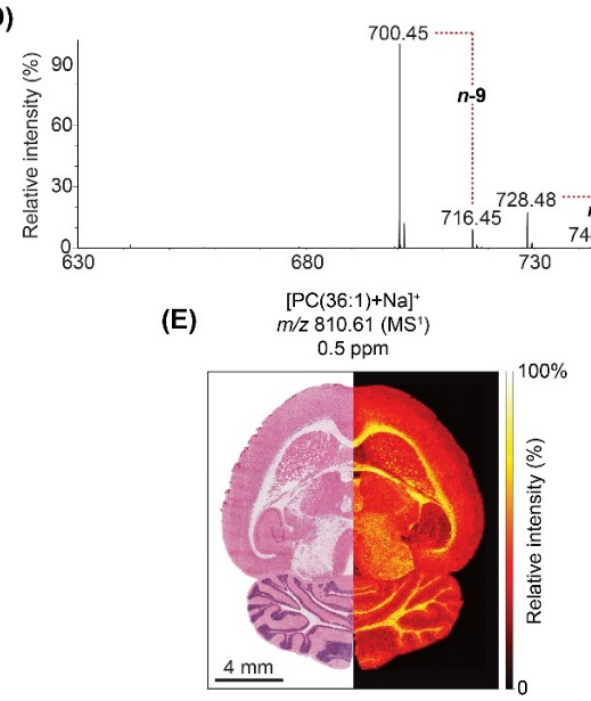

(C)

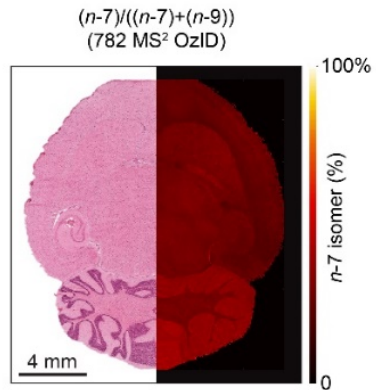

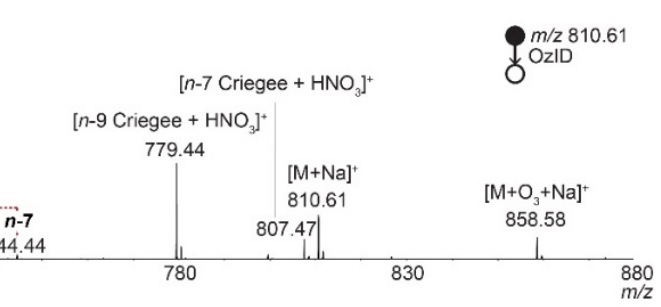

(F)

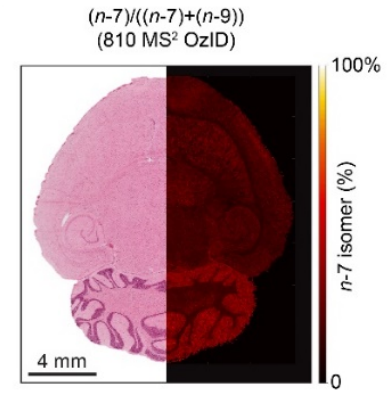

Figure 5.6 MALDI-OzID of monounsaturated (A-C) [PC 34:1+Na $]^{+}$and (D-F) [PC 36:1+Na $]^{+}$, showing different isomer distributions in rat brain. MALDI-OzID spectrum of (A) $[\mathrm{PC} 34: 1+\mathrm{Na}]^{+}$and (D) $[\mathrm{PC} 36: 1+\mathrm{Na}]^{+}$where labeled product ions indicate the presence of $n-7$ and $n-9 \mathrm{db}$ isomers. Distribution of precursor ions (B) [PC $34: 1+\mathrm{Na}]^{+}$at $\mathrm{m} / z 782.57$ and (E) [PC 36:1+Na] ${ }^{+}$at $\mathrm{m} / z 810.61$ extracted from TOF-only scans. Fractional distribution images of (C) PC 34:1n-7 and (F) PC 36:1n-7 obtained from MALDI-OzID, showing relative changes in $n-7$ isomers throughout the rat brain. In panels B-C and E-F, H\&E staining of the same tissue after the MSI experiments is shown on the left. Note that MALDI-OzID and MALDI-TOF images are acquired from different brain tissue sections from the same animal. Explanation of addition ions present in (A) is provided as [366][366]Figure 5.7. 


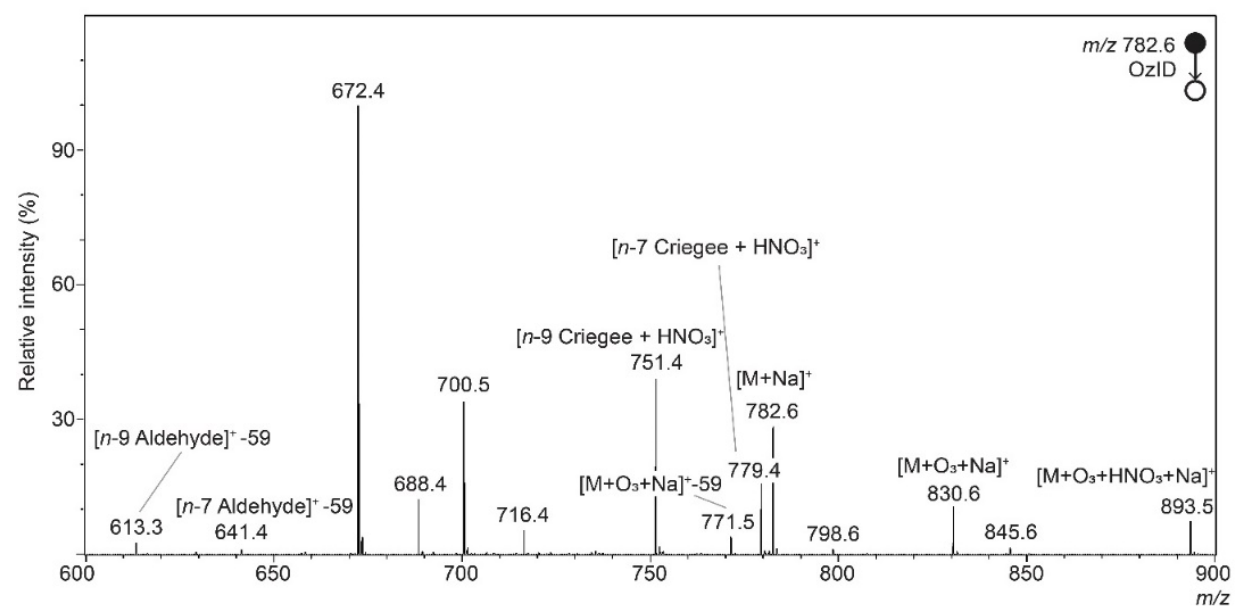

Figure 5.7 Explanation of additional ions observed in figure 1A. Aside from the regular $n-7$ and $n-9$ OzID fragments, other ozone products were present as well. The $m / z 830.6$ ion was assigned as the $\left[\mathrm{M}^{2} \mathrm{O}_{3}+\mathrm{Na}\right]^{+}$, previously reported by Paine $e t$ al. ${ }^{1}$ who observed the formation of this stable secondary ozonide during gas-phase ozonolysis of ionized lipids. Nitric acid (a minor byproduct of ozone generation) adducts were observed for Criegee ions ( $\left[n-7 \text { Criegee }+\mathrm{HNO}_{3}\right]^{+}, m / z$ 779.4; $\left[n-9 \text { Criegee }+\mathrm{HNO}_{3}\right]^{+}, m / z$ 751.4) and secondary ozonide ions, $\left[\mathrm{M}+\mathrm{O}_{3}+\mathrm{HNO}_{3}+\mathrm{Na}\right]^{+}$at $m / z$ 893.5. Furthermore, product ions associated with the characteristic neutral loss of trimethylamine (NL -59 Da) from the PC headgroup were also observed from the OzID ions. These ions were $\mathrm{m} / \mathrm{z}$ $771.5\left(\left[\mathrm{M}+\mathrm{O}_{3}+\mathrm{Na}\right]^{+}-59\right), m / z 641.4(-59 \mathrm{Da}$ from the $n-7$ aldehyde ion) and $m / z 613.4(-59 \mathrm{Da}$ from the $n-9$ aldehyde ion).

A representative MALDI-OzID spectrum of mass-selected [PC 36:1+Na] ${ }^{+}$ ions is shown in Figure 5.6D and reveals the presence of both $n-7(\mathrm{~m} / z 728.48$ and $744.44)$ and $n-9(m / z 700.45$ and 716.46) db isomers, in agreement with previous studies.[366, 380] The precursor ion distribution of $m / z 810.61$ showed that PC 36:1 was concentrated throughout the white matter (Figure 5.6E), in contrast to the correlation between PC 34:1 and gray matter. As was also observed in previous studies, $[366,386]$ the FDI (Figure 5.6F) revealed that the $n-7$ isomer was more prevalent in the gray matter and the cerebellum, while the $n-9$ was more abundant in the white matter and the cortex.

MALDI-OZID OF POLYUNSATURATED PC SPECIES 

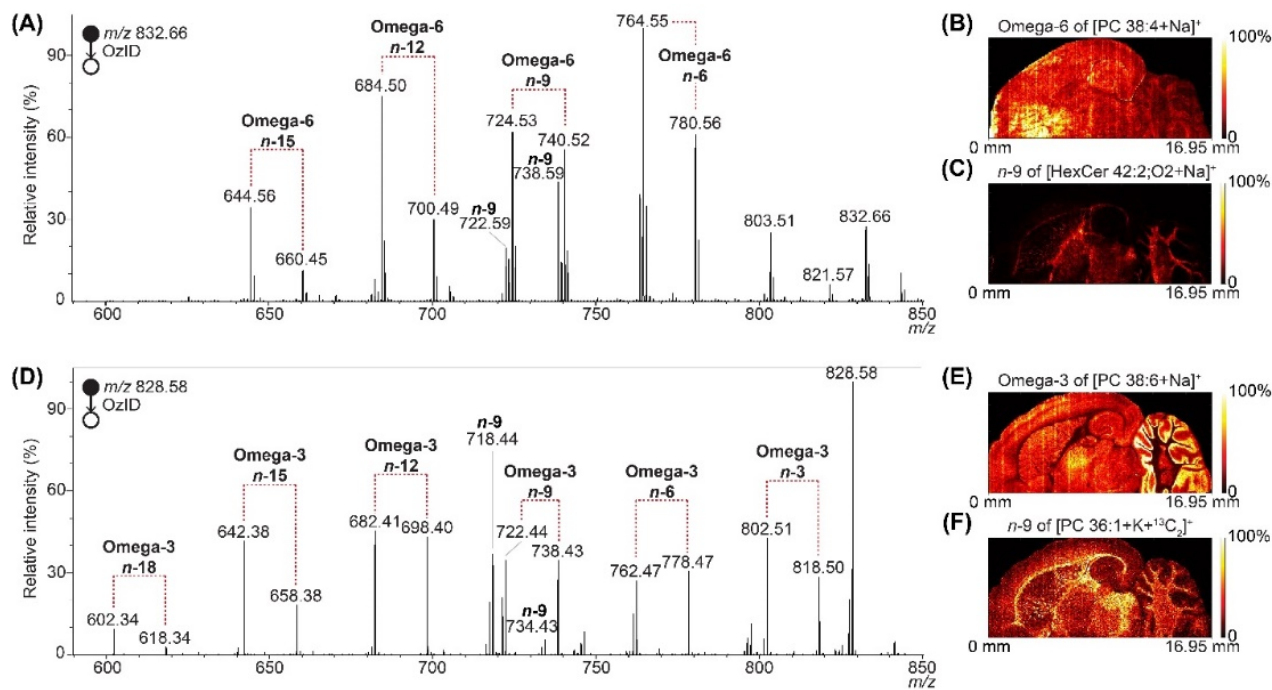

Figure 5.8 OzID of polyunsaturated PC lipids showing the distribution of the polyunsaturated phospholipid and an interfering lipid throughout the rat brain, showing different distributions in white/gray matter. (A) MALDIOzID spectrum of $[\mathrm{PC}(38: 4)+\mathrm{Na}]^{+}$, revealing the presence of an omega- 6 fatty acid. In addition, $n-9$ OzID fragments from an interference are visible in the spectrum, labelled as $n-9$. (B) Distribution images of the omega3 (top) and the $n-9$ OzID fragments (bottom). (C) MALDI-OzID spectrum of [PC(38:6)+Na] $]^{+}$, revealing the presence of an omega-3 fatty acid. In addition, $n-9$ OzID fragments from an interference are visible in the spectrum, labelled as $n-9$. (D) Distribution images of the omega-3 (top) and the $n-9$ OzID fragments (bottom).

The increased sensitivity of the MALDI-MSI-OzID implementation is particularly important for polyunsaturated lipids because the diagnostic double bond positional information is spread across multiple pairs of OzID fragments. OzID of mass-selected [PC $38: 4+\mathrm{Na}]^{+}$and [PC $\left.38: 6+\mathrm{Na}\right]^{+}$produced spectra with sufficient $\mathrm{S} / \mathrm{N}$ to allow spatial distributions to be extracted for each double bond position (see Figure 5.8 and Figure 5.9). The MALDI-OzID spectrum of [PC $38: 4+\mathrm{Na}]^{+}$revealed four double bonds at the $n-6,9,12$, and 15 positions (Figure 5.8A), which enables assignment of the lipid as PC 18:0_20:4n-6. Isobars around the $n-9$ neutral loss indicated the presence of an isobaric monounsaturated lipid, assigned as hexosylceramide HexCer 42:2;O2 by accurate mass. OzID imaging of $[\mathrm{HexCer} 42: 2 ; \mathrm{O} 2+\mathrm{Na}]^{+}$revealed that the distribution of this sphingolipid was distinct from PC 38:4 (Figure 5.8B and C). While PC 38:4 was widely distributed throughout the gray matter (Figure 5.8B), HexCer 42:2; O2 was highly localized to the white matter/myelin, which is known to be rich in glycosphingolipids (Figure $5.8 \mathrm{C})$. 
(A)

[PC 38:4+Na]+
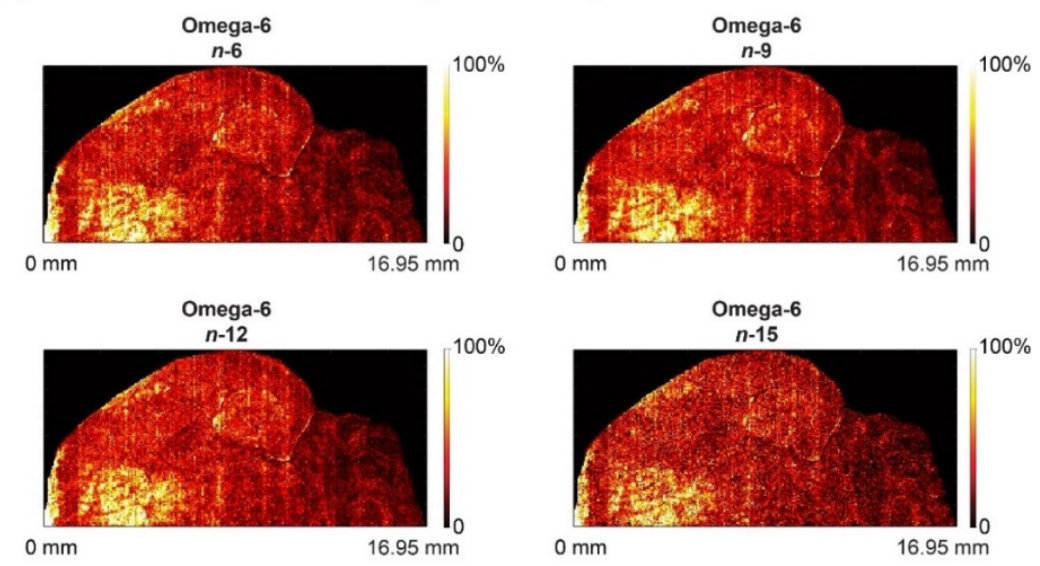

(B)

$[\mathrm{PC} 38: 6+\mathrm{Na}]^{+}$
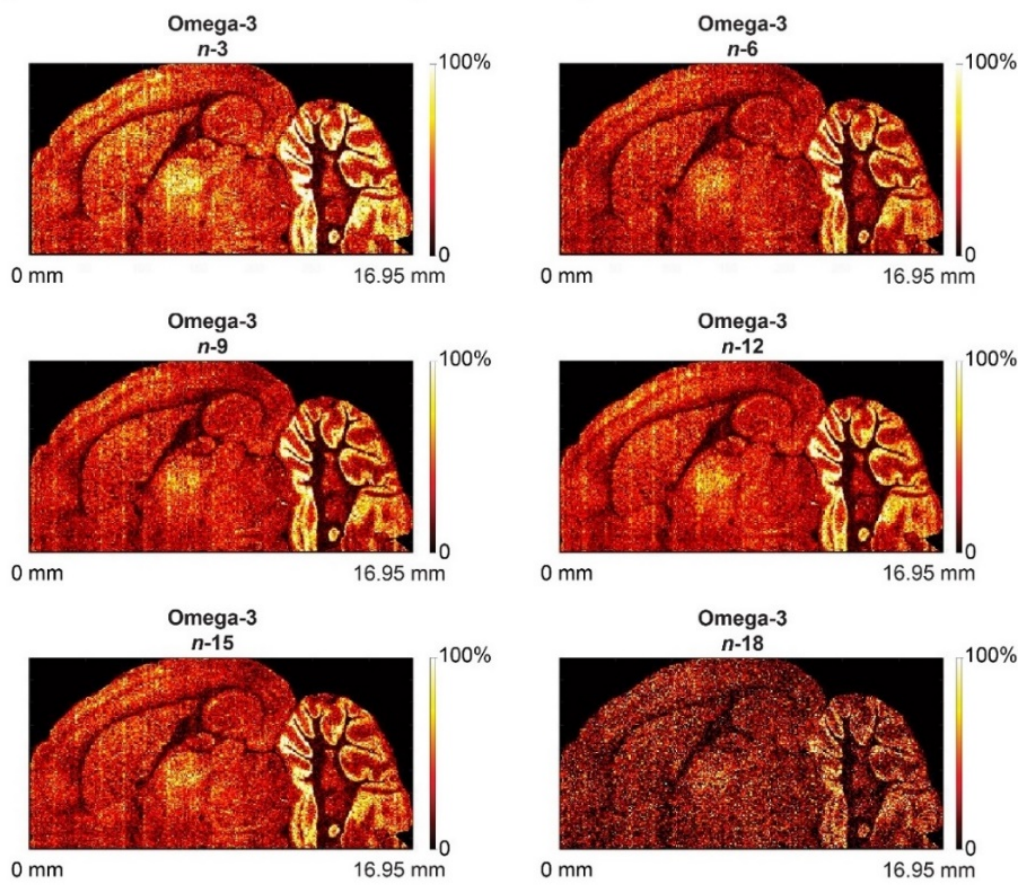

Figure 5.9 Spatial distribution of each pair of OzID ions corresponding to each carbon-carbon double bond in the polyunsaturated lipids PC 38:4 and PC 38:6. (A) Spatial distribution images of the ions arising from each of the four $\mathrm{db}$ positions of [PC $38: 4+\mathrm{Na}]^{+}$showing the same distribution for all db within the omega- 6 fatty acyl chain. (B) Spatial distribution images of the six db positions of [PC 38:6+Na] $]^{+}$showing the same distribution for all $\mathrm{db}$ within the omega-3 unsaturation. For these images, $99^{\text {th }}$ quantile hotspot removal was performed on the nonnormalized images. 
For [PC 38:6+Na] $]^{+}$(Figure 5.8D), the MALDI-OzID spectra showed six pairs of product ions corresponding to double bonds at the $n-3,6,9,12,15$, and 18 positions, assigning the lipid as PC 16:0_22:6n-3, which has been previously reported in rat brain[366] and is shown here to be primarily present in the granular cell layer of the cerebellum (Figure 5.8E). As with PC 38:4, isobars around the $n-9$ neutral loss revealed the presence of an additional monounsaturated lipid, in this case the $\left[\mathrm{PC} 36: 1+\mathrm{K}+{ }^{13} \mathrm{C}_{2}\right]^{+}$isotopologue in the gray matter (Figure 5.8F). Interestingly, in these spectra we observed an "inverted" isotopic distribution, created from the loss of either one or both ${ }^{13} \mathrm{C}$ in the OzID neutral loss fragment. This could potentially be used as a diagnostic pattern for identifying isobaric and co-isolated ${ }^{13} \mathrm{C}$-containing lipids (Figure 5.10). 


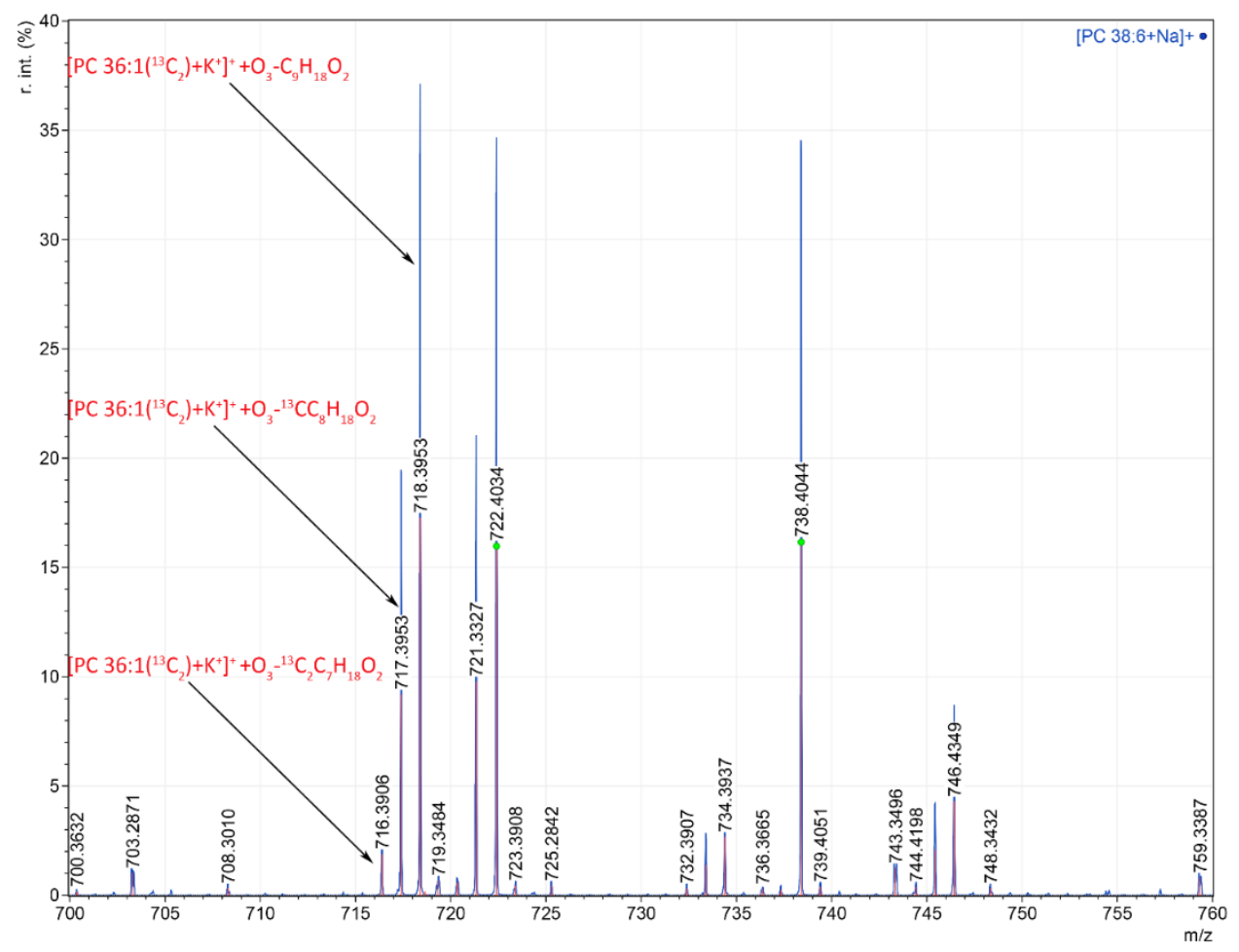

Figure 5.10 Magnified view of the MALDI-OzID spectrum from mass selection of $\mathrm{m} / \mathrm{z} 828$ that co-isolates [PC $38: 6+\mathrm{Na}]^{+}$and the M+2 isotopologue of [PC $\left.36: 1+\mathrm{K}\right]^{+}$. Peaks corresponding to OzID of the $n-9$ double bond of PC 36:1 are observed at $m / z 718.44$ and 734.43 and exhibit an inverse isotope pattern arising from OzID neutral losses that carry 0,1 , or $2{ }^{13} \mathrm{C}$ isotopes, with the remaining charged fragment containing $2{ }^{13} \mathrm{C}$ (at 718.3953 ), $1{ }^{13} \mathrm{C}$ (717.3953), and no ${ }^{13} \mathrm{C}(\mathrm{m} / \mathrm{z} 716.3906)$.

\section{MALDI-CID-OzID² OF MONOUNSATURATED PC SPECIES}

CID/OzID has been used to identify sn-positional isomers of PC lipid species via an $\mathrm{MS}^{3}$ sequence. As only $\mathrm{MS}^{2}$-level activation could be performed with the SYNAPT, the CID products could not be mass-selected prior to ozonolysis. Instead, to generate CID/OzID product ions, a combined collision- and ozoneinduced dissociation (COzID) strategy was employed.[387] Mass selected $[\mathrm{M}+\mathrm{Na}]^{+}$ions were activated in the trap region (i.e. prior to ozonolysis) with a 40 $\mathrm{V}$ collision energy, forming $[\mathrm{M}-183+\mathrm{Na}]^{+}$head group loss fragment ions without significantly depleting the $[\mathrm{M}+\mathrm{Na}]^{+}$precursor. Subsequent reaction of these ions with ozone produced both CID/OzID and OzID product ions, providing $\mathrm{db}$ - and $s n$ positional isomer information in the same spectrum. This experiment sequence was performed for [PC 34:1+Na] $]^{+}$(Figure 5.11A), revealing product ions indicative of 
both $n-7(\mathrm{~m} / \mathrm{z} 700.47$ and 716.47$)$ and $n-9(\mathrm{~m} / \mathrm{z} 672.47$ and 688.47) db positions in addition to CID/OzID fragments diagnostic for both 16:0 and 18:1 acyl chains at the $s n-1$ position $(m / z 379.25 / 395.25$ and $405.25 / 421.26$, respectively; see Figure 5.12A). In accordance with previous investigations, the fractional distribution showed the canonical PC 16:0/18:1 is elevated within the white matter, while the non-canonical PC 18:1/16:0 isomer is proportionately elevated in the gray matter (Figure 5.11B).[366] 


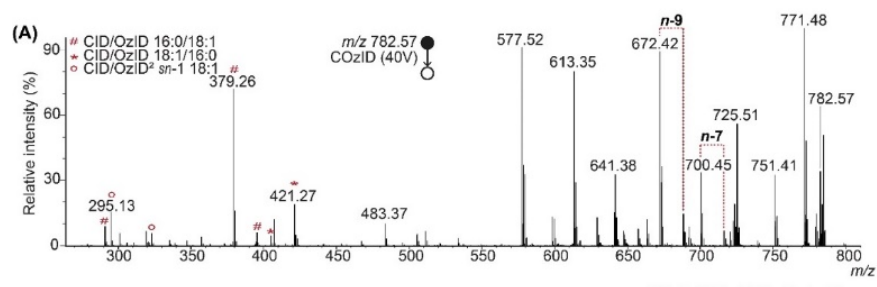

(B)
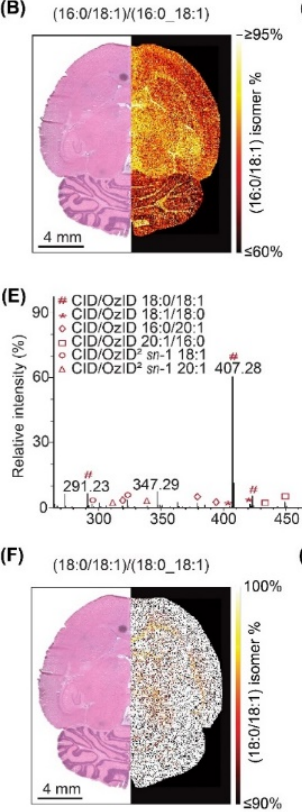

(I) (CID-OzID ${ }^{2}(n-7) /((n-7)+(n-9))$

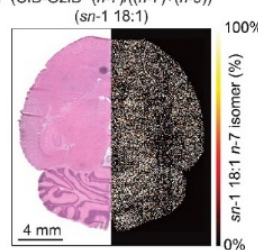

(G)
(C) $\quad(n-7) /((n-7)+(n-9))$

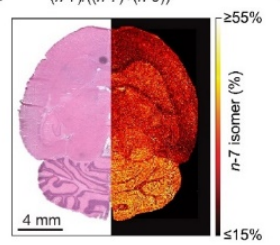

(D) $\mathrm{CID}-\mathrm{OzID}{ }^{2}(n-7) /((n-7)+(n-9))$

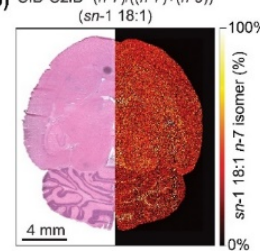

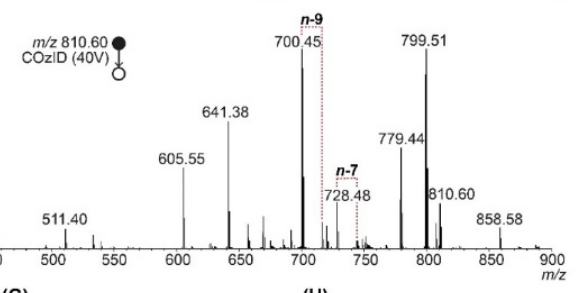

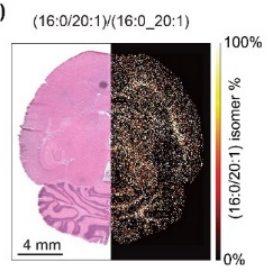

(H)

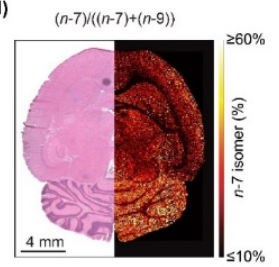

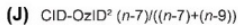

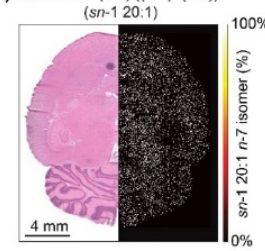

Figure 5.11 MALDI-COzID of (A-D) $[\mathrm{PC} 34: 1+\mathrm{Na}]^{+}$and (E-J) $[\mathrm{PC} 36: 1+\mathrm{Na}]^{+}$in rat brain obtained by preactivating the ions with $\mathrm{CID}(40 \mathrm{~V})$ in the trap region prior to ozonolysis in the ion-mobility cell. (A) COzID mass spectrum of [PC 34:1+Na] $]^{+}$showing diagnostic product ion pairs indicating the presence of db-positional (PC 34:1n-7 and PC 34:1n-9) and sn-positional (PC 16:0/18:1 and PC 18:1/16:0) isomers. Additional product ions observed in the spectrum are consistent with $\mathrm{CID} / \mathrm{OzID}{ }^{2}$ processes, which are regiospecific for the $n-7$ and $n-9 \mathrm{db}-$ positions of the $s n-1$ 18:1. (B-D) FDIs of the different isomers present, showing the intensity of signals specific for (B) PC 16:0/18:1, (C) PC 34:1n-7, and (D) [PC 18:1(n-7)/16:0+Na] isomers. (E) COzID spectrum of [PC $36: 1+\mathrm{Na}]^{+}$showing diagnostic product ion pairs indicating the presence of db-positional (PC $36: 1 n-7$ and $\mathrm{PC}$ 36:1n-9) and sn-positional (PC 18:0/18:1 and PC 18:1/18:0, PC 16:0/20:1 and PC 20:1/16:0) isomers. Additional product ions observed in the spectrum are consistent with $\mathrm{CID} / \mathrm{OzID}^{2}$ processes, which are regiospecific for the $n$ 7 and $n-9$ db-positions of the $s n-118: 1$ and $s n-1$ 20:1. (F-J) FDIs of the different isomers present, showing the intensity of (F) the PC 18:0/18:1, (G) PC 16:0/20:1, (H) PC 36:1(n-7) db-position, (I) [PC 18:1(n-7)/18:0+Na] ${ }^{+}$, and (J) [PC 20:1(n-7)/16:0+Na] $]^{+}$isomers. For the FDIs, an H\&E staining of a consecutive section is shown on the left. 
(A)

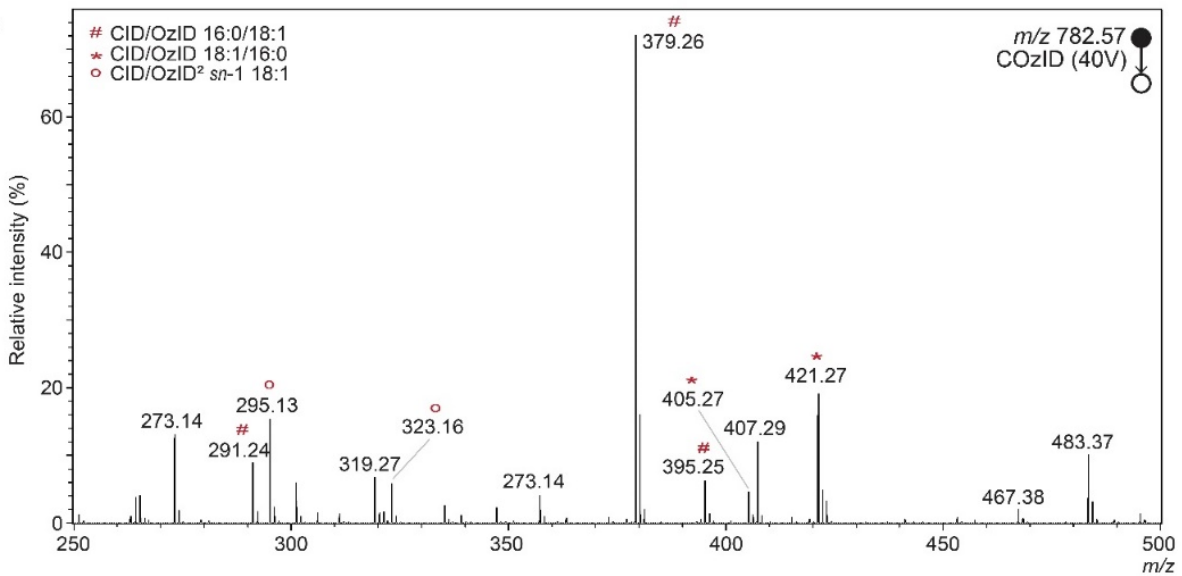

(B)

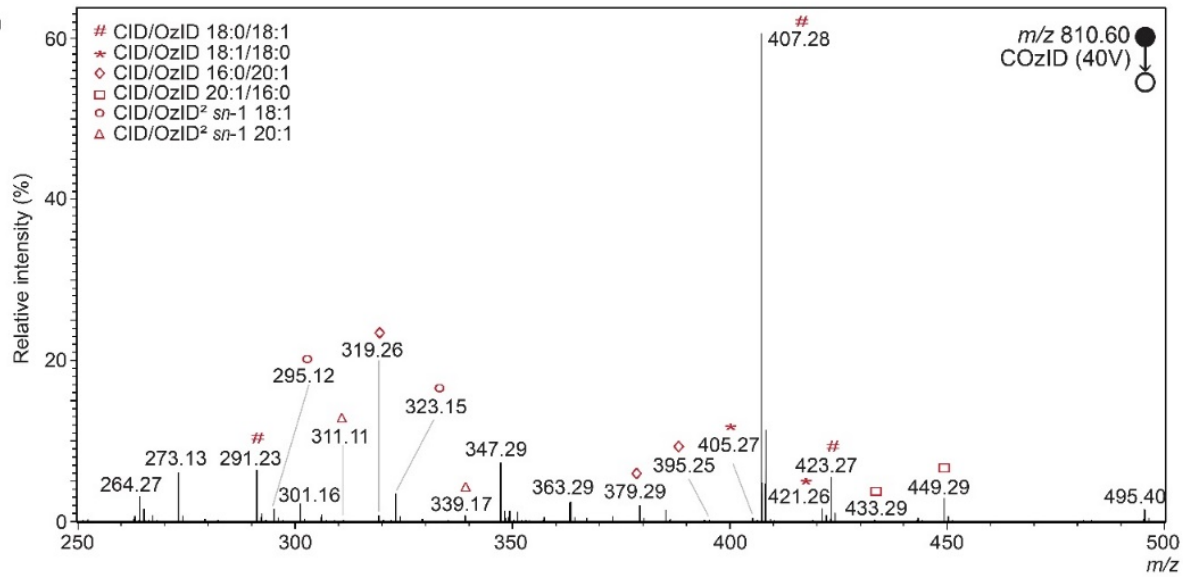

Figure 5.12 Enlargement of $m / z$ 250-500 of the COzID spectra of (A) [PC 34:1+Na $]^{+}$and (B) $[\mathrm{PC} 36: 1+\mathrm{Na}]^{+}$.

The observation of both $\mathrm{db}$ - and $s n$-specific product ions in a single spectrum (Figure 5.11A) supports the presence of at least three, and likely four, PC 34:1 isomers (i.e., PC 16:0/18:1n-7, PC 16:0/18:1n-9, PC 18:1n-7/16:0 and PC 18:1n-9/16:0). Additional information can be gleaned from low mass ions (i.e., $m / z$ 250-350) in the same spectra the presence of which, indicates subsequent OzID reactions on CID/OzID fragment ions (i.e., CID/OzID ${ }^{2}$ product ions). These ions have been characterized previously by Pham et al. using explicit $\mathrm{MS}^{4}$ activation on an ion-trap mass spectrometer[165]. Importantly, these product ions allow unambiguous assignment of db-locations to $s n-1$ acyl chains based on the fragmentation sequence outlined in Figure 5.13. For example, in the case of [PC 
$34: 1+\mathrm{Na}]^{+}$(Figure 5.11A), the CID/OzID ${ }^{2}$ ions observed at $\mathrm{m} / \mathrm{z} 295.12$ and 323.15 reveal the presence of lipid isomers carrying both $n-7$ and $n-9 \mathrm{db}$ locations at the $s n-1$ position, namely the non-canonical PC 18:1n-9/16:0 and PC 18:1n-7/16:0 lipids. Pham et al. also identified an alternative $\mathrm{MS}^{4}$ sequence, which uniquely assigns db isomers of the $s n-2$ substituted acyl chains. Unfortunately, these low $m / z$ product ions were not detected under the COzID conditions used, however $s n-2$ unsaturation can be inferred from the comparison between OzID and COzID spectra. In rat brain, $\mathrm{CID} / \mathrm{OzID}^{2}$ product ions showed unique distributions of [PC $18: 1 n-7 / 16: 0+\mathrm{Na}]^{+}$and $[\mathrm{PC} 18: 1 n-9 / 16: 0+\mathrm{Na}]^{+}$. Although the overall signal from PC 34:1n-7 was relatively more abundant in the gray matter of the cerebellum (Figure 5.11C), the non-canonical PC 18:1n-7/16:0 isomer appeared more abundant in the white matter of the brain (Figure 5.11D). Despite our inability to directly visualize $s n-2$ related $\mathrm{db}$ fragments in this experiment, a comparison between Figure 5.11C and D indicates that the location of double bonds in fatty acids is closely correlated with the $s n$-position of the fatty acid. 


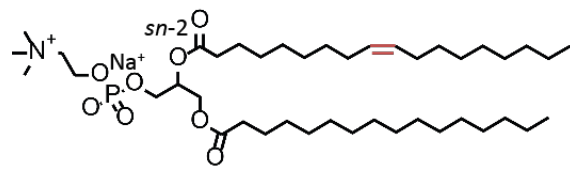

Exact Mass: 782.5670

16:0/18:1

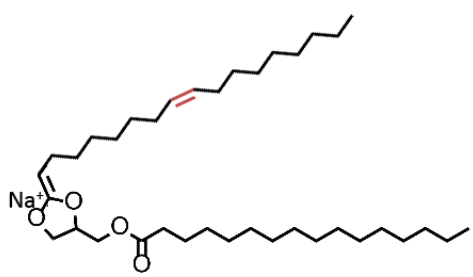

Exact Mass: 599.5010

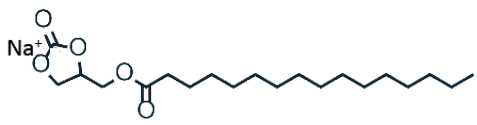

Exact Mass: 379.2455

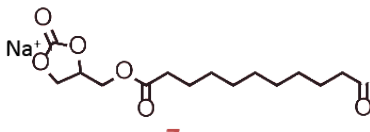

$n-7$

Exact Mass: 323.1465

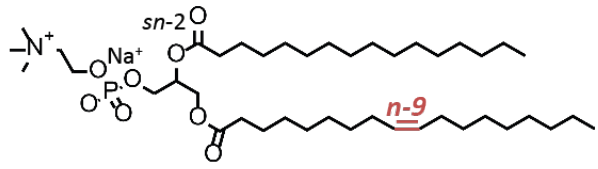

Exact Mass: 782.5670

18:1/16:0

(5ms CID)

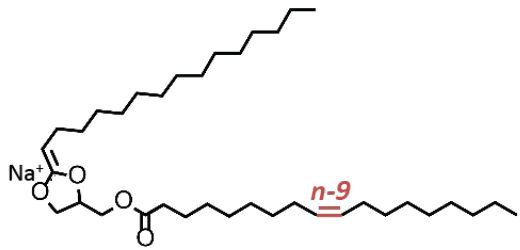

Exact Mass: 599.5010

$\mathrm{O}_{3}$
$(10 \mathrm{~ms})$
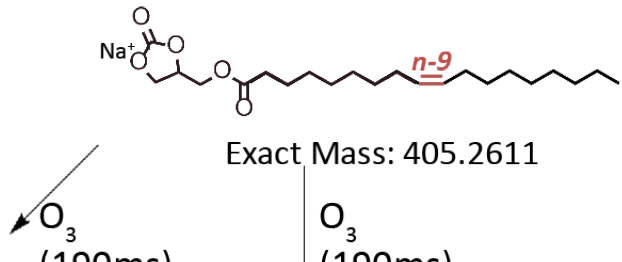

Exact Mass: 405.2611

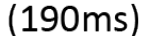

if $n-7$ instead

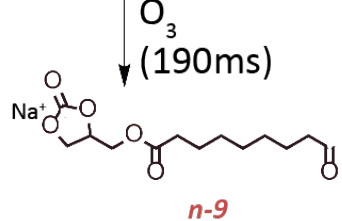

Exact Mass: 295.1152

Figure 5.13 Proposed CID/OzID ${ }^{2}$ reaction scheme for [PC 16:0_18:1+Na] $]^{+}$, where CID/OzID product ions undergo a further reaction with ozone, leading to diagnostic fragments observed at $\mathrm{m} / \mathrm{z} 295.12$ and 323.15 , indicative of both the 18:1(n-9)/16:0 and 18:1(n-7)/16:0 isomers, respectively.

The equivalent analysis for $[\mathrm{PC} 36: 1+\mathrm{Na}]^{+}$revealed the presence of both $n-7$ and $n-9$ isomers for each PC 18:0/18:1, PC 18:1/18:0, PC 16:0/20:1, and PC 20:1/16:0 (Figure 5.11E; magnification of $m / \mathrm{z} 250-500$ is provided in the Figure 5.12B). In line with previous investigations of [PC $36: 1+\mathrm{Na}]^{+}$, these data reveal that PC 18:0/18:1 accounts for $>90 \%$ of all PC 18:0_18:1 sn-isomers (Figure 5.11F)[366] based on relative intensity, and the relative fractions of $\mathrm{PC} 18: 0 / 18: 1$ and PC 18:1/18:0 do not change significantly throughout the brain. By comparison, PC 16:0/20:1 relative to the combined PC 16:0_20:1 signal is confined almost completely to the white matter (Figure 5.11G). The FDI of the db-locational 
isomers revealed PC $36: 1 n-7$ to be relatively more abundant in the gray matter and cerebellum compared to the cortex (Figure 5.11H). Although the FDIs based on CID/OzID 2 product ions had lower signal-to-noise for PC 36:1, PC 18:1n-7/18:0 and PC 20:1n-7/16:0 isomers could still be observed, however $n-9$ isomer species appeared entirely absent (Figure 5.11I-J). This indicates that the $n-9 \mathrm{db}$ is exclusively associated with the 18:1 or 20:1 chain substituted at the $s n-2$ position or was present below the detection limit of our analysis.

\section{CONCLUSION}

The data presented herein demonstrate that OzID imaging data can be obtained at 5 pixels/s due to the higher sensitivity and faster reaction speed with this method. This allows for either increasing the analytical detail by using higher spatial resolution or acquire bigger tissue areas or increasing the throughput. In addition, isobaric interferences can be resolved with this method, without the need for high mass resolution. Aside from regular OzID, also CID/OzID experiments can be performed, which showed to even further oxidize to form CID-OzID ${ }^{2}$ fragments specific for the db-position at the $s n-1$ fatty acid. To our knowledge, this is the first time both pieces of isomeric information, including the specificity of the MUFAs on the sn-1 position, were acquired in a single imaging experiment. Hence, our method overcomes several limitations of lipid structure characterization in mass spectrometry imaging, which will help to gain a better understanding of localized lipidomic processes on complex tissues in the future. 


\section{Chapter 6 NegAtive Mode}

IMAGING OF PHOSPHOLIPIDS USING OZONE-INDUCED DISSOCIATION

Andrew P. Bowman ${ }^{1 \#}$, Britt S.R. Claes ${ }^{1 \#}$, Berwyck L.J. Poad ${ }^{2}$, Ron M.A. Heeren ${ }^{1}$, Stephen J. Blanksby ${ }^{2}$ and Shane R. Ellis ${ }^{1,3 *}$

${ }^{1}$ The Maastricht MultiModal Molecular Imaging (M4I) Institute, Division of Imaging Mass Spectrometry (IMS), Maastricht University, 6229 ER Maastricht, The Netherlands

${ }^{2}$ Central Analytical Research Facility, Queensland University of Technology, Brisbane, Queensland 4001, Australia

${ }^{3}$ Mass Spectrometry Imaging Laboratory, School of Chemistry and Molecular Bioscience, University of Wollongong, Wollongong, New South Wales 2522, Australia

\# These authors have equally contributed 


\section{INTRODUCTION}

The identification of biomarkers in assessing both the health of tissue and the progress of disease is one of the key challenges facing clinicians. Within that context, lipidomics is a highly important field of studying, owing to the fact that lipids are the main components of cell structure[41] and are utilized as signaling molecules[327] for diverse outcomes stretching from apoptosis[329] to metabolism, whether that metabolism is healthy[388] or cancerous[22]. It is well known that the utilization of lipids change based on their chemical structure, with relatively minor changes indicating sweeping physiological differences.[333, 334] Add to this the evidence revealing the change of lipid synthesis dependent upon tissue[389], cancer metabolism,[22, 385], or metabolic disorders such as diabetes,[344] and the fact that identifying specific lipid isomers becomes obvious.

One of the main issues plaguing mass spectrometry in the identification of lipids is the lack of sufficient structural characterization in conventional methodologies.[390-392] Poor specificity comes from several factors, chiefly in the combination of isobaric (similar mass) and isomeric (structurally distinct) species. These result in the generation of confounded images that are the overlap of multiple species rather than a singular lipid. This is compounded within mass spectrometry imaging (MSI), where the very nature of most ionization techniques prohibits the inclusion of a separatory step that could distinguish between isomers. To improve the resolution of lipids, different technologies have been implemented. Condensed-phase chromatographic separations have been utilized to improve isomeric and isobaric resolution but are typically incompatible with MSItimescales. High resolution mass spectrometers can be used to improve isobar resolution, with the most powerful Fourier-transform-based instruments providing sub-parts-per-million mass accuracy and resolving power. However, even in such cases where sufficiently narrow mass windows can be chosen to exclude isobaric compounds, isomers cannot be separated by mass alone. Ion mobility experiments can separate some isomers, though the utility for all lipid isomers has never been demonstrated.

As such, MSI experiments to identify the specific localization of resolved lipid isomers has been pushed forward by a number of different MS/MS methodologies. The most common form of MS/MS has been collision-induced dissociation (CID), being available on most commercial instruments. CID 
experiments can identify the sum composition of most phospholipids by identifying the headgroup of the phospholipid and by extension the total carbon number in the acyl chains. In negative mode, CID can also identify individual acyl chains, which ionize well as fragments from the phospholipid. However, CID fails to provide any information relating to the position of double bonds, nor, in general, does it identify the stereonumbering (attachment on the glycerol backbone) of the fatty acyl chains. Multiple techniques have been developed that can aid in the identification of double bond position: the ability to perform isomer-resolved imaging of phospholipids has been demonstrated using ultraviolet photodissociation (UVPD)[367], ion-ion reactions,[162] ozone-induced dissociation (OzID),[366] electron impact for excitation of ions from organics (EIEIO),[393, 394] epoxidation,[163] and the Paterno-Buchi (PB) reaction.[164] However, many of these techniques have either only been demonstrated on phosphatidylcholine in the positive mode or have not been demonstrated in conjunction with mass spectrometry imaging.

OzID in MSI has, to date, only been performed in the positive mode and only with phosphatidylcholine. This has been due to the slow rate of reaction impeding sensitivity in the highly abundant, metal-cationized PCs. While phosphatidylethanolamine is the most abundant lipid species in animal biology (making up $\sim 54 \%$ of the phospholipids in brain tissue)[395], the lack of metalassistance to promote the ion/molecule interaction of OzID has impeded its use in negative mode, let alone within the other, less abundant phospholipid classes. Further, without the metal cation the two-stage process of CID/OzID is unavailable, making identification of specific stereonumbering difficult. Despite these limitations, our recent implementation of OzID in a Synapt G2Si system, which boosted the reaction rate in comparison to older work by more than 50 -fold, has enabled the use of OzID in the negative mode.

Herein, we demonstrate the utility of OzID to identify double bond location in multiple phospholipid classes, including phosphatidylethanolamine (PE), phosphatidylinositol (PI), phosphatidylserine (PS), phosphatidylglycerol (PG), and phosphatidic acid (PA). We combine CID and OzID into a single Collision-Ozone Induced Dissociation (COzID), enabling simultaneous identification of acyl chain and double-bond $(\mathrm{db})$ isomerization. We present these identifications using both healthy brain tissue, as well as medullablastoma-bearing brain tissue, and lowdensity-lipoprotein negative kidney tissue. 


\section{Methods}

\section{MATERIALS}

2,5-Dihydroxybenzoic acid (DHB, HPLC-grade, $\geq 99 \%$ purity) and chloroform ( $\geq 99 \%$ ) were purchased from Sigma Aldrich (Zwijndrecht, The Netherlands) and used without further purification. Methanol (ULC-MS grade), water (LC-MS grade), ethanol (LC-MS grade), and xylene (AR grade) were purchased from Biosolve (Valkenswaard, The Netherlands). Hematoxylin and Entellan ${ }^{\circledR}$ were purchased from Merck (Darmstadt, Germany) and eosin Y from J.T. Baker (Center Valley, USA). Indium tin oxide (ITO)-coated glass slides were purchased from Delta Technologies (Loveland, USA). Lipid standards (PE 16:018:1, PE 18:0-18:1, PS 16:0-18:1, PS 18:0-18:1) were purchased from Avanti Polar Lipids (Alabaster, USA).

\section{BIOLOGICAL SAMPLES}

Rats, housed and cared for at the Central Animal Facility of Maastricht University according to local standards, were provided ad libitum access to water and regular chow. Healthy rat brain (HRB) was obtained in accordance with protocols approved by the Animal Care and Use Committee (DEC number 2014120). MedullaBlastoma-bearing Mouse Brains (BMB) from Transgenic ND2:SmoA1-GFP mice were housed and cared for at Emory University and used in accordance with protocols approved by the Emory Institutional Animal Care and Use Committee. One mouse kidney (MK) was collected from an LDL R-/- mice from a protocol approved by the Animal Care and Use Committee (DEC number 2014-069) at Maastricht University. Mice were provided ad libitum access water and regular chow. Animals were housed and cared for at the Central Animal Facility of Maastricht University according to local standards. Horizontal sections of all tissues measuring $10 \mu \mathrm{m}$ thick were prepared using a cryo-microtome (Leica, Nussloch, Germany) at $-20^{\circ} \mathrm{C}$, thaw-mounted on ITO-coated glass slides, and stored at $-80^{\circ} \mathrm{C}$ until matrix application and MSI analysis.

SAMPLE PREPARATION

An automated TM-Sprayer (HTX Technologies, LLC, Chapel Hill, USA) was used for application of lipid standards between 0.01 and $0.1 \mathrm{~g} / \mathrm{L}$ concentrations in 2:1 $\mathrm{CHCl}_{3}: \mathrm{MeOH}$ onto clean ITO slides for 1 to 10 layers using the following 
protocol: spray flow rate $0.12 \mathrm{~mL} / \mathrm{min}, 30 \mathrm{~s}$ drying time between layers, at $30{ }^{\circ} \mathrm{C}$. This created concentrations from 0.33 to $3.3 \mathrm{ng} / \mathrm{mm}^{2}$ (assuming equal dispersion). Samples were then treated equivalently to brain tissue sections, where matrix was applied by sublimation: $40 \mathrm{mg}$ of DHB for $4 \mathrm{~min}$ at $160{ }^{\circ} \mathrm{C}$ with a pressure of $<4 \times$ $10^{-5}$ bar.

Hematoxylin and eosin (H\&E) staining was performed after MALDI imaging. Matrix was removed from tissue by immersion in 70\% ethanol for $3 \mathrm{~min}$. A standard H\&E protocol was then used $(95 \% \mathrm{EtOH}, 70 \% \mathrm{EtOH}, \mathrm{H} 2 \mathrm{O}$ for $30 \mathrm{~s}$ each; haematoxylin for $3 \mathrm{~min}$; $\mathrm{H} 2 \mathrm{O}, 70 \% \mathrm{EtOH}, 95 \% \mathrm{EtOH}$ each for $30 \mathrm{~s}$; eosin for $1 \mathrm{~min}$; $95 \%$ and $100 \% \mathrm{EtOH}$ for $30 \mathrm{~s}$ each; xylene for $2 \mathrm{~min}$ ). High-resolution optical images of stained tissues were generated using an Aperio CS2 digital pathology slide scanner (Leica Biosystems, Wetzlar, Germany).

\section{MASS SPECTROMETRY IMAGING InSTRUMENTATION}

Tissue sections were analyzed using a prototype $\mu$ MALDI source mounted to a Waters SYNAPT HDMS G2-Si system (Waters Corporation, Manchester, UK), as previously described by Barré et al.[382] Samples were analyzed in continuous raster mode using Waters Research Enabled Software (WRENS) to operate at 5 pixels/s, laser repetition rate of $1500 \mathrm{~Hz}$, pixel size set to $50 \mu \mathrm{m}$, MS/MS isolation window set to $\pm 1.5 \mathrm{~m} / \mathrm{z}$, and detection set to sensitivity mode (mass resolution: $\sim 15,000)$. The laser spot size was approximately $15 \times 15 \mu \mathrm{m}$. Operation of the TWave was optimized to generate the highest intensity of selected diagnostic fragments without depleting the precursor signal (wave velocity $1000 \mathrm{~m} / \mathrm{s}$ and wave height $38 \mathrm{~V}$ ). Optimization of the ion mobility traveling wave parameters allows detection down to $4.4 \mathrm{fg} / \mu^{2}$. The trap collision energy was varied according to phospholipid class from $20-35 \mathrm{~V}$ while the transfer cell was set to $2.0 \mathrm{~V}$.

\section{IN-LINE OZONE GENERATION}

Ozone generation and delivery to the instrument are as described previously.[380] Briefly, ozone was produced with a high-concentration ozone generator (TG-40; Ozone Solutions, Hull, IA, USA) from UHP oxygen (5.0 grade, 20 psi@0.4 slm; Linde Gas Therapeutics Benelux bv, Eindhoven, The Netherlands). Ozone percentage in $\mathrm{O}_{2}$ was maintained at $280 \mathrm{~g} / \mathrm{NM}^{3}$ as monitored in-line (106-H; 2B Technologies, Boulder, USA). Ozone was then leaked into the ion mobility cell gas inlet of the mass spectrometer, with the pressure maintained 
at $2.3 \mathrm{mbar}$ in the cell. Excess ozone was destroyed using a destruct catalyst (8100008; In USA, Inc., Norwood, USA). Laboratory ambient ozone concentration was monitored (106-L; 2B Technologies, Boulder, USA) and interlocked to shut off the generator if the background ozone level rose above $75 \mathrm{ppb}$.

\section{DATA ANALYSIS}

WatersRawConverter (Waters Corporation, Manchester, UK) was used to convert WRENS data using a bin size of $1 \mathrm{Da}$. Data were visualized using in-house MATLAB scripts (version R2014a, MathWorks, Natick, USA). Regions-ofinterest (ROI) were manually selected to remove off-tissue regions when plotting images. Ratio images were created with the numerator being the sum of the aldehyde and Criegee OzID fragments of a single isomer, and the denominator being the sum of the OzID fragments for all isomers. Spectra were averaged in MassLynx v4.1 and loaded into mMass software for offline recalibration and peak picking $(\mathrm{S} / \mathrm{N}=3) .100$ individual spectra were loaded into mMass to determine individual scan noise level to define the limit of detection and quantitation ( 3 and 10-fold of noise, respectively). Calibrant peaks were the most abundant OzID fragments, along with the headgroup fragment and the ozonide of the precursor lipid.

\section{LIPID NOMENCLATURE}

Lipid structure nomenclature is based on the recommendations of Liebisch et al., [396] whereby the site(s) of unsaturation are indicated by $n$-x, where $x$ is the number of carbons relative to the methyl terminus of the acyl chain. The underscore "_" or slash "/" is used for unknown or known sn-positions, respectively, of the fatty acids.

\section{RESULTS AND DISCUSSION}

We optimized the parameters of the TWIMS funnel using phospholipid standards in negative mode, finding that a wave height of $38 \mathrm{~V}$ and a wave velocity of $1,000 \mathrm{~m} / \mathrm{s}$ maximized spectral information. $\mathrm{MS}^{1}$ images of normal rat brain in negative mode were used to choose abundant single-double-bond phospholipids for optimization. PS(36:1) was found to be highly abundant with easily detected features. Increasing collisional energy prior to introduction of the ions to the TWIMS cell improves OzID functionality and sensitivity (Figure 6.1a). MS ${ }^{1}$ 
imaging of PS(36:1) highlights its abundance in the white matter of HRB tissue (Figure 6.1b). At a collisional energy (CE) of $4 \mathrm{~V}$ shows only the $n-9$ double bond isomer, following the same white/gray matter split as the $\mathrm{MS}^{1}$ ion (Figure 6.1c). Increasing CE to $35 \mathrm{~V}$, diagnostic fragment intensities increase from 2 to 100 fold and additional information relating to the fatty acid arrangement of $\operatorname{PS}(36: 1)$ is unveiled (Figure 6.1d). Improved sensitivity is sufficient to be able to image the $n$ 9 double bond isomer of the fatty acid fragment FA(18:1), which shows that it follows the same pattern as $n-9$ isomer of the intact/headgroup loss fragment (Figure 6.1e).

\section{High Collisional Energy Improves Identification}
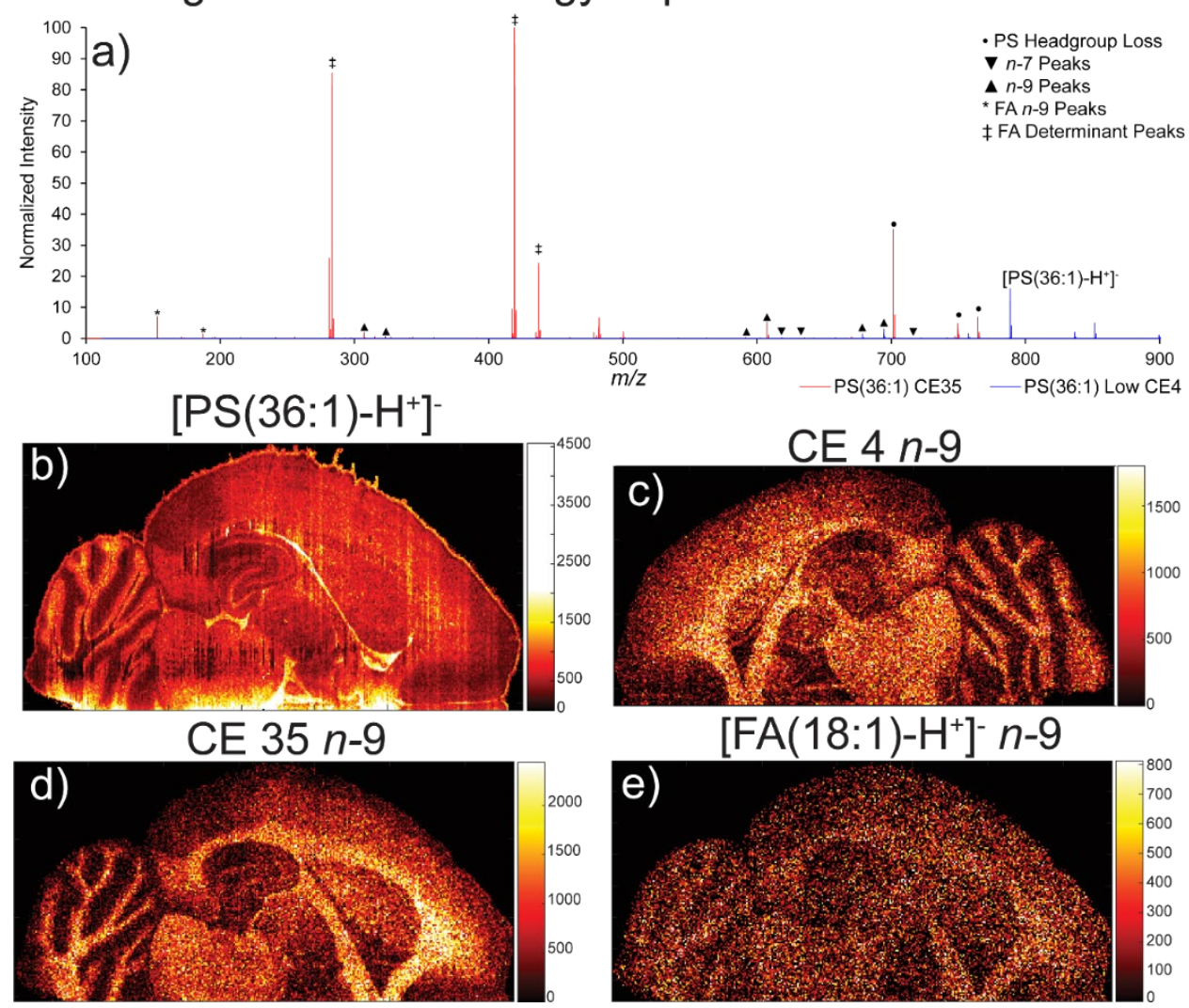

Figure 6.1. Increased collisional energy improves sensitivity. Reaction rate also improves, where the loss of the PS headgroup creates more reactive fragments, based on 1000 scans (a). Overall distribution of PS(36:1) in healthy rat brain (b), and the distribution of the only detected double-bond isomer at low collisional energy (c). High collisional energy improves sensitivity by approximately $30 \%$ (d). Further, high collisional energy enables visualization of the double bond isomer of the fatty acid fragment from the intact lipid (e). 
Simultaneously, we can determine that PS(36:1) is mainly formed of 2 acyl-chain isomers: 16:0_20:1 and 18:0_18:1, with 18:0_18:1 being the vast bulk of PS(36:1) in the brain (Figure SI 3). The split between 18:0_18:1 and 16:0_20:1 is interesting, as it follows a similar trend to the $n-7 / n-9$ trend, with 16:0_20:1 being a greater proportion of the total PS(36:1) outside of the gray matter and highlighting the hypothalamus and the ventricle. Unfortunately, the lack of CID/OzID makes us unable to distinguish between 18:0/18:1 and 18:1/18:0, nor can we explicit determine whether the $n-9$ is not, in fact, $n$-11 from FA 20:1. However, we make the assumption generally that due to the ratio of 18:0_18:1 to 16:0_20:1 implies that the vast bulk of fragment ions point towards this being $n-9$ from 18:1, rather than $n-7$ from 20:1. 

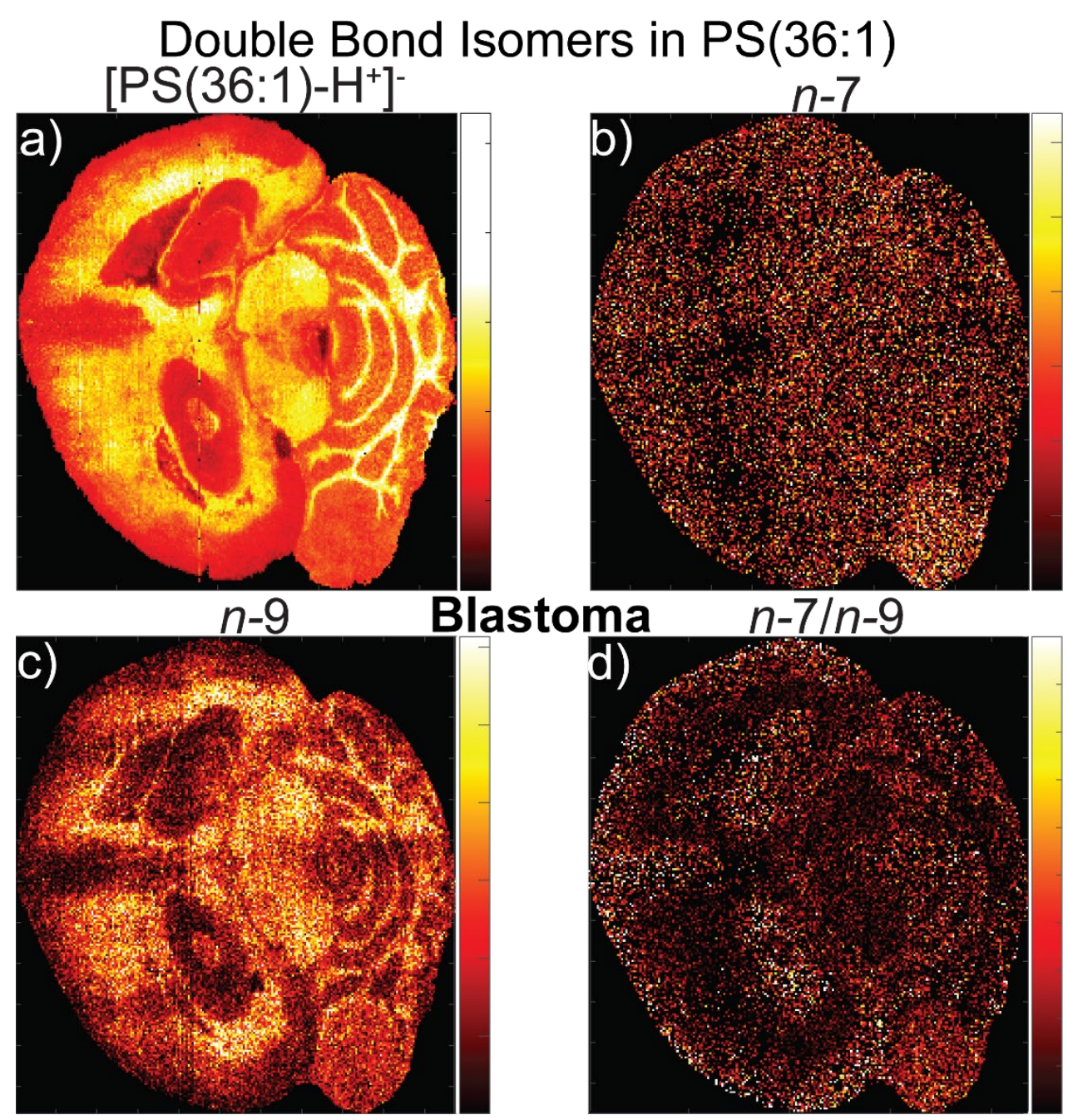

Figure 6.2. Double bond isomers of PS(36:1) in mouse brains bearing glioblastoma. MS ${ }^{1}$ imaging shows an increased presence in white matter, and decreased presence in the tumor tissue (a). In contrast to healthy rat brain, $\mathrm{n}-7$ is visible in the mouse brain, and is upregulated in the tumor (b). n-9 double bond isomer follows primary ion, being upregulated in the white matter (c). Ratio imaging of n-7 over n-9 highlights n-7 increase in the gray matter and tumor tissue $(\mathrm{d})$.

Partially, this assumption is bolstered by the fact that pure $n-7$ isomers of PS(36:1) appears to be very low in all tested tissues. BMB have PS(36:1) distributions that follow the same pattern as in healthy rat brain tissue, being highlighted in the white matter (Figure 6.2a). However, an imageable amount of the $n-7$ isomer can be detected in BMB and is slightly highlighted in the blastoma and gray matter (Figure 6.2b). The $n-9$ isomer follows the trend of the base PS(36:1) 
peak (Figure 6.2c), and the isomer ratio of $n-7$ over $n-9$ highlights both the gray matter (which is lower in overall intensity for PS(36:1)) and the tumor in the cerebellum (Figure 6.2d), which correlates with the pure $n-7$ image. We find as well that the $n-7 / n-9$ ratio matches with the ratio of PC $(36: 1)$, which has been examined previously[366].

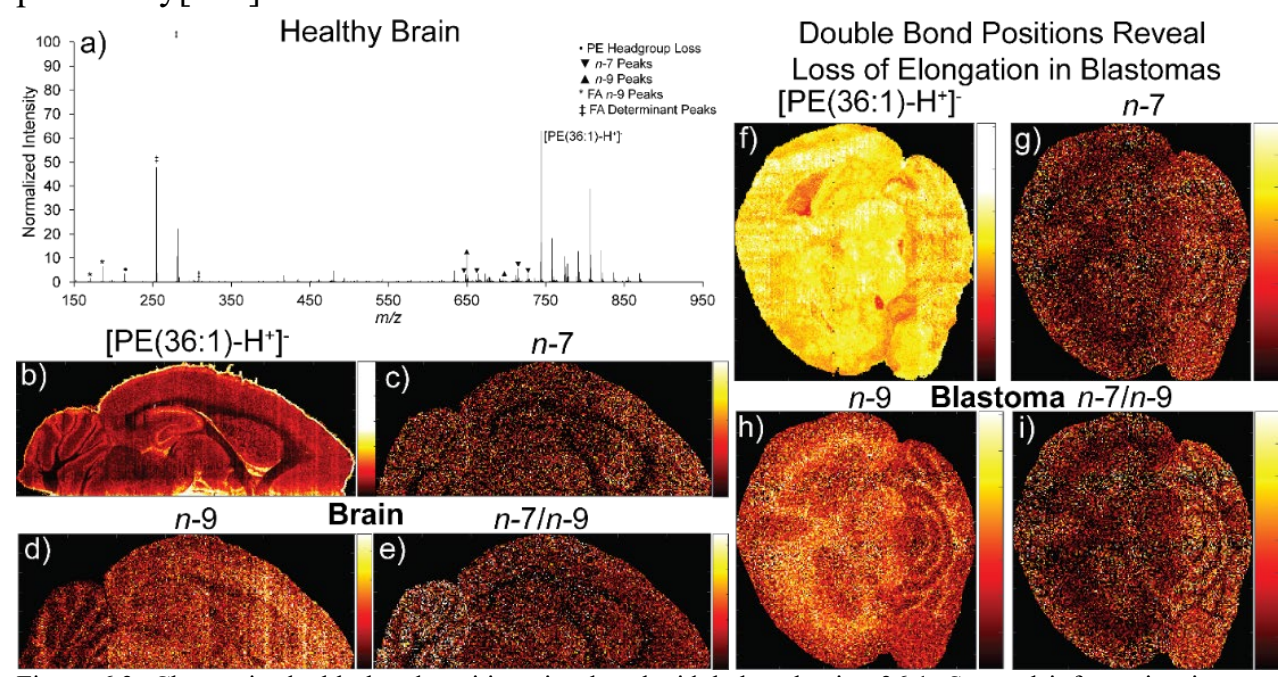

Figure 6.3. Change in double bond positions in phosphatidylethanolamine $36: 1$. Spectral information is more complex than normal due to increased presence of nitric acid adducts (a). Total signal for [PE(36:1)- $\mathrm{H}+]$ highlights generally in the gray matter, as well as in the ventricle lining and the granular cell layer of the cerebellum (b). The n-7 double bond isomer appears upregulated in the gray matter and is less abundant in the white (c). By contrast, the n-9 isomer is more abundant than the n-7 isomer and appears to highlight the molecular layer in the cerebellum (d). Ratio imaging of the n-7 to n-9 isomer shows an increase in the cerebellum, but a decrease in the specific Purkinje cell layer (e). Tumor-bearing mice brains show uniform PE(36:1) signal, being only decreased in the ventricle (f). In contrast to the rat brain, the $\mathrm{n}-7$ isomer highlights visibly in the cerebellum of the mouse (g), while the n-9 isomer highlights specifically the white matter (h). Ratio imaging also shows an increase of the n-7 isomer in the tumor region and the gray matter (i).

Imaging of $\left[\mathrm{PE}(36: 1)-\mathrm{H}^{+}\right]^{-}$shows the presence of both $n-7$ and $n-9$ isomers in both HRB and BMB with a preference for the $n-9$ isomer (Figure 6.3a). Overall assignment of fragment species is more complex in the phosphatidylethanolamine, due to the presence of significant nitric acid adduct peaks within the spectra. We attribute this to the presence of trace amounts of water within the TWIMS cell combining with ammonia (from the fragmentation of the PE headgroup) and ozone. The degeneration of signal across multiple peaks dampens imaging quality, but each individual fragment that corresponds to the same isomer produces similar images. The images in Figure 6.3 are built from the sum of all degenerate signal peaks to produce the most informative images. 
The distribution of $\mathrm{PE}(36: 1)$ appears distinct in both species, with HRB showing upregulation of $\operatorname{PE}(36: 1)$ in the dentate gyrus, in the tissue lining the ventricle, and within the molecular layer of the cerebellum Figure 6.3b). By contrast, BMB shows high levels of $\mathrm{PE}(36: 1)$ in all brain tissues and decreased in the ventricle (Figure 6.3f). Both tissues show an increase in the relative abundance of the $n-7$ isomer in the non-molecular tissues of the cerebellum, with BMB showing upregulation of the $n-7$ isomer in the tumor region (Figure 6.3e and i).

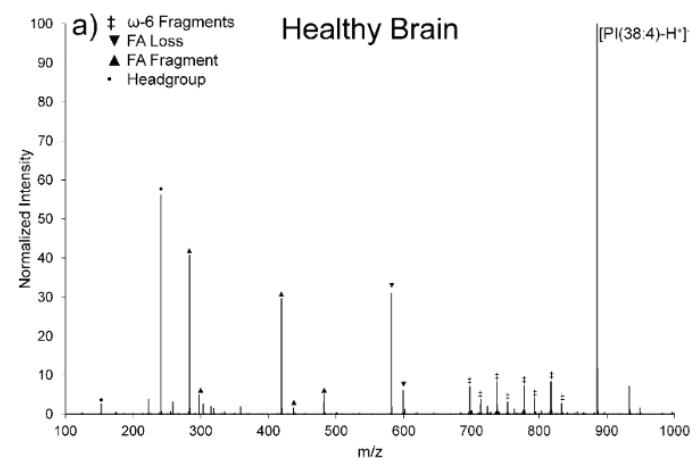

Single Double Bond Isomer of $\mathrm{PI}(38: 4)$
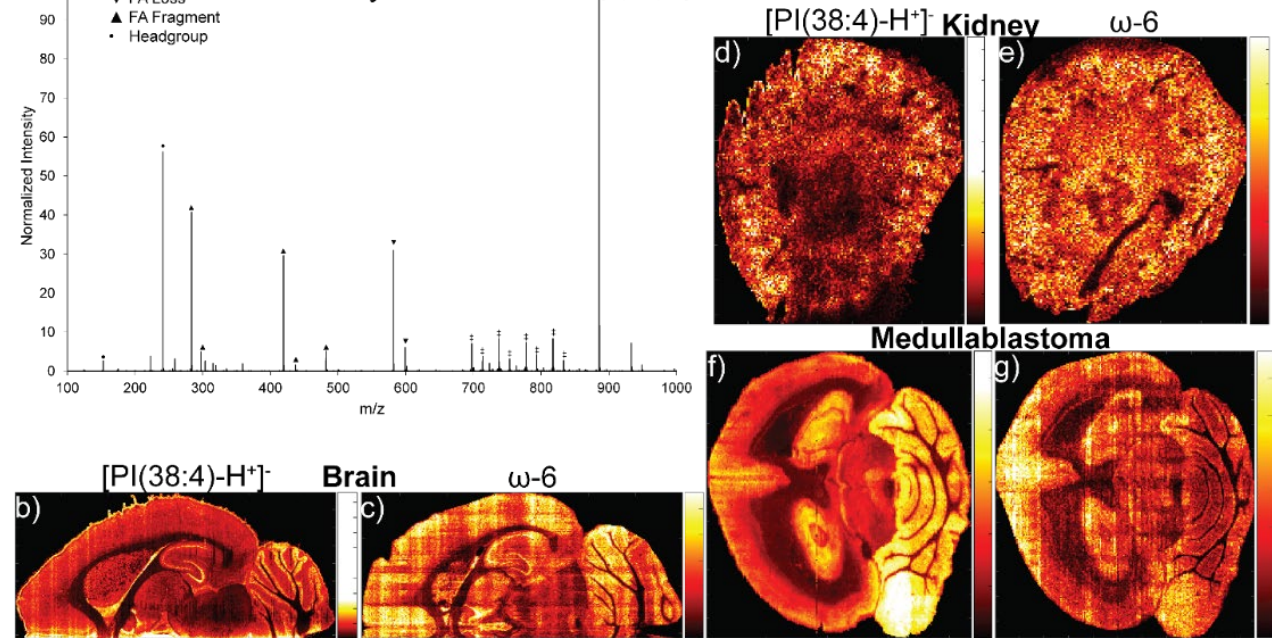

Figure 6.4. PUFA phospholipids show low isomerization of double bonds. Summed spectra of healthy brain show no signs of non- $\omega 6$ fatty acids (a). Rat brain imaging of [PI(38:4)-H+]- shows high abundance of the species in the ventricle lining, the dentate gyrus, and the Purkinje cell layer (b). Imaging of the summed $\omega-6$ fragments confirms the same distribution (c). Kidney imaging shows an increase of PI(38:4) in the cortex and inner stripe of the outer medulla, with depletion in the inner medulla and outer stripe of the outer medulla(d). $\omega-6$ fragment imaging shows high abundance in the same areas (e). Blastoma-bearing mouse brains show significant upregulation in tumor tissue, as well as generally in the cerebellar gray matter (f), which is supported by similar findings in the $\omega-6$ fragments $(\mathrm{g})$.

As we found previously with phosphatidylcholines in positive mode, the polyunsaturated fatty acid (PUFA) phospholipids display no variety in double bond position (Figure 6.4a). In the case of $\mathrm{PI}(38: 4)$, the only db-positional isomer we find is the $\omega-6$ fatty acid, which when combined with the FA information from COzID establishes it as arachidonic acid (FA 20:4). HRB shows highlights of $\mathrm{PI}(38: 4)$ in the granular layer, the dentate gyrus, and lining the ventricle (Figure $6.4 \mathrm{~b})$. The $\omega-6$ fragments generally align with this interpretation, being highlighted in the white matter, dentate gyrus, and lining the ventricle (Figure 6.4c). However, 
the cerebellum seems to be more broadly enhanced, rather than distinctly focused on the granular layer.

MK PI(38:4) is upregulated in the cortex, and to a lesser extent within the inner stripe of the outer medulla (ISOM) (Figure 6.4d). PI(38:4) is specifically downregulated within the medulla itself, and towards the renal pelvis. $\omega-6$ imaging

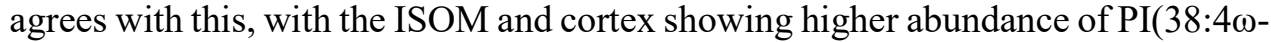
6) (Figure 6.4e). Being removed from the $\mathrm{MS}^{1}$ image, there is a smaller section of the renal pelvis available in the COzID image, but there still appears to be a reduction in the abundance of $\mathrm{PI}(38: 4)$ visible beneath the fracture in the kidney section, which corresponds to the pelvis.

BMB is quite different from HRB, with the white matter of the cerebellum being much more strongly upregulated than the white matter of the rest of the brain (Figure 6.4f). Within the blastoma of the BMB, PI(38:4) is extraordinarily abundant, a result that correlates well with the increase in cellular respiration within the tumor. $\omega-6$ imaging of BMB tissue still shows this same white/gray matter split, along with an increase in the tumor region (Figure 6.4g).

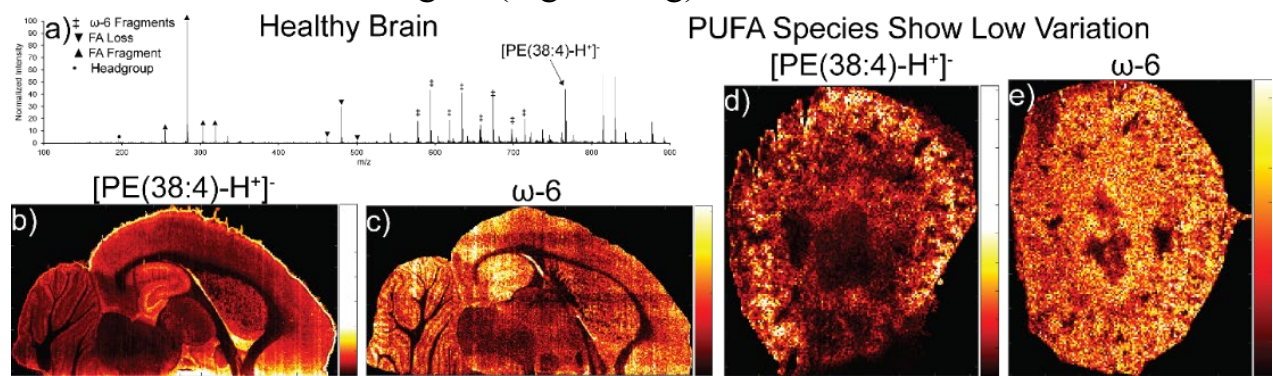

Figure 6.5. Imaging of [PE(38:4)-H+]- in rat brain and kidney. Summed spectral average of [PE(38:4)-H+]- shows presence of only PE fragments and $\omega-6$ fatty acids (a). Image of $\mathrm{PE}(38: 4)$ shows an increase in the dentate gyrus, the ventricle lining, and the molecular layer of the cerebellum (b). $\omega-6$ fatty acid fragments show upregulation similar to the primary ion (c). $[\mathrm{PE}(38: 4)-\mathrm{H}+]$ - in kidney shows complete depletion in the inner medulla and upregulation in the cortex (d). Imaging of the $\omega-6$ fragments is more ambiguous about the upregulation in the cortex vs the inner and outer stripe of the medulla, but still shows depletion in the inner medulla (e).

Like PI(38:4), PE(38:4) appears to be made of a single db-isomer, that of

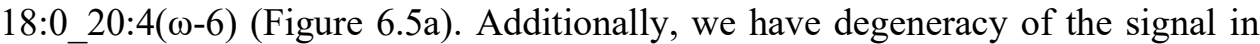
the ozonolysis fragments due to the generation of nitric acid in the TWIMS cell. The abundance of $\operatorname{PE}(38: 4)$ corresponds to the dentate gyrus, the ventricle lining, and the molecular layer of the cerebellum in HRB, the same as in PI(38:4) (Figure $6.5 b$ ). The single $\mathrm{db}$-isomer highlights the same brain tissue, with no contaminating peaks visible to confound identification (Figure 6.5c). MK imaging of $\mathrm{PE}(38: 4)$ 
shows high abundance in the cortex, and depletion in the inner medulla, which correlates with PI(38:4) (Figure 6.5d). The $\omega-6$ fragments are depleted in the inner medulla, which matches with the primary ion, but the overall difference between the stripe of the medulla and the cortex is lessened, we think as a result of the degeneracy of signal interfering with smooth imaging of the difference in abundance (Figure 6.5e).

While the lack of true CID/OzID increases the difficulty in making true isomerically-resolved lipid images, COzID still allows us to differentiate between isobaric lipids. $\left[\mathrm{PG}(34: 1)-\mathrm{H}^{+}\right]^{-}$and $\left[\mathrm{PA}(40: 6)-\mathrm{H}^{+}\right]$differ by $21 \mathrm{mDa}$, making them distinguishable in high resolution mass spectrometers, but not on our current platform ( $\sim 35,000$ vs 15,000 resolving power). However, with COzID we can distinguish between them based on the acyl chains that they are comprised of, while still identifying double bond isomers (Figure 6.6a). Similarly to the arachidonic

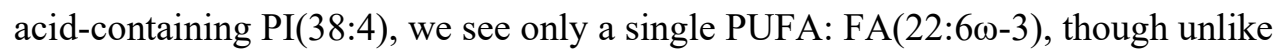
for either PS(36:1) or PE(36:1), we can see both MUFA $n-7$ and $n-9$. Imaging of the $\mathrm{MS}^{1}$ ion shows slight increases in the molecular layer of the cerebellum in HRB, as well as within the dentate gyrus (Figure 6.6b). Imaging of the $\omega-3$ fragments contrastingly highlights the granular layer of the cerebellum, as well as the gray matter of the brain as a whole (Figure 6.6c), consistent with previous findings.

Investigation of the $n-7$ isomer of $\mathrm{PG}(34: 1)$ in HRB shows similar morphology to the primary ion image, but more exaggerated in upregulation (Figure 6.6d). The molecular layer is more readily apparent, as well as the lining of the ventricle in the forebrain, with low or no abundance in the white matter. The lower abundance $n-9$ isomer seems to have no particularly distinct features, save that it is not found in the white matter (Figure 6.6e). This greater abundance of $n-7$ vs $n-9$ in PG (34:1) is interesting, as it is the opposite of what is found in the $36: 1$ phospholipids, whether PS or PE.

Precursor imaging of the combined $\left[\mathrm{PG}(34: 1)-\mathrm{H}^{+}\right]^{-}$and $\left[\mathrm{PA}(40: 6)-\mathrm{H}^{+}\right]^{-}$in MK shows upregulation within the cortex of the kidney, and relative downregulation in the medulla and renal pelvis (Figure 6.6f). Similarly to the HRB, where the main component of the precursor is PA(40:6), in MK the $\omega-3$ is abundant within the cortex, while being depleted within the medulla. However, overall sensitivity is lower in MK than HRB, which combines poorly with the significantly degenerate signal of PA(40:6), leading to a less conclusive distribution image than is found in HRB. $\mathrm{PG}(34: 1)$ in MK is highly enriched in the $n-7$ isomer, which is 
upregulated in the medulla of the kidney (Figure 6.6h). The $n-9$ isomer is low enough in abundance that drawing conclusive evidence of specific distribution is impossible in our current instrumentation (Figure 6.6i).

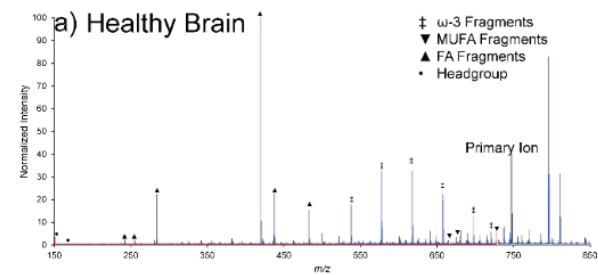

$\left[P G(34: 1)-\mathrm{H}^{+}\right]-\left[\mathrm{PA}(40: 6)-\mathrm{H}^{+}\right]$
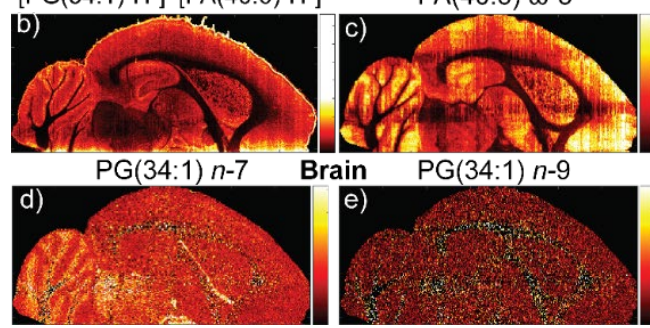

Differentiating Isobaric Lipids by Double Bond Fragments

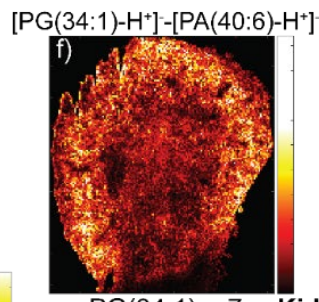

$\operatorname{PA}(40: 6) \omega-3$
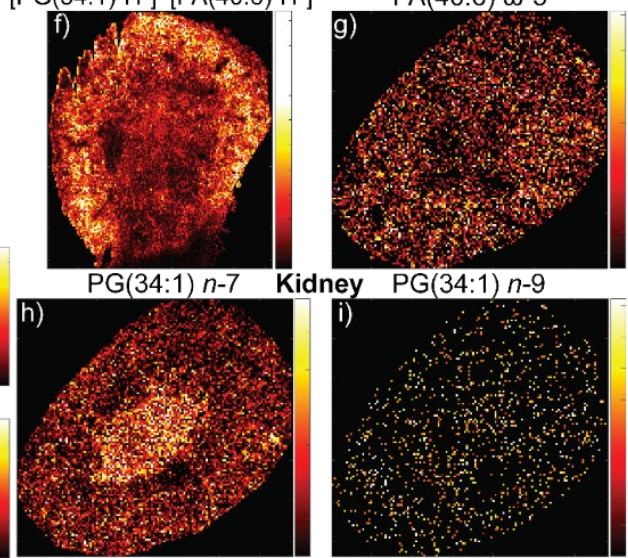

Figure 6.6. Differentiating between isobaric lipids and isomeric lipids simultaneously using COzID. Spectral complexity is heightened by isobaric interferences, but CID and OzID fragments differentiate between species (a). Unresolved imaging of [PG(34:1)-H+]- and [PA(40:6)-H+]- shows no highlights other than depletion in white matter of the brain and an indeterminate increase in the molecular layer (b). However, PA(40:6) $\omega-3$ fragments highlights the granule cell layer of the cerebellum, and the gray matter of the cerebrum (c). Imaging of the n-7 isomer of PG(34:1) highlights the molecular layer, as well as the ventricle lining (d). The n-9 isomer of PG(34:1) shows no highlight except for spotty increases in the pia mater (e). Unresolved imaging of [PG(34:1)-H+]- and [PA(40:6)-H+]- in mouse kidney shows upregulation in the cortex (f). This corresponds to high levels of the $\omega-3$ fragments of $\mathrm{PA}(40: 6)$ (g), but not to the n-7 isomer of PG(34:1), which is found mainly in the medulla (h). The $\mathrm{n}-9$ isomer is too low in abundance to clearly distinguish any areas of upregulation (i).

\section{CONCLUSION}

The expansion of OzID-MSI into negative mode shows distinct distributions of double bond isomers dependent on the phospholipid in comparison to previous work[366], with no apparent variation in the PUFA phospholipids. While CID/OzID is not available in negative mode, owing to the lack of ringformation as in the metal cationized phosphatidylcholines, it can be used in combination as COzID, allowing for the simultaneous investigation of both double bond positional isomers and acyl-chain variations. In particular, while this combination does not allow for specific assignment of lipids to stereonumber, robust information from both ozonolysis and collisional fragmentation can allow for the assignment of specific double bonds to particular acyl chains, enabling identification of all levels except double bond geometry and stereonumbering. 
The tissues tested show the applicability of this technique to multiple imaging experiments, in both healthy and diseased tissue. Further, specific tissue types can be identified through their double bond positional isomers in multiple phospholipid classes, and changes between phospholipid classes can be identified, which has thus far been impossible in purely positive-mode mass spectrometry imaging experiments. We see this as a new tool in the arsenal of mass spectrometry imaging, which can be used to enhance the overall understanding of both healthy and diseased tissue beyond what has thus far been shown. 


\section{Chapter 7 VALORIZATION}


Defining the overall impact of a work like this thesis is a difficult undertaking. As a whole, science is an interconnected web of ideas, experiments, and collaborations, advancing in 1,000 different directions simultaneously. Combining this general trend within the sciences with the ever-increasing drive towards complete omics awareness in biological systems, it becomes obvious that no one scientist can cover every avenue in understanding a biological question. This work has pushed forward that envelope by combining technological innovation in-house with biological questions from abroad, attempting to answer their specific questions.

\section{SCIENTIFIC IMPACT}

Perhaps the easiest effect to measure from this thesis is the number of collaborative efforts that have spun out from it. Ultra-high mass resolving power combined with mass spectrometry imaging (Chapter 2) lead directly to the National High Magnetic Field Laboratory (Tallahassee, USA) to purchase the same MALDI source for their instruments. This greatly expands the capabilities of that institute and enables many more people the opportunity to explore isobaric compounds in general, as well as the chance to expand further in the capabilities of that system, either in ultraviolet photodissociation or other MS/MS methodologies. As the MagLab is a national laboratory in the United States that all scientists can have access to, this is a wonderful step forward for many people to employ techniques with machinery they otherwise would not.

Building upon the utility of MALDI-2, both in its ability to increase spectral information and its power to image smaller pixels has seen utility in understanding more of the causes in multiple sclerosis. Initial work with the University of Hasselt (Hasselt, Belgium) exposed the presence of Stearoyl-CoA desaturase-1 in lesions of MS. Further information was unveiled in the process of Chapter 3, which has prompted the University of Hasselt to deepen its ties to M4I to further explore the unusual lipidomic alterations unveiled by post-ionization and single cell imaging. Similarly, the Institute of Bioengineering, Ecole Polytechnique federale de Lausanne (Switzerland) utilized this technology to investigate single cell lipidomics in fibroblasts. The Technical University of Munich has developed an on-going collaboration with M4I centered around the understanding of nonalcoholic steatohepatitis in both preclinical mouse models and patient tissue, which is enhanced by the use of MALDI-2 and has unveiled not just fatty acid 
changes but specific localization of apoptosis byproducts in the liver. Both the School for Mental Health and Neuroscience (Maastricht, Netherlands) and the University of Gothenburg (Gothenburg, Sweden) have looked to M4I to continue developing our understanding of Alzheimer's disease, and the dyslipidemia that associates with the plaques that are a hallmark of Alzheimer's. Multiple sclerosis, Alzheimer's, nonalcoholic steatohepatitis, these diseases are merely the first set of projects that can be improved by the application of MALDI-2, and the expansion of that technology to new instruments and coupled with different capabilities will only see ever finer understanding of biology.

Shifting away from MALDI-2, the combination of OzID with MSI has shown great potential in studying prostate cancer. The Queensland University of Technology (Brisbane, Australia) has partnered with M4I for the ability to study the global context of cancer progression in human tissue. CID/OzID imaging has already been demonstrated before, but the more difficult double bond isomers have yet to be studied in great depth. However, as demonstrated in Chapters 4 and 5, it is now possible to study these in not just the commonly investigated phosphatidylcholines, but many more phospholipids, as well. As all of the lipid classes are interconnected, but operate with different synthetic pathways, the ability to analyze all of them deepens our ability to understand the whole state of a biological tissue.

\section{COMMERCIAL IMPACT}

Through the duration of this thesis, I had the privilege of working with several companies in the pursuit of improving mass spectrometry imaging. Bruker Daltonics (Bremen, Germany), Molecular Horizons, S.R.L. (Brettona, Italy), and Spectroglyph, LLC. (Kennewick, USA) all provided unique opportunities to extend the principles developed in this thesis to wider audiences. Working with Bruker enabled me to apply the expertise I have gained in custom software and hardware packages to a format that is applicable to the routine user, including MALDI-2 and, potentially, data-dependent acquisitions. These data-dependent acquisitions can be read through the auspices of Molecular Horizons' LipostarMSI, a software package that I had the great joy of helping design. Being a vendor-neutral platform, LipostarMSI is useful to scientists working with any commercial instrument and can interpret the complex data derived from data-dependent acquisition experiments (a la Chapter 3). Significant use of Spectroglyph's MALDI ion source 
for Thermo instruments has also helped refine both physical and software features available from that company.

My contribution to many of these commercial endeavors was as an end user. By offering active feedback on these systems, I have helped make stronger, more robust systems that provide stable platforms for further research. In turn, these companies have helped show me how to constrain the enthusiasm of research to the restrictions of feasibility.

\section{SOCIETAL IMPACT}

Perhaps the greatest showing for the societal impact of this work is in the generation of a magazine article about a scientific paper that this work helped publish. The Nederlandse Vereniging van bioMedisch Laboratoriummedewerkers (NVML) asked for an article to be written based on the Nature published article "Auto-aggressive CXCR6+ CD8 T cells cause liver immune pathology in NASH," to translate the findings of that work for medical clinicians. That such a magazine article would be requested immediately after the publishing of that journal article points to how impactful this type of research can be, and how necessary it is to make this accessible to more people.

The desire to understand the complete spatial context of tissues is something that will resonate for decades, in my opinion. This work is simply a driving force in making mass spectrometry imaging a vital portion of spatial omics. By providing techniques that can be applied to old systems, programs that can be applied in a vendor-neutral fashion, and showing the utility of combining extant systems, it is my fervent desire that many more people will be able to benefit. 


\section{Chapter 8 CONCLUSION}


The summation of this work is an attempt to push the boundaries of what is possible with mass spectrometry imaging, and to combine those efforts with others working in related fields to create spatial omics. As has been shown repeatedly, both within this work and within the mass spectrometry imaging community is that spatial awareness matters. The spatial context allows for both better understanding of the underlying biological context and for the ability to detect minute changes that are invisible in aggregate. The development of powerful, reproducible methods that can identify specific lipids is a key factor in forging better spatial omics.

\section{Biological ConteXt In MASS SPECTROMETRy IMAgING}

Functionally, the chapters of this thesis revolve around improvements to lipid identification in mass spectrometry imaging. Evolving developments in MSI have pushed forward lipids as both easy targets for ionization and as important biomolecules for their multiple roles in biological function. Emerging technologies have been employed to Chapter 1, however, highlights the idea that while lipids are convenient for the identification of disease or metabolic disorders, they are typically an end-point or substrate in these dysfunctions. Their utility cannot be understated, but they are not able to fully encompass the scope of a tissue. It is necessary to look beyond the lipids to the enzymatic processes that produce them. That aside, for processes where the underlying biological process is wellunderstood, improvements in spatial-resolution and isomer-resolution of lipids are the next goal in lipidomics.

\section{UltRA-High RESOLUTION}

Improvements in mass resolution is a powerful tool in the kit of lipidomics. Ultra-high mass resolving power, as described in chapter 3, allows for the direct separation and sum-composition identification of lipids. The mass accuracy of $<100 \mathrm{ppb}$ in the lipid mass range, with separation potential sufficient to distinguish between many common isobars past $\mathrm{m} / \mathrm{z} 1,000$ makes it a superlative mass spectrometer. In these experiments, we showed resolution of features as closely isobaric as $1.78 \mathrm{mDa}$, allowing identification of more than 2,000 lipid related peaks that were abundant enough to generate imaging information. The stability of the experimental device allowed for images that needed no normalization to show accurate representations of the ratio of lipid abundance. 
However, it has 2 failings. First, the speed of acquisition is still low, forcing either small sections of tissue to be examined or long experimental times. Neither of these is particularly compatible with mass spectrometry imaging, as sample degradation (whether due to samples being maintained at atmosphere or from matrix sublimation) becomes a problem with longer experiment time while instrument time can be a previous commodity. This is especially true for the instrument used in this experiment, as there are only 2 of them in the world, making it difficult for most people to use. Second, the data collected is only $\mathrm{MS}^{1}$ data, which allows for highly accurate sum-composition identification of lipids, but precludes any identification of either acyl chain composition or double-bond positional isomers. The inclusion of either capability would greatly improve the utility of this instrumental setup.

\section{High Spatial Resolution}

The combination of MALDI-2 with oversampling in MALDI shows great utility in finding highly specific spatial location of lipids. More lipids can be identified while great oversampling in MALDI-2 than can be found in normal MALDI experiments without any oversampling. Further, the use of MALDI-2 ionizes species that are either normally invisible in MALDI imaging, or that are only available in a specific ion detection mode (e.g., positive or negative mode). Both of these advantages are available without changing the primary laser configuration.

This experiment has a minor design flaw in that the oversampling of an ovoid beam does not preclude the ionization of lipids from the entire laser footprint, potentially confounding or obscuring the exact spatial location of the analytes of interest. Further, the shape of the resultant pixels are not true squares, but instead are effectively crescent-moons that are further subdivided into crescents. While these are approximated as squares, as the spatial resolution of the pixels increases, the exact shape of the pixels will become more important, as will the desire to preclude any signal that might occur from laser desorption ionization from the previously desorbed surface.

\section{OZONE-INDUCED DisSOCIATION}

The application of ozone to identify double-bond isomers is not unique to this pair of studies. However, the speed of acquisition and the sensitivity of the 
instrumentation is one to two orders of magnitude greater than any previous imaging study. The nature of the instrument, which necessitates the combined CID, OzID, CID/OzID, and CID/OzID ${ }^{2}$ fragments all in a single experiment is a problem to be addressed, as having all of the fragments from each pathway combined together clouds the absolute identification of some lipids.

Negative mode OzID has never been shown before in an imaging modality, making this endeavor as perhaps one of the most significant, at least in terms of application to greater experimentation. While studies have shown the changes that occur within the phosphatidylcholines, those that show the other phospholipids are much rarer, and a source for significant improvement in lipidomics. 


\section{Chapter 9 SUMMARY}


Understanding in spatial omics requires two intertwined but distinct levels of knowledge: precise, resolved ion images and accurate identification of analytes. These thesis addresses both levels to improve lipidomics assignment of tissues.

Chapter 1 offers a brief overview of the issues facing mass spectrometrists in the study of lipids. Different analytical methods are discussed, including MALDI, SIMS, and DESI, and the different advantages and disadvantages of these relating to mass spectrometry imaging. Several applications are then investigated, which are then related to further dimensions that can be added to experimental models. Lastly, future potential developments are discussed.

Chapter 2 focuses on the utility of ultra-high mass resolving power to improve lipid identification. The application of a MALDI imaging source to a 21 Tesla FT-ICR is described, as is the steps to produce intelligible results from ultrahigh resolution data. Both mass accuracy and dynamic range are discussed, and several images of known lipids are described.

In chapter 3 MALDI-2 oversampling is shown to produce significant improvements in spatial resolution without MALDI beam modification. The basic process of oversampling is described in the course of identifying the maximum oversampling possible with this instrumentation. Several examples of different tissue types are displayed that show distinctive morphological or analytic changes unavailable in normal MALDI.

Chapter 4 describes the development of ozone-based lipid identification on a Synapt G2Si instrument. The modifications required for operation are laid out, followed by comparison of this instrumentation to earlier OzID-MSI platforms. Enhancements in sensitivity and speed of at least 2 orders of magnitude are demonstrated, as well as several types of experiment that were previously unavailable.

Chapter 5 unveils the first use of OzID in negative ionization mode in combination with MSI. Several of the advantages and disadvantages of negativemode OzID are discussed, including the lack of true CID/OzID. Phospholipids of 5 different classes are described in detail in 3 different tissue types, representing healthy, dysfunctional, and tumor-bearing modalities. 


\section{Chapter 10 SAMENVETTING}


Inzicht in ruimtelijke omics vereist twee met elkaar verweven maar verschillende kennisniveaus: nauwkeurige, opgeloste ionenbeelden en nauwkeurige identificatie van analyten. Dit proefschrift behandelt beide niveaus om de toewijzing van lipidomics aan weefsels te verbeteren.

Hoofdstuk 1 biedt een kort overzicht van de problemen waarmee massaspectrometristen worden geconfronteerd bij de studie van lipiden. Verschillende analysemethoden worden besproken, waaronder MALDI, SIMS en DESI, en de verschillende voor- en nadelen hiervan met betrekking tot massaspectrometriebeeldvorming. Vervolgens worden verschillende toepassingen onderzocht, die vervolgens worden gerelateerd aan verdere dimensies die aan experimentele modellen kunnen worden toegevoegd. Tot slot worden mogelijke toekomstige ontwikkelingen besproken.

Hoofdstuk 2 richt zich op het nut van ultrahoog massa-oplossend vermogen om de identificatie van lipiden te verbeteren. De toepassing van een MALDI-beeldbron op een 21 Tesla FT-ICR wordt beschreven, evenals de stappen om begrijpelijke resultaten te produceren op basis van gegevens met ultrahoge resolutie. Zowel massanauwkeurigheid als dynamisch bereik worden besproken, en verschillende afbeeldingen van bekende lipiden worden beschreven.

In hoofdstuk 3 wordt aangetoond dat MALDI-2 oversampling significante verbeteringen in ruimtelijke resolutie produceert zonder MALDI-straalmodificatie. Het basisproces van overbemonstering wordt beschreven tijdens het identificeren van de maximaal mogelijke overbemonstering met deze instrumenten. Er worden verschillende voorbeelden van verschillende weefseltypes weergegeven die kenmerkende morfologische of analytische veranderingen vertonen die niet beschikbaar zijn in normale MALDI.

Hoofdstuk 4 beschrijft de ontwikkeling van op ozon gebaseerde lipide-identificatie op een Synapt G2Si-instrument. De wijzigingen die nodig zijn voor de werking worden uiteengezet, gevolgd door vergelijking van deze instrumentatie met eerdere OzID-MSIplatforms. Verbeteringen in gevoeligheid en snelheid van ten minste 2 ordes van grootte worden aangetoond, evenals verschillende soorten experimenten die voorheen niet beschikbaar waren.

Hoofdstuk 5 onthult het eerste gebruik van OzID in negatieve ionisatiemodus in combinatie met MSI. Een aantal van de voor- en nadelen van OzID in negatieve modus worden besproken, waaronder het ontbreken van echte CID/OzID. Fosfolipiden van 5 verschillende klassen worden in detail beschreven in 3 verschillende weefseltypes, die gezonde, disfunctionele en tumordragende modaliteiten vertegenwoordigen. 


\section{Chapter 11 REFERENCES}


1. $\quad$ Gold, V., The IUPAC Compendium of Chemical Terminology. 2019.

2. Berg, J.M., J.L. Tymoczko, and L. Stryer, Fatty Acid Metabolism, in Biochemistry. 2002, W. H. Freeman and Company: New York.

3. Zechner, R., et al., Lipolysis: pathway under construction. Curr Opin Lipidol, 2005. 16(3): p. $333-40$.

4. Gault, C.R., L.M. Obeid, and Y.A. Hannun, An overview of sphingolipid metabolism: from synthesis to breakdown. Adv Exp Med Biol, 2010. 688: p. 1-23.

5. Vaughan, M. and D. Steinberg, Glyceride biosynthesis, glyceride breakdown and glycogen breakdown in adipose tissue: mechanisms and regulation, in Comprehensive Physiology. 2011.

6. Brown, D.A. and E. London, Structure and function of sphingolipid-and cholesterol-rich membrane rafts. J Biol Chem, 2000. 275(23): p. 17221-4.

7. Green, D.R., Apoptosis and sphingomyelin hydrolysis. The flip side. J Cell Biol, 2000. 150(1): p. F5-7.

8. Hannun, Y.A. and L.M. Obeid, The Ceramide-centric universe of lipid-mediated cell regulation: stress encounters of the lipid kind. J Biol Chem, 2002. 277(29): p. 25847-50.

9. Hetz, C.A., et al., Caspase-dependent initiation of apoptosis and necrosis by the Fas receptor in lymphoid cells: onset of necrosis is associated with delayed ceramide increase. J Cell Sci, 2002. 115(Pt 23): p. 4671-83.

10. Snider, A.J., K.A. Orr Gandy, and L.M. Obeid, Sphingosine kinase: Role in regulation of bioactive sphingolipid mediators in inflammation. Biochimie, 2010. 92(6): p. 707-15.

11. Lavieu, G., et al., Regulation of autophagy by sphingosine kinase 1 and its role in cell survival during nutrient starvation. J Biol Chem, 2006. 281(13): p. 8518-27.

12. Venable, M.E., et al., Role of ceramide in cellular senescence. J Biol Chem, 1995. 270(51): p. 30701-8.

13. Spiegel, S. and S. Milstien, Sphingosine 1-phosphate, a key cell signaling molecule. J Biol Chem, 2002. 277(29): p. 25851-4.

14. Futerman, A.H., Intracellular trafficking of sphingolipids: relationship to biosynthesis. Biochim Biophys Acta, 2006. 1758(12): p. 1885-92.

15. Kannan, M., et al., Phosphatidylserine synthesis at membrane contact sites promotes its transport out of the ER. J Lipid Res, 2017. 58(3): p. 553-562.

16. Frayn, K.N., et al., Coordinated regulation of hormone-sensitive lipase and lipoprotein lipase in human adipose tissue in vivo: Implications for the control of fat storage and fat mobilization. Advances in Enzyme Regulation, 1995. 35: p. 163-178.

17. Roberts, L.D., et al., Methods for performing lipidomics in white adipose tissue. Methods Enzymol, 2014. 538: p. 211-31.

18. Nestel, P.J., Fish oil and cardiovascular disease: lipids and arterial function. Am J Clin Nutr, 2000. 71(1 Suppl): p. 228S-31S.

19. Wierzbicki, A.S., Lipids, cardiovascular disease and atherosclerosis in systemic lupus erythematosus. Lupus, 2000. 9(3): p. 194-201.

20. Emerging Risk Factors, C., et al., Lipid-related markers and cardiovascular disease prediction. JAMA, 2012. 307(23): p. 2499-506.

21. Castelli, W.P., Lipids, risk factors and ischaemic heart disease. Atherosclerosis, 1996. 124: p. S1-S9.

22. Santos, C.R. and A. Schulze, Lipid metabolism in cancer. FEBS J, 2012. 279(15): p. 2610-23.

23. Suburu, J. and Y.Q. Chen, Lipids and prostate cancer. Prostaglandins Other Lipid Mediat, 2012. 98(1-2): p. 1-10.

24. Borgquist, S., et al., Apolipoproteins, lipids and risk of cancer. Int J Cancer, 2016. 138(11): p. 2648-56.

25. Kannel, W.B., Lipids, diabetes, and coronary heart disease: Insights from the Framingham Study. American Heart Journal, 1985. 110(5): p. 1100-1107. 
26. Krauss, R.M., Lipids and lipoproteins in patients with type 2 diabetes. Diabetes Care, 2004. 27(6): p. 1496-504.

27. Georg, P. and B. Ludvik, Lipids and diabetes. Journal of clinical and basic cardiology, 2000. 3(3): p. 159-162.

28. Foley, P., Lipids in Alzheimer's disease: A century-old story. Biochim Biophys Acta, 2010. 1801(8): p. 750-3.

29. Kao, Y.C., et al., Lipids and Alzheimer's Disease. Int J Mol Sci, 2020. 21(4).

30. Di Paolo, G. and T.W. Kim, Linking lipids to Alzheimer's disease: cholesterol and beyond. Nat Rev Neurosci, 2011. 12(5): p. 284-96.

31. Emerging Risk Factors, C., et al., Major lipids, apolipoproteins, and risk of vascular disease. JAMA, 2009. 302(18): p. 1993-2000.

32. Duntas, L.H., Thyroid disease and lipids. Thyroid, 2002. 12(4): p. 287-93.

33. Trevisan, R., A.R. Dodesini, and G. Lepore, Lipids and renal disease. J Am Soc Nephrol, 2006. 17(4 Suppl 2): p. S145-7.

34. Braverman, N.E. and A.B. Moser, Functions of plasmalogen lipids in health and disease. Biochim Biophys Acta, 2012. 1822(9): p. 1442-52.

35. Xicoy, H., B. Wieringa, and G.J.M. Martens, The Role of Lipids in Parkinson's Disease. Cells, 2019. 8(1).

36. Dortland, A.K.B.V., et al., Associations Between Serum Lipids and Major Depressive Disorder: Results From the Netherlands Study of Depression and Anxiety (NESDA). Journal of Clinical Psychiatry, 2010. 71(6): p. 729-736.

37. Solter, V., et al., Elevated serum lipids in veterans with combat-related chronic posttraumatic stress disorder. Croat Med J, 2002. 43(6): p. 685-9.

38. D'Ambrosio, V., et al., Serum lipids, metabolic syndrome and lifetime suicide attempts in patients with bipolar disorder. Prog Neuropsychopharmacol Biol Psychiatry, 2012. 37(1): p. 136-40.

39. Liebisch, G., et al., Update on LIPID MAPS classification, nomenclature, and shorthand notation for MS-derived lipid structures. J Lipid Res, 2020. 61(12): p. 1539-1555.

40. Yetukuri, L., et al., Informatics and computational strategies for the study of lipids. Mol Biosyst, 2008. 4(2): p. 121-7.

41. van Meer, G., Cellular lipidomics. EMBO J, 2005. 24(18): p. 3159-65.

42. de Hoffmann, E. and V. Stroobant, Mass Spectrometry: Principles and Applications. 2013: Wiley.

43. Siuzdak, G., An introduction to mass spectrometry ionization: An excerpt from The Expanding Role of Mass Spectrometry in Biotechnology, 2nd ed.; MCC Press: San Diego, 2005. Journal of the Association for Laboratory Automation, 2004. 9(2): p. 50-63.

44. Banerjee, S. and S. Mazumdar, Electrospray ionization mass spectrometry: a technique to access the information beyond the molecular weight of the analyte. Int $\mathrm{J}$ Anal Chem, 2012. 2012: p. 282574.

45. Ivanova, P.T., et al., Glycerophospholipid identification and quantitation by electrospray ionization mass spectrometry. Methods Enzymol, 2007. 432: p. 21-57.

46. Polettini, A., et al., Implementation and performance evaluation of a database of chemical formulas for the screening of pharmaco/toxicologically relevant compounds in biological samples using electrospray ionization-time-of-flight mass spectrometry. Anal Chem, 2008. 80(8): p. 3050-7.

47. Huppertz, L.M., et al., A comprehensive library-based, automated screening procedure for 46 synthetic cannabinoids in serum employing liquid chromatography-quadrupole ion trap mass spectrometry with high-temperature electrospray ionization. J Mass Spectrom, 2014. 49(2): p. 117-27.

48. Schulze, A., et al., Expanded newborn screening for inborn errors of metabolism by electrospray ionization-tandem mass spectrometry: results, outcome, and implications. Pediatrics, 2003. 111(6 Pt 1): p. 1399-406. 
49. Heaney, L.M., D.J. Jones, and T. Suzuki, Mass spectrometry in medicine: a technology for the future? Future Sci OA, 2017. 3(3): p. FSO213.

50. Zhou, W., S. Yang, and P.G. Wang, Matrix effects and application of matrix effect factor. Bioanalysis, 2017. 9(23): p. 1839-1844.

51. Folch, J., M. Lees, and G.H.S. Stanley, A Simple Method for the Isolation and Purification of Total Lipides from Animal Tissues. Journal of Biological Chemistry, 1957. 226(1): p. 497-509.

52. Bligh, E.G. and W.J. Dyer, A rapid method of total lipid extraction and purification. Can J Biochem Physiol, 1959. 37(8): p. 911-7.

53. Sheng, J., R. Vannela, and B.E. Rittmann, Evaluation of methods to extract and quantify lipids from Synechocystis PCC 6803. Bioresour Technol, 2011. 102(2): p. 1697-703.

54. Smedes, F., Determination of total lipid using non-chlorinated solvents. Analyst, 1999. 124(11): p. 1711-1718.

55. Christie, W.W., Lipid Analysis. Vol. 207. 1982, Oxford: Pergamon Press.

56. de Boer, J., Chlorobiphenyls in bound and non-bound lipids of fishes; comparison of different extraction methods. Chemosphere, 1988. 17(9): p. 1803-1810.

57. Booij, K. and C. Van den Berg, Comparison of techniques for the extraction of lipids and PCBs from benthic invertebrates. Bulletin of environmental contamination and toxicology, 1994. 53(1): p. 71-76.

58. Han, X. and R.W. Gross, Shotgun lipidomics: electrospray ionization mass spectrometric analysis and quantitation of cellular lipidomes directly from crude extracts of biological samples. Mass Spectrom Rev, 2005. 24(3): p. 367-412.

59. Cajka, T. and O. Fiehn, Comprehensive analysis of lipids in biological systems by liquid chromatography-mass spectrometry. Trends Analyt Chem, 2014. 61: p. 192-206.

60. Ovcacikova, M., et al., Retention behavior of lipids in reversed-phase ultrahighperformance liquid chromatography-electrospray ionization mass spectrometry. $\mathrm{J}$ Chromatogr A, 2016. 1450: p. 76-85.

61. Lisa, M., E. Cifkova, and M. Holcapek, Lipidomic profiling of biological tissues using offline two-dimensional high-performance liquid chromatography-mass spectrometry. J Chromatogr A, 2011. 1218(31): p. 5146-56.

62. Groessl, M., S. Graf, and R. Knochenmuss, High resolution ion mobility-mass spectrometry for separation and identification of isomeric lipids. Analyst, 2015. 140(20): p. 6904-11.

63. Hines, K.M., et al., Evaluation of Collision Cross Section Calibrants for Structural Analysis of Lipids by Traveling Wave Ion Mobility-Mass Spectrometry. Anal Chem, 2016. 88(14): p. 7329-36.

64. Bowman, A.P., R.R. Abzalimov, and A.A. Shvartsburg, Broad Separation of Isomeric Lipids by High-Resolution Differential Ion Mobility Spectrometry with Tandem Mass Spectrometry. J Am Soc Mass Spectrom, 2017. 28(8): p. 1552-1561.

65. Vasilopoulou, C.G., et al., Trapped ion mobility spectrometry and PASEF enable in-depth lipidomics from minimal sample amounts. Nat Commun, 2020. 11(1): p. 331.

66. Paglia, G. and G. Astarita, Metabolomics and lipidomics using traveling-wave ion mobility mass spectrometry. Nat Protoc, 2017. 12(4): p. 797-813.

67. Zandkarimi, F. and L.M. Brown, Application of Ion Mobility Mass Spectrometry in Lipidomics. Adv Exp Med Biol, 2019. 1140: p. 317-326.

68. Kalo, P., et al., Regiospecific determination of short-chain triacylglycerols in butterfat by normal-phase HPLC with on-line electrospray-tandem mass spectrometry. Lipids, 2004. 39(9): p. 915-28.

69. Fauconnot, L., et al., Quantitative analysis of triacylglycerol regioisomers in fats and oils using reversed-phase high-performance liquid chromatography and atmospheric pressure chemical ionization mass spectrometry. Rapid Commun Mass Spectrom, 2004. 18(2): p. 218-24. 
70. MacDougall, K.M., et al., Triacylglycerol profiling of microalgae strains for biofuel feedstock by liquid chromatography-high-resolution mass spectrometry. Anal Bioanal Chem, 2011. 401(8): p. 2609-16.

71. Ikeda, K., et al., Global analysis of triacylglycerols including oxidized molecular species by reverse-phase high resolution LC/ESI-QTOF MS/MS. J Chromatogr B Analyt Technol Biomed Life Sci, 2009. 877(25): p. 2639-47.

72. Kim, H., et al., Quantitative analysis of phosphatidylcholines and phosphatidylethanolamines in urine of patients with breast cancer by nanoflow liquid chromatography/tandem mass spectrometry. Anal Bioanal Chem, 2009. 393(6-7): $\mathrm{p}$. 1649-56.

73. Schwarz, E., et al., High throughput lipidomic profiling of schizophrenia and bipolar disorder brain tissue reveals alterations of free fatty acids, phosphatidylcholines, and ceramides. J Proteome Res, 2008. 7(10): p. 4266-77.

74. Henderson, C.M., et al., Analysis of major phospholipid species and ergosterol in fermenting industrial yeast strains using atmospheric pressure ionization ion-trap mass spectrometry. J Agric Food Chem, 2011. 59(24): p. 12761-70.

75. Pacetti, D., et al., High-performance liquid chromatography/electrospray ionization iontrap tandem mass spectrometric analysis and quantification of phosphatidylcholine molecular species in the serum of cystic fibrosis subjects supplemented with docosahexaenoic acid. Rapid Commun Mass Spectrom, 2004. 18(20): p. 2395-400.

76. Yin, R., et al., High spatial resolution imaging of biological tissues using nanospray desorption electrospray ionization mass spectrometry. Nat Protoc, 2019. 14(12): p. 34453470.

77. Van Berkel, G.J., et al., Liquid microjunction surface sampling probe electrospray mass spectrometry for detection of drugs and metabolites in thin tissue sections. J Mass Spectrom, 2008. 43(4): p. 500-8.

78. $\quad$ Fowble, K.L., et al., Development of "Laser Ablation Direct Analysis in Real Time Imaging" Mass Spectrometry: Application to Spatial Distribution Mapping of Metabolites Along the Biosynthetic Cascade Leading to Synthesis of Atropine and Scopolamine in Plant Tissue. Anal Chem, 2017. 89(6): p. 3421-3429.

79. Zou, J., et al., Ambient Mass Spectrometry Imaging with Picosecond Infrared Laser Ablation Electrospray Ionization (PIR-LAESI). Anal Chem, 2015. 87(24): p. 12071-9.

80. Niehaus, M., et al., Transmission-mode MALDI-2 mass spectrometry imaging of cells and tissues at subcellular resolution. Nat Methods, 2019. 16(9): p. 925-931.

81. Eikel, D., et al., Liquid extraction surface analysis mass spectrometry (LESA-MS) as a novel profiling tool for drug distribution and metabolism analysis: the terfenadine example. Rapid Commun Mass Spectrom, 2011. 25(23): p. 3587-96.

82. Kraft, M.L. and H.A. Klitzing, Imaging lipids with secondary ion mass spectrometry. Biochim Biophys Acta, 2014. 1841(8): p. 1108-19.

83. Benninghoven, A., F.G. Rudenauer, and H.W. Werner, Secondary ion mass spectrometry: basic concepts, instrumental aspects, applications and trends. 1987, United States: WileyInterscience.

84. Campbell, D.I., et al., Improved spatial resolution in the imaging of biological tissue using desorption electrospray ionization. Anal Bioanal Chem, 2012. 404(2): p. 389-98.

85. Haddad, R., et al., Easy ambient sonic-spray ionization mass spectrometry combined with thin-layer chromatography. Anal Chem, 2008. 80(8): p. 2744-50.

86. Karas, M., D. Bachmann, and F. Hillenkamp, Influence of the wavelength in highirradiance ultraviolet laser desorption mass spectrometry of organic molecules. Analytical Chemistry, 2002. 57(14): p. 2935-2939.

87. Lorenz, M., O.S. Ovchinnikova, and G.J. Van Berkel, Fully automated laser ablation liquid capture surface analysis using nanoelectrospray ionization mass spectrometry. Rapid Commun Mass Spectrom, 2014. 28(11): p. 1312-20. 
88. Luo, Z., et al., Air flow-assisted ionization imaging mass spectrometry method for easy whole-body molecular imaging under ambient conditions. Anal Chem, 2013. 85(5): p. 2977-82.

89. Nemes, P. and A. Vertes, Laser ablation electrospray ionization for atmospheric pressure, in vivo, and imaging mass spectrometry. Anal Chem, 2007. 79(21): p. 8098-106.

90. Otsuka, Y., et al., Scanning probe electrospray ionization for ambient mass spectrometry. Rapid Commun Mass Spectrom, 2012. 26(23): p. 2725-32.

91. Pan, N., et al., The single-probe: a miniaturized multifunctional device for single cell mass spectrometry analysis. Anal Chem, 2014. 86(19): p. 9376-80.

92. Roach, P.J., J. Laskin, and A. Laskin, Nanospray desorption electrospray ionization: an ambient method for liquid-extraction surface sampling in mass spectrometry. Analyst, 2010. 135(9): p. 2233-6.

93. Takats, Z., et al., Mass spectrometry sampling under ambient conditions with desorption electrospray ionization. Science, 2004. 306(5695): p. 471-3.

94. Wachs, T. and J. Henion, Electrospray device for coupling microscale separations and other miniaturized devices with electrospray mass spectrometry. Anal Chem, 2001. 73(3): p. 632-8.

95. Winograd, N., The magic of cluster SIMS. 2005, ACS Publications.

96. Rabbani, S., et al., TOF-SIMS with argon gas cluster ion beams: a comparison with C60+. Analytical chemistry, 2011. 83(10): p. 3793-3800.

97. Sheraz, S., et al., Prospect of increasing secondary ion yields in ToF-SIMS using water cluster primary ion beams. Surface and Interface Analysis, 2014. 46(S1): p. 51-53.

98. Cardiff, R.D., C.H. Miller, and R.J. Munn, Manual hematoxylin and eosin staining of mouse tissue sections. Cold Spring Harb Protoc, 2014. 2014(6): p. 655-8.

99. Ramos-Vara, J.A. and M.A. Miller, When Tissue Antigens and Antibodies Get Along:Revisiting the Technical Aspects of Immunohistochemistry-The Red, Brown, and Blue Technique. Veterinary Pathology, 2014. 51(1): p. 42-87.

100. Mehlem, A., et al., Imaging of neutral lipids by oil red $O$ for analyzing the metabolic status in health and disease. Nature Protocols, 2013. 8(6): p. 1149-1154.

101. Haines, D.M. and B.J. Chelack, Technical Considerations for Developing Enzyme Immunohistochemical Staining Procedures on Formalin-Fixed Paraffin-Embedded Tissues for Diagnostic Pathology. Journal of Veterinary Diagnostic Investigation, 1991. 3(1): p. 101-112.

102. Qi, Z., et al., Reliable Gene Expression Profiling from Small and Hematoxylin and EosinStained Clinical Formalin-Fixed, Paraffin-Embedded Specimens Using the HTG EdgeSeq Platform. The Journal of Molecular Diagnostics, 2019. 21(5): p. 796-807.

103. Fischer, A.H., et al., Cryosectioning tissues. CSH Protoc, 2008. 2008(8).

104. Soltwisch, J., et al., Ion Yields in UV-MALDI Mass Spectrometry As a Function of Excitation Laser Wavelength and Optical and Physico-Chemical Properties of Classical and Halogen-Substituted MALDI Matrixes. Analytical Chemistry, 2012. 84(15): p. 65676576.

105. S., H. and M.-C. S.O., Fundamentals of MALDI-ToF-MS Analysis. 2017: Springer Singapore.

106. Knochenmuss, R., The Coupled Chemical and Physical Dynamics Model of MALDI. Annu Rev Anal Chem (Palo Alto Calif), 2016. 9(1): p. 365-85.

107. Moon, J.H., et al., Formation of gas-phase peptide ions and their dissociation in MALDI: insights from kinetic and ion yield studies. Mass Spectrom Rev, 2015. 34(2): p. 94-115.

108. Knochenmuss, R., Ion formation mechanisms in UV-MALDI. Analyst, 2006. 131(9): p. 966-86.

109. Karas, M. and R. Kruger, Ion formation in MALDI: the cluster ionization mechanism. Chem Rev, 2003. 103(2): p. 427-40.

110. Knochenmuss, R. and R. Zenobi, MALDI ionization: the role of in-plume processes. Chem Rev, 2003. 103(2): p. 441-52. 
111. Dreisewerd, K., The desorption process in MALDI. Chem Rev, 2003. 103(2): p. 395-426.

112. Zenobi, R. and R. Knochenmuss, Ion formation in MALDI mass spectrometry. Mass Spectrometry Reviews, 1998. 17(5): p. 337-366.

113. Beavis, R.C., T. Chaudhary, and B.T. Chait, $\alpha$-Cyano-4-hydroxycinnamic acid as a matrix for matrixassisted laser desorption mass spectromtry. Organic Mass Spectrometry, 1992. 27(2): p. 156-158.

114. Vermillion-Salsbury, R.L. and D.M. Hercules, 9-aminoacridine as a matrix for negative mode matrix-assisted laser desorption/ionization. Rapid Communications in Mass Spectrometry, 2002. 16(16): p. 1575-1581.

115. Yamagaki, T. and H. Nakanishi, A new technique distinguishing alpha2-3 sialyl linkage from alpha2-6 linkage in sialyllactoses and sialyl-N-acetyllactosamines by post-source decay fragmentation method of MALDI-TOF mass spectrometry. Glycoconj J, 1999. 16(8): p. 385-9.

116. Waldchen, F., et al., Multifunctional Reactive MALDI Matrix Enabling High-Lateral Resolution Dual Polarity MS Imaging and Lipid C horizontal lineC Position-Resolved MS(2) Imaging. Anal Chem, 2020. 92(20): p. 14130-14138.

117. Shariatgorji, M., et al., Pyrylium Salts as Reactive Matrices for MALDI-MS Imaging of Biologically Active Primary Amines. J Am Soc Mass Spectrom, 2015. 26(6): p. 934-9.

118. Zhao, X., et al., Combination Strategy of Reactive and Catalytic Matrices for Qualitative and Quantitative Profiling of N-Glycans in MALDI-MS. Anal Chem, 2019. 91(14): p. 9251-9258.

119. Veličković, D., et al., Response Surface Methodology As a New Approach for Finding Optimal MALDI Matrix Spraying Parameters for Mass Spectrometry Imaging. Journal of the American Society for Mass Spectrometry, 2020. 31(3): p. 508-516.

120. Gemperline, E., S. Rawson, and L. Li, Optimization and Comparison of Multiple MALDI Matrix Application Methods for Small Molecule Mass Spectrometric Imaging. Analytical Chemistry, 2014. 86(20): p. 10030-10035.

121. Zavalin, A., et al., Direct imaging of single cells and tissue at sub-cellular spatial resolution using transmission geometry MALDI MS. Journal of Mass Spectrometry, 2012. 47(11): p. 1473-1481.

122. Murphy, R.C., et al., MALDI imaging of lipids after matrix sublimation/deposition. Biochimica et Biophysica Acta (BBA) - Molecular and Cell Biology of Lipids, 2011. 1811(11): p. 970-975.

123. Hankin, J.A., R.M. Barkley, and R.C. Murphy, Sublimation as a method of matrix application for mass spectrometric imaging. Journal of the American Society for Mass Spectrometry, 2007. 18(9): p. 1646-1652.

124. Bouschen, W., et al., Matrix vapor deposition/recrystallization and dedicated spray preparation for high-resolution scanning microprobe matrix-assisted laser desorption/ionization imaging mass spectrometry (SMALDI-MS) of tissue and single cells. Rapid Communications in Mass Spectrometry, 2010. 24(3): p. 355-364.

125. Yang, J. and R.M. Caprioli, Matrix Sublimation/Recrystallization for Imaging Proteins by Mass Spectrometry at High Spatial Resolution. Analytical Chemistry, 2011. 83(14): p. 5728-5734.

126. Lai, Y.-H. and Y.-S. Wang, Matrix-Assisted Laser Desorption/Ionization Mass Spectrometry: Mechanistic Studies and Methods for Improving the Structural Identification of Carbohydrates. Mass spectrometry (Tokyo, Japan), 2017. 6(Spec Iss 2): p. S0072-S0072.

127. Moser, A., K. Range, and D.M. York, Accurate proton affinity and gas-phase basicity values for molecules important in biocatalysis. J Phys Chem B, 2010. 114(43): p. 1391121.

128. Harrison, A.G., The gas-phase basicities and proton affinities of amino acids and peptides. Mass Spectrometry Reviews, 1997. 16(4): p. 201-217. 
129. Schnier, P.D., D.S. Gross, and E.R. Williams, Electrostatic forces and dielectric polarizability of multiply protonated gas-phase cytochrome c ions probed by ion/molecule chemistry. Journal of the American Chemical Society, 1995. 117(25): p. 6747-6757.

130. Jebber, K.A., et al., Ab initio and experimental studies on the protonation of glucose in the gas phase. Journal of the American Chemical Society, 1996. 118(43): p. 10515-10524.

131. Tsai, M.T., et al., Ion-to-neutral ratio of 2, 5-dihydroxybenzoic acid in matrix-assisted laser desorption/ionization. Rapid Communications in Mass Spectrometry, 2013. 27(9): p. 955-963.

132. Bae, Y.J., et al., Degree of ionization in MALDI of peptides: thermal explanation for the gas-phase ion formation. Journal of the American Society for Mass Spectrometry, 2012. 23(8): p. 1326-1335.

133. Shimma, S., et al., Alternative two-step matrix application method for imaging mass spectrometry to avoid tissue shrinkage and improve ionization efficiency. Journal of Mass Spectrometry, 2013. 48(12): p. 1285-1290.

134. Sugiura, Y., S. Shimma, and M. Setou, Two-Step Matrix Application Technique To Improve Ionization Efficiency for Matrix-Assisted Laser Desorption/Ionization in Imaging Mass Spectrometry. Analytical Chemistry, 2006. 78(24): p. 8227-8235.

135. Shanta, S.R., et al., A new combination MALDI matrix for small molecule analysis: application to imaging mass spectrometry for drugs and metabolites. Analyst, 2012. 137(24): p. 5757-62.

136. Meier, M.A.R., N. Adams, and U.S. Schubert, Statistical Approach To Understand MALDI-TOFMS Matrices: Discovery and Evaluation of New MALDI Matrices.

Analytical Chemistry, 2007. 79(3): p. 863-869.

137. Leszyk, J.D., Evaluation of the new MALDI matrix 4-chloro-alpha-cyanocinnamic acid. J Biomol Tech, 2010. 21(2): p. 81-91.

138. Dong, X., et al., Graphene as a Novel Matrix for the Analysis of Small Molecules by MALDI-TOF MS. Analytical Chemistry, 2010. 82(14): p. 6208-6214.

139. Flinders, B., et al., The use of hydrazine-based derivatization reagents for improved sensitivity and detection of carbonyl containing compounds using MALDI-MSI. Analytical and Bioanalytical Chemistry, 2015. 407(8): p. 2085-2094.

140. Guo, S., et al., Enhancement of On-tissue Chemical Derivatization by Laser-Assisted Tissue Transfer for MALDI MS Imaging. Analytical Chemistry, 2020. 92(1): p. 14311438.

141. Nishikaze, T., Sensitive and Structure-Informative $<i>N</ i>$-Glycosylation Analysis by MALDI-MS; Ionization, Fragmentation, and Derivatization. Mass Spectrometry, 2017. 6(1): p. A0060-A0060.

142. Zhou, Q., A. Fülöp, and C. Hopf, Recent developments of novel matrices and on-tissue chemical derivatization reagents for MALDI-MSI. Analytical and Bioanalytical Chemistry, 2021. 413(10): p. 2599-2617.

143. Zhang, Y., et al., Sensitive and robust MALDI-TOF-MS glycomics analysis enabled by Girard's reagent T on-target derivatization (GTOD) of reducing glycans. Analytica Chimica Acta, 2019. 1048: p. 105-114.

144. Soltwisch, J., et al., Mass spectrometry imaging with laser-induced postionization. Science, 2015. 348(6231): p. 211-215.

145. Gross, J.H., Mass spectrometry: a textbook. 2006: Springer Science \& Business Media.

146. Kanawati, B. and K.P. Wanczek, Characterization of a new open cylindrical ion cyclotron resonance cell with unusual geometry. Rev Sci Instrum, 2007. 78(7): p. 074102.

147. Douglas, D.J., A.J. Frank, and D. Mao, Linear ion traps in mass spectrometry. Mass Spectrometry Reviews, 2005. 24(1): p. 1-29.

148. Kornienko, O., et al., Micro ion trap mass spectrometry. Rapid Communications in Mass Spectrometry, 1999. 13(1): p. 50-53. 
149. Ouyang, Z., E.R. Badman, and R.G. Cooks, Characterization of a serial array of miniature cylindrical ion trap mass analyzers. Rapid Communications in Mass Spectrometry, 1999. 13(24): p. 2444-2449.

150. Patterson, G.E., et al., Miniature Cylindrical Ion Trap Mass Spectrometer. Analytical Chemistry, 2002. 74(24): p. 6145-6153.

151. Singh, R., V. Jayaram, and P.T.A. Reilly, Duty cycle-based isolation in linear quadrupole ion traps. International Journal of Mass Spectrometry, 2013. 343-344: p. 45-49.

152. Schwartz, J.C., J.E.P. Syka, and I. Jardine, High resolution on a quadrupole ion trap mass spectrometer. Journal of the American Society for Mass Spectrometry, 1991. 2(3): p. 198204.

153. Spraggins, J.M. and R.M. Caprioli, High-speed MALDI-TOF imaging mass spectrometry: rapid ion image acquisition and considerations for next generation instrumentation. Journal of the American Society for mass Spectrometry, 2011. 22(6): p. 1022-1031.

154. Marshall, A.G., C.L. Hendrickson, and G.S. Jackson, Fourier transform ion cyclotron resonance mass spectrometry: A primer. Mass Spectrometry Reviews, 1998. 17(1): p. 135.

155. Makarov, A., Electrostatic Axially Harmonic Orbital Trapping: A High-Performance Technique of Mass Analysis. Analytical Chemistry, 2000. 72(6): p. 1156-1162.

156. Blakney, G.T., C.L. Hendrickson, and A.G. Marshall, Predator data station: A fast data acquisition system for advanced FT-ICR MS experiments. International Journal of Mass Spectrometry, 2011.306(2): p. 246-252.

157. Denisov, E., et al., Orbitrap mass spectrometry with resolving powers above 1,000,000. International Journal of Mass Spectrometry, 2012. 325-327: p. 80-85.

158. Sleno, L. and D.A. Volmer, Ion activation methods for tandem mass spectrometry. Journal of Mass Spectrometry, 2004. 39(10): p. 1091-1112.

159. Schuhmann, K., et al., Bottom-up shotgun lipidomics by higher energy collisional dissociation on LTQ Orbitrap mass spectrometers. Analytical chemistry, 2011. 83(14): p. $5480-5487$.

160. Thomas, M.C., et al., Ozone-induced dissociation: elucidation of double bond position within mass-selected lipid ions. Anal Chem, 2008. 80(1): p. 303-11.

161. Williams, P.E., et al., Pinpointing Double Bond and sn-Positions in Glycerophospholipids via Hybrid $193 \mathrm{~nm}$ Ultraviolet Photodissociation (UVPD) Mass Spectrometry. J Am Chem Soc, 2017. 139(44): p. 15681-15690.

162. Randolph, C.E., S.J. Blanksby, and S.A. McLuckey, Toward Complete Structure Elucidation of Glycerophospholipids in the Gas Phase through Charge Inversion Ion/Ion Chemistry. Anal Chem, 2020.92(1): p. 1219-1227.

163. Zhao, Y., et al., Identification and Quantitation of $C=C$ Location Isomers of Unsaturated Fatty Acids by Epoxidation Reaction and Tandem Mass Spectrometry. Anal Chem, 2017. 89(19): p. 10270-10278.

164. Cao, W., et al., Large-scale lipid analysis with $C=C$ location and sn-position isomer resolving power. Nat Commun, 2020. 11(1): p. 375.

165. Pham, H.T., et al., Structural characterization of glycerophospholipids by combinations of ozone- and collision-induced dissociation mass spectrometry: the next step towards "topdown" lipidomics. Analyst, 2014. 139(1): p. 204-14.

166. Epand, R.M., et al., Substrate Chirality and Specificity of Diacylglycerol Kinases and the Multisubstrate Lipid Kinase. Biochemistry, 2007. 46(49): p. 14225-14231.

167. Poad, B.L.J., et al., Ozone-induced dissociation on a modified tandem linear ion-trap: Observations of different reactivity for isomeric lipids. Journal of the American Society for Mass Spectrometry, 2010. 21(12): p. 1989-1999.

168. Tang, L., Multiomics sequencing goes spatial. Nat Methods, 2021. 18(1): p. 31.

169. Wenk, M.R., The emerging field of lipidomics. Nat Rev Drug Discov, 2005. 4(7): p. 594610. 
170. Blanksby, S.J. and T.W. Mitchell, Advances in mass spectrometry for lipidomics. Annu Rev Anal Chem (Palo Alto Calif), 2010. 3(1): p. 433-65.

171. Shevchenko, A. and K. Simons, Lipidomics: coming to grips with lipid diversity. Nat Rev Mol Cell Biol, 2010. 11(8): p. 593-8.

172. McDonnell, L.A. and R.M. Heeren, Imaging mass spectrometry. Mass Spectrom Rev, 2007. 26(4): p. 606-43.

173. Berry, K.A., et al., MALDI imaging of lipid biochemistry in tissues by mass spectrometry. Chem Rev, 2011. 111(10): p. 6491-512.

174. Passarelli, M.K. and N. Winograd, Lipid imaging with time-of-flight secondary ion mass spectrometry (ToF-SIMS). Biochim Biophys Acta, 2011. 1811(11): p. 976-90.

175. Eberlin, L.S., et al., Desorption electrospray ionization mass spectrometry for lipid characterization and biological tissue imaging. Biochim Biophys Acta, 2011. 1811(11): p. 946-60.

176. Goodwin, R.J.A., Sample preparation for mass spectrometry imaging: small mistakes can lead to big consequences. J Proteomics, 2012. 75(16): p. 4893-4911.

177. Sugimoto, M., et al., Imaging Mass Spectrometry Reveals Acyl-Chain- and RegionSpecific Sphingolipid Metabolism in the Kidneys of Sphingomyelin Synthase 2-Deficient Mice. PLoS One, 2016. 11(3): p. e0152191.

178. Eberlin, L.S., et al., Classifying human brain tumors by lipid imaging with mass spectrometry. Cancer Res, 2012. 72(3): p. 645-54.

179. Hall, Z., et al., Lipid zonation and phospholipid remodeling in nonalcoholic fatty liver disease. Hepatology, 2017. 65(4): p. 1165-1180.

180. Balog, J., et al., Intraoperative tissue identification using rapid evaporative ionization mass spectrometry. Sci Transl Med, 2013. 5(194): p. 194ra93.

181. Eberlin, L.S., et al., Ambient mass spectrometry for the intraoperative molecular diagnosis of human brain tumors. Proc Natl Acad Sci U S A, 2013. 110(5): p. 1611-6.

182. Angel, P.M., et al., Enhanced sensitivity for high spatial resolution lipid analysis by negative ion mode matrix assisted laser desorption ionization imaging mass spectrometry. Anal Chem, 2012. 84(3): p. 1557-64.

183. Norris, J.L. and R.M. Caprioli, Analysis of tissue specimens by matrix-assisted laser desorption/ionization imaging mass spectrometry in biological and clinical research. Chem Rev, 2013. 113(4): p. 2309-42.

184. Kompauer, M., S. Heiles, and B. Spengler, Atmospheric pressure MALDI mass spectrometry imaging of tissues and cells at 1.4-mum lateral resolution. Nat Methods, 2017. 14(1): p. 90-96.

185. Ostrowski, S.G., et al., Mass spectrometric imaging of highly curved membranes during Tetrahymena mating. Science, 2004. 305(5680): p. 71-3.

186. Tian, H., et al., Subcellular Chemical Imaging of Antibiotics in Single Bacteria Using C60-Secondary Ion Mass Spectrometry. Anal Chem, 2017. 89(9): p. 5050-5057.

187. Angerer, T.B., et al., Lipid Heterogeneity Resulting from Fatty Acid Processing in the Human Breast Cancer Microenvironment Identified by GCIB-ToF-SIMS Imaging. Anal Chem, 2016. 88(23): p. 11946-11954.

188. Fletcher, J.S., et al., A new dynamic in mass spectral imaging of single biological cells. Anal Chem, 2008. 80(23): p. 9058-64.

189. Fisher, G.L., et al., A New Method and Mass Spectrometer Design for TOF-SIMS Parallel Imaging $M S / M S$. Anal Chem, 2016. 88(12): p. 6433-40.

190. Passarelli, M.K., et al., The 3D OrbiSIMS-label-free metabolic imaging with subcellular lateral resolution and high mass-resolving power. Nat Methods, 2017. 14(12): p. 11751183.

191. Wu, C., et al., Mass spectrometry imaging under ambient conditions. Mass Spectrom Rev, 2013. 32(3): p. 218-43.

192. Carson, R.H., et al., Imaging regiospecific lipid turnover in mouse brain with desorption electrospray ionization mass spectrometry. J Lipid Res, 2017. 58(9): p. 1884-1892. 
193. Wu, C., et al., Rapid, direct analysis of cholesterol by charge labeling in reactive desorption electrospray ionization. Anal Chem, 2009. 81(18): p. 7618-24.

194. Guo, S., et al., Significantly increased monounsaturated lipids relative to polyunsaturated lipids in six types of cancer microenvironment are observed by mass spectrometry imaging. Sci Rep, 2014. 4: p. 5959.

195. Paton, C.M. and J.M. Ntambi, Biochemical and physiological function of stearoyl-CoA desaturase. Am J Physiol Endocrinol Metab, 2009. 297(1): p. E28-37.

196. Ide, Y., et al., Human breast cancer tissues contain abundant phosphatidylcholine(36ratiol) with high stearoyl-CoA desaturase-1 expression. PLoS One, 2013. 8(4): p. e61204.

197. Eberlin, L.S., et al., Alteration of the lipid profile in lymphomas induced by MYC overexpression. Proc Natl Acad Sci U S A, 2014. 111(29): p. 10450-5.

198. Liu, Y., et al., Elevation of sulfatides in ovarian cancer: an integrated transcriptomic and lipidomic analysis including tissue-imaging mass spectrometry. Mol Cancer, 2010. 9(1): p. 186.

199. Scott, A.J., et al., Host-based lipid inflammation drives pathogenesis in Francisella infection. Proc Natl Acad Sci U S A, 2017. 114(47): p. 12596-12601.

200. Hiraide, T., et al., Accumulation of arachidonic acid-containing phosphatidylinositol at the outer edge of colorectal cancer. Sci Rep, 2016. 6: p. 29935.

201. Bestard-Escalas, J., et al., Lipid fingerprint image accurately conveys human colon cell pathophysiologic state: A solid candidate as biomarker. Biochim Biophys Acta, 2016. 1861(12 Pt A): p. 1942-1950.

202. Banno, T., et al., Arachidonic acid containing phosphatidylcholine increases due to microglial activation in ipsilateral spinal dorsal horn following spared sciatic nerve injury. PLoS One, 2017. 12(5): p. e0177595.

203. Yang, H.J., et al., Axonal gradient of arachidonic acid-containing phosphatidylcholine and its dependence on actin dynamics. J Biol Chem, 2012. 287(8): p. 5290-300.

204. Seyer, A., et al., Lipidomic and spatio-temporal imaging of fat by mass spectrometry in mice duodenum during lipid digestion. PLoS One, 2013. 8(4): p. e58224.

205. Horn, P.J., et al., Imaging heterogeneity of membrane and storage lipids in transgenic Camelina sativa seeds with altered fatty acid profiles. Plant J, 2013. 76(1): p. 138-50.

206. Zacek, P., et al., Quantitation of isobaric phosphatidylcholine species in human plasma using a hybrid quadrupole linear ion-trap mass spectrometer. J Lipid Res, 2016. 57(12): p. 2225-2234.

207. Park, E.S., et al., Phosphatidylcholine alteration identified using MALDI imaging MS in $H B V$-infected mouse livers and virus-mediated regeneration defects. PLoS One, 2014. 9(8): p. e103955.

208. Postle, A.D. and A.N. Hunt, Dynamic lipidomics with stable isotope labelling. J Chromatogr B Analyt Technol Biomed Life Sci, 2009. 877(26): p. 2716-21.

209. Louie, K.B., et al., Mass spectrometry imaging for in situ kinetic histochemistry. Sci Rep, 2013. 3: p. 1656.

210. Sugiyama, E., I. Yao, and M. Setou, Visualization of local phosphatidylcholine synthesis within hippocampal neurons using a compartmentalized culture system and imaging mass spectrometry. Biochem Biophys Res Commun, 2018. 495(1): p. 1048-1054.

211. Brandsma, J., et al., Stable isotope analysis of dynamic lipidomics. Biochim Biophys Acta Mol Cell Biol Lipids, 2017. 1862(8): p. 792-796.

212. Petkovic, M., et al., Detection of individual phospholipids in lipid mixtures by matrixassisted laser desorption/ionization time-of-flight mass spectrometry: Phosphatidylcholine prevents the detection of further species. Analytical Biochemistry, 2001. 289(2): p. 202216.

213. Soltwisch, J., et al., Mass spectrometry imaging with laser-induced postionization. Science, 2015. 348(6231): p. 211-5. 
214. Ellis, S.R., et al., Laser post-ionisation combined with a high resolving power orbitrap mass spectrometer for enhanced MALDI-MS imaging of lipids. Chem Commun (Camb), 2017. 53(53): p. 7246-7249.

215. Ellis, S.R., et al., More from less: high-throughput dual polarity lipid imaging of biological tissues. Analyst, 2016. 141(12): p. 3832-41.

216. Kaya, I., et al., Novel Trimodal MALDI Imaging Mass Spectrometry (IMS3) at 10 mum Reveals Spatial Lipid and Peptide Correlates Implicated in Abeta Plaque Pathology in Alzheimer's Disease. ACS Chem Neurosci, 2017. 8(12): p. 2778-2790.

217. Ryan, E. and G.E. Reid, Chemical Derivatization and Ultrahigh Resolution and Accurate Mass Spectrometry Strategies for "Shotgun" Lipidome Analysis. Acc Chem Res, 2016. 49(9): p. 1596-604.

218. Guillou, H., et al., The key roles of elongases and desaturases in mammalian fatty acid metabolism: Insights from transgenic mice. Prog Lipid Res, 2010. 49(2): p. 186-99.

219. Ryan, E., et al., Detailed Structural Characterization of Sphingolipids via $193 \mathrm{~nm}$ Ultraviolet Photodissociation and Ultra High Resolution Tandem Mass Spectrometry. J Am Soc Mass Spectrom, 2017. 28(7): p. 1406-1419.

220. Ma, X. and Y. Xia, Pinpointing double bonds in lipids by Paterno-Buchi reactions and mass spectrometry. Angew Chem Int Ed Engl, 2014. 53(10): p. 2592-6.

221. Kanu, A.B., et al., Ion mobility-mass spectrometry. J Mass Spectrom, 2008. 43(1): p. 1-22.

222. Vaysse, P.M., et al., Mass spectrometry imaging for clinical research - latest developments, applications, and current limitations. Analyst, 2017. 142(15): p. 26902712.

223. Lazar, A.N., et al., Time-of-flight secondary ion mass spectrometry (TOF-SIMS) imaging reveals cholesterol overload in the cerebral cortex of Alzheimer disease patients. Acta Neuropathol, 2013. 125(1): p. 133-44.

224. Chaurand, P., S.A. Schwartz, and R.M. Caprioli, Assessing protein patterns in disease using imaging mass spectrometry. J Proteome Res, 2004. 3(2): p. 245-52.

225. Chen, Y., et al., Imaging MALDI mass spectrometry using an oscillating capillary nebulizer matrix coating system and its application to analysis of lipids in brain from a mouse model of Tay-Sachs/Sandhoff disease. Anal Chem, 2008. 80(8): p. 2780-8.

226. Hoefler, B.C., et al., Enzymatic resistance to the lipopeptide surfactin as identified through imaging mass spectrometry of bacterial competition. Proc Natl Acad Sci U S A, 2012. 109(32): p. 13082-7.

227. Schulz, S., et al., Advanced MALDI mass spectrometry imaging in pharmaceutical research and drug development. Curr Opin Biotechnol, 2019. 55: p. 51-59.

228. Castellino, S., M.R. Groseclose, and D. Wagner, MALDI imaging mass spectrometry: bridging biology and chemistry in drug development. Bioanalysis, 2011. 3(21): p. 242741.

229. Ellis, S.R., A.L. Bruinen, and R.M. Heeren, A critical evaluation of the current state-ofthe-art in quantitative imaging mass spectrometry. Anal Bioanal Chem, 2014. 406(5): p. 1275-89.

230. Taban, I.M., et al., Imaging of peptides in the rat brain using MALDI-FTICR mass spectrometry. J Am Soc Mass Spectrom, 2007. 18(1): p. 145-51.

231. Bowman, A.P., R.M.A. Heeren, and S.R. Ellis, Advances in mass spectrometry imaging enabling observation of localised lipid biochemistry within tissues. TrAC Trends in Analytical Chemistry, 2018.

232. Zhao, C., et al., Identification of glycerophospholipid fatty acid remodeling by using mass spectrometry imaging in bisphenol $S$ induced mouse liver. Chinese Chemical Letters, 2018. 29(8): p. 1281-1283.

233. Sans, M., C.L. Feider, and L.S. Eberlin, Advances in mass spectrometry imaging coupled to ion mobility spectrometry for enhanced imaging of biological tissues. Curr Opin Chem Biol, 2018. 42: p. 138-146. 
234. Bielow, C., et al., On Mass Ambiguities in High-Resolution Shotgun Lipidomics. Anal Chem, 2017. 89(5): p. 2986-2994.

235. Shaw, J.B., et al., 21 Tesla Fourier Transform Ion Cyclotron Resonance Mass Spectrometer Greatly Expands Mass Spectrometry Toolbox. J Am Soc Mass Spectrom, 2016. 27(12): p. 1929-1936.

236. Kooijman, P.C., et al., Increased throughput and ultra-high mass resolution in DESI FTICR MS imaging through new-generation external data acquisition system and advanced data processing approaches. Sci Rep, 2019. 9(1): p. 8.

237. Smith, D.F., et al., Absorption mode FTICR mass spectrometry imaging. Anal Chem, 2013. 85(23): p. 11180-4.

238. Qi, Y., et al., Absorption-mode: the next generation of Fourier transform mass spectra. Anal Chem, 2012. 84(6): p. 2923-9.

239. Stopka, S.A., et al., Ambient Metabolic Profiling and Imaging of Biological Samples with Ultrahigh Molecular Resolution Using Laser Ablation Electrospray Ionization 21 Tesla FTICR Mass Spectrometry. Anal Chem, 2019. 91(8): p. 5028-5035.

240. Schwudke, D., et al., Shotgun lipidomics on high resolution mass spectrometers. Cold Spring Harb Perspect Biol, 2011. 3(9): p. a004614.

241. Hu, C., Q. Duan, and X. Han, Strategies to Improve/Eliminate the Limitations in Shotgun Lipidomics. Proteomics, 2020. 20(11): p. e1900070.

242. Wildburger, N.C., et al., ESI-MS/MS and MALDI-IMS Localization Reveal Alterations in Phosphatidic Acid, Diacylglycerol, and DHA in Glioma Stem Cell Xenografts. J Proteome Res, 2015. 14(6): p. 2511-9.

243. Holcapek, M., et al., Lipidomic analysis of plasma, erythrocytes and lipoprotein fractions of cardiovascular disease patients using UHPLC/MS, MALDI-MS and multivariate data analysis. J Chromatogr B Analyt Technol Biomed Life Sci, 2015. 990: p. 52-63.

244. Korte, A.R., et al., Subcellular-level resolution MALDI-MS imaging of maize leaf metabolites by MALDI-linear ion trap-Orbitrap mass spectrometer. Anal Bioanal Chem, 2015. 407(8): p. 2301-9.

245. Olsen, J.V., et al., Parts per million mass accuracy on an Orbitrap mass spectrometer via lock mass injection into a C-trap. Mol Cell Proteomics, 2005. 4(12): p. 2010-21.

246. Makarov, A., et al., Dynamic range of mass accuracy in LTQ Orbitrap hybrid mass spectrometer. J Am Soc Mass Spectrom, 2006. 17(7): p. 977-982.

247. Scigelova, M. and A. Makarov, Orbitrap mass analyzer--overview and applications in proteomics. Proteomics, 2006. 6 Suppl 2: p. 16-21.

248. Marshall, A.G., C.L. Hendrickson, and G.S. Jackson, Fourier transform ion cyclotron resonance mass spectrometry: a primer. Mass Spectrom Rev, 1998. 17(1): p. 1-35.

249. Nikolaev, E.N., et al., Ion-Cyclotron Resonance Signal-Detection at Multiples of the Cyclotron Frequency. Rapid Communications in Mass Spectrometry, 1990. 4(5): p. 144146.

250. Walker, L.R., et al., Unambiguous identification and discovery of bacterial siderophores by direct injection 21 Tesla Fourier transform ion cyclotron resonance mass spectrometry. Metallomics, 2017. 9(1): p. 82-92.

251. Zimmerman, T.A., et al., Chapter 13: Imaging of cells and tissues with mass spectrometry: adding chemical information to imaging. Methods Cell Biol, 2008. 89: $\mathrm{p}$. 361-90.

252. Hendrickson, C.L., et al., 21 Tesla Fourier Transform Ion Cyclotron Resonance Mass Spectrometer: A National Resource for Ultrahigh Resolution Mass Analysis. J Am Soc Mass Spectrom, 2015. 26(9): p. 1626-32.

253. Smith, D.F., et al., 21 Tesla FT-ICR Mass Spectrometer for Ultrahigh-Resolution Analysis of Complex Organic Mixtures. Anal Chem, 2018. 90(3): p. 2041-2047.

254. Burla, B., et al., MS-based lipidomics of human blood plasma: a community-initiated position paper to develop accepted guidelines. J Lipid Res, 2018. 59(10): p. 2001-2017. 
255. Xiang, X.Z., P.B. Grosshans, and A.G. Marshall, Image Charge-Induced Ion-Cyclotron Orbital Frequency-Shift for Orthorhombic and Cylindrical Ft-Icr Ion Traps. International Journal of Mass Spectrometry and Ion Processes, 1993. 125(1): p. 33-43.

256. Wong, R.L. and I.J. Amster, Experimental Evidence for Space-Charge Effects between Ions of the Same Mass-to-Charge in Fourier-Transform Ion Cyclotron Resonance Mass Spectrometry. Int J Mass Spectrom, 2007. 265(2-3): p. 99-105.

257. Savory, J.J., et al., Parts-per-billion Fourier transform ion cyclotron resonance mass measurement accuracy with a "walking" calibration equation. Anal Chem, 2011. 83(5): p. 1732-6.

258. Schuhmann, K., et al., Shotgun lipidomics on a LTQ Orbitrap mass spectrometer by successive switching between acquisition polarity modes. J Mass Spectrom, 2012. 47(1): p. $96-104$.

259. Almeida, R., et al., Comprehensive lipidome analysis by shotgun lipidomics on a hybrid quadrupole-orbitrap-linear ion trap mass spectrometer. J Am Soc Mass Spectrom, 2015. 26(1): p. 133-48.

260. Simons, B., et al., Shotgun Lipidomics by Sequential Precursor Ion Fragmentation on a Hybrid Quadrupole Time-of-Flight Mass Spectrometer. Metabolites, 2012. 2(1): p. 195213.

261. Velickovic, D., et al., Multimodal MSI in Conjunction with Broad Coverage Spatially Resolved MS(2) Increases Confidence in Both Molecular Identification and Localization. Anal Chem, 2018. 90(1): p. 702-707.

262. Spraggins, J.M., et al., MALDI FTICR IMS of Intact Proteins: Using Mass Accuracy to Link Protein Images with Proteomics Data. J Am Soc Mass Spectrom, 2015. 26(6): p. 974-85.

263. Hendrickson, C.L., et al. Optimized cell geometry for Fourier transform ion cyclotron resonance mass spectrometry. in Proceedings of the 57th ASMS Conference on Mass Spectrometry and Allied Topics. 2009. Philadelphia, PA.

264. Chen, T., et al., Note: Optimized circuit for excitation and detection with one pair of electrodes for improved Fourier transform ion cyclotron resonance mass spectrometry. Rev Sci Instrum, 2014. 85(6): p. 066107.

265. Belov, M.E., et al., Design and Performance of a Novel Interface for Combined MatrixAssisted Laser Desorption Ionization at Elevated Pressure and Electrospray Ionization with Orbitrap Mass Spectrometry. Anal Chem, 2017. 89(14): p. 7493-7501.

266. Blakney, G.T., C.L. Hendrickson, and A.G. Marshall, Predator data station: A fast data acquisition system for advanced FT-ICR MS experiments. International Journal of Mass Spectrometry, 2011.306(2-3): p. 246-252.

267. Xian, F., et al., Automated broadband phase correction of Fourier transform ion cyclotron resonance mass spectra. Anal Chem, 2010. 82(21): p. 8807-12.

268. Chambers, M.C., et al., A cross-platform toolkit for mass spectrometry and proteomics. Nat Biotechnol, 2012. 30(10): p. 918-20.

269. Race, A.M., I.B. Styles, and J. Bunch, Inclusive sharing of mass spectrometry imaging data requires a converter for all. J Proteomics, 2012. 75(16): p. 5111-5112.

270. Husen, P., et al., Analysis of lipid experiments (ALEX): a software framework for analysis of high-resolution shotgun lipidomics data. PLoS One, 2013. 8(11): p. e79736.

271. Spraggins, J.M., et al., High-Performance Molecular Imaging with MALDI Trapped IonMobility Time-of-Flight (timsTOF) Mass Spectrometry. Anal Chem, 2019. 91(22): p. 14552-14560.

272. Campos, A.M., et al., Lipidomics of Mesenchymal Stromal Cells: Understanding the Adaptation of Phospholipid Profile in Response to Pro-Inflammatory Cytokines. J Cell Physiol, 2016. 231(5): p. 1024-32.

273. Harbige, L.S. and M.K. Sharief, Polyunsaturated fatty acids in the pathogenesis and treatment of multiple sclerosis. Br J Nutr, 2007. 98 Suppl 1: p. S46-53. 
274. Vance, J.E. and G. Tasseva, Formation and function of phosphatidylserine and phosphatidylethanolamine in mammalian cells. Biochim Biophys Acta, 2013. 1831(3): p. 543-54.

275. Kim, W.S., C.S. Weickert, and B. Garner, Role of ATP-binding cassette transporters in brain lipid transport and neurological disease. J Neurochem, 2008. 104(5): p. 1145-66.

276. Smith, D.F., et al., Advanced mass calibration and visualization for FT-ICR mass spectrometry imaging. J Am Soc Mass Spectrom, 2012. 23(11): p. 1865-72.

277. Estrada, R. and M.C. Yappert, Alternative approaches for the detection of various phospholipid classes by matrix-assisted laser desorption/ionization time-of-flight mass spectrometry. J Mass Spectrom, 2004. 39(4): p. 412-22.

278. Fuchs, B., et al., A direct and simple method of coupling matrix-assisted laser desorption and ionization time-of-flight mass spectrometry (MALDI-TOF MS) to thin-layer chromatography (TLC) for the analysis of phospholipids from egg yolk. Anal Bioanal Chem, 2007. 389(3): p. 827-34.

279. Bowman, A.P., R.M.A. Heeren, and S.R. Ellis, Advances in mass spectrometry imaging enabling observation of localised lipid biochemistry within tissues. TrAC Trends in Analytical Chemistry, 2018: p. in press, DOI: https://doi.org/10.1016/j.trac.2018.07.012.

280. Gode, D. and D.A. Volmer, Lipid imaging by mass spectrometry - a review. Analyst, 2013. 138(5): p. 1289-315.

281. Murphy, R.C., J.A. Hankin, and R.M. Barkley, Imaging of lipid species by MALDI mass spectrometry. J Lipid Res, 2009. 50 Suppl: p. S317-22.

282. Guo, S., et al., Significantly increased monounsaturated lipids relative to polyunsaturated lipids in six types of cancer microenvironment are observed by mass spectrometry imaging. Scientific Reports, 2014. 4: p. 5959.

283. Paine, M.R.L., et al., Three-Dimensional Mass Spectrometry Imaging Identifies Lipid Markers of Medulloblastoma Metastasis. Sci Rep, 2019. 9(1): p. 2205.

284. Wang, X., et al., Metabolomic profiling of prostate cancer by matrix assisted laser desorption/ionization-Fourier transform ion cyclotron resonance mass spectrometry imaging using Matrix Coating Assisted by an Electric Field (MCAEF). Biochim Biophys Acta Proteins Proteom, 2017. 1865(7): p. 755-767.

285. Scott, A.J., et al., Host-based lipid inflammation drives pathogenesis in Francisella infection. Proceedings of the National Academy of Sciences, 2017. 114(47): p. 1259612601.

286. Blanc, L., et al., Visualization of Mycobacterial Biomarkers and Tuberculosis Drugs in Infected Tissue by MALDI-MS Imaging. Anal Chem, 2018. 90(10): p. 6275-6282.

287. Scupakova, K., et al., Spatial Systems Lipidomics Reveals Nonalcoholic Fatty Liver Disease Heterogeneity. Anal Chem, 2018. 90(8): p. 5130-5138.

288. Hall, Z., et al., Lipid zonation and phospholipid remodeling in nonalcoholic fatty liver disease. Hepatology (Baltimore, Md.), 2017. 65(4): p. 1165-1180.

289. Kettling, H., et al., MALDI mass spectrometry imaging of bioactive lipids in mouse brain with a Synapt G2-S mass spectrometer operated at elevated pressure: improving the analytical sensitivity and the lateral resolution to ten micrometers. Anal Chem, 2014. 86(15): p. 7798-805.

290. Kompauer, M., S. Heiles, and B. Spengler, Atmospheric pressure MALDI mass spectrometry imaging of tissues and cells at 1.4-mm lateral resolution. Nat Meth, 2017. 14(1): p. 90-96.

291. Feenstra, A.D., M.E. Duenas, and Y.J. Lee, Five Micron High Resolution MALDI Mass Spectrometry Imaging with Simple, Interchangeable, Multi-Resolution Optical System. J Am Soc Mass Spectrom, 2017. 28(3): p. 434-442.

292. Korte, A.R., et al., Subcellular-level resolution MALDI-MS imaging of maize leaf metabolites by MALDI-linear ion trap-Orbitrap mass spectrometer. Analytical and Bioanalytical Chemistry, 2015. 407(8): p. 2301-2309. 
293. Zavalin, A., et al., Direct imaging of single cells and tissue at sub-cellular spatial resolution using transmission geometry MALDI MS. J Mass Spectrom, 2012. 47(11): p. i.

294. Kompauer, M., S. Heiles, and B. Spengler, Autofocusing MALDI mass spectrometry imaging of tissue sections and 3D chemical topography of nonflat surfaces. Nat Methods, 2017. 14(12): p. 1156-1158.

295. Jurchen, J.C., S.S. Rubakhin, and J.V. Sweedler, MALDI-MS imaging of features smaller than the size of the laser beam. J Am Soc Mass Spectrom, 2005. 16(10): p. 1654-9.

296. Snel, M.F. and M. Fuller, High-spatial resolution matrix-assisted laser desorption ionization imaging analysis of glucosylceramide in spleen sections from a mouse model of Gaucher disease. Anal Chem, 2010. 82(9): p. 3664-70.

297. Sparvero, L.J., et al., Mapping of phospholipids by MALDI imaging (MALDI-MSI): realities and expectations. Chem Phys Lipids, 2012. 165(5): p. 545-62.

298. Garate, J., et al., Imaging mass spectrometry increased resolution using 2mercaptobenzothiazole and 2,5-diaminonaphtalene matrices: application to lipid distribution in human colon. Anal Bioanal Chem, 2015. 407(16): p. 4697-708.

299. Trim, P.J., et al., Introduction of a $20 \mathrm{kHz} N d$ :YVO4 laser into a hybrid quadrupole timeof-flight mass spectrometer for MALDI-MS imaging. Anal Bioanal Chem, 2010. 397(8): p. 3409-19.

300. Spraggins, J.M. and R.M. Caprioli, High-speed MALDI-TOF imaging mass spectrometry: rapid ion image acquisition and considerations for next generation instrumentation. $\mathrm{J}$ Am Soc Mass Spectrom, 2011. 22(6): p. 1022-31.

301. Nazari, M. and D.C. Muddiman, Cellular-level mass spectrometry imaging using infrared matrix-assisted laser desorption electrospray ionization (IR-MALDESI) by oversampling. Anal Bioanal Chem, 2015. 407(8): p. 2265-71.

302. Wiegelmann, M., K. Dreisewerd, and J. Soltwisch, Influence of the Laser Spot Size, Focal Beam Profile, and Tissue Type on the Lipid Signals Obtained by MALDI-MS Imaging in Oversampling Mode. J Am Soc Mass Spectrom, 2016. 27(12): p. 1952-1964.

303. Barre, F.P.Y., et al., Enhanced Sensitivity Using MALDI Imaging Coupled with Laser Postionization (MALDI-2) for Pharmaceutical Research. Anal Chem, 2019. 91(16): p. 10840-10848.

304. Flinders, B., et al., Cross-Species Molecular Imaging of Bile Salts and Lipids in Liver: Identification of Molecular Structural Markers in Health and Disease. Anal Chem, 2018. 90(20): p. 11835-11846.

305. Hankin, J.A., R.M. Barkley, and R.C. Murphy, Sublimation as a method of matrix application for mass spectrometric imaging. J Am Soc Mass Spectrom, 2007. 18(9): p. 1646-52.

306. Bogie, J.F., et al., Scavenger receptor collectin placenta 1 is a novel receptor involved in the uptake of myelin by phagocytes. Sci Rep, 2017. 7: p. 44794.

307. Belov, M.E., et al., Design and Performance of a Novel Interface for Combined MatrixAssisted Laser Desorption Ionization at Elevated Pressure and Electrospray Ionization with Orbitrap Mass Spectrometry. Analytical Chemistry, 2017. 89(14): p. 7493-7501.

308. Ellis, S.R., et al., Automated, parallel mass spectrometry imaging and structural identification of lipids. Nat Methods, 2018. 15(7): p. 515-518.

309. Schramm, T., et al., imzML--a common data format for the flexible exchange and processing of mass spectrometry imaging data. J Proteomics, 2012. 75(16): p. 5106-5110.

310. Sud, M., et al., LMSD: LIPID MAPS structure database. Nucleic Acids Res, 2007. 35(Database issue): p. D527-32.

311. Van Nuffel, S., et al., Insights into the MALDI Process after Matrix Deposition by Sublimation Using 3D ToF-SIMS Imaging. Anal Chem, 2018. 90(3): p. 1907-1914.

312. Ruiz-Jimenez, J., et al., Determination of the ubiquinol-10 and ubiquinone-10 (coenzyme Q10) in human serum by liquid chromatography tandem mass spectrometry to evaluate the oxidative stress. J Chromatogr A, 2007. 1175(2): p. 242-8. 
313. Tatsuta, Y., et al., Imaging mass spectrometry analysis of ubiquinol localization in the mouse brain following short-term administration. Sci Rep, 2017. 7(1): p. 12990.

314. Ruiz-Jiménez, J., et al., Determination of the ubiquinol-10 and ubiquinone-10 (coenzyme Q10) in human serum by liquid chromatography tandem mass spectrometry to evaluate the oxidative stress. Journal of Chromatography A, 2007. 1175(2): p. 242-248.

315. Grajchen, E., J.J.A. Hendriks, and J.F.J. Bogie, The physiology of foamy phagocytes in multiple sclerosis. Acta Neuropathol Commun, 2018. 6(1): p. 124.

316. Mailleux, J., et al., Active liver X receptor signaling in phagocytes in multiple sclerosis lesions. Mult Scler, 2018. 24(3): p. 279-289.

317. Bogie, J.F., et al., Myelin alters the inflammatory phenotype of macrophages by activating PPARs. Acta Neuropathol Commun, 2013. 1(1): p. 43.

318. Halmer, R., S. Walter, and K. Fassbender, Sphingolipids: important players in multiple sclerosis. Cell Physiol Biochem, 2014. 34(1): p. 111-8.

319. Marbois, B.N., et al., Analysis of sulfatide from rat cerebellum and multiple sclerosis white matter by negative ion electrospray mass spectrometry. Biochim Biophys Acta, 2000. 1484(1): p. 59-70.

320. Kanter, J.L., et al., Lipid microarrays identify key mediators of autoimmune brain inflammation. Nat Med, 2006. 12(1): p. 138-43.

321. Wheeler, D., et al., A defect of sphingolipid metabolism modifies the properties of normal appearing white matter in multiple sclerosis. Brain, 2008. 131(Pt 11): p. 3092-102.

322. O'Brien, J.S. and G. Rouser, The fatty acid composition of brain sphingolipids: sphingomyelin, ceramide, cerebroside, and cerebroside sulfate. J Lipid Res, 1964. 5(3): p. 339-42.

323. Cantuti-Castelvetri, L., et al., Defective cholesterol clearance limits remyelination in the aged central nervous system. Science, 2018. 359(6376): p. 684-688.

324. Bogie, J.F., et al., Myelin-derived lipids modulate macrophage activity by liver X receptor activation. PLoS One, 2012. 7(9): p. e44998.

325. Dautel, S.E., et al., Lipidomics reveals dramatic lipid compositional changes in the maturing postnatal lung. Sci Rep, 2017. 7: p. 40555.

326. Papamandjaris, A.A., D.E. Macdougall, and P.J.H. Jones, Medium chain fatty acid metabolism and energy expenditure: Obesity treatment implications. Life Sciences, 1998. 62(14): p. 1203-1215.

327. Jove, M., et al., Lipidomics reveals altered biosynthetic pathways of glycerophospholipids and cell signaling as biomarkers of the polycystic ovary syndrome. Oncotarget, 2018. 9(4): p. 4522-4536.

328. Trepanier, M.O., et al., High-resolution lipidomics coupled with rapid fixation reveals novel ischemia-induced signaling in the rat neurolipidome. J Neurochem, 2017. 140(5): p. 766-775.

329. Tyurina, Y.Y., et al., Redox (phospho)lipidomics of signaling in inflammation and programmed cell death. J Leukoc Biol, 2019. 106(1): p. 57-81.

330. Mao, G., et al., Mitochondrial Redox Opto-Lipidomics Reveals Mono-Oxygenated Cardiolipins as Pro-Apoptotic Death Signals. ACS Chem Biol, 2016. 11(2): p. 530-40.

331. Baenke, F., et al., Hooked on fat: the role of lipid synthesis in cancer metabolism and tumour development. Dis Model Mech, 2013. 6(6): p. 1353-63.

332. Gomez de Cedron, M. and A. Ramirez de Molina, Microtargeting cancer metabolism: opening new therapeutic windows based on lipid metabolism. J Lipid Res, 2016. 57(2): p. 193-206.

333. Martinez-Seara, H., et al., Interplay of unsaturated phospholipids and cholesterol in membranes: effect of the double-bond position. Biophys J, 2008. 95(7): p. 3295-305.

334. Shinzawa-Itoh, K., et al., Structures and physiological roles of 13 integral lipids of bovine heart cytochrome c oxidase. EMBO J, 2007. 26(6): p. 1713-25.

335. Renne, M.F. and A. de Kroon, The role of phospholipid molecular species in determining the physical properties of yeast membranes. FEBS Lett, 2018. 592(8): p. 1330-1345. 
336. Ma, X., et al., Identification and quantitation of lipid $C=C$ location isomers: $A$ shotgun lipidomics approach enabled by photochemical reaction. Proceedings of the National Academy of Sciences, 2016. 113(10): p. 2573-2578.

337. Kelley, N.S., N.E. Hubbard, and K.L. Erickson, Conjugated linoleic acid isomers and cancer. J Nutr, 2007. 137(12): p. 2599-607.

338. Khandekar, M.J., P. Cohen, and B.M. Spiegelman, Molecular mechanisms of cancer development in obesity. Nat Rev Cancer, 2011. 11(12): p. 886-95.

339. Beloribi-Djefaflia, S., S. Vasseur, and F. Guillaumond, Lipid metabolic reprogramming in cancer cells. Oncogenesis, 2016. 5: p. e189.

340. Roche, H.M., et al., Isomer-dependent metabolic effects of conjugated linoleic acid: insights from molecular markers sterol regulatory element-binding protein-1c and LXRalpha. Diabetes, 2002. 51(7): p. 2037-44.

341. Ryder, J.W., et al., Isomer-specific antidiabetic properties of conjugated linoleic acid. Improved glucose tolerance, skeletal muscle insulin action, and UCP-2 gene expression. Diabetes, 2001. 50(5): p. 1149-57.

342. Jain, S.K., et al., Erythrocyte membrane lipid peroxidation and glycosylated hemoglobin in diabetes. Diabetes, 1989. 38(12): p. 1539-43.

343. Han, X., et al., Diabetes-induced changes in specific lipid molecular species in rat myocardium. Biochemical Journal, 2000. 352(1): p. 79-89.

344. Stahlman, M., et al., Clinical dyslipidaemia is associated with changes in the lipid composition and inflammatory properties of apolipoprotein-B-containing lipoproteins from women with type 2 diabetes. Diabetologia, 2012. 55(4): p. 1156-66.

345. Wenk, M.R., Lipidomics: new tools and applications. Cell, 2010. 143(6): p. 888-95.

346. Stahlman, M., et al., High-throughput shotgun lipidomics by quadrupole time-of-flight mass spectrometry. J Chromatogr B Analyt Technol Biomed Life Sci, 2009. 877(26): p. 2664-72.

347. Yang, K., et al., Automated lipid identification and quantification by multidimensional mass spectrometry-based shotgun lipidomics. Anal Chem, 2009. 81(11): p. 4356-68.

348. Ejsing, C.S., et al., Global analysis of the yeast lipidome by quantitative shotgun mass spectrometry. Proc Natl Acad Sci U S A, 2009. 106(7): p. 2136-41.

349. Han, X., Characterization and direct quantitation of ceramide molecular species from lipid extracts of biological samples by electrospray ionization tandem mass spectrometry. Anal Biochem, 2002. 302(2): p. 199-212.

350. Natwarlal Vadgama, R., A. Anil, and A. Lali, Identification of Glyceryl MonoRicinoleate (GMR) isomers using RP-HPLC and regio-specificity of lipases. Prep Biochem Biotechnol, 2020. 50(4): p. 401-407.

351. Triebl, A., et al., Lipidomics by ultrahigh performance liquid chromatography-high resolution mass spectrometry and its application to complex biological samples. J Chromatogr B Analyt Technol Biomed Life Sci, 2017. 1053: p. 72-80.

352. Yuan, Z.X., et al., Lipidomic profiling of targeted oxylipins with ultra-performance liquid chromatography-tandem mass spectrometry. Anal Bioanal Chem, 2018. 410(23): p. 60096029.

353. Holcapek, M. and W.C. Byrdwell, Handbook of Advanced Chromatography/Mass Spectrometry Techniques. 2017: Elsevier.

354. Lisa, M. and M. Holcapek, High-Throughput and Comprehensive Lipidomic Analysis Using Ultrahigh-Performance Supercritical Fluid Chromatography-Mass Spectrometry. Anal Chem, 2015. 87(14): p. 7187-95.

355. Rustam, Y.H. and G.E. Reid, Analytical Challenges and Recent Advances in Mass Spectrometry Based Lipidomics. Anal Chem, 2018. 90(1): p. 374-397.

356. Ekroos, K., et al., Charting molecular composition of phosphatidylcholines by fatty acid scanning and ion trap MS3 fragmentation. J Lipid Res, 2003. 44(11): p. 2181-92.

357. Batchu, K.C., et al., Factors regulating the substrate specificity of cytosolic phospholipase A2-alpha in vitro. Biochim Biophys Acta, 2016. 1861(11): p. 1597-1604. 
358. Zhao, X., et al., Lipid Alterations during Zebrafish Embryogenesis Revealed by Dynamic Mass Spectrometry Profiling with $C=C$ Specificity. J Am Soc Mass Spectrom, 2019. 30(12): p. 2646-2654.

359. Su, Y., et al., Mapping Lipid $C=C$ Location Isomers in Organ Tissues by Coupling Photochemical Derivatization and Rapid Extractive Mass Spectrometry. Int J Mass Spectrom, 2019. 445.

360. Arne, J.M., et al., Allosteric modulation of the substrate specificity of acyl-CoA wax alcohol acyltransferase 2. J Lipid Res, 2017. 58(4): p. 719-730.

361. Awad, A.B., et al., 18:1 $n 7$ fatty acids inhibit growth and decrease inositol phosphate release in HT-29 cells compared to $n 9$ fatty acids. Cancer Letters, 1995. 91(1): p. 55-61.

362. de Jonge, H.W., et al., Eicosapentaenoic acid incorporation in membrane phospholipids modulates receptor-mediated phospholipase $C$ and membrane fluidity in rat ventricular myocytes in culture. J Mol Cell Cardiol, 1996. 28(5): p. 1097-108.

363. Schroit, A.J. and R. Gallily, Macrophage fatty acid composition and phagocytosis: effect of unsaturation on cellular phagocytic activity. Immunology, 1979. 36(2): p. 199-205.

364. Zeng, Y., X. Han, and R.W. Gross, Phospholipid subclass specific alterations in the passive ion permeability of membrane bilayers: separation of enthalpic and entropic contributions to transbilayer ion flux. Biochemistry, 1998. 37(8): p. 2346-55.

365. Pham, H.T., et al., Ozone-induced dissociation of conjugated lipids reveals significant reaction rate enhancements and characteristic odd-electron product ions. J Am Soc Mass Spectrom, 2013. 24(2): p. 286-96.

366. Paine, M.R.L., et al., Mass Spectrometry Imaging with Isomeric Resolution Enabled by Ozone-Induced Dissociation. Angew Chem Int Ed Engl, 2018. 57(33): p. 10530-10534.

367. Klein, D.R., et al., Desorption Electrospray Ionization Coupled with Ultraviolet Photodissociation for Characterization of Phospholipid Isomers in Tissue Sections. Anal Chem, 2018. 90(17): p. 10100-10104.

368. Waldchen, F., B. Spengler, and S. Heiles, Reactive Matrix-Assisted Laser Desorption/Ionization Mass Spectrometry Imaging Using an Intrinsically Photoreactive Paterno-Buchi Matrix for Double-Bond Localization in Isomeric Phospholipids. J Am Chem Soc, 2019. 141(30): p. 11816-11820.

369. Bednarik, A., et al., An On-Tissue Paterno-Buchi Reaction for Localization of CarbonCarbon Double Bonds in Phospholipids and Glycolipids by Matrix-Assisted LaserDesorption-Ionization Mass-Spectrometry Imaging. Angew Chem Int Ed Engl, 2018. 57(37): p. 12092-12096.

370. Kuo, T.H., et al., Deep Lipidomics and Molecular Imaging of Unsaturated Lipid Isomers: A Universal Strategy Initiated by mCPBA Epoxidation. Anal Chem, 2019. 91(18): p. 11905-11915.

371. Feng, Y., et al., Identification of Double Bond Position Isomers in Unsaturated Lipids by m-CPBA Epoxidation and Mass Spectrometry Fragmentation. Anal Chem, 2019. 91(3): p. 1791-1795.

372. Zhao, Y., et al., Identification and Quantitation of C horizontal lineC Location Isomers of Unsaturated Fatty Acids by Epoxidation Reaction and Tandem Mass Spectrometry. Anal Chem, 2017. 89(19): p. 10270-10278.

373. Tang, S., H. Cheng, and X. Yan, On-Demand Electrochemical Epoxidation in NanoElectrospray Ionization Mass Spectrometry to Locate Carbon-Carbon Double Bonds. Angew Chem Int Ed Engl, 2020. 59(1): p. 209-214.

374. Deng, J., et al., Coupling Paterno-Buchi Reaction with Surface-Coated Probe Nanoelectrospray Ionization Mass Spectrometry for In Vivo and Microscale Profiling of Lipid C horizontal lineC Location Isomers in Complex Biological Tissues. Anal Chem, 2019. 91(7): p. 4592-4599.

375. Feider, C.L., et al., Double Bond Characterization of Free Fatty Acids Directly from Biological Tissues by Ultraviolet Photodissociation. Anal Chem, 2020. 92(12): p. 83868395 . 
376. Macias, L.A., et al., Hybrid $193 \mathrm{~nm}$ Ultraviolet Photodissociation Mass Spectrometry Localizes Cardiolipin Unsaturations. Anal Chem, 2019. 91(19): p. 12509-12516.

377. Claes, B.S.R., et al., Imaging Isomers on a Biological Surface: A Review. Mass Spectrometry, 2019. 8(1): p. A0078-A0078.

378. $\mathrm{Vu}, \mathrm{N}$., et al., Ozone-induced dissociation on a traveling wave high-resolution mass spectrometer for determination of double-bond position in lipids. Rapid Communications in Mass Spectrometry, 2017. 31(17): p. 1415-1423.

379. Poad, B.L.J., et al., High-Pressure Ozone-Induced Dissociation for Lipid Structure Elucidation on Fast Chromatographic Timescales. Analytical Chemistry, 2017. 89(7): p. 4223-4229.

380. Poad, B.L.J., et al., Online Ozonolysis Combined with Ion Mobility-Mass Spectrometry Provides a New Platform for Lipid Isomer Analyses. Anal Chem, 2018. 90(2): p. 12921300.

381. Vu, N., et al., Ozone-induced dissociation on a traveling wave high-resolution mass spectrometer for determination of double-bond position in lipids. Rapid Commun Mass Spectrom, 2017. 31(17): p. 1415-1423.

382. Barré, F., et al., Faster raster matrix-assisted laser desorption/ionization mass spectrometry imaging of lipids at high lateral resolution. International Journal of Mass Spectrometry, 2019. 437: p. 38-48.

383. Strohalm, M., et al., mMass data miner: an open source alternative for mass spectrometric data analysis. Rapid Commun Mass Spectrom, 2008. 22(6): p. 905-8.

384. Liebisch, G., et al., Shorthand notation for lipid structures derived from mass spectrometry. J Lipid Res, 2013. 54(6): p. 1523-30.

385. Young, R.S.E., et al., Apocryphal FADS2 activity promotes fatty acid diversification in cancer. Cell Reports, 2021. 34(6): p. 108738.

386. Tang, F., et al., Rapid In Situ Profiling of Lipid C=C Location Isomers in Tissue Using Ambient Mass Spectrometry with Photochemical Reactions. Analytical Chemistry, 2018. 90(9): p. 5612-5619.

387. Batarseh, A.M., et al., Discrimination of isobaric and isomeric lipids in complex mixtures by combining ultra-high pressure liquid chromatography with collision and ozoneinduced dissociation. International Journal of Mass Spectrometry, 2018. 431: p. 27-36.

388. Bruce, K.D., A. Zsombok, and R.H. Eckel, Lipid Processing in the Brain: A Key Regulator of Systemic Metabolism. Front Endocrinol (Lausanne), 2017. 8: p. 60.

389. Pradas, I., et al., Lipidomics Reveals a Tissue-Specific Fingerprint. Front Physiol, 2018. 9: p. 1165.

390. Fahy, E., et al., Lipid classification, structures and tools. Biochim Biophys Acta, 2011. 1811(11): p. 637-47.

391. Kuo, T.C. and Y.J. Tseng, LipidPedia: a comprehensive lipid knowledgebase. Bioinformatics, 2018. 34(17): p. 2982-2987.

392. Fahy, E., et al., A comprehensive classification system for lipids. J Lipid Res, 2005. 46(5): p. 839-61.

393. Campbell, J.L. and T. Baba, Near-Complete Structural Characterization of Phosphatidylcholines Using Electron Impact Excitation of Ions from Organics. Analytical Chemistry, 2015. 87(11): p. 5837-5845.

394. Baba, T., et al., Quantitative structural multiclass lipidomics using differential mobility: electron impact excitation of ions from organics (EIEIO) mass spectrometry. J Lipid Res, 2018. 59(5): p. 910-919.

395. Choi, J., et al., Comprehensive analysis of phospholipids in the brain, heart, kidney, and liver: brain phospholipids are least enriched with polyunsaturated fatty acids. Mol Cell Biochem, 2018. 442(1-2): p. 187-201.

396. Liebisch, G., et al., Update on LIPID MAPS Classification, Nomenclature and Shorthand Notation for MS-derived Lipid Structures. J Lipid Res, 2020. 
Chapter 12 APPENDIX 


\section{FUNDING AND CONFLICTS OF INTEREST}

\section{CHAPTER 2}

This work has been made possible with the financial support of the Dutch province of Limburg through the LINK program. The authors also acknowledge funding from Interreg VEMR and the Netherlands Ministry of Economic Affairs within the "EURLIPIDS" project.

\section{CHAPTER 3}

A portion of this work was performed at the National High Magnetic Field Laboratory ICR User Facility, which is supported by the National Science Foundation Division of Chemistry through DMR-1644779 and the State of Florida. Part of this work was financially supported through the LINK program of the Dutch province of Limburg. Part of this work was financially supported through the EURLIPIDS program of Euregio.

\section{CHAPTER 4}

This work has been made possible with the financial support of the Dutch province of Limburg through the LINK program. A.P.B, R.M.A.H and S.R.E are grateful for funding from Interreg V EMR and the Netherlands Ministry of Economic Affairs within the "EURLIPIDS" project (EMR23) and by the Flemish Fund for Scientific Research (FWO Vlaanderen) and special research fund UHasselt (BOF). We are grateful to both Dr. Carine Peutz-Kootstra and Dr. Marion Gijbels (Maastricht University Medical Centre) for help with kidney histology and both Dr. Sara Tortorella (Molecular Horizon Srl, Italy) and Paolo Tiberi (Molecular Discovery Ltd, UK) for help and support with LipostarMSI software.

\section{CONFLICTS OF INTEREST}

Mikhail Belov is the general manager of Spectroglyph LLC, the supplier of the dualfunnel MALDI/ESI Injector ${ }^{\mathrm{TM}}$ interface. The remaining authors declare no conflict of interest.

\section{CHAPTER 5}

Part of this work was supported by the Dutch province of Limburg through the LINK program. B.S.R.C. and R.M.A.H. acknowledge funding from the National Cancer Institute of the NIH, Grant No. ROI1 CA213492. A.P.B, S.R.E., and R.M.A.H. acknowledge funding from Interreg V-A EMR and the Netherlands Ministry of Economic Affairs within the "Interreg Euro-Maas-Rijn" project (project number EMR23). B.L.J.P. and S.J.B. acknowledge funding from the Australian Research Council (LP180100238 and DP190101486). The authors are grateful to Tiffany Porta-Siegel and Hang Nguyen for manuscript editing. 


\section{Chapter 13 List OF PUBliCATIONS}




\section{This thesis is based on the following publications.}

Bowman, Andrew P., Ron M. A. Heeren, et al. "Advances in Mass Spectrometry Imaging Enabling Observation of Localised Lipid Biochemistry within Tissues." TrAC Trends in Analytical Chemistry, vol. 120, 2019, p. 115197, doi:10.1016/j.trac.2018.07.012.

Bowman, Andrew P., Jeroen F. J. Bogie, et al. "Evaluation of Lipid Coverage and High Spatial Resolution MALDI-Imaging Capabilities of Oversampling Combined with Laser Post-Ionisation." Analytical and Bioanalytical Chemistry, vol. 412, no. 10, 2019, pp. 2277-89. Crossref, doi:10.1007/s00216-019-02290-3.

Bowman, Andrew P., Greg T. Blakney, et al. "Ultra-High Mass Resolving Power, Mass Accuracy, and Dynamic Range MALDI Mass Spectrometry Imaging by 21-T FT-ICR MS.” Analytical Chemistry, vol. 92, no. 4, 2020, pp. 3133-42. Crossref, doi:10.1021/acs.analchem.9b04768.

Claes, Britt S. R., Bowman, Andrew P., Poad, Berwyck L.J., Heeren, Ron M.A., Blanksby, Stephen J., Ellis, Shane R. "High Throughput and High Sensitivity Isomer-Resolved Imaging of Lipids by Integration of Ozone-Induced Dissociation With a MALDI-QTOF Mass Spectrometer." Analytical Chemistry, 2021, Crossref, doi:10.1021/acs.analchem.1c01377.

Bowman, Andrew P., Claes, Britt S. R., Poad, Berwyck L.J., Heeren, Ron M.A., Blanksby, Stephen J., Ellis, Shane R. "Negative Mode Imaging of Phospholipids Using Ozone-Induced Dissociation.” In Preparation. 


\section{Other Publications}

Bogie JFJ, Grajchen E, Wouters E, Corrales AG, Dierckx T, Vanherle S, Mailleux J, Gervois P, Wolfs E, Dehairs J, Van Broeckhoven J, Bowman AP, Lambrichts I, Gustafsson JÅ, Remaley AT, Mulder M, Swinnen JV, Haidar M, Ellis SR, Ntambi JM, Zelcer N, Hendriks JJA. "Stearoyl-CoA Desaturase-1 Impairs the Reparative Properties of Macrophages and Microglia in the Brain." Journal of Experimental Medicine, vol. 217, no. 5, 2020. Crossref, doi:10.1084/jem.20191660.

Capolupo L, Khven I, Mazzeo L, Glousker G, Russo F, Montoya JP, Ho S, Bhandari DR, Bowman AP, Ellis SR, Guiet R, Muthing J, Spengler B, Heeren RM, Dotto GP, La Manno G, D'Angelo G. "Sphingolipid Control of Fibroblast Heterogeneity Revealed by Single-Cell Lipidomics.” BioRxiv, 2021. Crossref, doi:10.1101/2021.02.23.432420.

Dudek, M; Pfister, D; Donakonda, S; Filpe, P; Schneider, A; Laschinger, M; Hartmann, D; Hüser, N; Meiser, P; Bayerl, F; Inverso, D; Wigger, J; Sebode, M; Öllinger, R; Rad, R; Hegenbarth, S; Anton, M; Guillot, A; Bowman, AP; Heide, D; Müller, F; Ramadori, P; Leone, V; Garcia-Caceres, C; Gruber, T; Seifert, G; Kabat, AM; Mallm, JP; Reider, S; Effenberger, M; Roth, S; Billeter, AT; MüllerStich, B; Pearce, EJ; Koch-Nolte, F; Käser, R; Tilg, H; Thimme, R; Boettler, T; Tacke, F; Dufour, JF; Haller, D; Murray, PJ; Heeren, RMA; Zehn, D; Böttcher, JP; Heikenwälder, M; Knolle, PA. "Auto-aggressive CXCR6+ CD8 T cells cause liver immune pathology in NASH." Nature, vol. 592, no. 7854, 2021. Crossref, doi:10.1038/s41586-021-03233-8

Kruining, D van; Luo, Q; Echten-Deckert, G van; Mielke, MM; Bowman, AP; Ellis, SRE; Oliveira, TG; Martinez-Martinez, P. "Sphingolipids as Prognostic Biomarkers of Neurodegeneration, Neuroinflammation, and Psychiatric Diseases and Their Emerging Role in Lipidomic Investigation Methods." Advanced Drug Delivery Reviews, vol. 159, 2020, pp. 232-44. Crossref, doi:10.1016/j.addr.2020.04.009. 
Tortorella, S.; Tiberi, P; Bowman, AP; Claes, BSR; Ščupáková, K; Heeren, RMA; Ellis, SRE; Cruciani, G. "LipostarMSI: Comprehensive, Vendor-Neutral Software for Visualization, Data Analysis, and Automated Molecular Identification in Mass Spectrometry Imaging." Journal of the American Society for Mass Spectrometry, vol. 31, no. 1, 2019, pp. 155-63. Crossref, doi:10.1021/jasms.9b00034.

Vos, DRN; Bowman, AP; Heeren, RMA; Balluff, B; Ellis, SRE. "Class-Specific Depletion of Lipid Ion Signals in Tissues upon Formalin Fixation." International Journal of Mass Spectrometry, vol. 446, 2019, p. 116212. Crossref, doi:10.1016/j.ijms.2019.116212.

Young, RSE; Bowman, AP; Williams, ED; Tousignant, KD; Bidgood, CL; Narreddula, VR; Gupta, R; Marshall, DL; Poad, BLJ; Nelson, CC; Ellis, SR; Heeren, RMA; Sadowski, MC; Blanksby, SJ. "Apocryphal FADS2 Activity Promotes Fatty Acid Diversification in Cancer." Cell Reports, vol. 34, no. 6, 2021, p. 108738. Crossref, doi:10.1016/j.celrep.2021.108738.

Young, RSE; Bowman, AP; Williams, ED; Shepherd, B; Perren, A; Poad, BLJ; Ellis, SRE; Heeren, RMA; Sadowski, MC; Blanksby, SJ. "Isomer-Resolved Imaging of Prostate Cancer Tissues Reveals Specific Lipid Unsaturation Profiles Associated with Lymphocytes and Abnormal Prostate Epithelia." Frontiers Endocrinology, 2021, Submitted.

Young, RSE; Bowman, AP; Tousignant, KD; Poad, BLJ; Eijkel, GB; Nelson, CC; Ellis, SRE; Heeren, RMA; Sadowski, MC; Blanksby, SJ. "Phospholipid snisomers reveal discrete de novo and extracellular fatty acid metabolism." In preparation. 
Chapter 14 ACKNOWLEDGEMENTS 
Never before have I undertaken such a task as writing this thesis. When I started my $\mathrm{PhD} 4$ years ago, the idea of summing up years of work into a single book seemed impossible, and I'm still not sure it isn't. I don't really consider myself much of a speech writer, but, that aside, there are some people without whom this book would not be here now.

Melissa, you helped support me through 4 years of stress and insanity. Without you, I probably wouldn't have been able to manage myself, or to dedicate nearly as much time to the selfish pursuit of a PhD. Nor would I have had something to look forward to when I came home (Zazou not withstanding).

Britt, you have been a bastion of grunt work and help in producing at least $40 \%$ of this thesis. If it weren't for you acting as my sometimes colleague sometimes lackey, I don't think this thesis would be nearly as complete as it is.

Bryn, while we never really worked together in the lab, you were definitely one of the most fun people to just hang out with during my time at M4I. We'll have to get back together one of these days and finish all of those Bond movies.

Joel, a prime example of never giving up, even if none of the experiments work the way you want. And a shining beacon to tell the world that there's always a way forward, even if its not the direction you expect.

Ben, you joined the lab fairly late into my $\mathrm{PhD}$, but you and I work pretty well together. I'm sorry that I've left you with a couple of misbehaving instruments, but I'm sure you'll figure them out.

Darya, I appreciate so much that you bit the bullet for the rest of us by having far too many interns simultaneously. And despite that, you've still managed to be in the lab, doing your own work. There's something inspirational in that.

Daan, from meeting you at the very beginning of my $\mathrm{PhD}$ and extending until years after you left, you've been a friend to me. Thank you for indulging me in playing Pathfinder and tabletop games alike for the whole length of my $\mathrm{PhD}$.

Ian, its been fun watching you transition rapidly from a lowly postdoc into an associate professor, while learning how to do MSI and operate entirely new (and still semi-secret) instruments. I wish you the best of luck (knowing new instruments, you'll need it).

Ron, after meeting you and Shane at the IMSC in Toronto all those years ago, you've never once stopped believing in me. From experimental support to being a sounding board when I thought I wasn't good enough, you're everything that I could ever hope that a PI would be. 
Shane, I've never before met somebody so dedicated to the idea of science. Your drive to uncover the next thing, even when that took you from M4I back down to Australia, has been amazing to watch. Thank you for inspiring met to dig a little bit deeper and look a little bit further.

Fred, we never really got to work together much, but you let me steal all of your notes to give a presentation, so I can't say anything bad about you.

Philippe, thanks to you, I could focus on my $\mathrm{PhD}$ without having to worry about so much of the bureaucracy of the Eurlipids. My apologies for not reporting nearly as much as I should.

Tiff, thanks to you I got to explore more of the Netherlands by foot than I ever thought I would. I'm still upset that COVID cut short the chance for you, Ben, Bryn, and I to go hiking more.

$\mathbf{K y}$, you've been a great friend, and a welcome person to vent to in times when it felt like I was getting nothing right.

Roshi, if there's one person on this planet who I could find better inspiration in to get shit done, I've never met them.

To everyone else at M4I, Charles, Benjamin, Bo, Jian-Hua, Berta, Eva, Gert, Ana, Frans, Andrej, Mirella, Peiliang, Laura, Maarten, Aljoscha, Kasper, other Kasper, Anjusha, Lucia, Sylvia, Sebastiaan, Roel, Isabeau, Yuandi, thank you for making my stay here in the Netherlands such an interesting one, whether we talked only once or you just needed me to beat an instrument in line. To the people I've collaborated with, Jeroen, Jerome, Reuben, Bez, Stephen, Wojciech, Percy, Michael, thank you for choosing me to work with, and I'm glad for all that I could do to help you guys achieve your dreams as much as you helped me achieve mine.

My family, without whom I would not be here. The lot of you nearly drove me crazy, but I suppose that that's really what made me capable of going after a $\mathrm{PhD}$. So, thanks for all of it. 


\section{Chapter 15 CURRICULUM VITAE}

Andrew Bowman was born in Wichita, Kansas in the United States late in 1990. After graduating high school in the same town he was born in, Andrew fled to Cornell College to pursue a Bachelor's Degree in Arts, majoring in biology. In 2012 he graduated with said degree and spent a fruitless pair of years not being hired in his field. Beginning in January of 2015, he joined the lab of Alexandre Shvartsburg at Wichita State University. He studied Field-Asymmetric Waveform Ion Mobility Spectrometry, focusing on the separation of lipid isomers. In

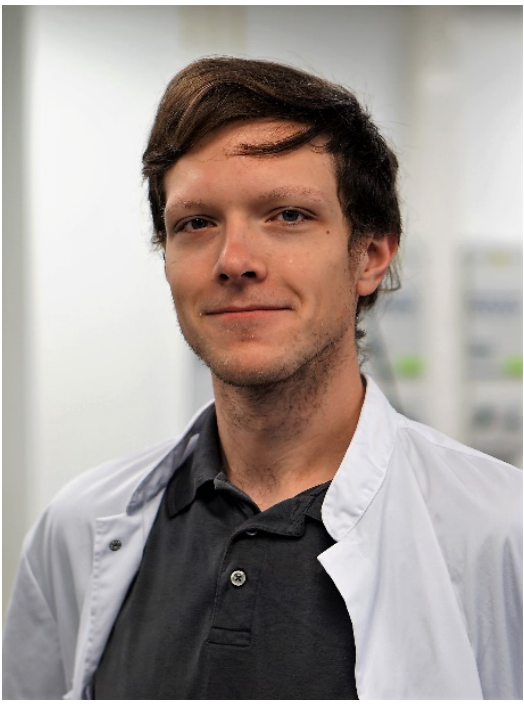
2018 he received a Master of Science in Analytical Chemistry from Wichita State University (Wichita, Kansas), merely 9 months after beginning his $\mathrm{PhD}$ work in the Maastricht MultiModal Molecular Imaging Institute (Maastricht, The Netherlands).

In 2017, Andrew joined the lab of Ron Heeren under the auspices of the Eurlipids Consortium, being directed by Shane Ellis. As an early $\mathrm{PhD}$ candidate, Andrew performed a month-long stint at the National High Magnetic Field Laboratory (Tallahassee, Florida), learning how to operate FT-ICR instruments. Upon his return to Europe, he attended the Mass Spectrometry School in Biotechnology and Medicine, before finally deigning to return to the Netherlands and begin his doctoral work. During his $\mathrm{PhD}$ work, Andrew collaborated with numerous institutes and companies, producing 5 first-author papers, 9 collaborative papers, and participating in an invited talk for Bruker Daltonik.

Outside of his $\mathrm{PhD}$ work, Andrew is an avid tabletop gamer, game mastering for all 4 years of his $\mathrm{PhD}$ both online and in person. He also enjoys hiking, reading, and offering unsolicited advice to friends and passersby.

He will be transferring to the pharmaceutical world as a Postdoctoral Fellow with AbbVie. 